\title{
Lucas Diego Rodrigues Ferreira
}

\section{DEVELOPMENT OF A ROTARY THERMOMAGNETIC MOTOR FOR THERMAL ENERGY CONVERSION.}

\section{São Paulo}

2019 


\section{Lucas Diego Rodrigues Ferreira}

\section{DEVELOPMENT OF A ROTARY THERMOMAGNETIC MOTOR FOR THERMAL ENERGY CONVERSION.}

Doctor's thesis presented to Escola

Politécnica da Universidade de São Paulo is fulfillment of the requirements for the Doctor of Science degree.

São Paulo

2019 


\section{Lucas Diego Rodrigues Ferreira}

\section{DEVELOPMENT OF A ROTARY THERMOMAGNETIC MOTOR FOR THERMAL ENERGY CONVERSION.}

Doctor's thesis presented to Escola Politécnica da Universidade de São Paulo is fulfillment of the requirements for the Doctor of Science degree.

Area: Mechanical Engineering Advisor: Prof. Ph.D. Oswaldo Horikawa

\section{São Paulo}


Autorizo a reprodução e divulgação total ou parcial deste trabalho, por qualquer meio convencional ou eletrônico, para fins de estudo e pesquisa, desde que citada a fonte.

Este exemplar foi revisado e corrigido em relação à versão original, sob responsabilidade única do autor e com a anuência de seu orientador.

São Paulo, de de

Assinatura do autor:

Assinatura do orientador:

\section{Catalogação-na-publicação}

Ferreira, Lucas Diego Rodrigues

DEVELOPMENT OF A ROTARY THERMOMAGNETIC MOTOR FOR

THERMAL ENERGY CONVERSION / L. D. R. Ferreira -- versão corr. -- São

Paulo, 2019.

$173 \mathrm{p}$.

Tese (Doutorado) - Escola Politécnica da Universidade de São Paulo. Departamento de Engenharia Mecânica.

1.Materiais Magnéticos 2.Simulação de Sistemas 3.Motores Térmicos 4.Energia Térmica I.Universidade de São Paulo. Escola Politécnica.

Departamento de Engenharia Mecânica II.t. 
To my loving and supporting parents Luiz Fernando and Roseli Aparecida, And to my partner in love and life, Juliana Rodrigues 


\section{ACKNOWLEDGMENTS}

This thesis was made possible thanks to the contributions of many people. In first place I would like to thank my advisor, Prof. Oswaldo Horikawa, he was always willing to help me in a kind manner in every step of this Ph.D. Following my gratitude is directed to Prof. Sergio Gama, who exercised a mentorship advisory position through the process of research, with many insightful and enlightening discussions.

I am also extremely grateful to my colleagues both in the University of São Paulo, and the L3M laboratory from the Federal University of São Paulo, especial regards are destined to Carlos V.X. Bessa, Rogério Issamu Yamamoto, André Paganotti, Prof. Ricardo Galdino, the technicians Cesar Celestino de Souza e Silva and Samuel de Paula Rodrigues, and the many other students and collaborators who have made the research a much easier and enjoyable process.

A special regard is designated in memoriam of Professor Isaias da Silva, his base research is the fundament of the Rotary Thermomagnetic Motor developed in this thesis, but more importantly, he will always be missed as a colleague and a friend.

Finally, I would like to thank my parents and family, without whom this research would be impossible, especially to my partner Juliana Rodrigues. 


\section{SUMMARY}

Thermomagnetic motors can represent an alternative for the conversion of heat into mechanical energy, limited by the critical transition temperature $\left(T_{C}\right)$ of the used magnetic materials. Thus, by using materials with a $T_{C}$ close to room temperature, the energy available in the form of low-grade heat sources can be converted into useful mechanical work. This thesis proposes the development of a thermomagnetic motor to be operated with heat sources at temperatures in the range from 343 to $353 \mathrm{~K}$, and a heat sink at room temperature, using water as the heat transfer fluid, presenting a novel approach to the construction of thermomagnetic devices. The design of this thermomagnetic motor was developed with the intent of producing a rotary movement, working similarly to an electric stepper motor, where instead of the electromagnetic coils being activated by an electric current, plates of a magnetic material change their magnetization state, due to a change in their temperature caused by the heat transfer with the heat transfer fluid.

The analysis of the thermomagnetic motor proposed was done with the adoption of an integrated approach of numerical simulation and experimental validation. The evaluation of the motor is divided into the three main physical phenomena it encompasses: the magnetic field source, the heat transfer processes involved in the change of temperature of the magnetic material, and the system dynamics and power production. Each of these systems was modeled using computational tools. These models were then validated according to the data measured, obtained from a test stand of an idealized thermomagnetic motor, and for a rotary thermomagnetic motor. This methodology allowed a more comprehensive understanding of the critical working principles of the motor developed, and with that a fast advancement of the technology through a validated computational model.

The computational models helped to identify the critical components to be improved in

the development of these motors. These parameters can be guidelines for the design of thermomagnetic motors. One of the ways identified to produce a significant performance improvement, in the simulations, was the adoption of a control strategy that promotes the regeneration of heat in the plates of magnetic material, through which an improvement in the efficiency of 2.7 times could be achieved.

Keywords: Magnetic Materials, Systems Simulations, Heat Engine, Thermal Energy 


\section{RESUMO}

Motores termomagnéticos representam uma alternativa para a conversão de calor em energia mecânica, limitada apenas pela temperatura crítica da transição termomagnética $\left(T_{C}\right)$ dos materiais magnéticos. Ao usar materiais com $T_{C}$ próximo à temperatura ambiente, pode-se realizar a conversão da energia contida nas chamadas fontes pobres de calor, produzindo trabalho mecânico útil. Esta tese propõe o desenvolvimento de um motor termomagnético para operação com fontes de calor com temperaturas entre 343 e $353 \mathrm{~K}$, e resfriamento à temperatura ambiente, utilizando a água como fluído de troca térmica, apresentando uma abordagem inovadora para dispositivos termomagnéticos. O motor foi projetado para produção de movimento rotativo de um eixo, per meio de um princípio similar ao de um motor de passo, no qual em vez de bobinas ativadas pela passagem de corrente elétrica, placas de material magnético sofrem uma mudança em seu estado de magnetização, devido à mudança de temperatura, causada pela troca de calor com a água.

A análise do motor termomagnético proposto foi realizada com a adoção de uma abordagem integrada de simulações numéricas e validação experimental, dividindo a avaliação dos motores nos três principais fenômenos físicos envolvidos em seu funcionamento: a fonte de campo magnético, o processo de troca térmica envolvido na mudança de temperatura do material magnético, a dinâmica do sistema e produção de potência. Cada um destes sistemas foi modelado usando ferramentas computacionais. Os resultados obtidos foram então validados utilizando dados experimentais, obtidos a partir da construção e caracterização de uma bancada de testes para um motor termomagnético idealizado, e também para o motor termomagnético rotativo construído. Esta metodologia propiciou maior entendimento das funções críticas do motor desenvolvido, e possibilitou ainda sua otimização, através do estudo dos modelos computacionais validados.

Os parâmetros obtidos ajudaram a identificar componentes críticos para melhoria no projeto do motor rotativo construído, e servem também como guias gerais para projetos de motores termomagnéticos. Um dos componentes com elevado potencial de melhoria foi a adoção de uma estratégia de controle para a regeneração do calor nas placas de material magnético, o que possibilitou, nas simulações, uma melhoria até 2,7 vezes na eficiência.

Palavras-chave: Materiais Magnéticos, Simulação de Sistemas, Motores Térmicos. 


\section{LIST OF FIGURES}

Figure 1: The "curious thermo-magnetic motor". 11

Figure 2: Cover of the magazine Scientific American from August 1887, presenting the Pyromagnetic motors developed by Edison. 13

Figure 3: Edison's patents submitted in 1887, (a) the Pyromagnetic Motor, converting heat into mechanical movement, and (b) the Pyromagnetic Dynamo, used to convert heat into electricity directly. 14

Figure 4: Thermo-magnetic motor patented by Tesla, (a) initial position of the motor, and (b) the second stage of the movement of the motor. 16

Figure 5: A Thermo-magnetic motor with two ferromagnetic materials linked and without the action of a spring. 16

Figure 6: Historical development of the research of Thermomagnetic devices for energy conversion, in the graph each bar plot represent a 5 years period.

Figure 7: Network analysis mapping of the systematic bibliometric search of the paper related to the field of thermomagnetic energy conversion within the Web of Science database.

Figure 8: Two different theoretical models used to investigate the basic principles and formulate the efficiency of thermomagnetic devices, in (a) a "ferromagnetic heat engine" proposed by Brailsford (1964), and (b) an "idealized thermomagnetic cycle," proposed by Hsu et al. (2011). 23

Figure 9: The thermomagnetic generator with no movement of the magnetic materials. 24 Figure 10: The levitating thermo-magnetic wheel built by Palmy (2007), in (a) the design of the system, with two lifting magnets (B), Bismuth discs ( $\mathrm{Bi})$, the floating magnet $(\mathrm{Fm})$ and a radiation beam heating Fm. The built system is presented in (b). 26

Figure 11: The thermomagnetic motor built and analyzed by Murakami and Nemoto (1972).... 27 Figure 12: The model of the thermomagnetic motor (a) using a disk of magnetic material to produce work, and a picture of the built concept demonstrator (b) of the thermomagnetic motor using a cylinder of magnetic material. 28 Figure 13: The thermomagnetic device with small dimensions focused on low power energy harvesting developed by Ujihara, Carman and Lee (2007), in (a) the actual device built with dimensions of $2 \cdot 10^{-2} \mathrm{~m} \times 2 \cdot 10^{-2} \mathrm{~m} \mathrm{x} 6 \cdot 10^{-3} \mathrm{~m}$, and in (b) the cross-section of the device during the cooling (higher image) and heating (lower image). 29 
Figure 14: A thermomagnetic-electric generator is operating through the phase transition occurring in a magnetic material during periodic cooling and heating, in (a) a schematic representation of the device, and in (b) a picture of the built device. 30

Figure 15: The miniature magnetic-piezoelectric thermal energy harvester studied by Chen et al. (2015), with the modeling (a) and an experimental device built (b).

Figure 16: The static Thermomagnetic generator studied by Christiaanse and Brück (2014), in (a) the design model, with the red cylinder indicating the heated magnetic material and the blue cylinder the cooled material, in (b) the magnetic material regenerators built, with the power producing coils.

Figure 17: The modeling of the interaction between a magnetic material and an applied magnetic field gradient. 34

Figure 18: The correlation between the magnetic energy (red hatched area) and the magnetic coenergy (black hatched area) densities for a defined $B-H$ curve of a non-linear magnetic material.

Figure 19: Design of the Edison type of Thermomagnetic Motor, in (a) a disk of magnetic material is used to produce a torque, and in (b) a simplified view of the design, focused on the work producing region.

Figure 20: The state of the magnetic material in an Edison type of Thermomagnetic Motor, based on the simplified view presented in Figure 19.b. 38

Figure 21: Simplified design of a Tesla type thermomagnetic motor. 39 Figure 22: Magnetization of Gadolinium for a given applied magnetic field at different temperatures.

Figure 23: The magnetic entropy change (T-s diagram) of Gadolinium.

Figure 24: The cycle developed by the modeled Edison type thermomagnetic motor in the $M(H, T)$ diagram for Gadolinium. 42

Figure 25: The undefined thermodynamic developed by the modeled Edison type TMM in the $\mathrm{T}(\mathrm{s}, \mathrm{H})$ diagram of Gadolinium. 43

Figure 26: From the defined thermodynamic states $\mathrm{A}$ and $\mathrm{C}$, two possible behaviors can be described by a Tesla type TMM, an isothermal (dotted lines) or an adiabatic (solid lines) magnetization/demagnetization. 
Figure 27: The cycle developed by the Tesla type thermomagnetic motor in the $M(H, T)$ diagram for Gadolinium, assuming an adiabatic magnetization/demagnetization process, with the effect of the magnetocaloric effect

Figure 28: The thermodynamic cycle developed by the Tesla type TMM in the $T(s, H)$ diagram of Gadolinium, the red encircled area defines a magnetic equivalent Brayton cycle. 46

Figure 29: A schematic view of the proposed test stand to measure the magnetization work of magnetic materials (a), with a section view of the sample holder (b).

Figure 30: Pictures of the test stand to measure the magnetic force of a magnets assembly over a magnetic material with controlled temperature. 52

Figure 31: The use of a slide-crank mechanism to convert the reciprocating movement of a TMM into a rotary movement, with the flywheel also acting to remove the material from the heat source. 53

Figure 32: The working diagram of the "Step-by-step Thermo-magnetic motor," working in the same principle as the Tesla type TMM. 53

Figure 33: Working scheme of the rotary thermomagnetic motor proposed by Coelho, Gama and Silva (2012), with a step rotation of $60^{\circ}$ between each stage and three magnetic material plates. 54 Figure 34: Mechanical design of the rotary type thermomagnetic motor. 55 Figure 35: Modeling of the rotary Tesla type of thermomagnetic motor developed, in (a) a view of the system assembly and in (b) an exploded view showing the magnets assembly and the way the magnetic plates are fixed to the stator. 56

Figure 36: Magnetization of the intermetallic compound $\mathrm{Gd}_{4.7} \mathrm{Nd}_{0.3} \mathrm{Si}_{4}$ for a given applied magnetic field at different temperatures. 58 Figure 37: The Rotary Thermomagnetic Motor built to demonstrate and validate the technology of TMM for the conversion of low-grade energy sources into useful mechanical energy..... 59 Figure 38: "Horseshoe" type of permanent magnets assembly designed for the test bench of an idealized TMM, dimensions shown in $\mathrm{mm}$.

Figure 39: The design used in the 2D magnetostatic simulation (a) to determine the adequate distance between the magnets $\left(V_{a r}\right.$, in $\left.\mathrm{mm}\right)$, and the resulting applied magnetic field for this varying parameter (b).

Figure 40: Induced magnetic field $(B)$ by the "horseshoe" type of magnets assembly (a), and vector field lines (b), according to initial 2D magnetostatic simulation. 64 
Figure 41: Vector induced field lines of two opposing NdFeB permanent magnets with dimensions of 20x20x100 mm (a), and of a single magnet with the same dimensions (b)......... 66 Figure 42: Applied magnetic field strength as a function of the displacement of the reference permanent magnets assembly (Figure 41.a), used to determine the calibration curve for the Hall sensing element. 67

Figure 43: Calibration curve of the Hall effect sensing element CY-SJ-362A, from ChenYang, with the measured Hall sensor voltage and corresponding applied magnetic field strength......... 68 Figure 44: The dependency of the standard uncertainty on the applied magnetic field for the sensor used to measure the magnetic field.

Figure 45: The experiment to measure the applied magnetic field produced by the "horseshoe" permanent magnets assembly used in the idealized TMM. 70

Figure 46: The measured applied magnetic field strengths as a function of the displacement of the "horseshoe" permanent magnets assembly, with the calculated uncertainties for each measured point. 70

Figure 47: Model of the "horseshoe" type of magnets assembly used in the 3D magnetostatic simulation.

Figure 48: The magnitude of the applied magnetic field produced by the "horseshoe" type of permanent magnets assembly used in the idealized TMM. 72

Figure 49: The permanent magnets assembly used in the rotary TMM, with the orientation of the magnets forming an annular region where the magnetic field is concentrated (a), and the model of the assembly used in the 3D magnetostatic simulation (b).

Figure 50: The results from the 3D magnetostatic simulation, in (a) the induced magnetic field (B) produced by the assembly, as the view from a section plane of the model, in (b) the magnetic force induced in each permanent magnet from positioning in the assembly, values shown in Newtons. 75

Figure 51: Picture of the built magnets assembly, with a protective paint to avoid corrosion of the $\mathrm{NdFeB}$ permanent magnets. 76

Figure 52: The applied magnetic field $\left(\mu_{0} H-\mathrm{mT}\right)$ produced by the magnets assembly, with the field mapping based on the experimental measurement, for a distance of $12.5 \mathrm{~mm}$ from magnets faces. 77 
Figure 53: The magnitude of the applied magnetic field produced by the permanent magnets assembly used in the Rotary TMM, for a line at the center of the annular gap region, as indicated in the inset in the figure, and at the center of the assembly.... 78 Figure 54: Specific heat capacity as a function of the temperature for Gadolinium, for applied magnetic field strengths from 0 to $1.4 \mathrm{~T}$.

Figure 55: Specific heat capacity as a function of the temperature for the compound $\mathrm{Gd}_{4.7} \mathrm{Nd}_{0.3} \mathrm{Si}_{4}$, for two different applied magnetic fields. 80

Figure 56: The design of the support of the magnetic material plates acting as the stator of the rotary TMM, with the indication of the inlet and outlet connections of fluids. 83

Figure 57: The model of the magnetic material plate and support structure used for the CFD simulations. 84

Figure 58: The streamline of the fluid flow obtained from the CFD simulation, showing the recirculating serpentine pattern of the octagonal internal channels in the plate.

Figure 59: The stator of the rotary thermomagnetic motor, with the $\mathrm{Gd}_{4.7} \mathrm{Nd}_{0.3} \mathrm{Si}_{4}$ magnetic material plates mounted...... 86

Figure 60: The design of the hydraulic connections and hoses for the Rotary TMM, with the inlet valves directly mounted into the support of the magnetic plates, and the outlet valves fixed to the workbench. 87

Figure 61: Pressure loss curve of one magnetic plate of the rotary TMM developed, showing the measured results and the simulation corrections.

Figure 62: The temperature contours on the plate, obtained through the CFD simulation, after 15 seconds of heating. 90

Figure 63: The experimental measurement of the temperature developed in the magnetic material plate. To the left side can be seen the nylon plug connections and the control valve. 92 Figure 64: The temperature cycle developed by a magnetic plate of the Rotary TMM, the reference point represents the temperature at the center of the outer surface of the magnetic plate.

Figure 65: Relative volume of the magnetic material plate that is below the critical temperature $\left(\mathrm{T}_{\mathrm{C}}\right)$ of the $\mathrm{Gd}_{4.7} \mathrm{Nd}_{0.3} \mathrm{Si}_{4}$ material of $327 \mathrm{~K}$, obtained using the experimentally validated CFD simulation. 95 
Figure 66: The calibration curve of the linear flexure bearing (a), and the resulting calibrated measured force for the stand without any magnetic material (b).

Figure 67: The magnetic force between the permanent magnets arrangement and a Gadolinium sample, with a mass of 5,577 $\mathrm{g}$, at different temperatures.

Figure 68: The magnetic force between the permanent magnets arrangement and a sample of the $\mathrm{Gd}_{4.7} \mathrm{Nd}_{0.3} \mathrm{Si}_{4}$ compound, with a mass of $1.844 \mathrm{~g}$, at different temperatures. 100

Figure 69: The magnetic force curve for the Gadolinium for an idealized thermomagnetic motor. 101

Figure 70: The measured magnetization work of Gadolinium as a function of its temperature and the applied magnetic field change from a zero field region.

Figure 71: Model of the permanent magnets assembly with the magnetic material, used to validate the magnetic force obtained through the 3D magnetostatic simulations. 104 Figure 72: Comparison between the magnetic force simulated and that obtained using the 3D magnetostatic simulation, for the Gadolinium at $278 \mathrm{~K}$ (a) and the $\mathrm{Gd}_{4.7} \mathrm{Nd}_{0.3} \mathrm{Si}_{4}$ compound at $314 \mathrm{~K}(\mathrm{~b})$ 105

Figure 73: Analysis of the approximation between the simulated results and the measured data, in (a) the linear regression of the data, and in (b) the plotting of the residual errors from the regression. 105

Figure 74: Section view of the Rotary TMM, presenting three possible configurations of control, in (a) the three plates approach proposed initially, in (b) the six plates redesign, and in (c) the three poles configuration. 107

Figure 75: Models of the permanent magnets assembly with the magnetic material plates, used to compare a Rotary TMM motor with control configuration of three plates (a), and with six plates (b). 108

Figure 76: The torque produced by the three different configurations of control studied, obtained through the 3D magnetostatic simulation. 109

Figure 77: The built Rotary TMM, with the data acquisition system and the magnetic break used to determine the peak power output of the rotor.

Figure 78: The model of the rotor of the rotary thermomagnetic motor, used in the dynamic analyses 
Figure 79: Comparison between the measured variation of the angular position of the rotor, during a single step, from the built system and from the dynamic simulation. 111 Figure 80: In (a) the angular velocity developed by the Rotary TMM, comparing data from the simulation and measurements, and in (b) the simulated angular acceleration of the rotor.

Figure 81: The cycle developed by the Rotary TMM built, in (a) a complete rotation of the motor, and in (b) the oscillation in the position during a single step.

Figure 82: The magnetization work of the MnAs, as a function of its temperature and applied magnetic field change, for the heating (a) and cooling (b) processes. 116 Figure 83: Torque produced by the rotor of the Rotary TMM, calculated using the dynamic simulation data.

Figure 84: Change in the control design for the operation of the motor with two cold plates in the six poles configuration, adding the 'half-step' logic to increase the rotational speed.

Figure 85: An alternative control to recover the heat from the outflow of the plates of magnetic material in the Rotary TMM, in (a) the thermal cycling individually developed by the two parallel plates of the TMM, in (b) the equivalent heating cycle with the recovery of heat from the plate being cooled.

Figure 86: The die pressure mold design shown in (a) is capable of producing magnetic material plates with complex geometry and internal channels for the flow of heat exchange fluids, as shown in (B). Dimensions are shown in millimeters

Figure 87: Picture of the built pressure die mold with a $5 \times 5 \mathrm{~cm}$ scale reference. The mold was fabricated from tool steel by a specialized tooling company.

Figure 88: Picture of two of the plates produced by the compacting and epoxy bonding technique, they are composed of $20 \mathrm{~mm}$ sections (shown in Figure 86.b), connected with 3 tungsten rods and glued using epoxy, for a final length of $100 \mathrm{~mm}$. 130

Figure 89: Nickel thermal conductivity as a function of the temperature, for a well-annealed $99.99 \%$ pure nickel, with residual electrical resistivity $\sigma 0=0.038410^{-4} \cdot \Omega \cdot \mathrm{m}$. The dotted vertical line shows the Curie temperature of the material at $631 \mathrm{~K}$ 133 Figure 90: Thermal Conductivity measurement device selection depending on the thermal conductivity range (with selected materials for comparison) and the measured temperature range. 
Figure 91: A standard test method for thermal conductivity measurement using a heat flow meter, apparatus with a single heat flux transducer and one specimen ........................................... 135

Figure 92: Proposed design of the thermal conductivity measurement apparatus.................... 138 Figure 93: Picture of the built thermal conductivity measurement apparatus, with a $5 \mathrm{~cm}$ scale. 140

Figure 94: AISI 304 stainless steel recommended thermal conductivity as a function of the temperature, the estimated error is of $\pm 10 \%$ below $100 \mathrm{~K}, \pm 5 \%$ for the range from 300 to $800 \mathrm{~K}$, and $\pm 10 \%$ above $1400 \mathrm{~K}$. 142 Figure 95: Fitting of the calculated reference heat flow with the Peltier Temperature and Peltier Voltage, using the least squares interpolant, the black dots indicate the measured data points. . 144 Figure 96: Evaluation of the thermal conductivity of 304 stainless steel measured data compared to the known reference values from Touloukian et al. (1970a). 145 Figure 97: Evaluation of the thermal conductivity data measured for the Ti-6Al-4V titanium alloy, and comparison between the measured data and the available references from Touloukian et al. (1970a). 146

Figure 98: Measured thermal conductivity of the intermetallic compound $\mathrm{Gd}_{4.7} \mathrm{Nd}_{0.3} \mathrm{Si}_{4}$ as agglomerated with 5\%wt. of epoxy resin, and the fitted curve used in the CFD simulations..... 147 


\section{LIST OF TABLES}

Table 1: US Department of Energy technology readiness level scale...................................... 4 


\section{CONTENTS}

ACKNOWLEDGMENTS ................................................................................... II

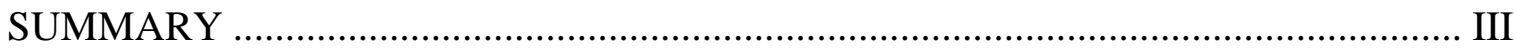

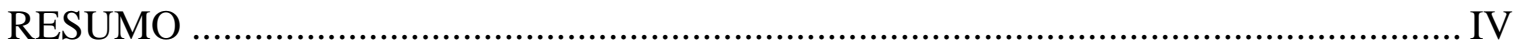

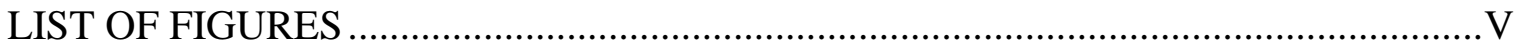

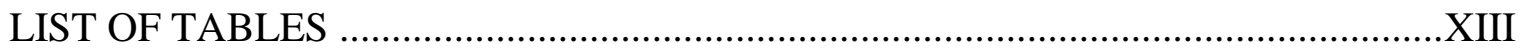

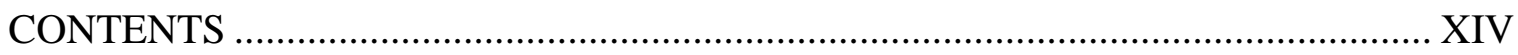

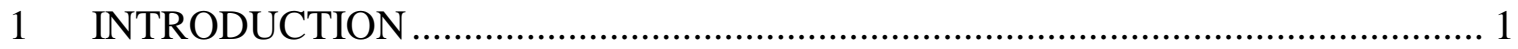

1.1 ReSEARCH OBJeCTIVES .......................................................................... 5

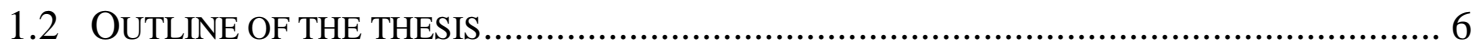

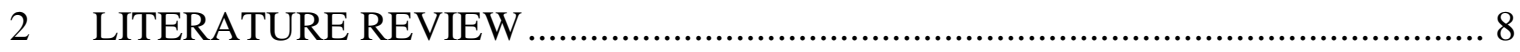

2.1 EARLY DEVELOPMENTS IN THERMOMAGNETISM............................................. 9

2.2 THE FIRST THERMOMAGNETIC ENERGY CONVERSION DEVICES ............................. 11

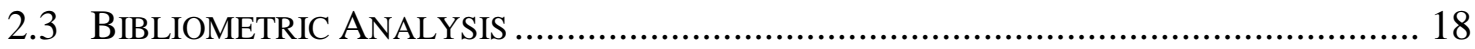

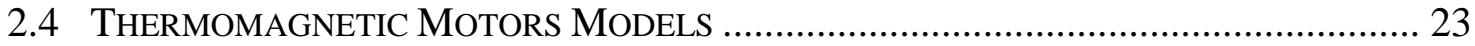

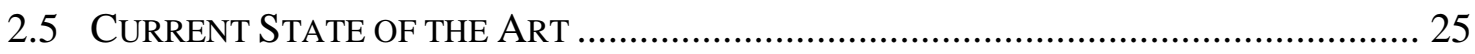

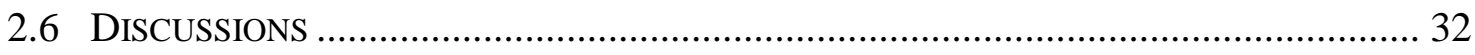

3 THERMODYNAMIC ANALYSIS OF THE THERMOMAGNETIC DEVICES ... 34

3.1 Thermomagnetic Motors Models ............................................................. 36

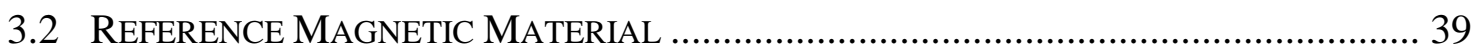

3.3 THE THERMODYNAMIC CYCLE OF THERMOMAGNETIC MOTORS ........................... 41

4 THE THERMOMAGNETIC MOTORS DEVELOPED ....................................... 48

4.1 The idealized Tesla type Thermomagnetic Motor..................................... 48

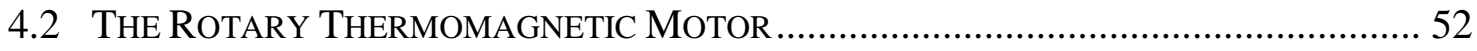

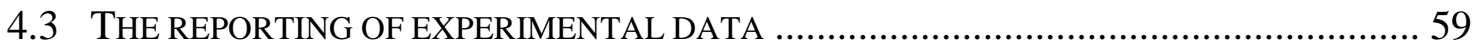

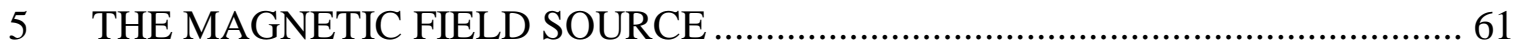




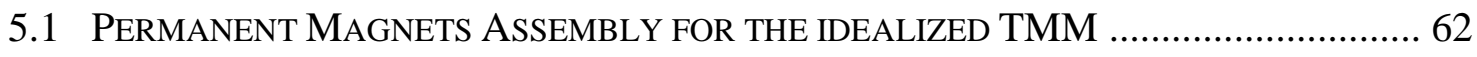

5.1.1 Experimental determination of the applied magnetic field. ........................ 65

5.1.2 Validation of the simulated applied magnetic field...................................... 71

5.2 Permanent Magnets Assembly For the Rotary Thermomagnetic Motor 73

6 HEAT TRANSFER IN THE THERMOMAGNETIC MOTORS ........................ 79

6.1 Heat Transfer In THE Rotary THERMomagnetic Motor ............................. 81

6.2 Fluid Flow In THE Rotary THERMomagnetic Motor ................................... 82

6.3 Thermal CYCLING OF THE MagnetiC MATERIAL............................................. 89

7 THE THERMOMAGNETIC MOTOR DYNAMICS ........................................... 96

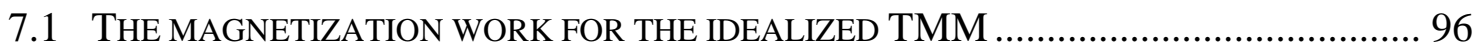

7.2 VALidation OF THE Simulated Magnetic ForCES........................................ 103

7.3 THE POWER OUtPut OF THE RotaRy THERMomagnetic MotoR ....................... 106

8 IMPROVEMENTS IN THE BUILT ROTARY THERMOMAGNETIC MOTOR 114

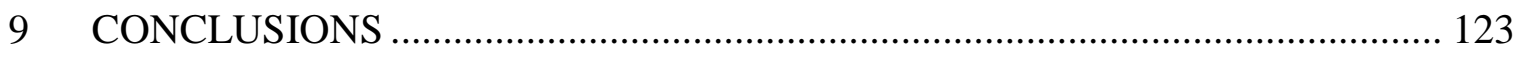

9.1 RECOMMENDATIONS FOR FUTURE WORK ................................................. 125

APPENDIX 1 - FABRICATION OF THE MAGNETIC MATERIAL PLATES ......... 128

APPENDIX 2 - THERMAL CONDUCTIVITY MEASUREMENT APPARATUS .... 131

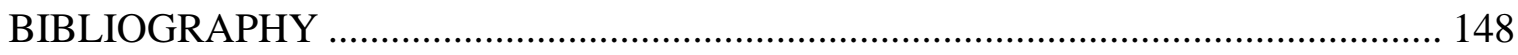




\section{INTRODUCTION}

The easy access and the steady supply of energy have constituted one of the necessary conditions for economic development. Many of the recent environmental and humanitarian challenges and disasters have a close relationship with the use and control of important energy sources. The importance of affordable and clean energy has been reaffirmed by the goal seven of the United Nations 2030 agenda for sustainable development (UNITED NATIONS, 2015), which puts the universal access to modern energy services and the substantial increase of the share of renewable energy in the global energy mix as one of the seventeen top global priorities for the next decade.

Currently, no single source or a mix of sources of energy is capable of fulfilling the need for clean and affordable energy supply for a growing global demand completely. Each of the available sources has its own economic, social and environmental costs and benefits, which strongly influence other governmental and global priorities. Brazil, for instance, has made a great effort into having a renewable-based energy matrix, with a total participation of $43.5 \%$ of the total internal energy offer composed by renewable energy, and with $81.7 \%$ of the electrical energy production composed by renewable energy, of which hydropower energy represents $61.5 \%$ (MINISTÉRIO DE MINAS E ENERGIA, 2017). At the same time, the global mean dependency on renewable energy for electrical energy production was only $26 \%$, and $41.3 \%$ for the countries members of the Organization for Economic Co-operation and Development (OECD).

Although Brazil has a highly renewable-based energy matrix, there is still much progress to be made in the use of clean energy sources. Solar energy participation represented only $0.01 \%$ of the electrical energy production, while it represents $1.6 \%$ in the mean of the OECD countries, and the use of geothermal energy had no participation percentage in Brazil, while it represents already $0.5 \%$ of the electrical energy production for the OECD members (MINISTÉRIO DE MINAS E ENERGIA, 2017). This fact represents enormous untapped potentials of energy conversion in Brazil, as both sources are available. 
Especially the abundant solar energy potential of which the advantageous geographical location and climate conditions provide a solar irradiance of $14 \mathrm{MJ} \cdot \mathrm{m}^{-2} \cdot \mathrm{day}^{-1}$ on the least exposed south part of the country and up to $22 \mathrm{MJ} \cdot \mathrm{m}^{-2} \cdot \mathrm{day}^{-1}$ on the northeastern region, comparable to the best regions in the world (AGÊNCIA NACIONAL DE ENERGIA ELÉTRICA, 2008).

Energy conversion constitutes one of the main focuses of interest and development in modern engineering. In particular, the conversion from an available disordered form of energy, meaning one that has a high entropy level such as the internal energy of matter, thermal radiation or chemical energy; into useful ordered energy, as either potential energy (gravitational, electrical or magnetic) or organized kinetic energy (KOTAS, 1985). The sustainable use of energy is intrinsically linked to the efficiency of the systems that are used to make the conversion, and the technology of these converters has a direct influence in the quality and quantity of their energy consumption, production, and also the wastes discharged to the environment (OLIVEIRA JUNIOR, 2013).

Many heat sources are currently used as part of the global energy matrix; such as the combustion of fuels, either non-renewables as some form of coal, oil, and natural gas, or renewables as biomass, biofuels, and biogas; also the heating provided by nuclear fission of radiative heavy elements; or the heat provided by geothermal sources or solar thermal radiation. According to Rosa (2012), in developed countries 90\% of the total energy production is derived from combustion processes, from which most is related to nonrenewable sources.

Most of the commonly used thermal engines operate at a high-temperature high exergy condition, such as internal combustion engines, gas turbines, and steam engines. These energy converters usually operate using a high-grade heat source, meaning that the hot reservoir temperature is usually above $673 \mathrm{~K}$ and considering a normal environment temperature $\left(T_{0}\right)$ around $298 \mathrm{~K}$ (WOOLNOUGH, 2011). Some steam engines operate using medium grade heat, with temperatures between $373 \mathrm{~K}$ and $673 \mathrm{~K}$, notably those applied in nuclear power plants, with the pressurized water reactor design whose steam temperature is usually between $473 \mathrm{~K}$ and $573 \mathrm{~K}$ (HETSRONI, 2011). Few solutions exist that operate at low temperatures, the so-called low-grade heat with temperatures below $373 \mathrm{~K}$. 
A vast amount of energy is available in the low-grade heat form, as abundant waste heat from power plants and industrial process (STRAUB et al., 2016), lower temperature (below $373 \mathrm{~K}$ ) geothermal wells, and from solar collectors (CHU; MAJUMDAR, 2012). Although this form of heat energy is so widely available, it is often the hardest to recover cost-effectively (WOOLNOUGH, 2011). Diverse technological research have looked into alternatives to feasible convert low-grade thermal energy into useful work; as through the use of organic Rankine cycles that realize the conversion using specially developed steam engine systems operating with complex thermal fluids (CHEN; GOSWAMI; STEFANAKOS, 2010; TCHANCHE et al., 2011); or using thermoelectrical converters, that realize the direct conversion from heat into electrical potential using the Peltier-Seebeck effect (CHAMPIER, 2017); or through the use of Stirling engines (KONGTRAGOOL; WONGWISES, 2003); and even through complex thermo-osmotic vapor transport systems (STRAUB et al., 2016).

However, the possibility of cost-effectively converting low-grade heat could open a new range of economic and social developments. The use of solar energy, as an example, could be significantly extended by the use of cheaper solar collectors, as in the present day, photovoltaic plates are still much more expensive than thermal-solar plates, also increasing the applicability of thermal energy storage solutions, helping to accommodate the oscillating renewable energy generation to the power grid needs (DENHOLM et al., 2015). This technology could also be applied to increase the energy efficiency of current power plants, industries, and transportation systems, through the use of the lower temperature waste heat. Currently, none of the available technology is fully capable of being used in such ways.

Thermomagnetic motors (TMM) can be presented as an alternative to convert heat into useful mechanical work. These systems use the variation on the magnetic susceptibility of magnetic materials as they are heated, modifying the magnetization of the initially ferromagnetic material as a function of its temperature. If the material is heated above a critical temperature, it loses its magnetic attraction, becoming paramagnetic or weakly ferromagnetic. Reversible, if the material is then cooled below its critical temperature, it returns to its original ferromagnetic state. The coupling of alternative heating and cooling processes with an external applied magnetic field gradient can produce mechanical work.

Materials science research has allowed, since the 1970s, the production of magnetic materials with critical temperatures close to room temperature (MURAKAMI; NEMOTO, 
1972). This fact combined with the development of high remanence permanent magnets has allowed the research in the field of thermomagnetic motors to produce some promising results recently. Despite this growing interest in the development of such motors, primarily as a new way to convert heat from low-grade heat sources, the technology still needs to be researched and developed to reach its full applicability potentials.

To understand the present development of TMM one can use the concept of technology readiness level, providing a systematic approach for measuring the maturity of a given technological proposal, and also giving a unidimensional scale to access the depth to which research is to be pursued. The idea of using technological readiness level (TRL) scales was introduced by NASA in the 70s (SADIN; POVINELLI; ROSEN, 1989), as a response to the management challenges of producing applied technologies based on new and untested innovations. The use of TRL measurements was later adopted by the US Department of Defense and suggested to many other governmental agencies, then being adapted to the specific needs of the energy sector by the US Department of Energy (SANCHEZ, 2011). The nine levels to which the level of development of new technology can be accessed are presented in Table 1.

Table 1: US Department of Energy technology readiness level scale

\begin{tabular}{ccl}
\hline $\begin{array}{c}\text { Relative Level of } \\
\text { Technology Development }\end{array}$ & $\begin{array}{c}\text { Technology } \\
\text { Readiness Level }\end{array}$ & TRL Definition \\
\hline Basic Technology Research & TRL 1 & Basic principles observed and reported \\
\cline { 2 - 4 } Research to Prove & TRL 2 & $\begin{array}{l}\text { Technology concept and/or application } \\
\text { formulated }\end{array}$ \\
\cline { 2 - 4 } Feasibility & TRL 3 & $\begin{array}{l}\text { Analytical and experimental critical function } \\
\text { and/or characteristic proof of concept }\end{array}$ \\
\hline Technology Development & TRL 4 & $\begin{array}{l}\text { Component and/or system validation in } \\
\text { laboratory environment }\end{array}$ \\
\hline Technology Demonstration & TRL 5 & $\begin{array}{l}\text { Laboratory scale, similar system validation in } \\
\text { relevant environment }\end{array}$ \\
\hline System Commissioning & TRL 7 & $\begin{array}{l}\text { Engineering/pilot-scale similar (prototypical) } \\
\text { system validation in relevant environment }\end{array}$ \\
\cline { 2 - 5 } System Operations & TRL 8 & $\begin{array}{l}\text { Full-scale, similar (prototypical) system } \\
\text { demonstrated in a relevant environment }\end{array}$ \\
\hline test and demonstration \\
\hline
\end{tabular}

Source: Sanchez (2011) 
The technology readiness assessment was originally proposed as a means to evaluate whole technological approaches, providing a complete overview of an area of research. But this methodology can also be adapted to access individual systems and designs within a given field, with the caveat that the specific evaluation of a single design will not be representative of the actual development of a technological field.

\subsection{Research Objectives}

Most of the present research in thermomagnetic motors is focused in developing the technological concept of its motors, possibly being classified between a TRL 2 and 3, while few studies have built and reported on laboratory scale technological demonstrators. With that, the most advanced research in the field of TMM can probably be classified in a TRL 4 . The main objective of this thesis is the development of a rotary thermomagnetic motor, to be used in the conversion of heat from a source at a temperature between $343-353 \mathrm{~K}$, and through that advance the technological development of such motor, from a feasibility study level up to a technological demonstration level of development.

Therefore the main objective is focused on allowing the applicability study of such motors into the conversion of low-grade heat sources, and contributing to a greater understanding of their potential advantages and challenges. To achieve this result is adopted an integrated approach of numerical simulation and experimental validation. This methodology allows a good understanding of the critical working principles of the proposed TMM, while also opens the possibility for a fast improvement process through a valid computational model.

Initially, an idealized TMM is studied using magnetostatic simulations which are then validated through the feedback provided by an experimental test stand. These initial results are used in the development of a scaled rotary type thermomagnetic motor. The design studied was previously conceptualized and patented by Coelho, Gama, and Silva (2012), which is developed both through numerical simulations and experimentally.

Great care was taken when reporting the experimental results, and necessarily some quantitative indication of the quality of the results is given as to access its reliability, allowing the comparison between measurement results and reference values, as well as the validation of numerical results. 


\subsection{Outline of the thesis}

The development of the thesis is done with a systems-based approach, without splitting methodologies and results into different chapters, although such distinction is presented for each system individually. This way both the simulation and experimental methods are reported close to their results, conceivably facilitating the understanding and readability of the text.

Chapter 2 presents a brief literature review of the development of thermomagnetic motors, aiming to provide a first principle understanding of the workings of these systems, and the different models used to study these motors in the currently available literature. A historical review is presented to provide a perspective of the current state of the art in the research of thermomagnetic motors. The concept of technology readiness level presented above is used as a guide to systematically access the maturity of the most relevant published research on TMM.

Chapter 3 presents the different working principles developed in the research of TMM, with a thermodynamics analysis approach. Through this, the advantages of the adopted design are demonstrated in comparison to other approaches, as well as some basic physical aspects of the proposed systems.

Chapter 4 will then present some details of the design and construction of two different devices, first a test stand built to understand better the basic principles and the characteristics of the technology concept adopted, and secondly, a laboratory scale proof of concept rotary type thermomagnetic motor is built. This device will be both modeled computationally and tested experimentally. With that, it can then be understood that TMM's can be divided into three basic systems: the magnetic field source, that is presented in chapter 5; the heat transfer processes involved in the change of temperature of the magnetic material, presented in chapter 6; and the system dynamics and power production, presented in chapter 7.

As the main objective proposed is to provide the development of the technological level of the rotary type thermomagnetic motor design, the use of the computational model validated in its fundamental parts in chapters 5, 6, and 7, can then be used to optimize some of the parameters involved in the construction of the motors. Proposals for these improvements are presented in chapter 8 . 
The thesis conclusions are presented in chapter 9, with the review of the validated models presented and a general perspective of the thesis and some considerations on the future perspectives of TMM into the application of low-grade heat source conversion, as well as some considerations into new applications and possible challenges.

Appendix 1 presents the process used to bond magnetic material powders with an epoxy resin, using a pressure compaction technique. This process was developed to obtain the magnetic material plates used in the laboratory scale proof of concept rotary type thermomagnetic motor built. Appendix 2 presents a spin-off experiment designed to measure the thermal conductivity as a function of the temperature of a magnetic material. This experiment was fundamental to the production of valid simulations of the heat transfer parameters of the thermomagnetic motor studied, but also provided an interesting opportunity to study different magnetic materials and their thermal properties. 


\section{LITERATURE REVIEW}

This brief literature review aims to provide a first principle understanding of the workings of thermomagnetic motors, and the different models used to study these motors in the currently available literature. A historical review is presented to provide a perspective of the current state of the art in the research of thermomagnetic motors.

In the study of the origins of current scientific developments, it is not uncommon to find misconceptions and wrongly attributed discoveries, even when relating to areas of research with a high impact and visibility. As recently demonstrated by Smith (2013) about the magnetocaloric effect, whose discovery was, since 1999 and until recently, attributed to Warburg but should, in fact, be attributed to Weiss and Piccard in their work from 1917 (WEISS; PICCARD, 1917).

The "discovery" in the natural sciences is a theme for a complex area of research, in which there seems to be a consensus that a "discovery" cannot be considered as a single historical event, but instead a "retrospective social judgment" (CANEVA, 2005). As proposed by Kuhn (1962), scientific discovery is not only correlated to a new fact but also the understanding of its meanings. As the author pointed out, "recognizing both that something is and what it is." It is, then, usually impossible to determine an exact historical moment in which the discovery took place, with the noted exception of previously theoretically predicted discoveries, like that of new elements in the periodic table or new atomic particles.

By taking these facts into account, the historical review presented in this work does not specify the single inventor or a moment of discovery of thermomagnetic motors. But rather it presents the development of this technology and the different ways in which it has been perceived and presented in the past. With that, this study seeks to show how these ideas can give insights into the basis of the development of thermomagnetic motors in the present. 


\subsection{Early developments in thermomagnetism}

The basic principle of energy conversion in thermomagnetic motors is provided by the change in the magnetization of ferromagnetic materials as a function of their temperature. The so-called thermomagnetic effect occurs when a ferromagnetic material loses its magnetic attraction when the material is heated above a critical temperature, becoming paramagnetic or weakly ferromagnetic and reversibly going back to its original ferromagnetic state when cooled down. This effect was already known and experimentally tested by Willian Gilbert in the 1600 s, as the author describes in his classic work:

Take a short piece of iron wire, and when you have brought it near to a loadstone ${ }^{1}$ it will make its way through the flames to the stone; and a needle turns no less rapidly, no less eagerly, to the loadstone through a flame intervenes than if only air stands between. Hence a flame interposed does not prevent coition. But were the iron itself red-hot, it certainly would not be attracted. Apply a red-hot iron rod to a magnetized needle and the needle stands still, not turning to the iron; but as soon as the temperature has fall somewhat it at once turns to it. A piece of iron that has been magnetized, if placed in a hot fire until it becomes red-hot, and permitted to remain for a little while, loses the magnetic power. Even the loadstone itself loses its native and inborn powers of attracting, and all other magnetic properties, if left long in fire. (GILBERT, 1893, p. 107-108)

The thermomagnetic effect was later described by Faraday (1846) for many magnetic materials. In a subsequent publication, the author described the shape of the thermomagnetic transition curve as a function of the material temperature, identifying the critical temperature in which the transition was concluded for the materials Iron, Nickel, and Cobalt (FARADAY, 1856). The works of Faraday were a reference for the work of Gore (1869), who observed the same effects described by his predecessor. Gore also observed that the heating of Iron above its magnetic critical temperature would produce a dimensional change beyond the expected for the thermal expansion, already proposing that these two variations could be correlated to a structural change in the material.

One important distinction to be made is between the thermomagnetic effect as previously described and the phenomenon of thermoelectricity. The latter refers to the way a temperature difference between two sides of a material, or material junction, can produce an electric potential; this phenomenon is also known as the Peltier-Seebeck effect. As Seebeck initially described the thermoelectric effect as a magnetic polarization due to a temperature

\footnotetext{
${ }^{1}$ The term "loadstone" refers to natural occurring ferrite permanent magnets.
} 
difference (SEEBECK, 1822), being then referred to as "thermomagnetic" in nature, since Seebeck did not realize the presence of an electrical potential, but instead measured the magnetic field induced by the produced electric current passing through a wire. This interpretation was later corrected by Ørsted, who then coined the term "thermo-electric" (ØRSTED, 1823), although many papers from the XIX century still referred to the thermoelectric effect as thermomagnetic, which may cause some confusion when looking into historical papers. In this review, all the references are related to the thermomagnetic effect as a distinct phenomenon.

The thermomagnetic transition is usually classified according to the order of the transition observed in the material. The "second-order" materials exhibit a classical second-order ferromagnetic to paramagnetic transition at a critical temperature $T_{C}$, characterized by the fact that the intrinsic spontaneous magnetization goes continuously to zero when the temperature is raised to $T_{C}$. While the "first-order" materials have a transition in which the magnetization changes "discontinuously" at $T_{C}$, with latent heat associated with the phase transition. In real systems, the first-order/second-order distinction may be blurred, due to the spreading out of the first-order transition, attributed either to the impurities and spatial variations in the stoichiometry of the material (SMITH et al., 2012), or to the dependence of the transition on a third thermodynamic degree of freedom, beyond the known dependences on temperature and induced magnetic field; then, according to the Gibbs phase rule, instead of a single point there will be a finite region of the phase (GAMA et al., 2009) 


\subsection{The first thermomagnetic energy conversion devices}

Until at least the 1870's the thermomagnetic effect was studied mainly from a magnetic materials perspective and no references were made into the possibility of using this effect as a mean for the conversion of thermal energy into useful mechanical energy. However, one must notice that the experiments of Gilbert and Faraday already used this conversion as a mean of measuring the thermomagnetic effect, that is, by heating or cooling a ferromagnetic needle and observing the change in the magnetic attraction force as a function of its temperature. This way, it would also be possible to attribute the invention of thermomagnetic motors to these authors, but since none of the previously mentioned authors refers to their experimental apparatus as means of energy conversion, or motors, this would consist an unjustified assumption of the invention.

In 1879 the researchers Houston and Thomson through their study of the thermomagnetic effect described a motor using this principle, and in a short paper presented it to the journal of the Franklin Institute (HOUSTON; THOMSON, 1879). Their motor, presented in Figure 1, uses a disk of ferromagnetic material that is fixed in a rotating axis and inserted into a magnetic field gradient provided by a permanent magnet. The disk is heated in one side and cooled in the other, producing a torque in the direction of the applied field, due to the change in the magnetic attraction force with the increase in temperature. Although these authors might be viewed as the first ones to consider thermomagnetic motors as a mean of energy conversion, they did not suppose it as having any practical value, suggesting that their motor was useful only as a demonstration of the correlation between magnetism and heat, or a mere scientific curiosity.

Figure 1: The "curious thermo-magnetic motor".

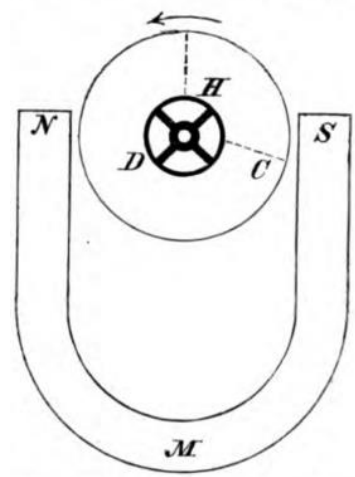

Source: Houston and Thomson (1879) 
Another pioneering thermomagnetic device was proposed by McGee (1884), his design was similarly based around a wheel of ferromagnetic material under a magnetic field gradient, and being partially heated by a Bunsen burner. The main difference between this proposal and that of Houston and Thomson (1879) is the use of a wire of ferromagnetic material to form a spoked wheel, instead of a solid disk. This system is proposed to lift a small weight and to be used as an experiment demonstrating the thermomagnetic effect.

It was only near the end of the XIX century that a real interest in the practical use of thermomagnetic motors occurred, namely the famous inventors Thomas Edison and Nikola Tesla published their researches and patents aiming to use these motors in the conversion of thermal energy. In 1887, Thomas Edison presented a talk to the American Association for the Advancement of Science, revealing the thermomagnetic motor as his newest invention (HERING, 1887), to which he referred to as a "Pyromagnetic Motor." Edison's talk was followed by the publication of a series of articles about these motors (EDISON, 1887a, 1887b; HERING, 1887; WAHL, 1887), including a front cover article presented for the magazine Scientific American, as shown in Figure 2. 
Figure 2: Cover of the magazine Scientific American from August 1887, presenting the Pyromagnetic motors developed by Edison.

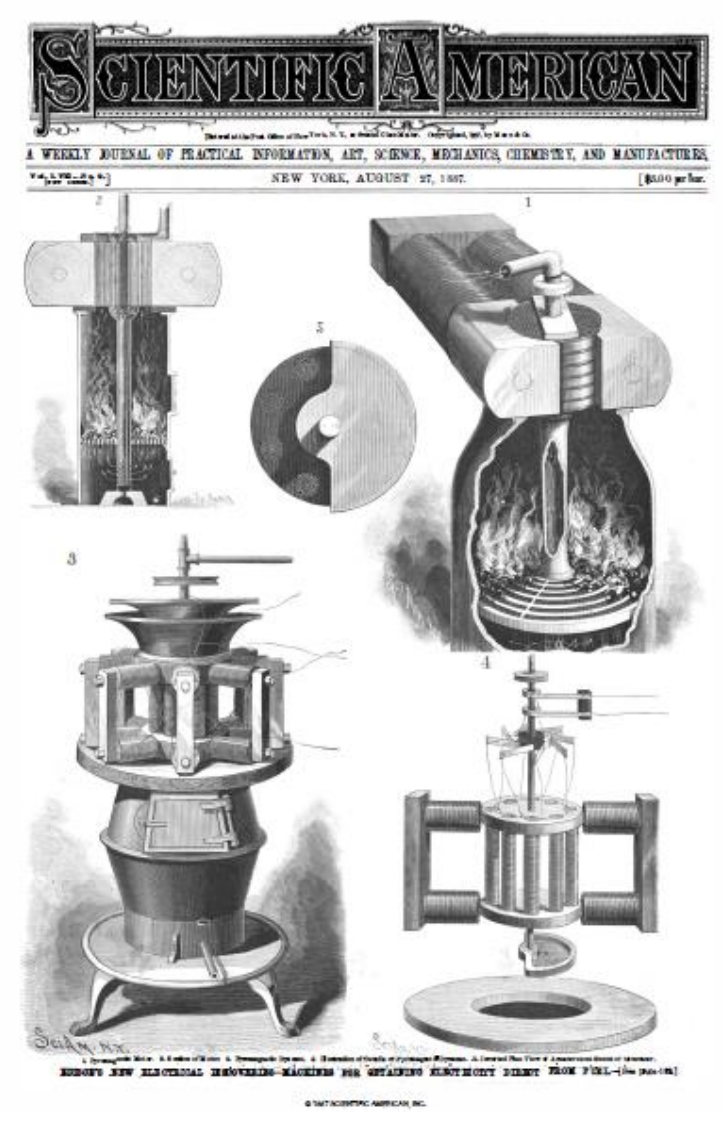

Source: Edison (1887b)

From this initial research, Edison submitted two different patents in 1887 for the design of machines that could use the thermomagnetic effect to convert available heat into useful energy. His first patent focused on the conversion of heat into mechanical energy, which he called the "Pyromagnetic Motor" (EDISON, 1888). This design uses an assembly of ferromagnetic tubes arranged into a wheel pattern, which is partially inserted into a magnetic field, and produces movement as the tubes are heated and cooled around their transition temperature, by the action of combustion gases produced by a furnace and the drafted air to combustion, as shown in Figure 3.a. This initial design is similar to that presented by Houston and Thomson (1879), shown in Figure 1, but it is focused on the applicability of the system, which could be mounted to existing heating furnaces, instead of a simple proof of concept or a mere curiosity.

Edison's second patent on thermomagnetic devices was also submitted in 1887 but was only accepted in 1892, this design was named a "Pyromagnetic Generator" and used the 
thermomagnetic effect to produce a change in the induced field around a coil, effectively converting heat directly into electricity (EDISON, 1892). Similarly to his "Pyromagnetic Motor" this generator used tubes of ferromagnetic material and the scape gases from a conventional heating furnace as an energy source, the gases alternatively heat and cool the tubes that are inserted into a magnetic field and have copper coils wounded around the assembly, as shown in Figure 3.b. As the magnetic state of the tubes changes from ferromagnetic to paramagnetic the induced field in the coils is decreased, or inversely increased, this changing field generates an alternating current, which can be used directly or converted into a continuous current using a commutator. At the time, Edison predicted that this type of thermomagnetic motor with no moving parts could be used by houses not connected to the electrical grid on remote locations.

Figure 3: Edison's patents submitted in 1887, (a) the Pyromagnetic Motor, converting heat into mechanical movement, and (b) the Pyromagnetic Dynamo, used to convert heat into electricity directly.

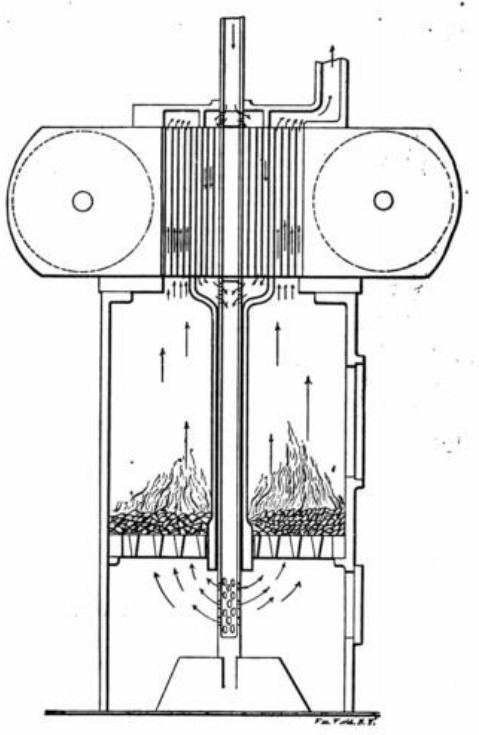

(a)

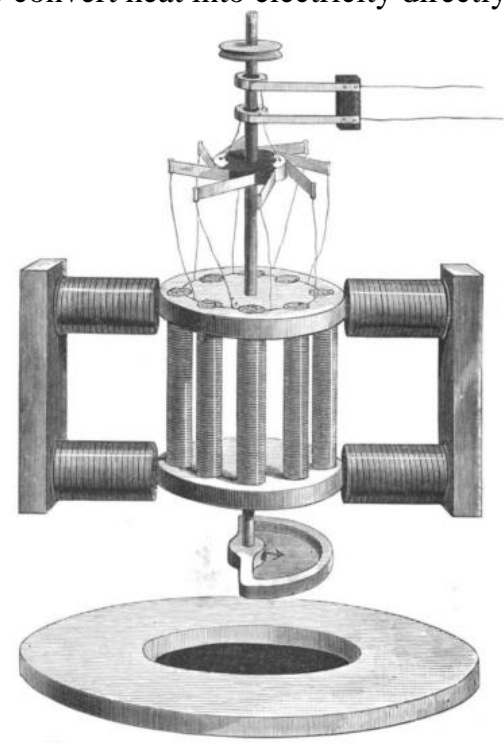

(b)

Source: Edison (1888) for (a), and Edison (1892) for (b) 
During the same period that Edison published his research and first patents on thermomagnetic devices, Nikola Tesla was also interested in the possibility of using this principle in the conversion of energy. In fact, it is possible that both researchers may have worked together in the principle of thermomagnetic energy conversion devices, according to Carlson (2013) in a recently published biography:

"It is likely that Tesla got to thinking about the relationship between magnetism and heat while working at the Edison Machine Works since Edison was then experimenting with a pyromagnetic generator that would directly produce electricity from burning coal." (CARLSON, 2013, p. 76)

In a patent submitted in 1886, Tesla presented a thermomagnetic device which he called a "Thermo-Magnetic Motor" (TESLA, 1889). This device was conceived more as proof of concept and focused on the possibility of converting heat into mechanical energy. Figure 4 presents the design patented, in it an initially ferromagnetic material (A) is fixed to a moving arm fixed to a free axis (M). The ferromagnetic material is attracted by a permanent magnet $(\mathrm{N})$ that is secured to the frame $(\mathrm{F})$. The spring $(\mathrm{W})$ is initially deformed, producing a force on the moving arm that tends to move the material away from the magnet, the tension on the spring is adjusted so that the force produced is lower than the magnetic force between the permanent magnet and the material.

In the initial state of the motor, presented in Figure 4.a, a heat source $(\mathrm{H})$ is moved to the region close to the permanent magnet, heating the ferromagnetic material (A). As the temperature of the material increases its magnetization is reduced due to the thermomagnetic effect, causing the magnetic force between $\mathrm{A}$ and $\mathrm{N}$ to also be reduced, as this force becomes inferior to the spring force, the material is moved away from $\mathrm{N}$ and also from the heat source $(\mathrm{H})$, as shown in Figure 4.b. In this second stage, the material is cooled to room temperature by the heat transfer with the environment, as the temperature of A reduces it becomes again ferromagnetic and is attracted to the initial position by the action of $\mathrm{N}$, being again heated and reinitiating the movement of the motor. 
Figure 4: Thermo-magnetic motor patented by Tesla, (a) initial position of the motor, and (b) the second stage of the movement of the motor.

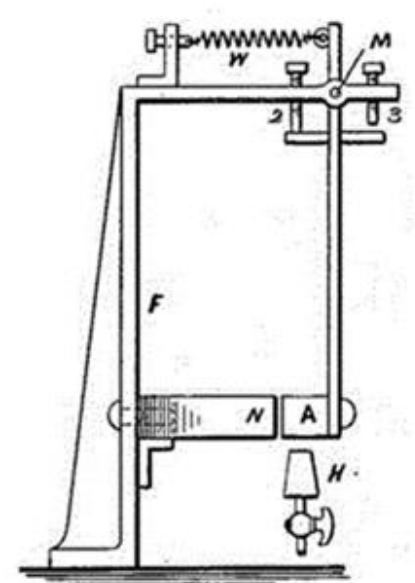

(a)

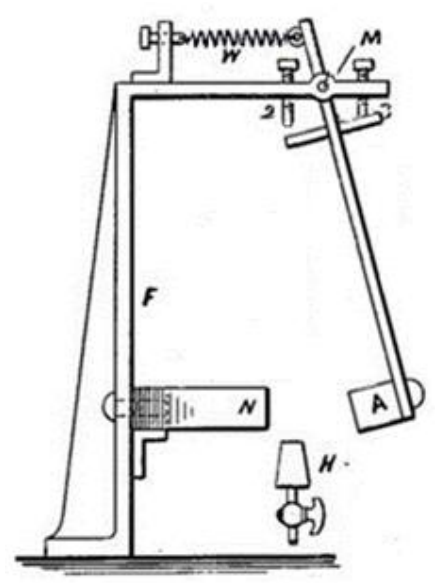

(b)

Source: Tesla (1889) for (a), and (b) adapted by the author from the (a).

In his first patent, Tesla explored different variations of the design presented in Figure 4 , including the possibility of using electromagnets or attaching a flywheel to the moving arm to obtain a rotating movement. Among these designs, he also patented the possibility of using two connected ferromagnetic materials. This way the force acting to remove the heated material away from the magnet would be produced by the action of a cooled ferromagnetic material that is attracted to another permanent magnet, eliminating the need for a spring or external force. This design is presented in Figure 5 with a top view projection. As in the design shown in Figure 4, the material is heated by a heat source $(\mathrm{H})$ and cooled to the environment, while a link (L) acts on the materials.

Figure 5: A Thermo-magnetic motor with two ferromagnetic materials linked and without the action of a spring.

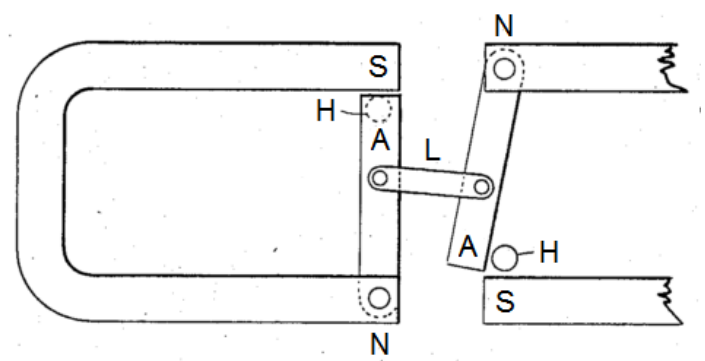

Source: Adapted from Tesla (1889)

Tesla later patented another thermomagnetic device, which similarly to Edson's Pyromagnetic dynamo (shown in Figure 3.b), proposed the direct conversion of heat into electricity, this design was called by Tesla a "Pyromagneto-Electric Generator" (TESLA, 1890). The main difference between Tesla's design and that proposed by Edison is the use of 
steam as the main heating source, with the vapor condensate being used for the cooling process.

Besides Edson and Tesla other researchers and inventors at that time had a great interest in the possibilities of converting available heat into useful energy using the thermomagnetic effect. The inventor Emile Berliner patented a thermomagnetic device for the direct conversion of heat into electricity, to be mounted into existing heating furnaces (BERLINER, 1892). In Europe, the inventor Menges, from the Netherlands, proposed a thermomagnetic device that would combine a rotating thermomagnetic motor and a generator with no moving parts, reportedly with a design more promising than that presented by Edison (SCIENTIFIC AMERICAN, 1888). Also from Europe, the engineer Hugo Bremer, from Germany, patented a thermomagnetic device based on a ferromagnetic wheel (BREMER, 1904), proposing a design similar to that developed by Houston and Thomson and shown in Figure 1. This general interest is attested by Thomas C. Martin, in a biography of Tesla first published in 1894:

"No electrical inventor of the present day dealing with the problems of light and power considers that he has done himself or his opportunities justice until he has attacked the subject of thermo-magnetism." (MARTIN, 1894, p.424)

The development of thermomagnetic devices in the turn of the XIX century can be correlated to the energy revolution that was taking place in the US and Europe, which were at that moment starting the electrification process. This initial curiosity could also be caused by the development of magnetism science since with the broader availability of electric energy the possibility of working with electromagnets became generally accessible.

Despite this great attention to the potential of energy conversion through the thermomagnetic effect, this technological promise was not fully realized at the time. Hering (1887) described some of the main challenges facing its application factors such as the low speeds obtained in the rotating systems, as the one shown in Figure 1, and that patented by Edison presented in Figure 3.a, due to the relatively slow processes of heating and cooling a material. Because of that, a system would need to be very large, in comparison to traditional dynamos, to obtain the same power outputs. There were also the materials challenges of cyclically heating a metallic material, causing deformations and high oxidation.

The materials limitations associated to the lower efficiency when competing with other energy conversion techniques in the high-temperature range, as the use of steam- 
powered turbines, required for the first prototypes to work, made the development of thermomagnetic devices limited to the area of basic research. Without any of the patented devices being effectively developed to the system operations level of research (as proposed in Table 1), or manufactured to a mass consumer market. Despite this fact, the research on thermomagnetic devices and its potentials continued through the XX century, being then stimulated by the progress on the research of new magnetic materials, and also by the advancement on the production of permanent magnets with enhanced maximum energy product $\left(\mathrm{BH}_{\max }\right)$. Which has increased by a 200 factor through the XX century, while its price per unit of energy has been reduced by a factor of 100 (OVERSHOTT, 1991).

\subsection{Bibliometric Analysis}

A systematic exploratory bibliometric analysis can be used to investigate the development of thermomagnetic devices applied to energy conversion, since its initial proposals in the late XIX century up to the currently developed research. This method of analysis is based on the search for relevant keywords in a selected database, defining clear admission or exclusion criteria to tabulate all the available papers that are in principle relevant to the study, collecting not only the metadata of these papers (Title, Author, and Year of Publication) but also the citations indexes and with that the works that are cited within the tabulated papers. The produced spreadsheet can then be used to perform a statistical and Social Network Analysis (SNA). While this exploratory bibliometric analysis does not guarantee that all the published research will be analyzed, as in fact, no review method can guarantee, it provides a basic overview of the research field and reveals the most relevant and recognized research within the field studied.

To perform such systematic review the first step is to define the relevant keywords and exclusion rules applicable. Many different terms have been used to define the thermomagnetic devices researched; this is a complicating factor into performing a complete and valid analysis, as previously shown in the beginning both the terms Thermomagnetic and Pyromagnetic were used. Also, such devices were defined as Motors, Engines, and Energy

Harvesters. Since these systems used the magnetic force interaction to produce work, the term Magnetic Power/Energy conversion is also relevant. 
The exclusion rules selected the terms relative to thermoelectricity, due to the initial confusion between thermoelectricity and thermomagnetism. The papers strictly related to material sciences were also excluded from the analysis, that is, the papers that are focused on the development and characterization of thermomagnetic materials instead of on the development of devices. Since many of the papers focused on the development of devices for energy conversion also present materials information and some form of characterization, this exclusion rule must be applied a posteriori. The final exclusion rule was that of devices that propose the use of the thermomagnetic phenomenon into information recording applications, as these systems cannot be correlated to the application of energy conversion.

The next step in performing the systematic review is to define the database to be used in the research. For this analysis, the Web of Science database was defined as an ideal source, due to its availability, ease of use to define research phrases using keywords and Boolean operators, and the possibility of exporting the selected results into a tabulated format that allows the use of a computational tool to perform the statistical and SNA. After using the defined search terms and exclusion rules, the following search phrase is applied:

("Thermomagnetic" OR "Thermo-magnetic" OR "Pyromagnetic" OR "Magnetic") AND (“Device” OR “Motor” OR “Engine” OR “Energy Harvesting” OR "Energy Conversion” OR "Power Conversion") NOT ("Recording”)

The first analyses of the results demonstrated the need to add a search term relative to the so-called "Curie Motors." This nomenclature is used to define thermomagnetic devices in a part of the literature, as the work in the motor is performed while the material is cyclically heated and cooled around its critical temperature of the thermomagnetic transition, which is commonly referred to as the "Curie Temperature". With that, the new search phrase becomes:

("Thermomagnetic" OR "Thermo-magnetic" OR "Pyromagnetic" OR "Magnetic" OR “Curie”) AND (“Device” OR “Motor” OR “Engine” OR “Energy Harvesting” OR “Energy Conversion" OR "Power Conversion" OR "Wheel”) NOT ("Recording")

Although the term Curie Temperature can be regarded as a tribute to Pierre Curie due to his important contributions to the development of science, and the fact that his Ph.D. Thesis was focused on the study of the thermomagnetic phenomenon (CURIE, 1895), it is incorrect to attribute him the discovery of thermomagnetism. As previously shown, this 
effect had already been described centuries before, and even Curie himself cited the works of Faraday and Gore in his thesis, despite this many biographies of Pierre Curie still attribute such discovery to him, including Lucibella (2014) and even his biography in the Nobel Prize Lectures (NOBEL MEDIA, 2014).

It is important to notice also that Curie himself never published or researched the possibility of applying the thermomagnetic effect to energy conversion applications, as can be seen in the publication of his complete works (CURIE, 1908).

The results of the search phrase produced, after the application of the defined exclusion rules, a total of 74 papers from the Web of Science database; these papers collectively cite additional 719 references. The high volume of data is analyzed through the use of the computational statistical and network analysis interface tool developed by Knutas et al. (2015). This tool provides an overview of the most cited papers from the searched results and their relevance within the field. One of the more direct results from the analysis is to produce a histogram of the development of the research for thermomagnetic materials, which is obtained by analyzing the year of publication of the searched papers and is presented in Figure 6.

Figure 6: Historical development of the research of Thermomagnetic devices for energy conversion, in the graph each bar plot represent a 5 years period.

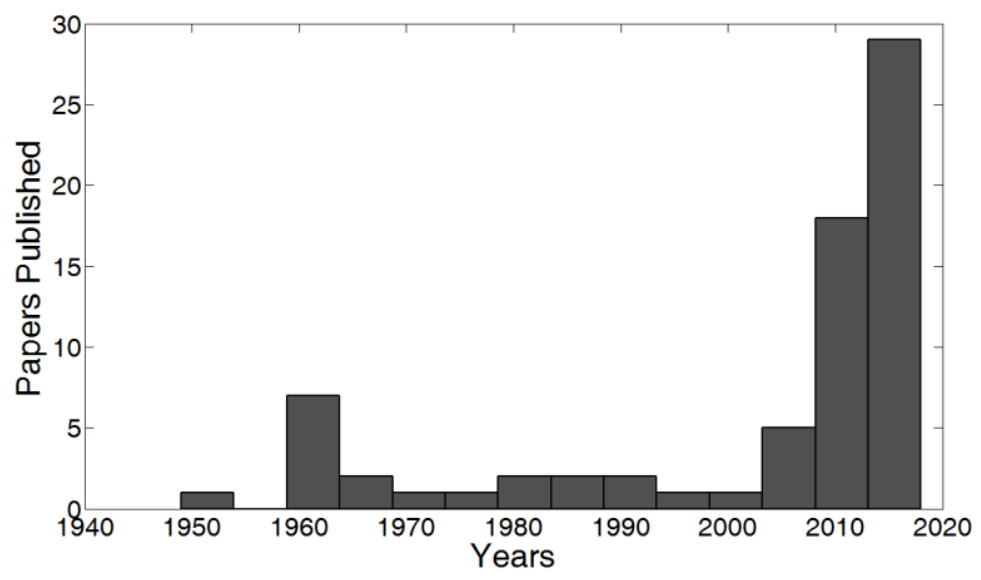

The histogram presented in Figure 6 indicates a constant mild interest through the XX century, with an interesting spike of papers published in the 1960's, and a clear growth of this field in the 2000's. Although the number of papers obtained in the systematic bibliometric search may lack a few publications that may not include the selected keywords 
or that are not included in the database, the trends show a clear representation of the advancement of the research of thermomagnetic devices.

Using the network analysis interface tool (KNUTAS et al., 2015), the papers obtained in the systematic bibliometric search and cited references were tabulated as nodes within a network, with the citations between each node presented as edges. By using the Gephi Open Source network mapping software (BASTIAN; HEYMANN; JACOMY, 2009), these results can be visualized within an interactive graph, allowing the easy identification of the most relevant research within the field of thermomagnetic motors.

The first mapping of the obtained results is shown in Figure 7, each node represents a paper either in the searched results or that is cited by them, and the arrows indicate the citation, with the direction indicating the papers cited. The size and color of the nodes represent the number of times it is cited within the results, the IN-degree level within the field, with the most cited papers being represented by a larger node with a darker color shade. The colors of the arrows indicate different clusters of papers that are cited by a source. The proximity of the clusters indicates the correlation between cited sources, while clusters that are completely isolated are an indication of adjacent research topics that are not in direct correlation with the rest of the field. 
Figure 7: Network analysis mapping of the systematic bibliometric search of the paper related to the field of thermomagnetic energy conversion within the Web of Science database.

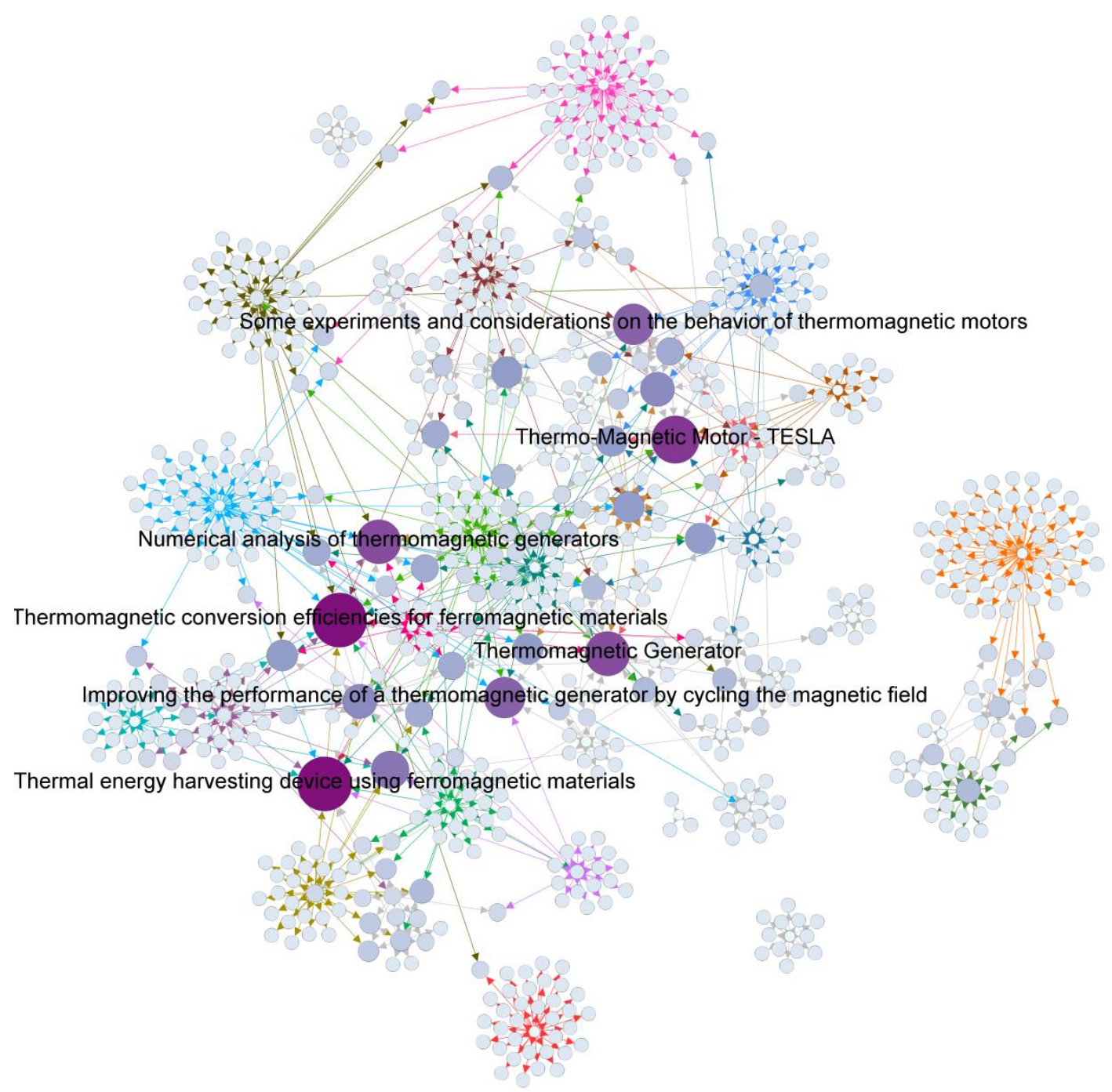

A first analysis that can be obtained from the map is to evaluate the most cited sources within the search results. In Figure 7 the title of the seven most cited papers is presented; these papers have an In-degree level of at least 9. Notably, the "Thermo-magnetic motor" patent by Tesla (1889) is cited by 11 different sources, with many sources regarding him as the main inventor of the thermomagnetic devices.

An other important analysis is provided by evaluating the total degree of correlation of a paper, meaning the sum of citations it makes and the total of times it is cited within the field, this indicates the most relevant papers within the research field. By observing the clustering of the nodes, different approaches to the thermomagnetic energy conversion 
technology can be identified, as well as the relevance of certain approaches to the development of the research field as a whole. These clusters are formed by commonly citing sources referencing to a small number of references, grouping the nodes in the network analysis mapping.

\subsection{Thermomagnetic Motors Models}

One important approach identified is that of using purely theoretical models, as seen in the work of Brailsford (1964), who proposes a design for a thermomagnetic device similar to that presented in Figure 1, as shown in Figure 8.a, and by investigating the magnetization of different ferromagnetic materials calculates a maximum theoretical efficiency of such device, though only for small applied magnetic field strengths. Another relevant theoretical model was used by Hsu et al. (2011), in which an idealized thermomagnetic cycle is proposed, as shown in Figure 8.b, from which the authors proceed to investigate the theoretical efficiency of thermomagnetic devices and propose alternatives to improve their performance.

Figure 8: Two different theoretical models used to investigate the basic principles and formulate the efficiency of thermomagnetic devices, in (a) a "ferromagnetic heat engine" proposed by Brailsford (1964), and (b) an "idealized thermomagnetic cycle," proposed by Hsu et al. (2011).
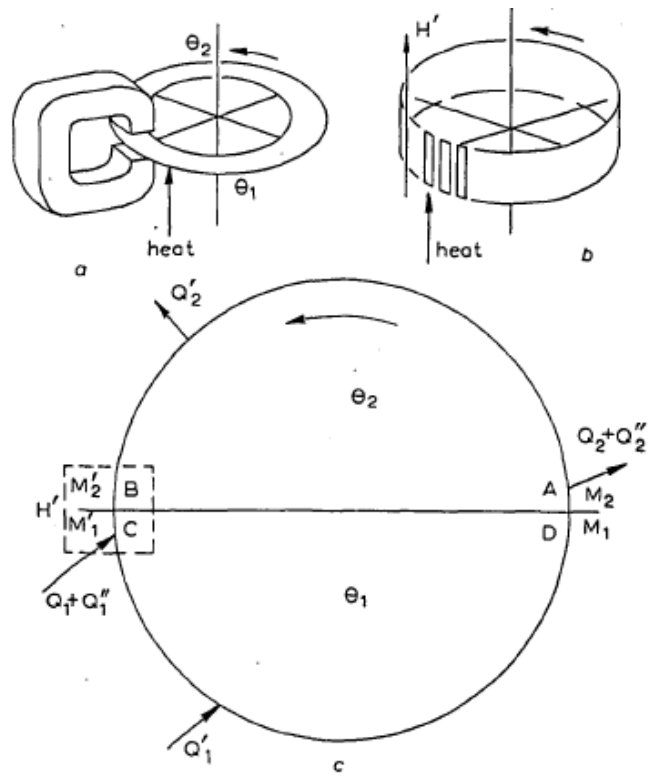

(a)
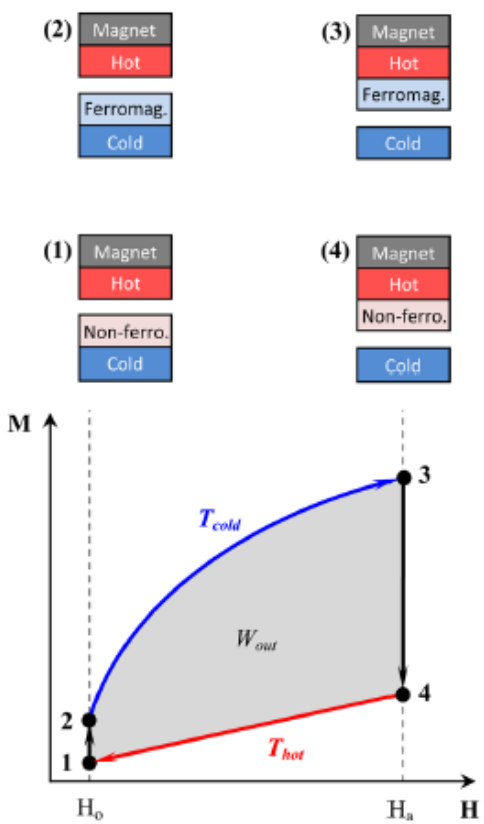

(b)

Sources: Brailsford (1964) for (a), and Hsu et al. (2011) for (b). 
A third theoretical model design was used by Brillouin and Iskenderian (1948) to study a thermomagnetic device for the direct conversion of heat into electricity, without the movement of the magnetic material, as presented in Figure 9. This model is supposed to characterize a system similar to the one patented by Edison (1892), from which the authors intended to define its thermodynamic cycle. Similar models of thermomagnetic generators were used by Elliott (1959), who also proposed to use Gadolinium as a shunt material, and Kirol and Mills (1984), who then realized a numerical analysis and calculated the power output and maximum efficiencies of those devices for different magnetic materials. More recently, a similar numerical approach was used by Almanza et al., (2017), estimating the efficiency and power output of a proposed thermodynamic cycle for thermomagnetic devices, using a magnetocaloric material as active substance.

Figure 9: The thermomagnetic generator with no movement of the magnetic materials.

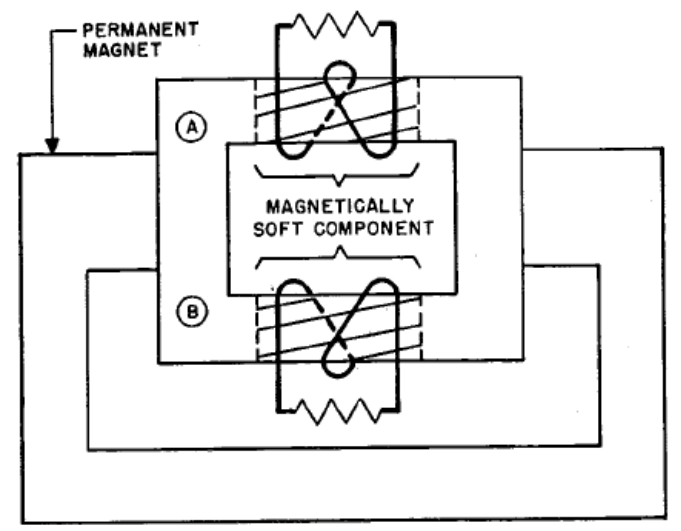

Source: Brillouin and Iskenderian (1948)

In the work published by Vuarnoz et al. (2012), the feasibility of thermomagnetic energy conversion is studied using a solely thermodynamic theoretical approach, although the research makes some optimistic assumptions into the maximal frequency of operation obtained in the motors, it concludes that the efficiency of these devices might be superior of that obtained by conventional thermoelectric systems or even organic Rankine cycles operating in the same temperatures, and also a first principle economic analysis provides an optimistic result.

Studies of thermomagnetic devices that use computational and numerical modeling compose another relevant class of theoretical studies, while not yet developing physical prototypes, these computational techniques tend to present a more direct physical approach. 
As an example, the paper published by Egolf et al. (2009) uses a multi-physics computational analysis tool to investigate the propagation of heat in a rotating disk of magnetic material, for a motor model similar to the one presented in Figure 8.a, from which they then derived promising calculations of the power output and efficiency for these type of devices. Another interesting example is the work done by Alves et al. (2013), where numerical modeling for a thermomagnetic device was implemented using MATLAB, from which a design for a prototype was proposed.

These theoretical and numerical approaches are fundamental to the progress of the understanding of the basic principles of thermomagnetic devices. They help to formulate the technological concept, and to develop the feasibility of the technological development. Despite this fact, without the experimental demonstration of these devices the technology readiness level, going back to the levels proposed in Table 1, is limited to the research level, with any such theoretical only studies then limited to a maximum TRL 2. For this reason, this review is then focused on the published research on thermomagnetic devices that present some form of experimental analysis, technological demonstrator or prototype. By using the network analysis mapping tool, the most relevant papers that satisfy such criteria can be identified.

\subsection{Current State of the Art}

In the paper "The thermomagnetic Curie-motor for the conversion of heat into mechanical energy," Karle (2001) describes the modeling of a device with a construction similar to the presented in Figure 8.a, and calculates a theoretical power output and maximum efficiency for such device. While the theoretical modeling does not present promising results, due to a calculation of the magnetization work using the energy density of the magnetic field, instead of the co-energy density, an assumption that would only be correct for linear magnetic material, as is going to be explained in chapter 3 of this thesis. Karle (2001) presents an experimental evaluation of the device modeled, though without reporting experimental data results, but concluding that the experiments agreed with the theoretical analysis.

A thermomagnetic motor based on a disk of magnetic material was also reported by Palmy (2007), in his design the magnetic material levitates inside the magnetic field 
produced by two permanent magnets, using diamagnetic disks (Bismuth) to stabilize the levitation, while the magnetic material is heated by a light source, this device is shown in Figure 10. In the paper, the rotation speed of the disk of magnetic material is reported, and a free body diagram is representing the forces acting on the disk is proposed. This work, however, is not much focused on the energy conversion aspect of the thermomagnetic motor, but rather used as a demonstration of physical phenomena involved in such system.

Figure 10: The levitating thermo-magnetic wheel built by Palmy (2007), in (a) the design of the system, with two lifting magnets (B), Bismuth discs (Bi), the floating magnet (Fm) and a radiation beam heating Fm. The built system is presented in (b).

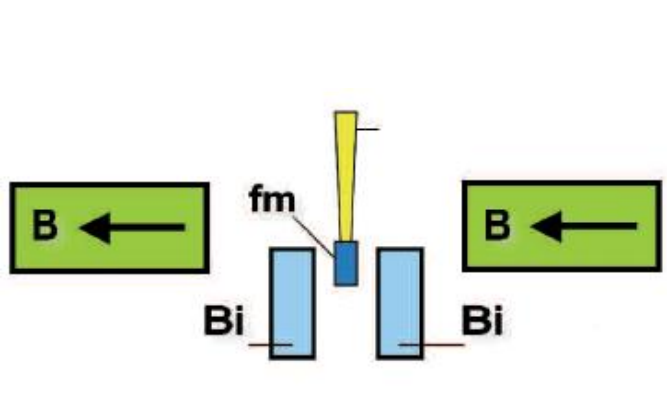

(a)

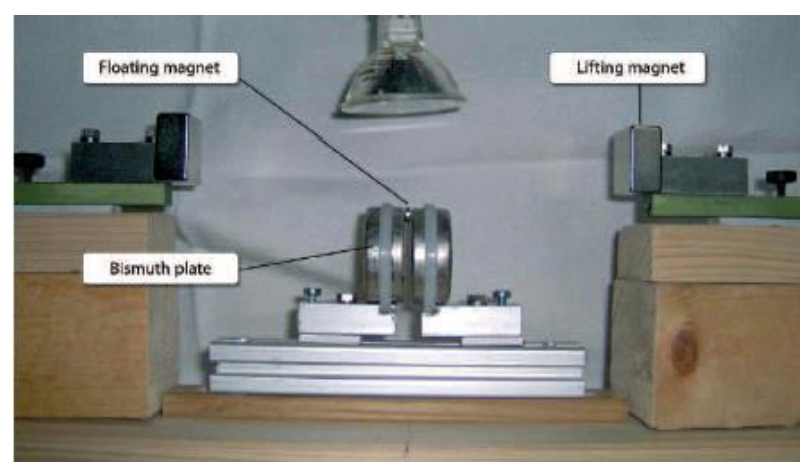

(b)

Source: Palmy (2007).

While providing interesting insights of the working principles of these thermomagnetic motors, the paper published by Karle (2001) and Palmy (2007) do not present a clear technological approach to the development of these devices. The research is instead focused on proving the feasibility of the concepts, therefore both the devices could be classified into the scale of technology readiness level as a TRL 2.

In the paper "Some experiments and considerations on the behavior of thermomagnetic motors," presented by Murakami and Nemoto (1972), the authors explore more practically the applicability of these thermomagnetic devices into the production of mechanical work from low-grade heat sources. Their device, shown in Figure 11, uses the wheel of magnetic material with a critical thermomagnetic transition temperature of $323.15 \mathrm{~K}$, being locally heated and cooled, while partially inserted into a magnetic field gradient. The applied magnetic field intensity is not reported by the authors, though they report using a room temperature electromagnet to generate the magnetic field gradient in the motor, and therefore small applied magnetic field strengths are to be expected. With that the 
torque and power output of their motor were small, to which the author report an "extremely low value" efficiency. Despite these results, an application as a control device is suggested.

Figure 11: The thermomagnetic motor built and analyzed by Murakami and Nemoto (1972).

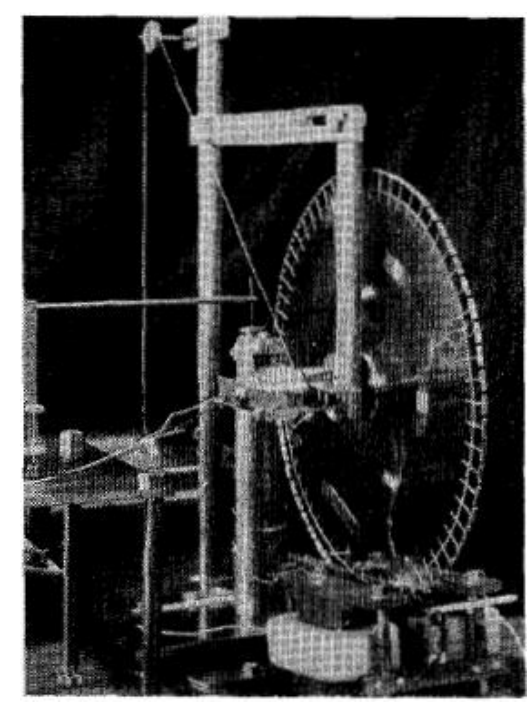

Source: Murakami and Nemoto (1972).

A more technologically developed design of a thermomagnetic motor using a disk of magnetic material was studied by Takahashi, Matsuzawa, and Nishikawa (2004). Their design, shown in Figure 12.a, is composed with three permanent magnets arrangements, with the magnetic material being the $\mathrm{Fe}_{68.5} \mathrm{Ni}_{31.5}$ alloy and a heat source at the temperature of $368.15 \mathrm{~K}$ and a heat sink at $284.15 \mathrm{~K}$. In their work the experimental procedures are reported, obtaining the power output and torque produced by the system, as well as estimations of the losses due to the hydraulic interaction, friction and the Eddy current breaking due to the movement of the conductive magnetic material inside a field. In another study (TAKAHASHI; YAMAMOTO; NISHIKAWA, 2006), a similar motor design was reported, using a cylinder of magnetic material instead of the disks previously reported. In this motor, shown in Figure 12.b, the magnetic material used is the alloy $\mathrm{Fe}_{54} \mathrm{Ni}_{36} \mathrm{Cr}_{10}$, while maintaining the same design presented in Figure 12.a, and the same temperatures of heat sink and source. Similarly, the experimental procedure is adequately described, with the power output and torque obtained by the motor reported.

The comparison between the two thermomagnetic devices built reveals that the power output increases for the motor that uses disks of magnetic material, from a maximum of 3.7 $\mathrm{W}$ for the motor using a solid cylinder magnetic material to a maximum of $6.0 \mathrm{~W}$, for the 
same rotation speed of $0.4 \mathrm{~Hz}$. The authors attribute this difference to the reduction of the Eddy current breaking losses.

Figure 12: The model of the thermomagnetic motor (a) using a disk of magnetic material to produce work, and a picture of the built concept demonstrator (b) of the thermomagnetic motor using a cylinder of magnetic material.

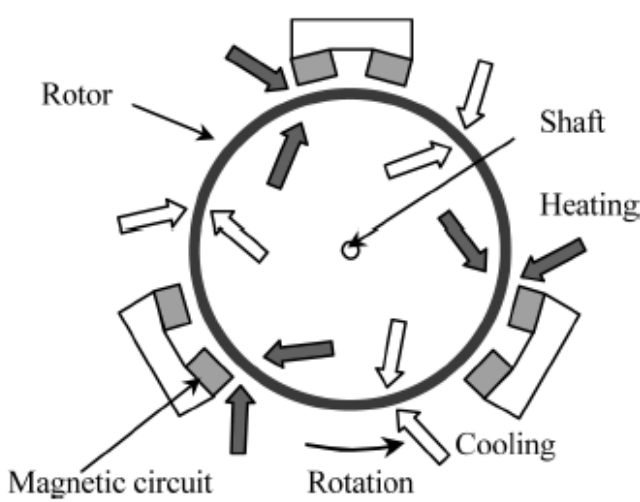

(a)

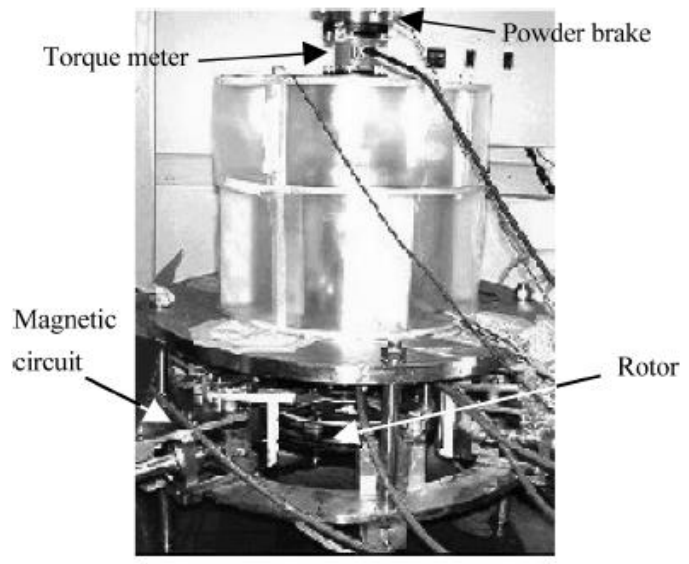

(b)

Source: Takahashi; Matsuzawa and Nishikawa (2004) for (a), and Takahashi; Yamamoto and Nishikawa (2006) for (b)

The studies published by Takahashi et al. presented a solid experimental and practical approach, showing an important advancement in the scale of the technological readiness level, from a purely conceptual level to an analytic and experimental one. From their research, the maturity level of the technology of energy conversion using the thermomagnetic devices could be classified to a TRL 3, though without focusing on the validation of this system into a relevant application environment. The relative level of technological development is still within the proof of feasibility stage.

A different approach for the design of thermomagnetic motors that can be identified in the literature is that of using cyclical heating and cooling of a bulk of magnetic material, producing an alternating mechanical movement in the interaction with an applied magnetic field gradient. This approach is more similar to the patent proposed by Tesla (1889), as shown in Figure 4, and to the modeling shown in Figure 8.b. One relevant characteristic of these designs is that the dimensions of the magnetic material can be greatly reduced. With that, it is possible to achieve increased operating frequencies as the heat transfer process is accelerated. 
The paper "Thermal energy harvesting device using ferromagnetic materials" by Ujihara, Carman, and Lee (2007) is included among the most cited references and proposes the use of a thermomagnetic device with small dimensions, to be used in low power energy harvesting applications. They built a prototype using a thin film Gadolinium strip (127 $\mu \mathrm{m})$ with their working material obtaining a frequency of $28 \mathrm{~Hz}$. The energy conversion from the produced mechanical power to a desirable electrical power is done using a piezoelectric material. The device built and its working principle are presented in Figure 13. As the authors were capable of developing the technology to an experimental proof of concept and characterization their device can be categorized into a TRL 3.

Figure 13: The thermomagnetic device with small dimensions focused on low power energy harvesting developed by Ujihara, Carman and Lee (2007), in (a) the actual device built with dimensions of $2 \cdot 10^{-2} \mathrm{~m} \mathrm{x} 2 \cdot 10$ ${ }^{2} \mathrm{~m} \mathrm{x} 6 \cdot 10^{-3} \mathrm{~m}$, and in (b) the cross-section of the device during the cooling (higher image) and heating (lower image).

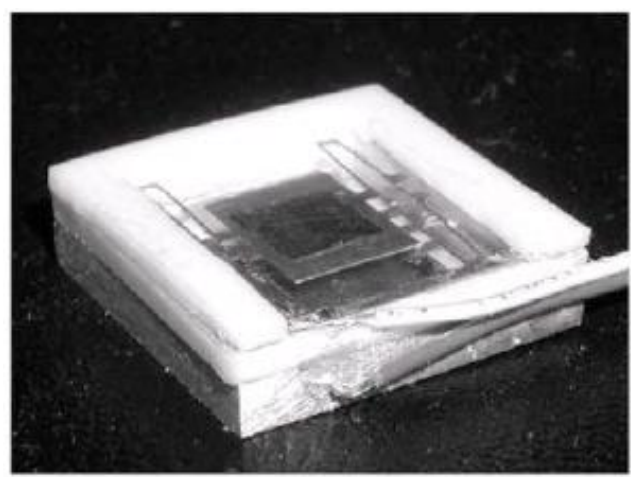

(a)

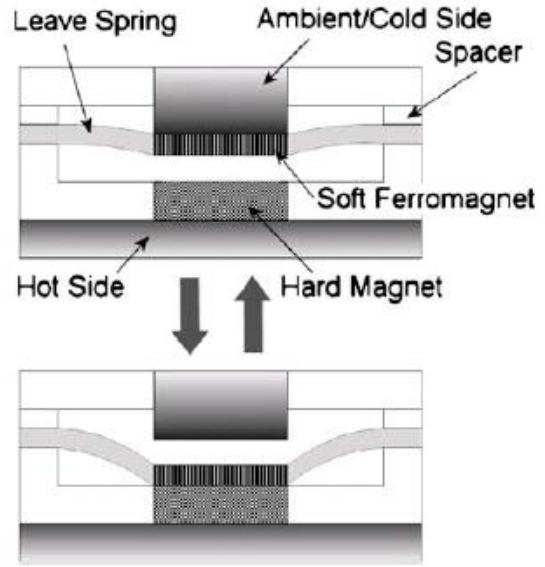

(b)

Source: Ujihara, Carman, and Lee (2007)

A similar device was reported by Chun et al. (2017), though having larger dimensions and obtaining lower operating frequencies of 1 to $3 \mathrm{~Hz}$. Their device, shown in Figure 14, uses the same magnetic material as the one proposed by Ujihara, Carman and Lee (2007), a commercially available thin film Gadolinium strip $(127 \mu \mathrm{m})$, while using Polyvinylidene Difluoride (PVDF) bimorph cantilevers as the piezoelectric material that converts the mechanical movement into an electrical current. In their initial study, Peltier plates were used to provide the heat transfer to the material under controlled conditions, from which the power output and efficiency of the device were reported.

An important differential of the thermomagnetic device developed by Chun et al. (2017) is that the authors suggest an application for the device, and test its functionality, for 
the recovery of heat from a computer CPU, showing that the device is capable of producing electrical energy from the wasted heat. From that, it can be concluded that the relative level of technology readiness of the device built is within the technology development stage, from Table 1. Since an application for the device built was proposed, as well as the validation of the technology in a relevant environment, this device can be categorized to a TRL 4.

Figure 14: A thermomagnetic-electric generator is operating through the phase transition occurring in a magnetic material during periodic cooling and heating, in (a) a schematic representation of the device, and in (b) a picture of the built device.

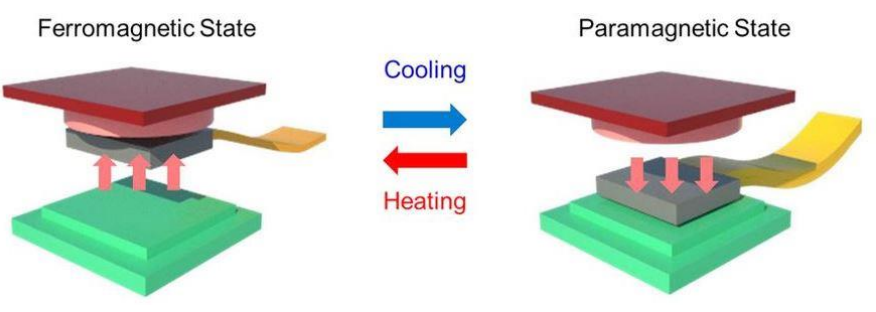

(a)

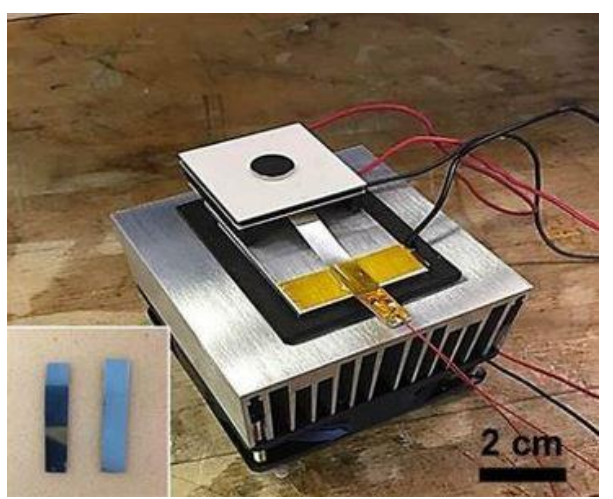

(b)

Source: Chun et al. (2017),

On the extreme of these thermomagnetic energy harvesting devices with small dimensions is the "Miniature magnetic-piezoelectric thermal energy harvester" reported by (CHEN et al., 2015). The device, shown in Figure 15, uses a similar principle of heat transfer by solid contact, like the ones shown in Figure 13 and Figure 14. With the conversion from the mechanical movement to a measurable electric current done using a piezoelectric material, with the difference that in this design the magnetic material itself acts as a spring, as presented in Figure 15.a. The remarkable characteristic of this system, however, is its miniaturized dimensions of $6 \times 3.5 \times 3 \mathrm{~mm}$, as can be seen in Figure 15.b the device itself is smaller than a one cent coin. The authors present an idea of application of such miniature energy conversion devices into providing electrical energy for wireless micro-sensors. As the authors were capable of developing the technology to an experimental proof of concept and characterization this device can be categorized into a TRL 3. 
Figure 15: The miniature magnetic-piezoelectric thermal energy harvester studied by Chen et al. (2015), with the modeling (a) and an experimental device built (b).

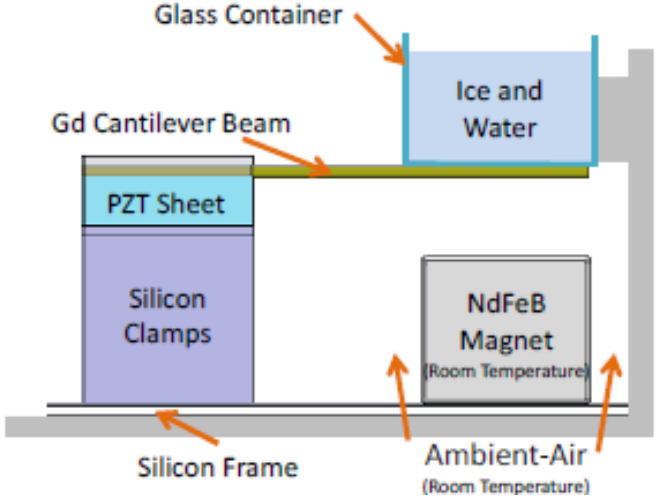

(a)

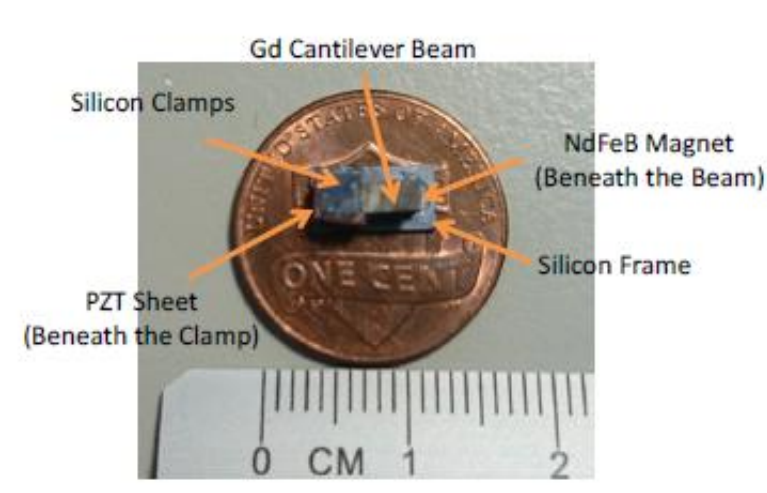

(b)

Source: Chen et al. (2015)

The third type of thermomagnetic device that can be developed is that of systems that present the direct energy conversion from heat into electrical energy, as presented in the original patent by Edison (1892), shown in Figure 3.b, and similar to the model presented in Figure 9. These "thermomagnetic generators" have been extensively studied from a theoretical standpoint (BRILLOUIN; ISKENDERIAN, 1948; ELLIOTT, 1959; KIROL; MILLS, 1984; SOLOMON, 1988, 1991). Though few devices were built and tested, with the notable exception of the study done by Christiaanse and Brück (2014), who developed the design proposed by Brillouin and Iskenderian (1948). Initially, the design was optimized using a finite element numerical analysis, and then the thermomagnetic generator was constructed, using $(\mathrm{Mn}, \mathrm{Fe})_{2}(\mathrm{P}, \mathrm{As})$ as a magnetic material with varying critical temperature transitions $\left(\mathrm{T}_{\mathrm{C}}\right)$ of $300,304,307$, and $310 \mathrm{~K}$. Their design and a picture of the built system are presented in Figure 16.

Figure 16: The static Thermomagnetic generator studied by Christiaanse and Brück (2014), in (a) the design model, with the red cylinder indicating the heated magnetic material and the blue cylinder the cooled material, in (b) the magnetic material regenerators built, with the power producing coils.

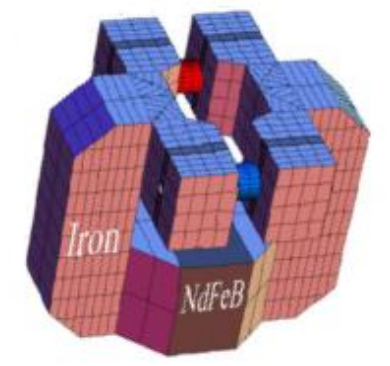

(a)

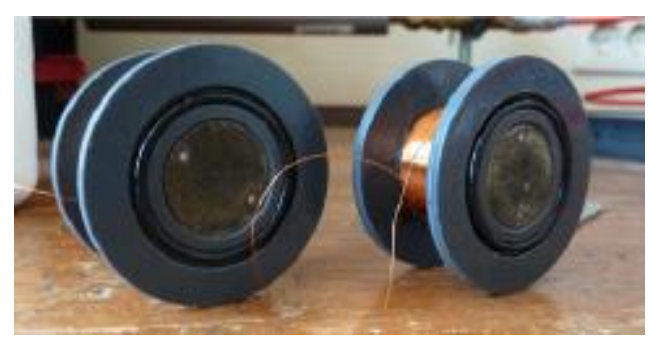

(b)

Source: Christiaanse and Brück (2014). 
In the static thermomagnetic generator developed by Christiaanse and Brück (2014), two regenerators are alternately heated and cooled by the flow of water through internal channels. The magnetic circuit formed with the NdFeB permanent magnet is closed through the colder material, and as the temperature of the materials are inverted, and their magnetization changed, a change in the magnetic field around the power producing coils occurs. The voltage obtained in the coils had a peak at $3 \mathrm{mV}$, with a frequency of $0.17 \mathrm{~Hz}$, which resulted in a maximum average power output of $0.05 \mathrm{~mW}$. The authors then attributed these low output results to the fabrication process of the magnetic regenerator materials, which was conducted using a laser technique, and probably compromised the expected physical properties of the material. In comparison between their simulation results, the actually developed power output was 800 times lower. Due to the yet limited research and not initially promising results the level of development of the design proposed can be defined as a TRL 2.

\subsection{Discussions}

From the analysis of the most relevant published research on thermomagnetic devices, identified using a network mapping analysis of the systematic bibliometric review, it can be concluded that relative level of technology development of these devices is mostly within the research to prove feasibility. Some notable exceptions can be observed because some authors are attempting to apply these systems to a more advanced technological development level. However, one important consideration to be made is to question the completeness of the systematic bibliometric review, done within the limited Web of Science database. In the sense that the database does not comprehend an ample number of the research done on thermomagnetic devices, that are published in the form of patents.

The patents constitute an important reference to the work done on thermomagnetic devices, notably the network analysis rank the patents by Tesla $(1889,1890)$ and Edison (1888, 1892) among the most cited sources for the bibliometric review conducted. The literature review presented here has a more academic focus and does not intend to provide a complete presentation of the work done in the development of the thermomagnetic devices. A bibliometric review that included these patents would be difficult to produce, due to the lack of conformity between the different patenting offices and the common poor citation 
practices observed in the patents. It's noticeable nonetheless that the International Patent Classification (WIPO, 2018) presents a specific class to define thermomagnetic devices (IPC class H01L37/04).

From the literature review, three different design approaches of thermomagnetic devices were defined. In the first category are the "thermomagnetic motor" which are proposed to convert available heat into useful mechanical energy, these motors can be subdivided into two types. The first is that of the devices that use magnetic disk material, locally heated and cooled, similarly to the first patent on these devices proposed by Edison (1888), as the one shown in Figure 12. The second type of thermomagnetic motor defined is that were the magnetic material is wholly periodically heated and cooled, with the production of an alternating movement by the interaction with a magnetic field, similar to the patent of Tesla (1889), like the device shown in Figure 14. Therefore, suggesting the use of the categorizations "Edison type" and "Tesla type" to refer the two common designs of thermomagnetic motors. The third type of thermomagnetic device refers to an entirely different approach to energy conversion, with the heat being directly converted into electrical energy; these devices are then named "Thermomagnetic generators."

This thesis is mostly focused on the development of the Tesla type of thermomagnetic devices, since they present a more promising perspective of energy conversion, as is going to be presented in the following chapter. 


\section{THERMODYNAMIC ANALYSIS OF THE THERMOMAGNETIC DEVICES}

Through the literature review developed in chapter 2, the different working principles developed in the research of thermomagnetic motors can be identified. To investigate the applicability potential of the most promising designs a thermodynamics analysis approach is proposed. This evaluation is possible only if adequate models are used to describe the magnetization work produced by a material moving into a magnetic field, and also adequate magnetic material data.

The working principle of a thermomagnetic device is based on the interaction between a magnetic material, that is being cyclically heated and cooled, and an applied magnetic field gradient. This interaction can be modeled following the classical approach proposed by Bozorth (1978), as shown in Figure 17.

Figure 17: The modeling of the interaction between a magnetic material and an applied magnetic field gradient.

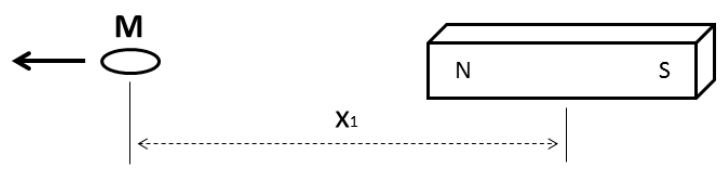

Source: Bozorth (1978)

From the model the specific force $(f)$ can be described by the following equation, also regarded as the Kelvin force equation (KITANOVSKI; EGOLF, 2006):

$$
f=\frac{\mu_{0}}{\rho} M \frac{\partial H}{\partial x}
$$

Where $f$ is the magnetically induced specific force in the material $(\mathrm{N} / \mathrm{kg}), \rho$ is the material density $\left(\mathrm{kg} / \mathrm{m}^{3}\right), M$ is the magnetization of the material $(\mathrm{A} / \mathrm{m}), H$ is the applied magnetic field strength $(\mathrm{A} / \mathrm{m})$, and $x(\mathrm{~m})$ is the distance between the magnetic material and the permanent magnet that generates the applied magnetic field gradient. It follows that the work produced by a magnetic material that is being moved inside an applied magnetic field gradient can be derived from equation 3.1. This magnetization work is defined by the magnetic co-energy $\left(w_{c}\right)$, which is defined by:

$$
w_{c}=\int_{0}^{H} B d H
$$


With $w_{c}$ being the energy density $\left(\mathrm{J} / \mathrm{m}^{3}\right)$ and $B$ the induced magnetic field $(\mathrm{T})$. The magnetization co-energy can then be integrated into a defined volume to calculate the total magnetization work. This magnetizing co-energy is also correlated to the magnetic field energy, which is more adequately related to the magnetic energy $\left(w_{m}\right.$, in $\left.\mathrm{J} / \mathrm{m}^{3}\right)$, and is defined by:

$$
w_{m}=\int_{0}^{B} H d B
$$

A graphical representation of the magnetic energy and co-energy densities is presented in Figure 18 to demonstrate the importance of these relations. In the figure the hatched areas represent the integrals from equations 3.2 and 3.3, these integrals are, in this case, calculated for the $B-H$ curve of a generic non-linear soft magnetic material. Both magnetic energy and co-energy are local quantities, that is, they are a function of the direction coordinates of the local applied field, and also depend on the operating point on the applicable $B$ - $H$ curve.

Figure 18: The correlation between the magnetic energy (red hatched area) and the magnetic co-energy (black hatched area) densities for a defined $B-H$ curve of a non-linear magnetic material.

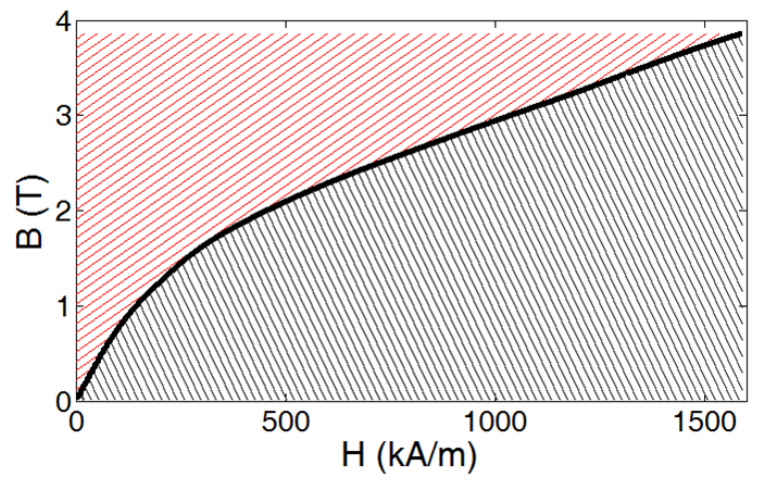

The behavior shown in Figure 18 is expected for any non-linear magnetic material, in which the co-energy density is expected to be greater than the magnetic energy density. The relevance of this analysis in the development of thermomagnetic devices is given by the fact that the magnetic co-energy will usually be greater than the magnetic energy, or in the least equal, for a given applied magnetic field. As the induced field change is dependent on the energy of magnetization, the devices that are based on the induced field change with the cyclically heating and cooling of a magnetic material, i.e., thermomagnetic generators like the pyromagnetic dynamo proposed by Edison (1892b) (Figure 3.b), will use a reduced part of the total energy product $\left(\mathrm{BH}_{\mathrm{MAX}}\right)$ of the applied magnetic field. 
On the other hand, the induced magnetic force and the magnetization work are dependent on the magnetic co-energy. Therefore, the devices that use the cyclically heating and cooling of magnetic materials to produce a magnetic force and with that obtaining mechanical work will use a higher part of $\mathrm{BH}_{\mathrm{MAX}}$., that is thermomagnetic motors like those initially proposed by Houston and Thomson (1879), Edison (1888), and Tesla (1889). From this analysis thermomagnetic motors seem to present a more interesting approach to the development of thermomagnetic devices, although it must be stated that the fact of having moving parts, and the conversion to mechanical work that must then be converted to some useful application, represent additional complications for such systems.

\subsection{Thermomagnetic Motors Models}

Through the literature review, two different approaches to the construction of thermomagnetic motors were identified. The first one is based on a continuous magnetic material that is heated and cooled simultaneously, producing a spatially static temperature gradient on the material and with that a magnetization gradient that is used to convert the heat into mechanical work. These systems are similar to the ones initially proposed by Houston and Thomson (1879) and by McGee (1884), and further developed by Edison (1888). This first approach will be referred as Edison type of thermomagnetic motor, although other names may be found on the literature, notably, as "Curie Wheel" or "Curie Motor" (ALVES et al., 2014; KARLE, 2001), these terms are not preferable as explained in chapter 2.

The second design approach to the development of thermomagnetic motors is based on the cyclical heating and cooling of a bulk material that is subjected to an applied magnetic field gradient, with the production of mechanical work and the heat transfer occurring in two separate moments of the motor cycle. This design is similar to the those proposed by Tesla (1889) and will be referred to as Tesla type of thermomagnetic motor.

To better understand the importance of the design differences between the Edison and Tesla type thermomagnetic motors, modeling of each design is proposed. For the Edison type of thermomagnetic motors, this modeling is based on the one proposed by Karle (2001) and is presented in Figure 19.a. The Edison type motor can be simplified from a disk or wheel into a continuous plate, as shown in Figure 19.b. In the figure, the letters marked present four 
distinct states in the cycle developed, the stages $\mathrm{A}$ and $\mathrm{D}$ indicate a region of zero magnetic fields where the magnetic material has the same temperature as the Heat Sink and Source, or more realistically the lowest and highest temperatures developed in a cycle. The stages B and $\mathrm{C}$ determine a region inside the applied magnetic field where the maximum field intensity is obtained, in a real application the location of these points is determined by the shape of the curve of the applied magnetic field intensity.

Figure 19: Design of the Edison type of Thermomagnetic Motor, in (a) a disk of magnetic material is used to produce a torque, and in (b) a simplified view of the design, focused on the work producing region.

Permanent magnet

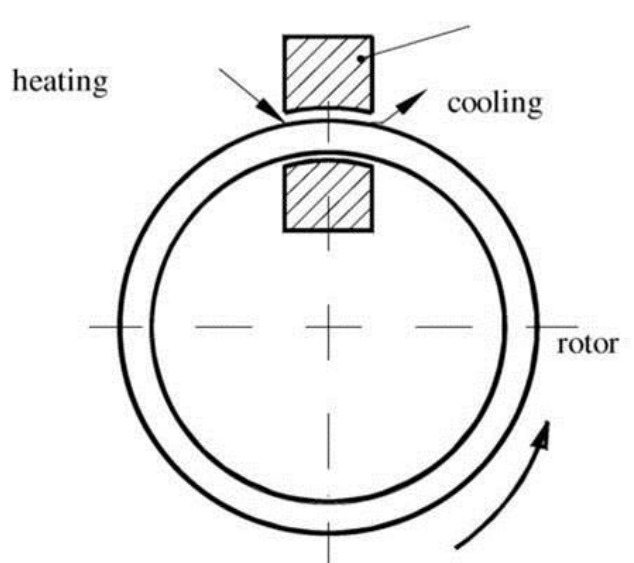

(a)

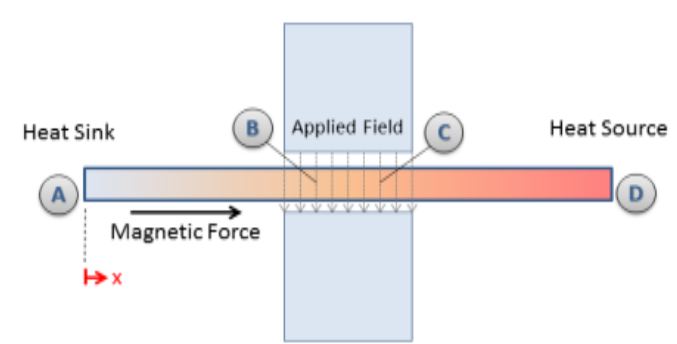

(b)

Source: Karle (2001) for (a), and own authorship for (b).

The thermodynamic cycle of the Edison type thermomagnetic motors can be described by estimating an applied magnetic field strength of $1.2 \mathrm{~T}$, and the temperatures of the heat source and heat sink are defined as 298 and $272 \mathrm{~K}$ respectively. With that, a graph indicating the state of the magnetic material as a function of its varying position is presented in Figure 20. In the figure, the position $\mathrm{X}$ is relative to the direction of movement from the zero position, as marked by the red arrow in Figure 19.b. 
Figure 20: The state of the magnetic material in an Edison type of Thermomagnetic Motor, based on the simplified view presented in Figure 19.b.

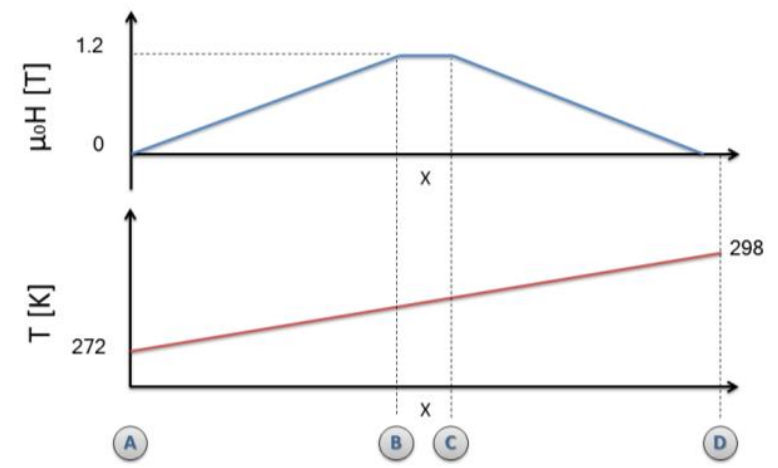

In Figure 20, the lower graph indicating the temperature of the material as a function of the position assumes a linear temperature increase from the heat sink to the heat source, this represents a material that ideally conducts heat between the two sources. In a real motor, this curve could be non-linear as the temperature change in the material causes the structural changes that promote the thermomagnetic effect will also cause changes in the specific heat of the material and its thermal conductivity. Another modifying factor is the movement of the material against the direction of heat conduction.

The model for the Tesla type thermomagnetic motors is presented in Figure 21. The model assumes the same temperatures of heat source and heat sink as the ones used for Edison type model (respectively 298 and $272 \mathrm{~K}$ ), and the same maximum applied magnetic field strength of $1.2 \mathrm{~T}$. The mechanical work is produced as the cold material is attracted into the higher magnetic field $(\mathrm{A} \rightarrow \mathrm{B})$, and an external mechanical power must be applied to remove the heated material from the higher intensity field $(C \rightarrow D)$. The heat transfer occurs in the constant field states, with the material being heated from $\mathrm{B} \rightarrow \mathrm{C}$ and cooled from $\mathrm{D} \rightarrow \mathrm{A}$. 
Figure 21: Simplified design of a Tesla type thermomagnetic motor.

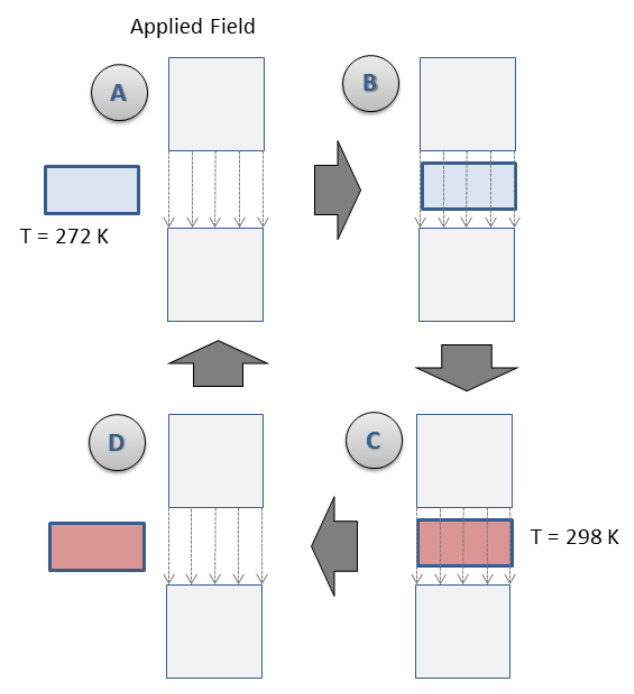

In the Tesla type motor there are two clearly defined states, the first one is A, where the material is in thermal equilibrium with the heat sink, and at a lower applied magnetic field strength, this lower value cannot be equal to zero, as a minimum applied magnetic field gradient is necessary to produce a starting magnetic force. The second defined stage is $\mathrm{C}$, where the material is in equilibrium with the heat source, and at the maximum applied magnetic field strength of 1.2 T. For the states B and D the applied magnetic field strength is determined, but two different conditions of temperature can be expected, with an isothermal magnetization process from $\mathrm{A} \rightarrow \mathrm{B}$ (and demagnetization in $\mathrm{C} \rightarrow \mathrm{D}$ ), or an adiabatic magnetization and demagnetization.

\subsection{Reference Magnetic Material}

A magnetic material must then be adopted to define the thermodynamic cycle developed by each of the models. Although many magnetic materials could be used, the material selected in this case will be Gadolinium $(\mathrm{Gd})$. This material is considered a reference material in the development of magnetocaloric and thermomagnetic devices (DAN'KOV et al., 1998), due to the fact of having a thermomagnetic phase transition near room temperature, with a considerable large magnetic moment, and being commercially available in its pure form. Gadolinium presents a second-order phase transformation, with no temperature hysteresis, and a critical transition temperature $\left(T_{C}\right)$ around $293 \mathrm{~K}$. 
The effect of the temperature increase in the magnetization of Gd can be described measuring the magnetization of the material as a function of an applied magnetic field, for different temperature conditions. The reduction in the magnetization for a given applied magnetic field as the temperature increases will be directly correlated to a reduction in the magnetic force induced on the material, according to equation 3.1, as can be observed in Figure 22, by tracing a vertical line at any applied magnetic field strength.

Figure 22: Magnetization of Gadolinium for a given applied magnetic field at different temperatures.

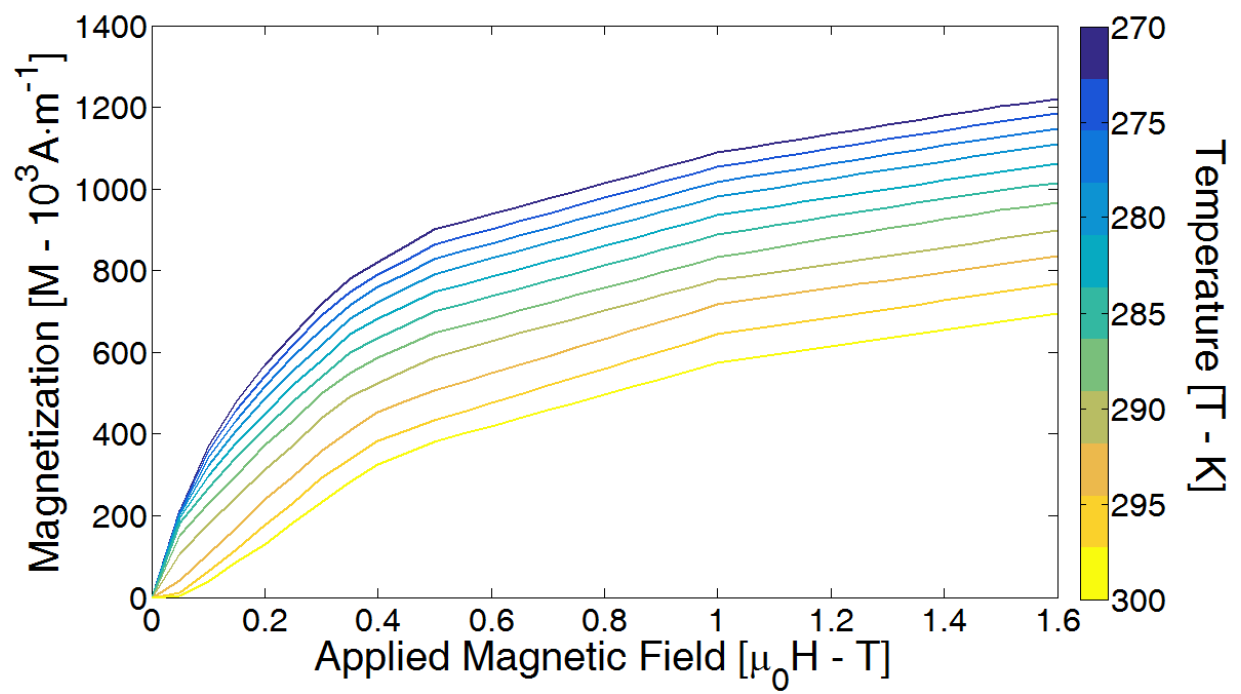

Source: Own authorship, with data from Risser et al. (2012).

A classical thermodynamic approach for the description of thermodynamic cycles is to use the temperature $\mathrm{x}$ specific entropy $(T-s)$, from which by describing a closed cycle the specific work can be calculated. The $T$-s curves for gadolinium will be influenced by the applied magnetic field strength, in an analogous manner to the effect of the pressure in the $T-s$ diagram for a fluid, this way the entropy is integrated from the temperature swapping specific heat measurements for different applied magnetic fields, with the resulting curves presented in Figure 23. 
Figure 23: The magnetic entropy change (T-s diagram) of Gadolinium.

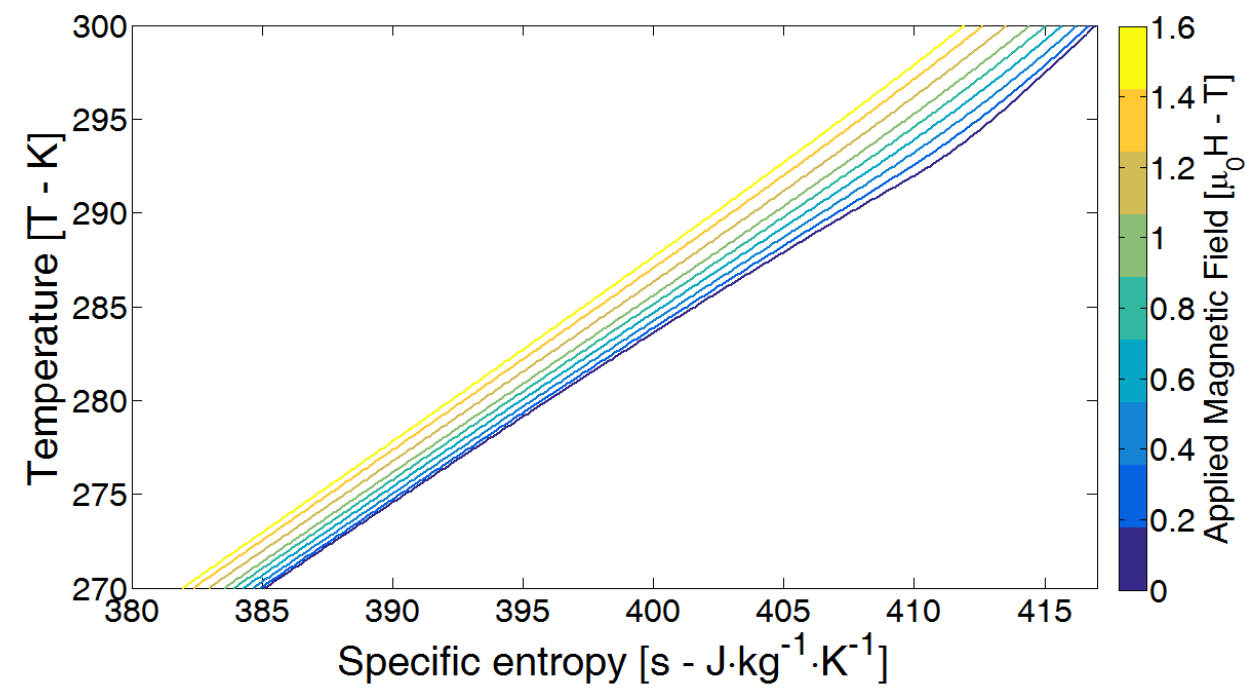

Source: Own authorship, with data from Risser et al. (2012, 2013).

\subsection{The thermodynamic cycle of thermomagnetic motors}

Using the diagrams presented in Figure 22 and Figure 23 the thermodynamic cycles developed by the modeled Edison and Tesla type thermomagnetic devices can finally be described. As in both models the temperatures of the heat source and sink are respectively 298 and $272 \mathrm{~K}$, the $T_{C}$ of the Gadolinium of $293 \mathrm{~K}$ will present a middle point between the heat sink and source, meaning that the thermomagnetic transition is occurring in each cycle of the motor, and with that obtaining the maximum magnetization variation in the material.

For the Edison type TMM, both temperature and applied magnetic field are changing with the movement of material, as shown in Figure 20, while heat is being transferred by the heat source and sink, and internally by conduction in the material. From the described material states the cycle developed by the Edison type TMM can be traced into the $M(H, T)$ diagram for Gadolinium, as presented in Figure 24. Initially, the material is in a zero applied magnetic field region and at a low temperature. In state A, the material is then moved into an increasingly higher field while its temperature is also increased by the conduction heat transfer $(\mathrm{A} \rightarrow \mathrm{B})$; the material will then reach a region of maximum applied magnetic field $(\mathrm{B})$ and continue to heat $(\mathrm{B} \rightarrow \mathrm{C})$, from which point the applied magnetic field strength will reduce continuously and the temperature increase until it reaches the maximum temperature and the applied field becomes zero $(\mathrm{C} \rightarrow \mathrm{D})$. The cycle is then closed as the material is cooled to the lowest temperature in a zero field region $(\mathrm{D} \rightarrow \mathrm{A})$. 
Figure 24: The cycle developed by the modeled Edison type thermomagnetic motor in the $M(H, T)$ diagram for Gadolinium.

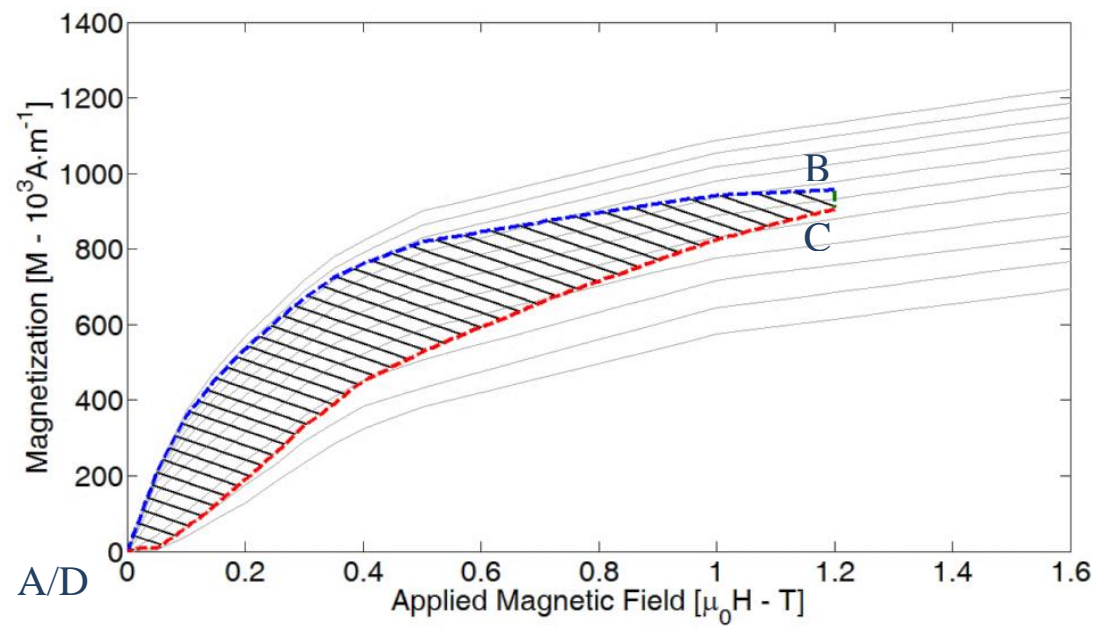

In Figure 24 the isothermal lines are presented in a grey color, becoming then clear that the processes $\mathrm{A} \rightarrow \mathrm{B}$ and $\mathrm{C} \rightarrow \mathrm{D}$ present an increase in the material temperature. In the model presented in Figure 20 a region of constant maximum field strength is proposed, which presents the process $\mathrm{B} \rightarrow \mathrm{C}$, this region could also be considered a single point of maximum applied magnetic field strength, in which case B and C would be superimposed. The points $\mathrm{A}$ and $\mathrm{D}$ are overlaid in the figure because the applied magnetic field strength in both cases is zero, and in which case the magnetization will be zero for all temperatures. It's important to notice that although the hatched area describes the motor cycle, it has no direct physical meaning, to describe thermodynamically the work done by the motor the $T(s, H)$ diagram must be used to trace the cycle of the Edison type TMM, as presented in Figure 25. 
Figure 25: The undefined thermodynamic developed by the modeled Edison type TMM in the T(s,H) diagram of Gadolinium.

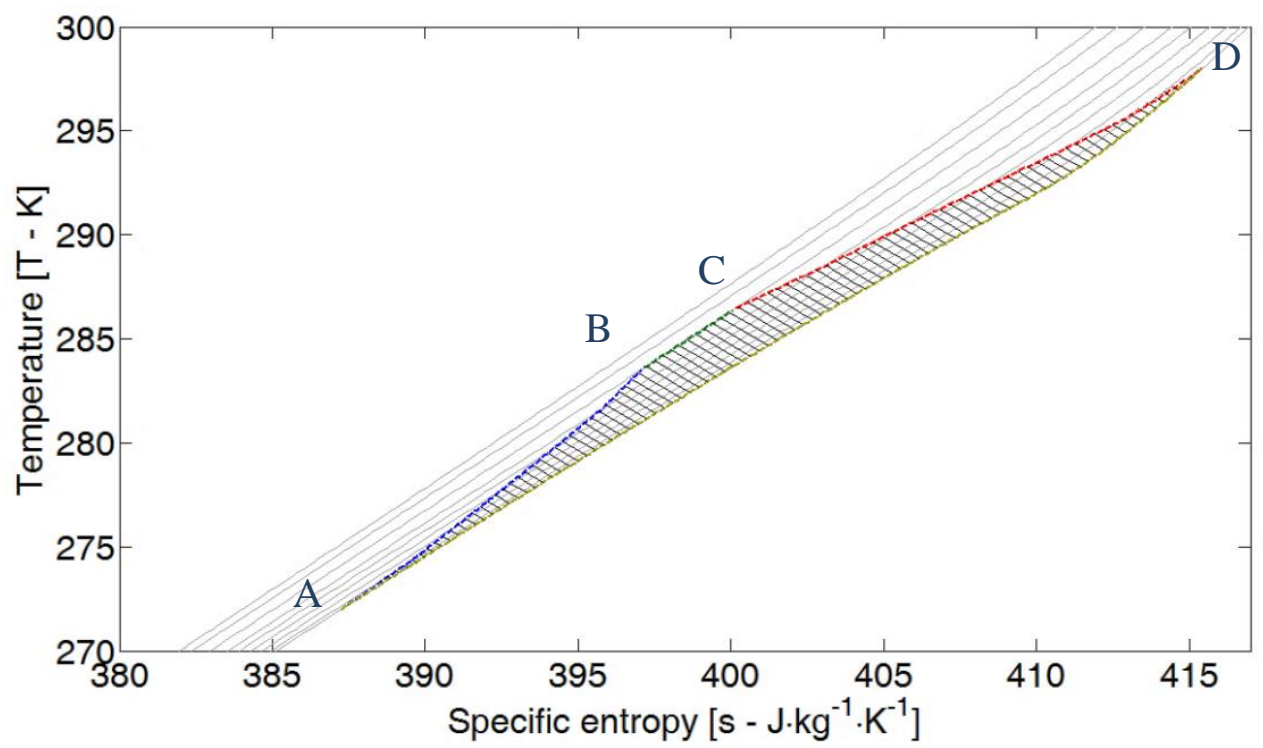

In Figure 25 the isofield lines are presented in a grey color, from which can be noted that the applied magnetic field strength is increasing from $\mathrm{A} \rightarrow \mathrm{B}$. It remains constant at the maximum applied magnetic field while the material continues to heat and therefore briefly follows a constant applied magnetic field from $\mathrm{B} \rightarrow \mathrm{C}$, and decreases continuously from $\mathrm{C} \rightarrow \mathrm{D}$. The cycle is then closed as the material is cooled in a constant zero field line from $\mathrm{D} \rightarrow \mathrm{A}$. By integrating the cycle described by the motor, presented by the hatched area in the graph, the specific work per cycle is calculated to be $41.08 \mathrm{~J} \cdot \mathrm{kg}^{-1}$.

One important point to notice is that, according to the model of the Edison type TMM presented in Figure 20, during the cycle the material is being constantly heated from $A \rightarrow D$ and then cooled in $\mathrm{D} \rightarrow \mathrm{A}$, presenting constant applied magnetic fields in the regions described by $\mathrm{B} \rightarrow \mathrm{C}$ and $\mathrm{D} \rightarrow \mathrm{A}$, and from the cycle described in Figure 25 the motor does not present any isothermal or adiabatic process. This means that no equilibrium process can be clearly defined for the modeled Edison type of TMM, and that the thermodynamic cycle for such motors cannot be easily identified as any classical thermodynamic cycle.

Although it was previously proposed to use a Brayton cycle to calculate the efficiency of Edison type TMM (EGOLF et al., 2009), this cycle would most definitely not represent a realistic Edison motor. As this type of TMM depend on the heat transfer inside the material through conduction, and the classic Brayton cycle proposes an adiabatic pressure change, which could be interpreted as an adiabatic change in the applied field to the material. 
For the Tesla type thermomagnetic motors, the model presented in Figure 21 presents the clearly defined stages A and C, and as previously explained, the conditions B and D will depend on the assumption of how the magnetization change in the material occurs, in either an isothermal or an adiabatic manner. This consideration is important due to the magnetocaloric effect in the material, i.e., the reversible heating or cooling of a magnetic material as it is adiabatically magnetized or demagnetized. The influence of such consideration can be demonstrated through the $M(H, T)$ diagram of Gadolinium presented in Figure 26, in it the isothermal magnetization lines are presented in grey and the defined points $\mathrm{A}$ (with the material cooled and at a lower magnetic field) and $\mathrm{C}$ (with the material heated and at a higher magnetic field) marked. The dotted lines represent an isothermal change in magnetization, and the solid lines present the adiabatic change.

Figure 26: From the defined thermodynamic states A and C, two possible behaviors can be described by a Tesla type TMM, an isothermal (dotted lines) or an adiabatic (solid lines) magnetization/demagnetization.

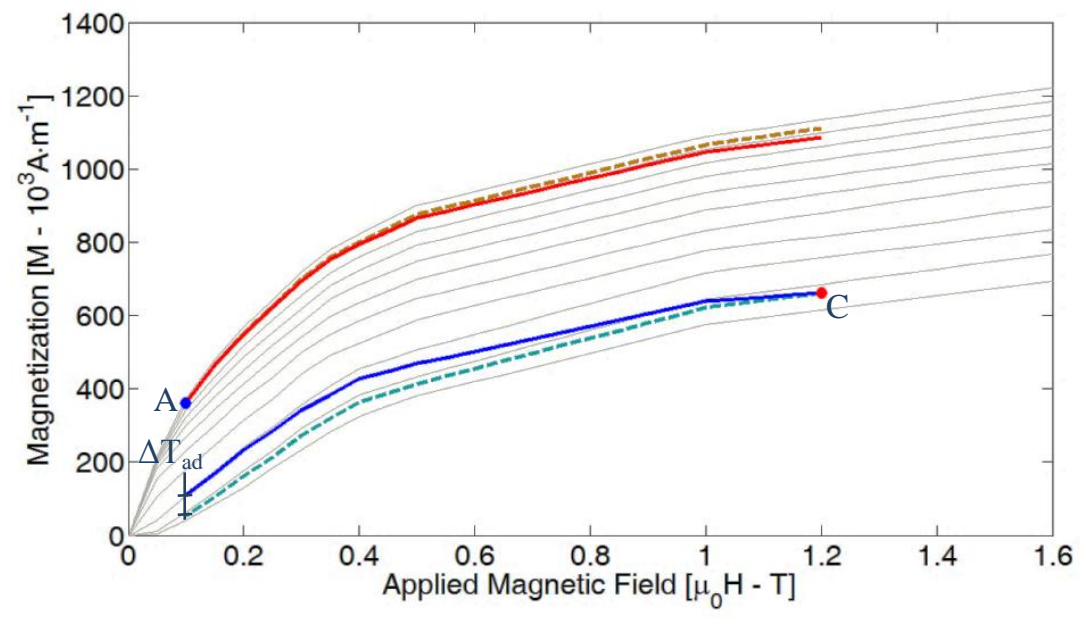

From the curves shown in Figure 26, the adiabatic temperature change in the material $\left(\Delta T_{A D}\right)$ due to the magnetization process in the points $\mathrm{B}$ and $\mathrm{D}$ can be observed, the magnitude of such change is presented in the cycle of a Tesla type TMM for the $T(s, H)$ diagram. Due to the dynamics, the Tesla type motors, in which the reversible magnetization process is very rapid during the work production part of the cycle, the adiabatic process can be assumed. With that and the described material states, a cycle for the Tesla type TMM can be traced into the $M(H, T)$ diagram for the Gadolinium material, as presented in Figure 27. 
Figure 27: The cycle developed by the Tesla type thermomagnetic motor in the $M(H, T)$ diagram for Gadolinium, assuming an adiabatic magnetization/demagnetization process, with the effect of the magnetocaloric effect.

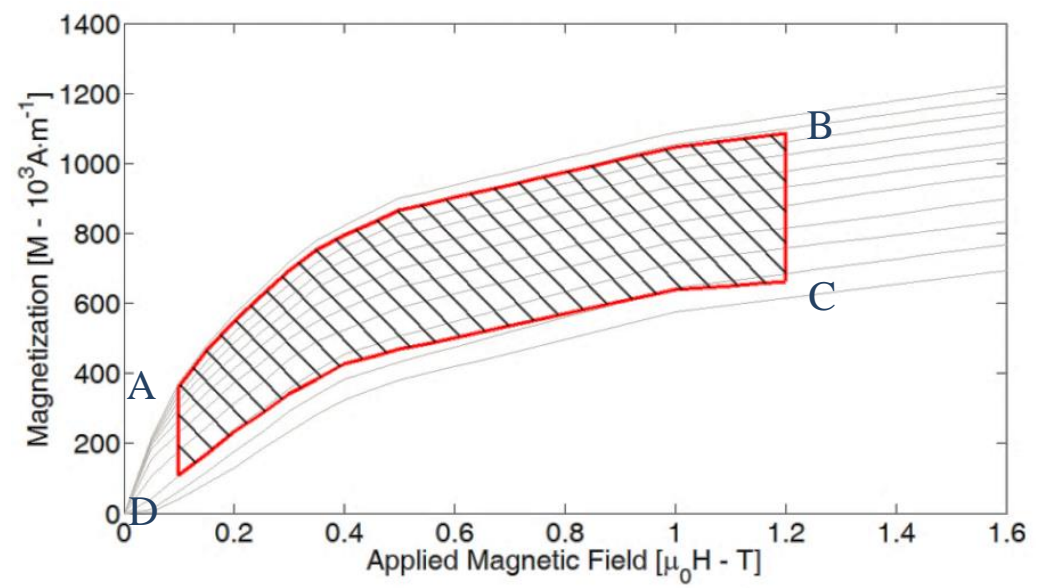

In Figure 27, the initial state A occurs right before the movement of the material, with the material in equilibrium with the heat sink at $272 \mathrm{~K}$. Then, as the material moves, it is adiabatically magnetized in $\mathrm{A} \rightarrow \mathrm{B}$, heated by a small $\Delta T_{a d}$, to state $\mathrm{B}$. Inside the high applied magnetic field region the material is heated to the temperature of the heat source of $298 \mathrm{~K}$ $(\mathrm{B} \rightarrow \mathrm{C})$, being then adiabatically removed from the magnetic field by the action of an external force $(\mathrm{C} \rightarrow \mathrm{D})$, and its temperature reduced by a small $\Delta T_{a d}$ due to the magnetocaloric effect. In the state D the material is cooled and the cycle restarted. During the cooling part of the cycle $(\mathrm{D} \rightarrow \mathrm{A})$ the material is at the lowest applied magnetic field, which for the Tesla type TMM cannot be considered zero, as some minimum applied magnetic field gradient is necessary to start the movement of the material, in the model a minimum applied magnetic field strength of $0.1 \mathrm{~T}$ is adopted.

To describe the thermodynamic cycle of the modeled Tesla type TMM the cycle is traced into the $T(s, H)$ diagram for $\mathrm{Gd}$, as presented in Figure 28. In the figure the isofield lines are presented in grey, from it can be seen that both the heating $(B \rightarrow C)$ and cooling $(\mathrm{D} \rightarrow A)$ processes occur in a constant field. In the graph, the red encircled area presents the assumption of adiabatic magnetic field changes. The resulting temperature change $\left(\Delta T_{a d}\right)$ is a function of the applied magnetic field change and the temperature of the material, for the magnetization $(\mathrm{A} \rightarrow \mathrm{B})$. The observed $\Delta T_{a d}$ increase is of $2 \mathrm{~K}$, and for the demagnetization the $\Delta T_{a d}$ reduction is of $3.1 \mathrm{~K}$. By integrating the cycle, presented by the hatched area in the figure, the specific work for Tesla type of TMM for each cycle is calculated to be $67.23 \mathrm{~J} \cdot \mathrm{kg}^{-1}$. 
Figure 28: The thermodynamic cycle developed by the Tesla type TMM in the $T(s, H)$ diagram of Gadolinium, the red encircled area defines a magnetic equivalent Brayton cycle.

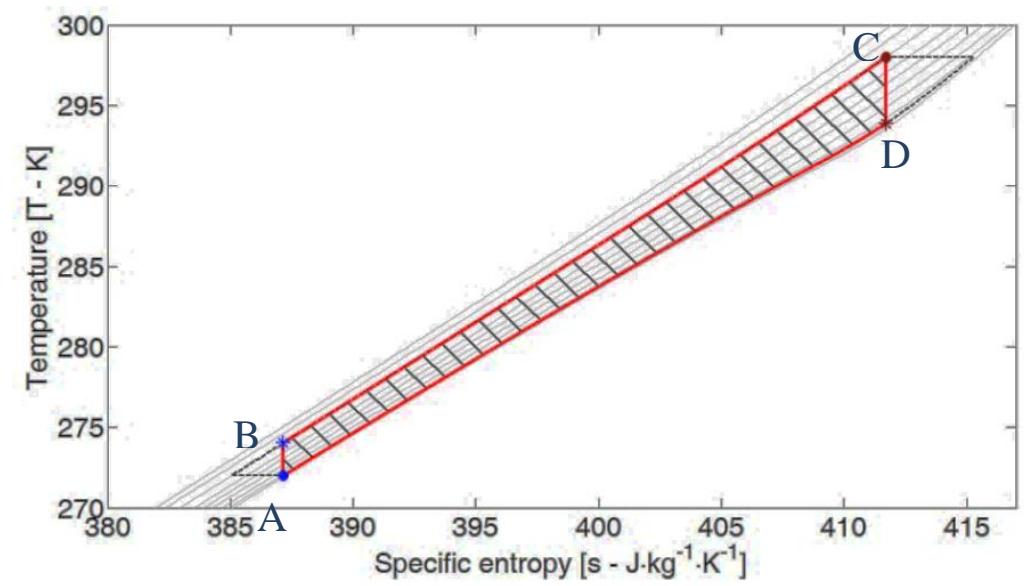

In Figure 28, the dotted lines present an assumption of an isothermal change in the applied magnetic field in the material, with that the area described by the graph increases, indicating an increase in the specific work per cycle of the motor. But this increase also indicate a reduction in the efficiency of the system modeled, since more heat is required to bring the motor from $\mathrm{A}$ to the state $\mathrm{B}$. The adiabatic changes in the magnetization of the material can for the Tesla type motor be correlated to adiabatic pressure change in a classic Brayton cycle, in this case applied magnetic field will be analogous to the pressure change in a fluid, and with that the Tesla type thermomagnetic motors can adequately be modeled by an adapted magnetic Brayton cycle.

At this point, an interesting comparison can be made between the Edison and Tesla types of thermomagnetic motors from a thermodynamic perspective. Based on the previous analysis and calculation of the specific work per cycle the Tesla type TMM will produce a greater amount of work, with a percentage increase of $63.7 \%$. This while presenting the same maximum applied magnetic field strength of $1.2 \mathrm{~T}$ and the same temperatures of heat source and sink. To analyze the difference in efficiency between these two types of motors the second order efficiency must be considered.

The energy quality concept represents the capacity of a given source to cause change, as characterized by its entropy level and depending on both the form of energy (chemical, thermal, etc.) and on the parameters of the energy carriers and that of the surrounding environment. As defined by Kotas (1985), to account for the variable quality of different disordered energy forms in the analysis of thermal plants, the natural standard to be 
established is that of the maximum work which can be obtained from a given form of energy using the environmental parameters as the reference state (surrounding atmospheric pressure $-P_{0}$, temperature $-T_{0}$, and concentrations $-c_{0}$ ). This standard energy quality defines the concept of exergy. The exergetic efficiency, in this case, represents relative efficiency between a real process and the idealized reversible process, which is defined by the Carnot efficiency, this way:

$$
\eta_{\text {Exe }}=\frac{\eta_{\text {Real }}}{\eta_{\text {Carnot }}}
$$

Where $\eta_{\text {Carnot }}$ presents the maximum efficiency available for a given temperature difference, operating in a reversible cycle, with:

$$
\eta_{\text {Carnot }}=1-\frac{T_{\text {cold }}}{T_{\text {hot }}}
$$

Using equation 3.6 the maximum efficiency for any cycle operating in the temperatures used to model the Edison and Tesla type thermomagnetic motors, with $T_{\text {cold }}=272 \mathrm{~K}$ and $T_{h o t}=298 \mathrm{~K}$, will be of $8.7 \%$. The efficiencies of the motors modeled are calculated by integrating the cycle developed by the motor and obtaining their specific work, and then dividing it by the total amount of energy expended in the heating process, obtained by integrating the heating process for the Edison $(A \rightarrow B \rightarrow C \rightarrow D)$ and Tesla type $(B \rightarrow C)$. With that the second order efficiencies can be obtained using equation 3.5, the term "exergetic efficiency" is not applied in this sense since the reference state adopted $\left(T_{\text {cold }}\right)$ is not the standard environmental parameter $\left(T_{0}=298 \mathrm{~K}\right)$. For the systems modeled the second order efficiency for an Edison type TMM will be of 5.84\% and for the Tesla type of $11 \%$.

Based on the models developed and on the performance calculations for the Edison and Tesla types Thermomagnetic motors, it can be concluded that the Tesla type motor will present the better concept. Assuming the same conditions of maximum applied magnetic field strength and temperatures, it was shown that Tesla type of design produces a higher amount of work for the same mass of magnetic material and obtains a higher efficiency. Although the values obtained in the calculations do not take into account the uncertainties in the material data, and the resulting uncertainty in the calculations, with the used approach limited to a qualitative result. The differences observed are high enough to indicate that the tendency obtained can be expected for more realistic calculations and models. 


\section{THE THERMOMAGNETIC MOTORS DEVELOPED}

This chapter presents the design and basic characteristics of the two devices built to study the basic principles of thermomagnetic motors, and with that develop the technological concept to a laboratory scale demonstrator, allowing the system validation in both experimental and simulation levels. As shown in chapter 1, the Tesla type thermomagnetic motor presents a high potential to be used in the conversion of low-grade heat energy into useful mechanical energy, being the design of TMM adopted for development in this thesis.

\subsection{The idealized Tesla type Thermomagnetic Motor}

As previously demonstrated a thermomagnetic motor operating with heat sources close to room temperature will necessarily operate with low energetic efficiency, due to the limiting Carnot efficiency in this temperature range. Because of that, any experimental system that is operated with a low power input will obtain exceedingly low power outputs. This implies in an elevated influence of any external losses that may be caused by: friction in the moving parts; heat transfer irreversibilities; the influence of the applied magnetic field, either by the interaction with ferromagnetic materials that may be part of the motor or by eddy currents induced in any electrically conductive material; and any misalignments or mounting adjustments. These losses, which are usually negligible in conventional heat engines, can be expected to have the same magnitude as the maximal theoretical output for a thermomagnetic motor operating in these relatively low heat source temperatures $(340-355 \mathrm{~K})$.

One possible solution, as to allow the measurable power outputs, is to build bigger experimental systems. By increasing the input heat flow in the motor, and with that utilizing larger magnetic material masses and higher applied magnetic fields, the relative influence of the losses in the system is expected to reduce. This way, even a first step experimental validation of the theoretical power output of thermomagnetic motors would demand the construction of a prototype of large dimensions and increased complexity.

An alternative solution can be the construction of a test bench capable of representing the functionality of a thermomagnetic motor in an idealized manner. In essence, this test stand must be capable of measuring the magnetic force produced by the interaction of the 
magnetic field gradient produced by a permanent magnets assembly and a magnetic material at varying distances and temperatures. The resulting curve can then be used to calculate the available magnetic work presented in a thermomagnetic motor operating between two measured temperatures and with a given displacement. This represents the magnetic work of an idealized motor built with the measured magnetic material and working with the applied field gradient produced by the permanent magnets assembly.

This system must, therefore, be capable of accounting for any external losses that would be expected in a real motor, removing the friction influence and having precise control of the temperature of the magnetic material while measuring the magnetic force induced by a known applied magnetic field gradient. This serves two practical purposes: the first is the validation of the theoretical power output expected in the motor and of its efficiency; and the second purpose is to allow an experimental comparison of different materials that could be applied in the construction of thermomagnetic motors, regarding their maximal power output. The proposed test stand of an idealized thermomagnetic motor is presented in Figure 29.

Figure 29: A schematic view of the proposed test stand to measure the magnetization work of magnetic materials (a), with a section view of the sample holder (b).

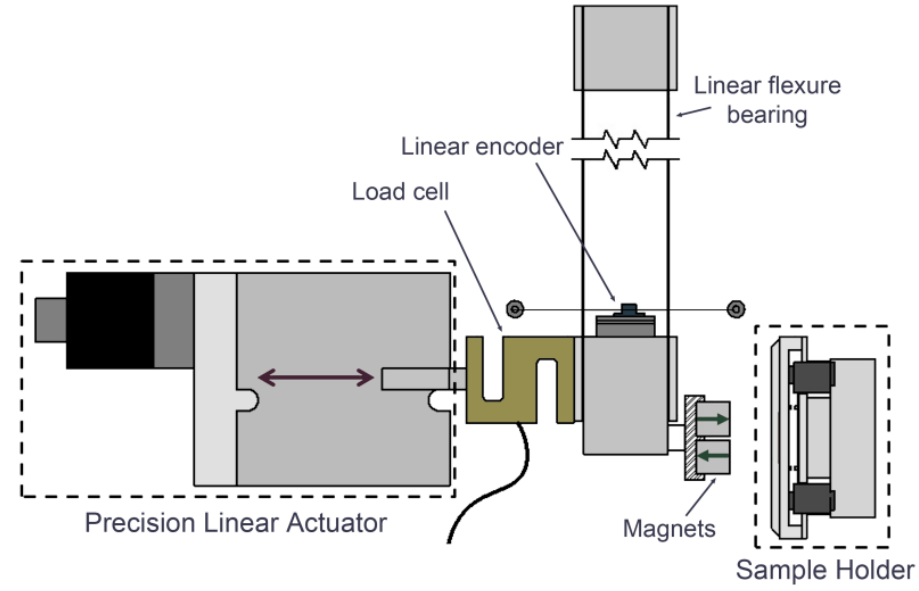

(a)

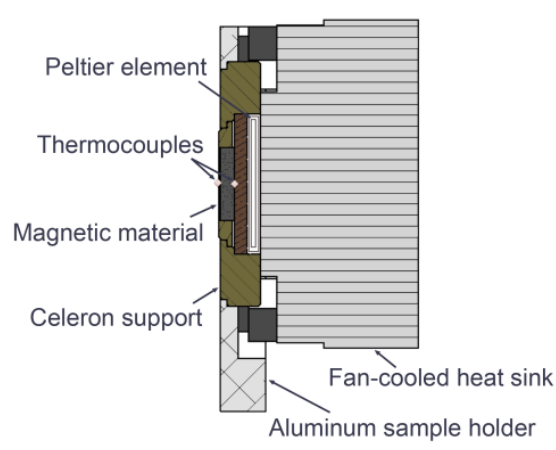

(b)

A linear flexure bearing is used to avoid the influence of the friction in the measurement, which presents a random uncertainty due to the stick-slip effect,. This type of support uses an arrangement of flexure elements held by rectangular mounting blocks, while one of the blocks is fixed in place (top of Figure 29.a) the other is free to move, being compliant in a single degree of freedom, in the proposed design only in the direction of the sample. The use of the flexure bearing imposes a spring resistance to the motion that is 
predictable and can be calibrated within a known uncertainty. The plates to be used in the design of the linear flexure bearing are fabricated of non-magnetic AISI 316 stainless steel, and have a length of $600 \mathrm{~mm}$, providing a low spring action in the range of motion of the experiment.

A precision linear actuator is used to position the magnets assembly in relation to the fixed sample holder. The movement of this actuator is done by a ball screw shaft actuated by a stepper motor. Initial tests showed this precision actuator is capable of producing repeatable displacements inferior to $10 \mu \mathrm{m} \pm 3 \mu \mathrm{m}$ (measured with the use of a machinist dial indicator with a minimum resolution of $1 \mu \mathrm{m})$.

The load cell used to measure the magnetic force in the system is mounted between the linear actuator and the linear flexure bearing, this way any force present in the system is measured, including the spring resistance of the flexure bearing which is then subtracted in the data acquisition system. An Alfa S-5 load cell is used (ALFA INSTRUMENTOS, 2006), which has a nominal capacity of $50 \mathrm{~N}$, is made of aluminum, and according to the manufacturer's brochure has a sensibility of $2.0 \pm 10 \% \mathrm{mV} / \mathrm{V}$ with temperature compensation up to $323 \mathrm{~K}$. As this load cell was available in the laboratory from a previous experiment, and had to be integrated into the data acquisition system (DAQ), a careful process of recalibration and verification was conducted using standard masses, which showed that the nominal sensitivity of $0.01 \mathrm{~N}$ was achieved, with an uncertainty of $\pm 0.05 \mathrm{~N}$ mainly provided by the DAQ signal noise.

Because of the way the load cell is mounted, its deformation will influence the actual positioning of the flexure bearing, while this uncertainty may be minimal, it is a difficult one to account for during the experimental procedure. Therefore, to obtain the true position of the system, a linear encoder is mounted to the flexure bearing moving head, as shown in Figure 29 , allowing the measurement of the correct relative position between the magnets assembly and the sample holder. The incremental linear encoder used in the system has a resolution of $70 \pm 20 \mu \mathrm{m}$ when using the double precision reading $(2 \mathrm{X})$. The position zero was assumed for the position in which the magnets assembly touches the sample holder.

The initially adopted geometry of the sample of magnetic material analyzed in the system has a coin shape with a diameter of $15 \mathrm{~mm}$, and the mounting system can be adapted for different thicknesses. In the sample holder, shown in Figure 29.b, a Celeron support 
compresses the magnetic material to one of the faces of a Peltier element, used as a cooler/heat pump to control the temperature of the sample, the Celeron support also functions as thermal insulation between the magnetic material and the surrounding environment.

The Peltier element used in the system is a model TEC1-12706 (THERMONAMIC MODULE, 2017), with a thermal contact area of $40 \times 40 \mathrm{~mm}$ and a thickness of $3.8 \mathrm{~mm}$. This Peltier element is rated for up to $12 \mathrm{~V}$ with a cooling capacity of up to $60 \mathrm{~W}$. The air-cooled heat sink mounted in contact with the "hot face" of the Peltier element disperses the pumped heat to the environment, a computer cooler model Intel Socket 1155 was used in the system, which allowed the sample to be cooled to a temperature of $278 \mathrm{~K}$. By controlling the voltage applied to the Peltier element, it is possible to control the cooling power applied and with that the temperature of the sample, if the applied voltage is reversed then the Peltier element actuates as a heat pump, heating the magnetic material to $373 \mathrm{~K}$, limited by the rated maximum temperature of the Peltier used.

Two N-type thermocouples are mounted to the faces of the sample analyzed, the mean temperature of the sample then acts as process variable for a calibrated PID controller, that adjusts the applied voltage intensity and direction in the Peltier element used according to the specified setpoint. In the built system this controller was found to be able to stably adjust the mean temperature of the sample within $1.0 \pm 0.5 \mathrm{~K}$, having a higher uncertainty in the temperatures close to room temperature, situation in which the control variable gets closer to zero.

The built test stand is presented in Figure 30, the base in which the system is mounted and the sample holder structures are made of aluminum. Care was taken to avoid the influence of any external ferromagnetic material in the measured force. Also, to avoid the influence of eddy currents in the system, the measurements are realized statically, with the evaluated force only being registered with the system stopped and after the load cell present a stable measurement. 
Figure 30: Pictures of the test stand to measure the magnetic force of a magnets assembly over a magnetic material with controlled temperature.

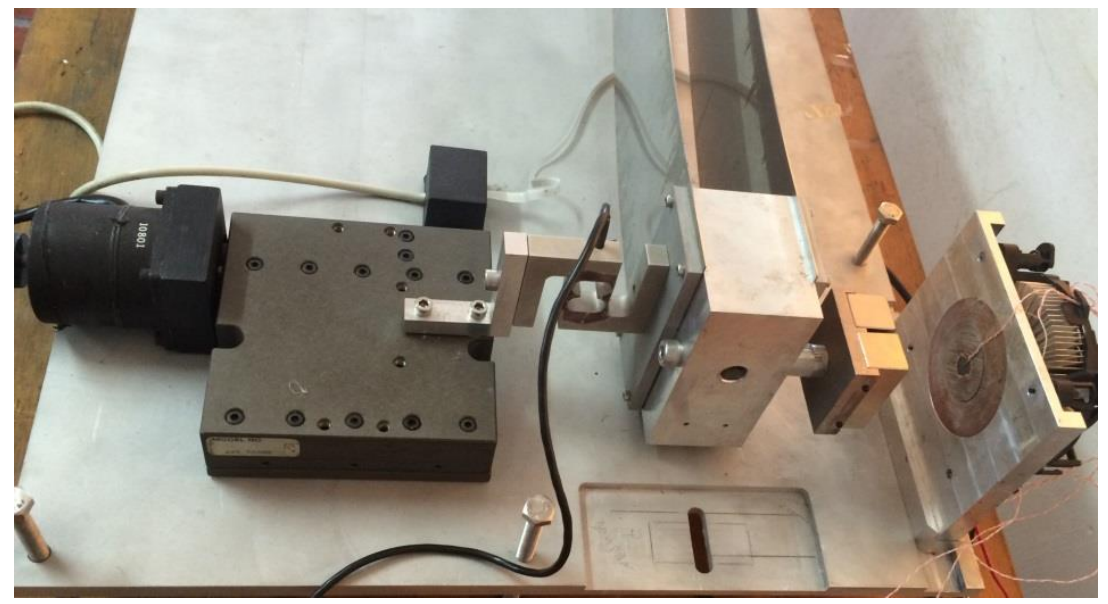

(a)

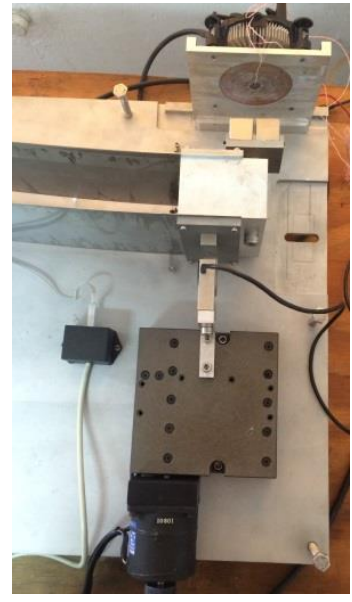

(b)

\subsection{The Rotary Thermomagnetic Motor}

The Tesla type thermomagnetic motors present a promising design for thermomagnetic devices. As shown in chapter 3, it can potentially produce a higher amount of work per kilogram of magnetic material and a higher efficiency than the Edison type thermomagnetic devices. Despite this potential, the design proposed by Tesla presents one fundamental challenge, which is the nature of the reciprocating movement developed by the magnetic material, as presented in the modeling of the Tesla type TMM in Figure 21. While the mechanical work produced in each stroke of the motor can be directly used in some desired process, e.g., in the compression of a gas, the conversion of the linear movement into a practical axis rotation presents a challenge.

The advantage of having a rotary movement was already devised by Tesla in his initial patent (TESLA, 1889), in which he proposes the use of a slide-crank mechanism connected to a flywheel, as shown in Figure 31. The TMM presented in the figure works with the same principles as the one shown in Figure 4. Additionally, the flywheel is supposed to provide the action of removing the heated material always from the magnetic field, acting in the place of the spring used in the model shown in Figure 4. 
Figure 31: The use of a slide-crank mechanism to convert the reciprocating movement of a TMM into a rotary movement, with the flywheel also acting to remove the material from the heat source.

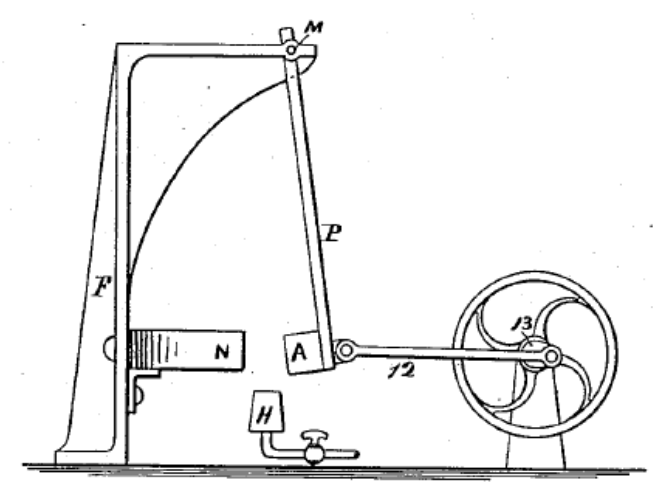

Source: Tesla (1889).

The main challenge in using such slider-crank mechanism is presented by the different dynamics of the magnetic action, which acts to rapidly attract the material, and the heat transfer, which is commonly a slower process. This difference could cause the mechanism to be locked in position as the flywheel slows. One possibility to address this challenge is to design the thermomagnetic motor to produce a rotary movement, working in a step manner, similarly to an electric stepper motor. This solution was proposed by Merkl (1969), in a patent titled "Step-by-step Thermo-magnetic motor," whose main design is presented in Figure 32. In it, 12 magnetic material parts are alternately heated and cooled, with an inner rotor that is rotated by the action of the magnetic force induced by four permanent magnets. As the rotor moves, the heat is commutated to produce a continuous rotation movement.

Figure 32: The working diagram of the "Step-by-step Thermo-magnetic motor," working in the same principle as the Tesla type TMM.

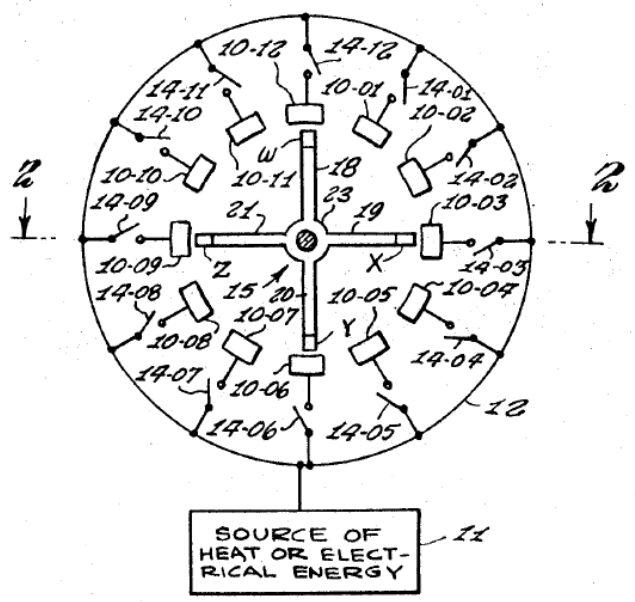

Source: Merkl (1969). 
The thermomagnetic motor proposed by Merkl (1969) works in the same principle as the Tesla type TMM, in the sense that each magnetic material part is heated or cooled in bulk, with no heat transfer between the parts. As the magnets can freely rotate in the direction of cooled materials, the movement is supposedly fast, in a way that can also admit the assumption of an adiabatic change in the magnetization of the material. In this patent, the magnetic materials are heated by the action of the electrical current, or another unspecified heat source, and cooled by the heat transfer with the environment.

A rotary Tesla type thermomagnetic motor was then proposed by Coelho, Gama and Silva (2012), in a patent titled "Dispositivo Rotativo Termomagnético e Uso do Mesmo" (Rotary Thermomagnetic Device and its use). This system is focused on the conversion of the heat from low-grade sources (with temperatures below $373 \mathrm{~K}$ ), and working with heat transfer fluids for the heating and cooling processes of a magnetic material that has a $T_{C}$ close to room temperature. The working scheme of this device is presented in Figure 33, where three magnetic material plates (M1, M2, and M3) are fixed to an external structure and act as the stator of the motor. Two magnets assembly are fixed to a rotary axis and act as the rotor, and three sensors (S1, S2, and S3) are used to determine the position of the rotor and control the heating and cooling of the plates accordingly.

Figure 33: Working scheme of the rotary thermomagnetic motor proposed by Coelho, Gama and Silva (2012), with a step rotation of $60^{\circ}$ between each stage and three magnetic material plates.

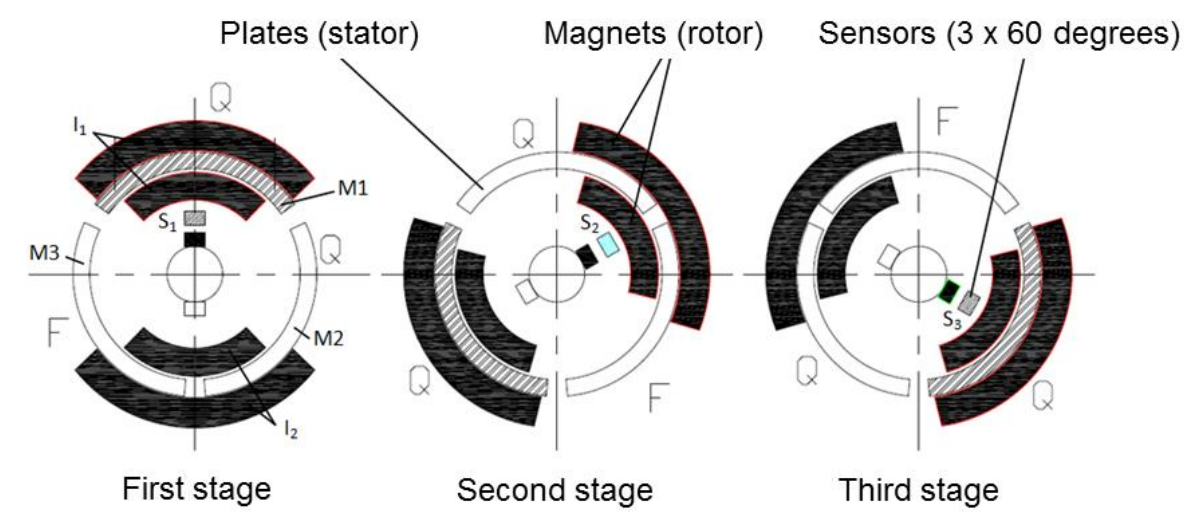

Source: Adapted from Coelho, Gama and Silva (2012).

The plates of magnetic material in the design shown in Figure 33 have internal channels for the flow of heat transfer fluids that can act both for the heating and cooling of the material. The cycle of the motor proposed then occurs in three separate stages. In the first stage, plates M1 and M2 receive heat from the heat source, while the flow of fluid from a 
heat sink cools plate M3. As M1 and M2 temperatures increase their magnetizations decrease by the thermomagnetic effect. At this point the magnetic force produced by the interaction of the magnets assembly I2 with the cooled plate M3 causes the rotor to produce a rotary step of $60^{\circ}$, taking the system to the second stage. At this point the sensor S2 detects the change in the position of the rotor, and commands a change in the valves controlling the flow of the heat transfer fluids, heating plates M1 and M3, while cooling the plate M2, once more producing an unbalanced magnetic force and taking the motor to the third stage. As a result, the sensor S3 commands the flow of fluids to cool the plate M1 and heat M2 and M3, until the rotary movement occurs and the cycle of the motor is reinitiated, with the magnets assembly I1 now in place of I2.

The rotary thermomagnetic motor proposed has its mechanical design presented in Figure 34. In the figure the motor stator supporting the magnetic plates (2) is fixed to the base of the motor (1), this base also supports the rotor axis of the motor (4). Support (3) is used to mount the magnets assembly to the rotor axis, and three proximity sensors are used to determine the angular positon of the rotor (5) and with that act to control the flow of the heat transfer fluids. Although this design has the advantage of directly producing rotary movement, while using the Tesla type TMM approach, the axis rotation will not be continuous, due to the different dynamics involved in the processes of magnetization work and heat transfer. To resolve this problem, Coelho, Gama and Silva (2012) suggested the use of a ratchet mechanism (6) to couple the intermittent axis rotation in the TMM to a flywheel (7), with the intent to smooth the power output and produce a continuous rotation.

Figure 34: Mechanical design of the rotary type thermomagnetic motor.

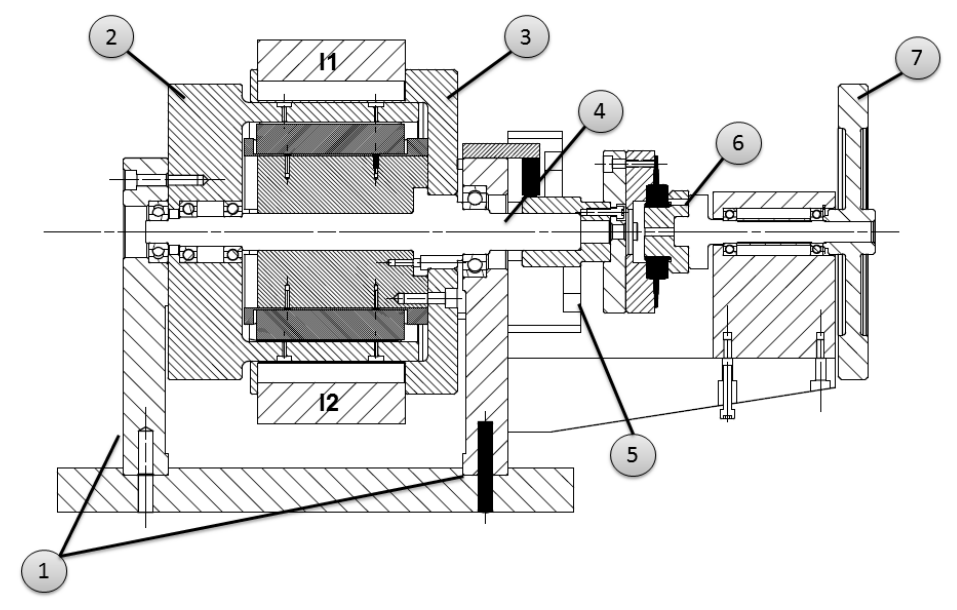

Source: Adapted from Coelho; Gama and Silva (2012). 
This rotary Tesla type thermomagnetic motor is then developed as a laboratory scale technology demonstrator. This device aims to model and validate the functionality of TMM in the application of the conversion of low-grade heat sources into mechanical energy, following the objective of advancing the technological readiness level of these machines. Initially, the mechanical construction was modeled using the software Autodesk Inventor, with the developed 3D model presented in Figure 35.

Figure 35: Modeling of the rotary Tesla type of thermomagnetic motor developed, in (a) a view of the system assembly and in (b) an exploded view showing the magnets assembly and the way the magnetic plates are fixed to the stator.

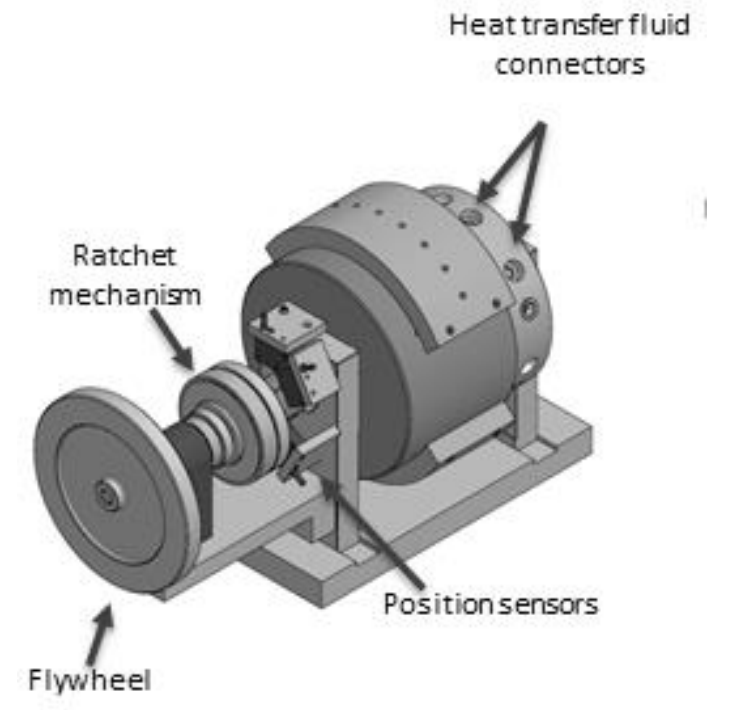

(a)

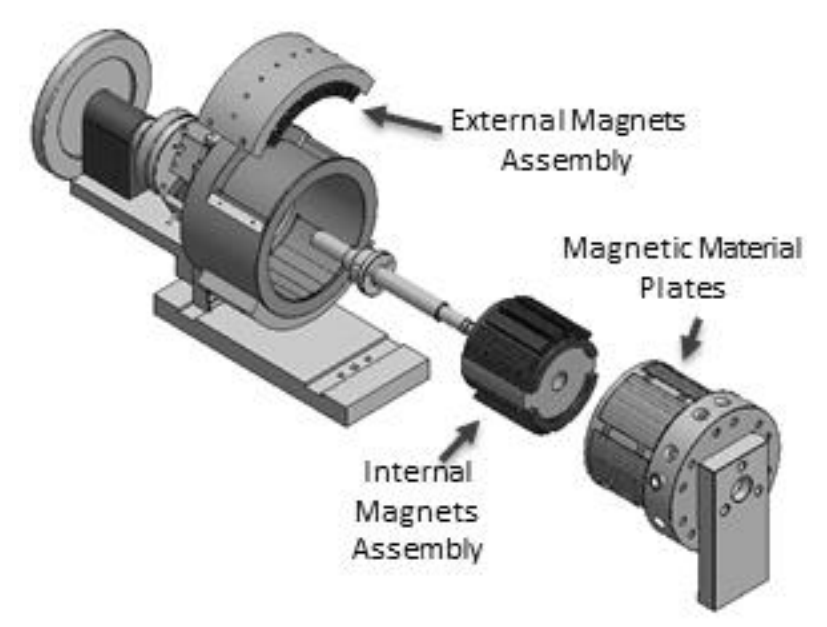

(b)

The model shown in Figure 35 follows the mechanical design presented in Figure 34, with the main parts indicated in the figure. In the design modeled the support of the magnetic material, plates act both the motor stator and as a fluid distribution connector to the magnetic material plates, which present internal channels for the flow of the heat transfer fluids. This design uses six magnetic material plates; instead of three as the originally patented design shown in Figure 33, these plates can be heated or cooled in pairs to present the same working cycle as the original design and also allow the possibility of exploring different working cycles.

The magnetic material to be used in thermomagnetic motors must be selected in function of the temperatures of the heat source and sink. Ideally, the critical transition temperature of the material $\left(T_{C}\right)$ must be below the temperature available from the source and above the temperature of the sink, with that, the maximum magnetization change in the 
material from the ferromagnetic-paramagnetic transition will occur in every cycle of the motor. As the aim of the technology demonstrator is to convert the heat from sources with temperatures in the range of 343 to $353 \mathrm{~K}$, and having a heat sink at room temperature $\left(T_{0}=298.15 \mathrm{~K}\right)$, the Gadolinium material used to model the thermodynamic behavior of TMM in chapter 3 which presents a $T_{C}$ of $293 \mathrm{~K}$ would not be a viable option.

Although the research of magnetic materials for energy conversion applications has produced a large number of possible materials with potential to be applied in the temperature range of the motor proposed (RAM et al., 2018), there are still no commonly commercially available materials which could be applied. However, there are a few the promising magnetic materials that could be produced in a small laboratory scale and used in the TMM proposed, notably the intermetallic compounds MnAs, $\mathrm{La}(\mathrm{Fe}, \mathrm{Si})_{13}, \mathrm{Mn}_{3-\mathrm{x}} \mathrm{Fe}_{\mathrm{x}} \mathrm{Sn}$, and the $\mathrm{Gd}_{4.7} \mathrm{Nd}_{0.3} \mathrm{Si}_{4}$. From which the compound $\mathrm{Gd}_{4.7} \mathrm{Nd}_{0.3} \mathrm{Si}_{4}$ was selected, this magnetic material has a $T_{C}$ of $327.15 \mathrm{~K}$ that is well within the desired range; it presents a second-order thermomagnetic transition similarly to $\mathrm{Gd}$.

The $\mathrm{Gd}_{4.7} \mathrm{Nd}_{0.3} \mathrm{Si}_{4}$ used in the motor was initially proposed by Coelho (GAMA, personal communication, September 25, 2017) based around the classic $\mathrm{Gd}_{5} \mathrm{Si}_{4}$ intermetallic, which has a $T_{C}$ of $336 \mathrm{~K}$ (PECHARSKY; GSCHNEIDNER, 2007). This compound was modified by the addition of Neodymium, with $\left(\mathrm{Gd}_{5-\mathrm{X}} \mathrm{Nd}_{\mathrm{X}}\right) \mathrm{Si}_{4}$ and $\mathrm{x}=0.3$, which reduces the $T_{C}$.

The necessity of using a magnetic material that is not a common engineering material provided some additional and unexpected problems, one of which is the determination of its physical properties, since no detailed material characterization could be found in the literature. One of such properties was the magnetization of the material as a function of the applied magnetic field and at different temperatures, which was measured using a Physical Property Measurement System PPMS (Coelho, personal communication, February 5, 2013), and is presented in Figure 36. 
Figure 36: Magnetization of the intermetallic compound $\mathrm{Gd}_{4.7} \mathrm{Nd}_{0.3} \mathrm{Si}_{4}$ for a given applied magnetic field at different temperatures.

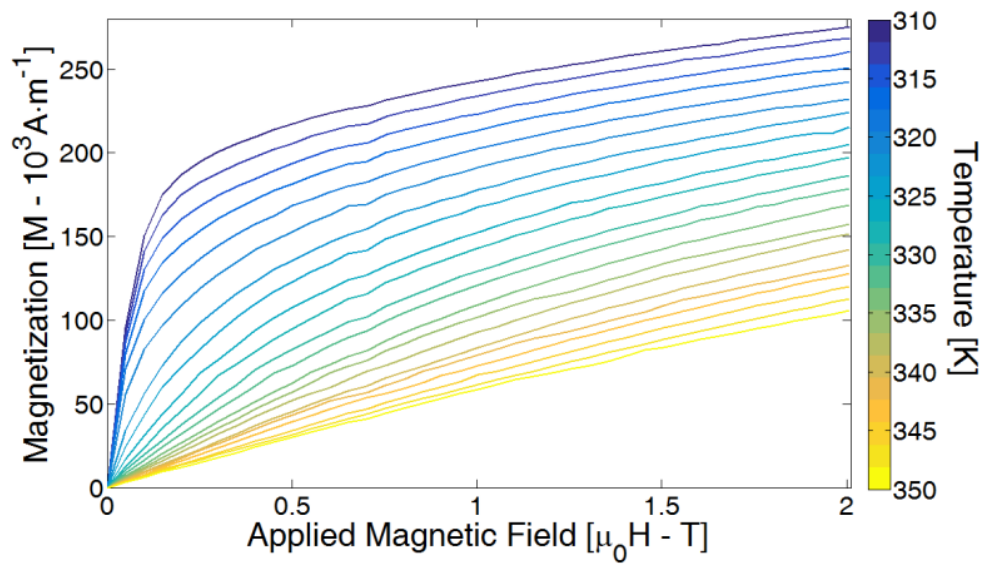

As this intermetallic compound presents a high hardness and a brittle structure it could not be easily machined into the magnetic plates to be used in the motor proposed, because of that the material was agglomerated with an epoxy resin to a percentage of $5 \%$ in weight. The details of the process used to produce the $\mathrm{Gd}_{4.7} \mathrm{Nd}_{0.3} \mathrm{Si}_{4}$ compound as well as the die pressure agglomeration process developed are presented in Appendix 1.

A laboratory scale proof of concept of the rotary type thermomagnetic motor was built, following the mechanical construction modeled, as presented in Figure 37. Based on the principles shown in chapter 3, the design can be divided into three basic systems: the magnetic field source, the heat transfer processes involved in the change of temperature of the magnetic material, and the system dynamics and power production. Each of these systems was modeled using computational tools. These models were then validated according to the data measured for the built proof of concept, as presented in the following chapters. 
Figure 37: The Rotary Thermomagnetic Motor built to demonstrate and validate the technology of TMM for the conversion of low-grade energy sources into useful mechanical energy.

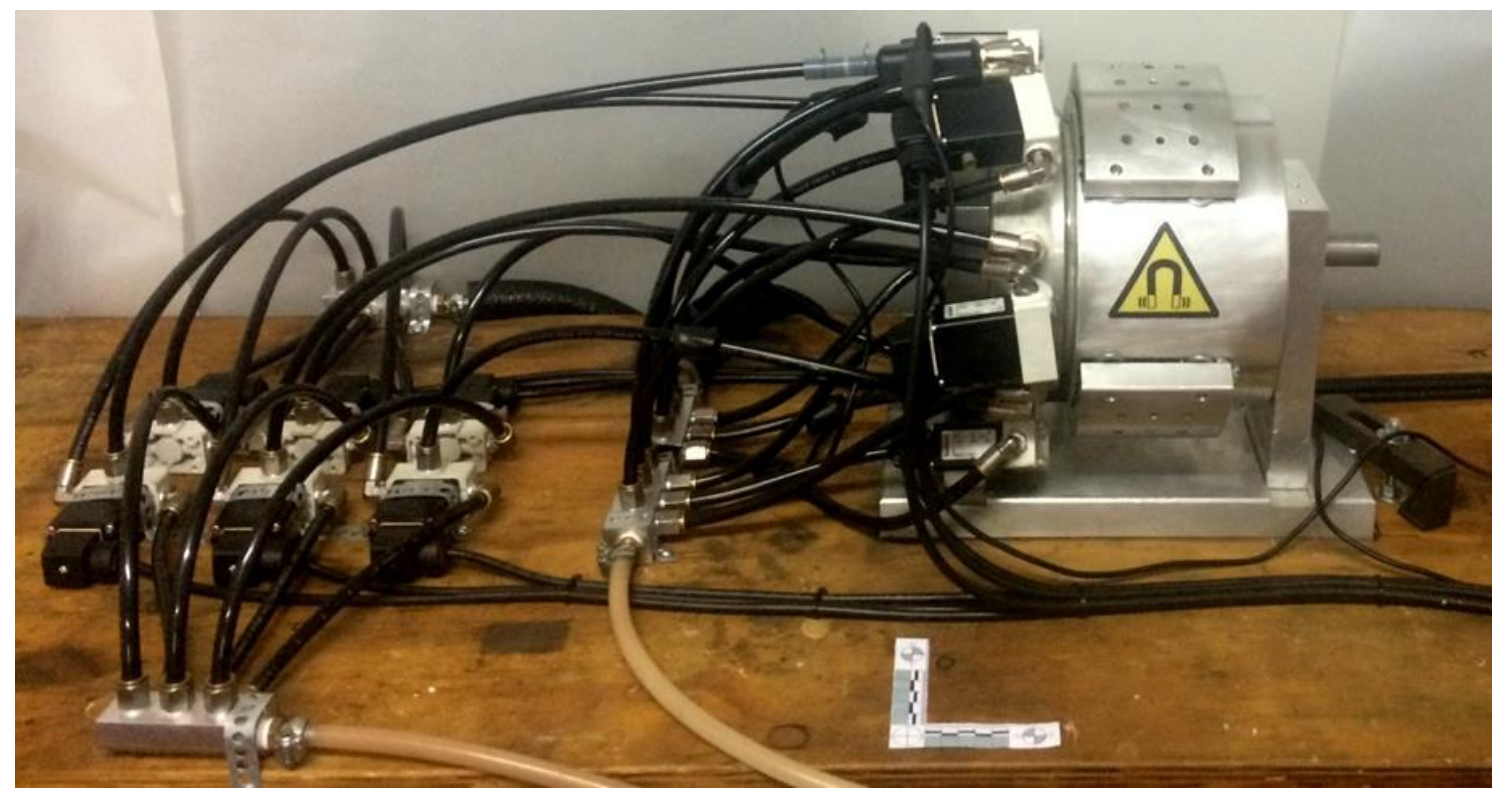

\subsection{The reporting of experimental data}

In general, the result of any experiment is only an estimate of the value of the measurand and therefore it is incomplete without a statement of the uncertainty of such estimate. This reporting of the experimental uncertainties is done throughout this thesis following the principles stated in the "Guide to the expression of uncertainty in measurement - JCGM 100:2008” (GUM) (JOINT COMMITTEE FOR GUIDES IN METROLOGY, 2008a).

The combined uncertainties of measurements are then characterized by the numerical values obtained by applying usual methods for the combination of variances. The GUM document proposes that this uncertainty propagation is based on replacing the experimental model by a first-order Taylor series approximation, referred to as the law of propagation of uncertainty, although higher orders of the series may be applied. In practice, only simple cases can be implemented through this method without making approximations, and the document is restricted to such cases.

As an alternative, numerical methods can be used to implement the propagation of distributions, specifically the Monte Carlo Method (MCM) is proposed by the GUM Supplement I JCGM 101.2008 (JOINT COMMITTEE FOR GUIDES IN METROLOGY, 
2008b). The MCM presents a more practical approach than the original GUM law of propagation of uncertainty, as it uses a probabilistic method, performing a characterization of the quantities measured, based upon the random sampling of the probability density function (PDF) of each uncertainty factor contributing to the measurement result. As this numerical simulation requires a high number of model evaluations (trials), a software application was developed in MATLAB®, based upon the work published by Fernández, Calderón and Díez (2009), which uses an adaptive approach to the MCM, adjusting the number of trials according to the stabilization criteria provided by a given numerical tolerance.

Finally, as described in the GUM, although the guide provides a framework for assessing the uncertainty, it cannot be a substitute for critical thinking, intellectual honesty, and professional skill. The evaluation of the uncertainty cannot become a routine task nor a purely mathematical one, as it depends on detailed knowledge of the nature of the measurand and of the measurement (JOINT COMMITTEE FOR GUIDES IN METROLOGY, 2008a). Or as put by Coleman and Steele (2009), experimentation is not just data taking, mostly the actual data-taking portion of a well-run experimental program generally constitute only a small percentage of the total time and effort expended. 


\section{THE MAGNETIC FIELD SOURCE}

Thermomagnetic motors of any kind work by the interaction between a magnetic material with varying magnetization, due to a change in its temperature, and an applied magnetic field gradient. This chapter describes the magnetic field sources used for the idealized motor test stand and the rotary Tesla type thermomagnetic motor. It also presents the main issues relating to the application of the magnetic field generator used, including, the design of the permanent magnets assemblies, the magnetostatic simulations performed using the software ANSYS Maxwell@, the assembly process, and the experimental validation of the simulations.

The magnetic field source for thermomagnetic motors can be provided by permanent magnets, electromagnets, and superconducting electromagnets. In this work, the only field generator studied was that provided by the arrangement of permanent magnets, because the other options operate with low efficiency or present high construction and operational costs, and complexity. According to Kitanovski et al. (2015), the thermodynamic efficiency of electromagnets must be considered as the ratio of the energy output (magnetization energy) and the energy input to the coil. The current applied to the coils in an electromagnet will induce Joule heating. Not only a valuable energy loss will occur, but also the magnetic field source may require additional cooling. In superconducting magnets, which can be treated as a particular type of electromagnets, the current may be kept circulating without electrical resistance losses. However, a cryogenic cooling system is required to generate the superconducting properties.

It is important to notice that, according to Kitanovski et al. (2015), the magnetic field source is the most expensive part of magnetocaloric energy conversion devices, especially those that are based on permanent or superconducting magnets. Hence the great care is taken in the search for an optimal assembly of permanent magnets, looking to obtain a costeffective and energy-efficient device.

The following designs were developed using Nd-Fe-B permanent magnets. These are currently the most powerful commercially available permanent magnets, having energy products $\left(B H_{m a x}\right)$ from 200 to $400 \mathrm{~kJ} \cdot \mathrm{m}^{-3}$, and also having a high remanence $(\mathrm{Br})$, a relatively 
high coercivity, reasonable temperature sensitivity, and good availability. The magnetic field gradients are produced by the use of assemblies of permanent magnets; these arrangements are designed to concentrate the magnetic field inside the region where the plates are located, following a principle similar to that proposed by Halbach (1980).

\subsection{Permanent Magnets Assembly for the idealized TMM}

The test stand projected to develop an idealized TMM, as presented in section 4.1, needs a magnetic field source capable of producing an elevated magnetic field gradient, allowing the system to measure the magnetic force produced by the interaction between this source and a small sample of magnetic material. It is also important that the field produced is concentrated in the region where the material sample is located, reducing the influence of any external field source or ferromagnetic material. A "horseshoe" type of magnets assembly is proposed to achieve these requirements. This assembly consists of two permanent magnets mounted to a plate of soft-ferromagnetic material. This design is presented in Figure 38, in which two 20x20x50 mm Nd-Fe-B magnets are mounted in opposing directions to a steel plate (darker color in the figure).

Figure 38: "Horseshoe" type of permanent magnets assembly designed for the test bench of an idealized TMM, dimensions shown in $\mathrm{mm}$.
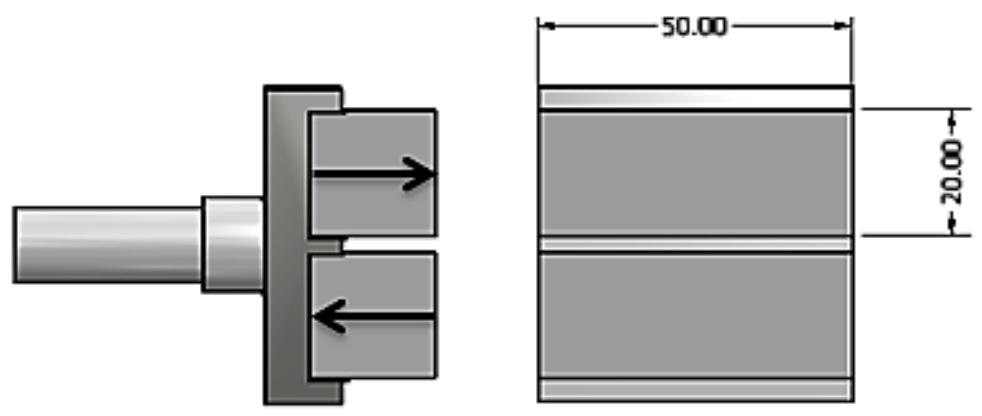

The assembly presented in Figure 38 will concentrate the magnetic field in the face of the magnets, as the soft-ferromagnetic material closes a magnetic circuit. The distance between the magnets will determine how dispersed is the field. If the magnets are in contact then a high field will be produced in a minimal region at the magnets contact edge. On the other hand, if the magnets are too far apart, then no field concentration will occur. In order to determine the adequate distance between the magnets, a $2 \mathrm{D}$ magnetostatic simulation was 
conducted using the software Ansys ${ }^{\circledR}$ Maxwell Electromagnetics Suite. This simulation has a more qualitative objective, as the validation of the exact field produced by the magnets assembly must be conducted using a 3D magnetostatic simulation. The design used for the 2D magnetostatic simulation is presented in Figure 39.a, in it is presented the orientation of the magnets, and the variable changed in the parametric simulation $\left(V_{a r}\right)$ that presents the distance between the two magnets. This parameter also changes the length of the supporting steel plate.

For this simulation, a mesh refinement of maximum length was applied to the design, with a maximum allowable mesh edge length of $0.5 \mathrm{~mm}$ to the solid elements and of $2 \mathrm{~mm}$ to the surrounding atmosphere. The convergence was controlled through the percentage energy error variation, using a convergence parameter of $0.005 \%$. The total number of elements in the mesh after convergence was around 48000 for all the simulations. From this first simulation, the applied magnetic field $(\mathrm{H})$ is chosen as the main output parameter, whose results are presented in Figure 39.b.

Figure 39: The design used in the 2D magnetostatic simulation (a) to determine the adequate distance between the magnets $\left(V_{a r}\right.$, in $\left.\mathrm{mm}\right)$, and the resulting applied magnetic field for this varying parameter (b).

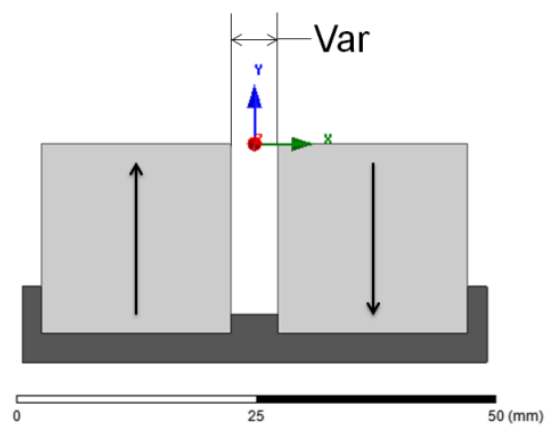

(a)

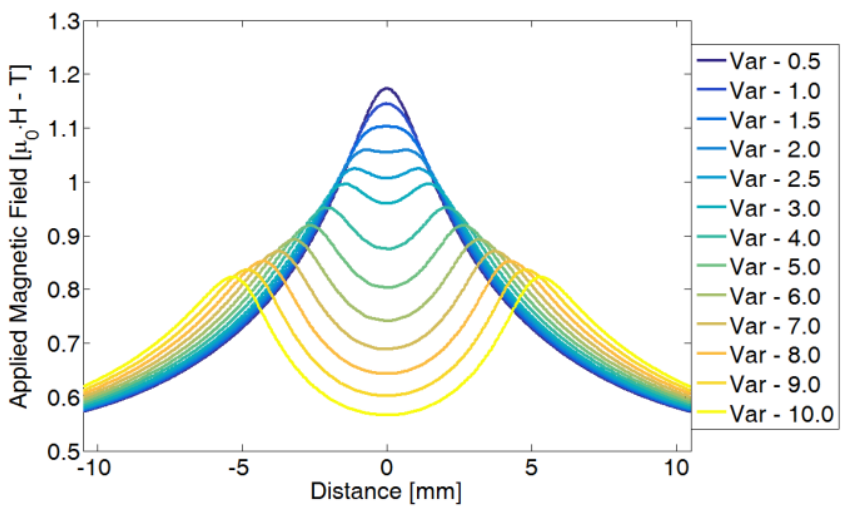

(b)

The resulting applied magnetic field from the simulation, presented in Figure 39.b is measured over a line in the x-direction (from the coordinates shown in Figure 39.a), at a distance from the magnets (in the y-direction) of $1 \mathrm{~mm}$. This result confirms the idea that a smaller distance between the magnets produces a more intense applied magnetic field, of up to $1.2 \mathrm{~T}$ in the simulation, although in a very concentrated region. On the other hand, if the distance between the magnets is too long then no significant concentration of field occurs, with the simulation results presenting two distinctive high peaks of $0.85 \mathrm{~T}$ for the distance 
$V_{a r}$ of $10 \mathrm{~mm}$. By analyzing the results, the value of $V_{a r}$ chosen is that of $2.5 \mathrm{~mm}$, as it provides a maximum field of up to $1 \mathrm{~T}$ in a more distributed region that is necessary for the tests of the idealized TMM.

The field distribution and the direction of the field lines are fundamental to the understanding of the expected magnetic force on the magnetic material tested in the built test stand. The induced magnetic field $(B)$ produced by the magnets assembly, with the chosen magnets distance $\left(V_{a r}\right)$ of $2.5 \mathrm{~mm}$, is presented in Figure 40.a. As seen in the figure there is a relatively uniform magnetic field around the center of the assembly, and this field decays steadily by the increase of the distance from the magnets. As the magnetic field closes through the air gap, the vector induced field lines presented in Figure 40.b will not be uniform or unidirectional. This is important to the determination of the internal magnetic field of the sample, as the demagnetizing field is dependent on the direction of the applied field and the geometry of the material.

Figure 40: Induced magnetic field $(B)$ by the "horseshoe" type of magnets assembly (a), and vector field lines (b), according to initial 2D magnetostatic simulation.

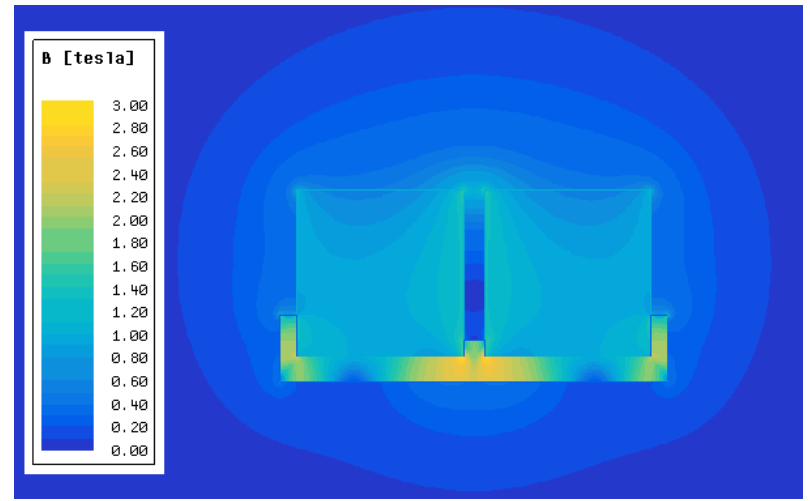

(a)

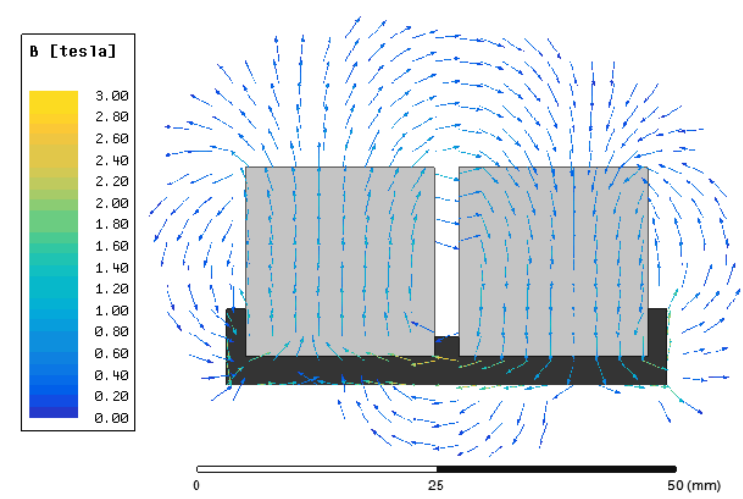

(b)

The analytical calculation of the magnetic force for the permanent magnets assembly proposed on the idealized TMM will be difficult, as seen by the vector field lines in Figure 40.b. Since both the direction and intensity of the applied magnetic field are changing with position, this calculation will demand the knowledge of the demagnetizing factor $(\gamma)$ that is changing with the position of the sample. The variable $\gamma$ could then be obtained experimentally, by the measurement of the magnetic force over a sample of known magnetization with the varying position, as done in the proposed test stand for the idealized TMM. 


\subsubsection{Experimental determination of the applied magnetic field.}

The validation of the results obtained from the magnetostatic simulations of the magnets assembly used in the idealized TMM requires the comparison of the magnitude of the applied magnetic field strength $\left(\mu_{0} H\right)$ obtained in the simulations to experimental results. The applied magnetic field produced by the magnets must then be measured as a function of the displacement of the magnet, taking as an initial position the face of the magnets and moving the assembly in the Y direction (as indicated in Figure 39.a).

The measurement of the applied magnetic field was done using a Hall effect sensor, capable of measuring the field with a good resolution and presenting a small uncertainty. The control system developed for the idealized Tesla type TMM, as presented in section 4.1, with the Hall effect sensor was positioned in the place of the sample holder. The Hall sensor voltage measurement and the control of the position of the magnets are done using a NI-USB-6363 data acquisition device, with an automatic experimental procedure developed through the LabVIEW software.

The Hall effect sensing element to be used in the experiments is the CY-SJ-362A, from ChenYang, which is chosen due to its small dimensions, good resolution, and linearity, and the capacity to measure applied magnetic field strengths of up to $3 \mathrm{~T}$. One key characteristic of this sensing element is that it provides a Hall voltage proportional to the applied magnetic field strength in a single orientation perpendicular to the sensing surface, due to its construction with a single-crystal semiconductor sensing component. However, this sensing element is not provided with a specific calibration curve, requiring calibration based on a known applied magnetic field strength to be used as a proper sensor.

Ideally, the calibration field would present a known strength with unidirectional field vector lines, to which the sensing element could be mounted perpendicularly. This procedure could then be done using a superconducting electromagnet, capable of producing the high applied magnetic field strengths obtained in the permanent magnets assemblies. As such equipment was not available the applied magnetic field strength produced by a base permanent magnets assembly as a function of its displacement was used as reference for the calibration of the Hall sensing element.

To obtain high strength and a highly unidirectional field, two high strength permanent magnets (NdFeB rated grad $50 \mathrm{M})$ are mounted with opposing orientations, as shown in 
Figure 41.a. The induced magnetic field $(B)$ produced by the magnets assembly can be compared to that produced by a single magnet, as shown in Figure 41.b. From which can be seen that the assembly produces a unidirectional field in the Y direction, with much stronger intensity than the maximum field obtained by a single magnet.

Figure 41: Vector induced field lines of two opposing NdFeB permanent magnets with dimensions of 20x20x100 mm (a), and of a single magnet with the same dimensions (b).

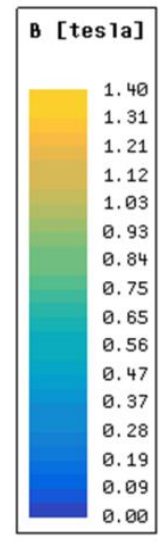

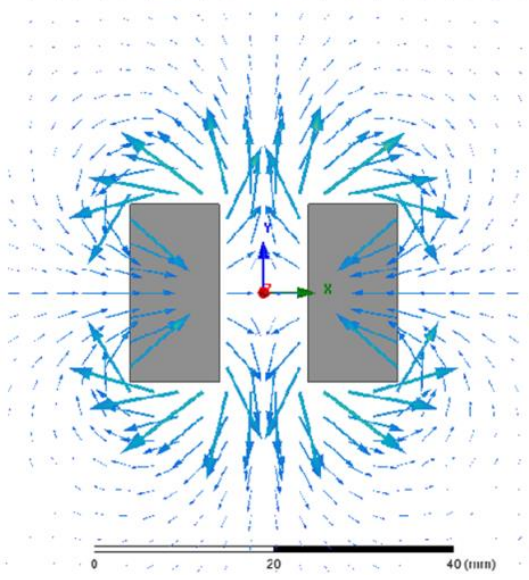

(a)

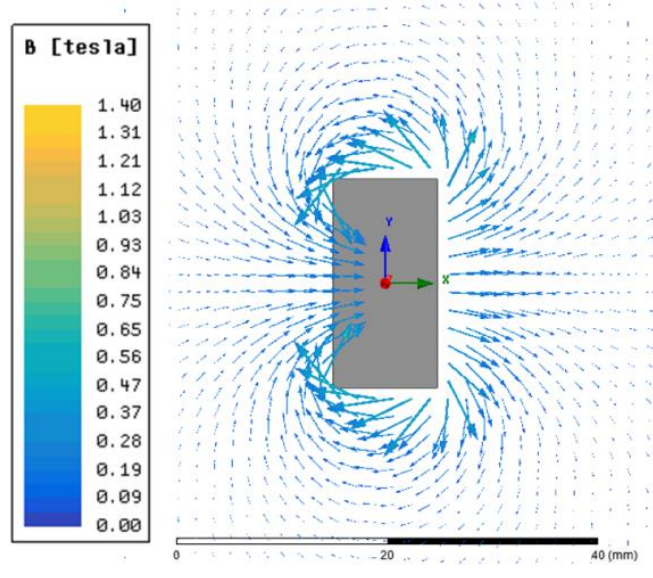

(b)

In Figure 41, the vector lines of the induced magnetic field (B) are plotted, obtained through a 2D magnetostatic simulation, with a mesh refinement of maximum length was applied to the design, with a maximum allowable mesh edge length of $1 \mathrm{~mm}$ to the solid elements and of $5 \mathrm{~mm}$ to the surrounding atmosphere. The convergence was controlled through the percentage energy error variation, using a convergence parameter of $0.1 \%$. The total number of elements in the mesh after convergence was around 15000 for the two opposing magnets assembly, and of 6500 for the single magnet. The results showed a good qualitative representation of reality.

The magnetic field strength produced by this base assembly is then determined using a portable Gaussmeter, model TLMP-Hall-15k-T0-E from GlobalMag. This instrument is capable of measuring fields of up to $1.5 \mathrm{~T}$, with a resolution $1.5 \mathrm{mT}$, a precision of $\pm 2 \%$ of the read value and $\pm 1 \%$ of the full scale. This digital instrument performs a uniaxial reading of the applied magnetic field, which is taken as a reference for calibration with the composed uncertainties of the measurement, as presented in Figure 42. 
Figure 42: Applied magnetic field strength as a function of the displacement of the reference permanent magnets assembly (Figure 41.a), used to determine the calibration curve for the Hall sensing element.

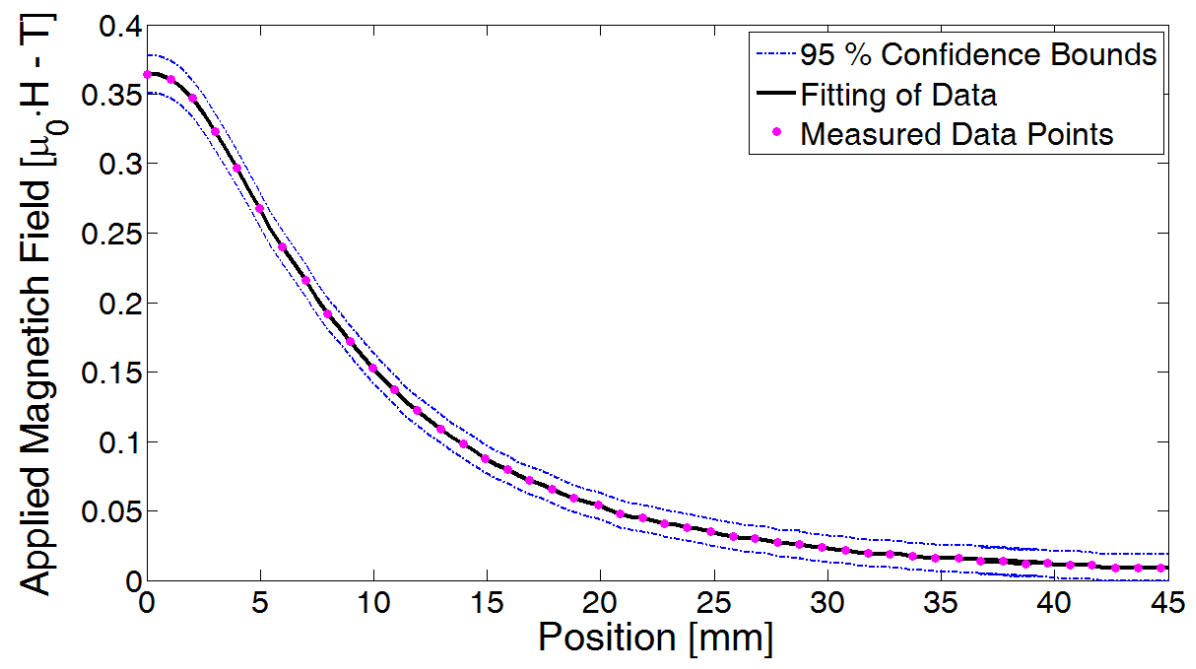

The applied magnetic field produced by the reference permanent magnets assembly was measured in the Y direction (Figure 41.a), with the sensor positioned in the center of the magnets assembly ( $X=0$ in Figure 41.a) and the measuring probe at the level of the face of the magnets ( $\mathrm{Y}=10 \mathrm{~mm}$ in Figure 41.a). From the initial position, the magnets are displaced $0.5 \mathrm{~mm}$ in each interaction and the measured applied magnetic field registered, the measured points shown in Figure 42 represent half the collected data points, as to avoid the excessive cluttering of the graph.

To determine the composed uncertainties of measurement the precision of the Gaussmeter used was combined with the uncertainty of positioning, using the fitting of the measured data. The total uncertainty of measurement was then determined using the Monte Carlo Method (MCM) of propagation of uncertainties, with the MATLAB®, based upon the work published by Fernández, Calderón, and Díez (2009). The probability density function (PDF) of the composed uncertainties then assumed a curvilinear trapezoidal shape, from which the $95 \%$ confidence bounds are determined, as presented by the dotted lines in Figure 42.

As shown, the Gaussmeter used was capable of producing a suitable calibration curve for the Hall sensor element, despite this, it could not be used directly in the measurement of the applied magnetic field produced by the permanent magnets assembly used in the idealized TMM. This is due to the hard and sizeable measuring tip of the device that could not be easily positioned for the measurement in three dimensions, necessary to calculate the 
magnitude of the applied magnetic field. Also, this device does not have a digital communication port, which is necessary to perform the automatic experimental procedure.

Using the measured applied magnetic field produced by the reference permanent magnets assembly the Hall sensing element can be calibrated. The calibration can be done by measuring the Hall sensing voltage produced by the element as it is mounted in the same position as the probing tip of the Gaussmeter, and again displacing the reference permanent magnets assembly. The resulting voltage curve is then plotted against the measured data points, as is presented in Figure 43, from which the linearity of the sensing element can be observed.

Figure 43: Calibration curve of the Hall effect sensing element CY-SJ-362A, from ChenYang, with the measured Hall sensor voltage and corresponding applied magnetic field strength.

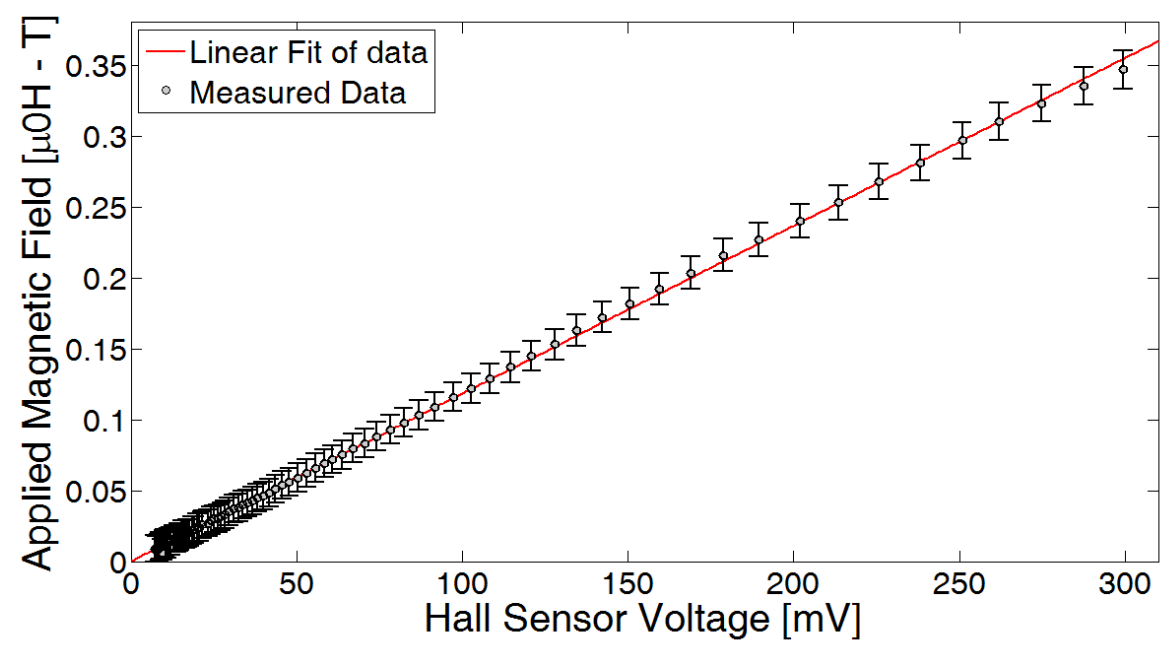

The corresponding calibration curve can then be fitted to a linear curve, as shown in Figure 43, from which the voltage to applied magnetic field strength equation is obtained, as presented below:

$$
\mu_{0} H=1.2 \cdot 10^{-3} \cdot V_{H a l l}+5 \cdot 10^{-4}
$$

One important detail to be observed from the calibration curve of the Hall sensing element is that the uncertainty in the calibration occurs mainly due to the reference applied magnetic field used. As the sensing voltage is measured using a NI-USB-6363 data acquisition device, which presents analog IO ports with Analog-to-digital converters of 16 Bits with adjustable gain, and excellent signal filtering capabilities, the uncertainty in the Hall sensing voltage measured would be of the order of $1 \mu \mathrm{V}$. 
As the uncertainty from the measured applied magnetic field is dependent on the intensity of the field measured, the standard uncertainty obtained using the calibrated Hall sensor will also present this characteristic. By plotting the calculated combined standard uncertainty against the applied magnetic field strength measured, as presented in Figure 44, the standard uncertainty is shown to grow exponentially. Using the fitting of the calculated standard uncertainty this value can be extrapolated for an applied magnetic field larger than the ones used in the calibration procedure.

Figure 44: The dependency of the standard uncertainty on the applied magnetic field for the sensor used to measure the magnetic field.

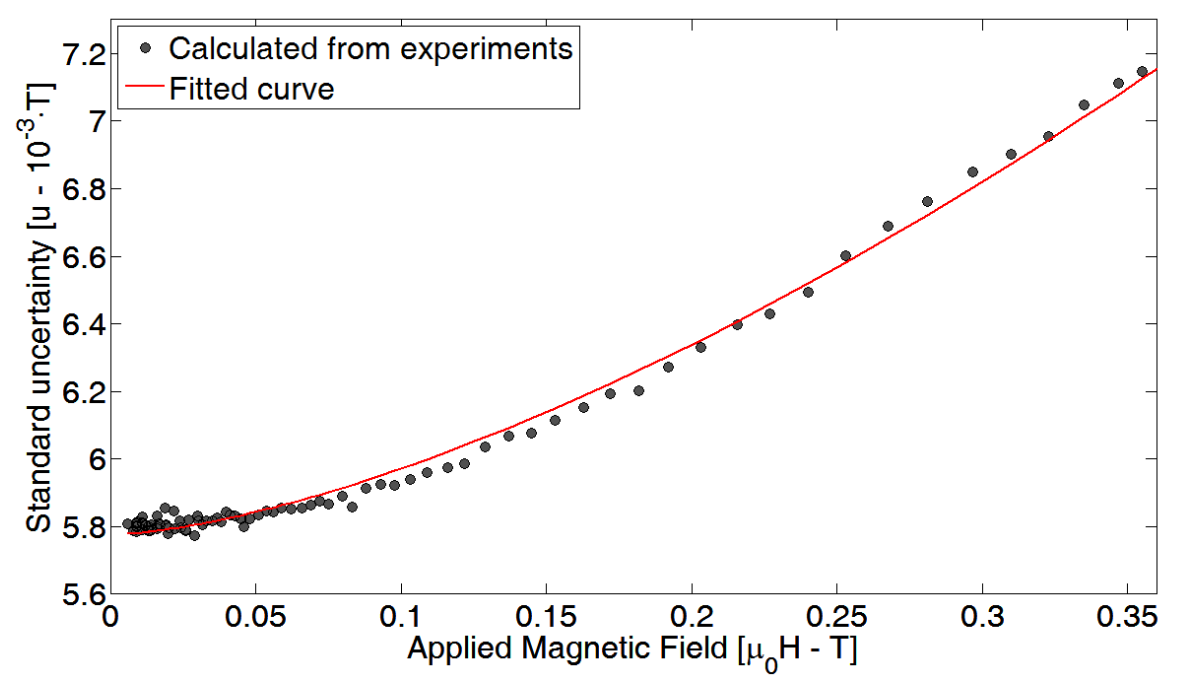

Using the calibrated Hall sensor the applied magnetic field strength produced by the "horseshoe" type of magnetic arrangement, used in the idealized TMM, can be measured. As noted before the Hall sensor used presents a uniaxial measurement, because of this characteristic the sensor must be positioned in three different orientations for the magnitude of the applied magnetic field strength to be computed. The sensor is initially positioned touching the magnets, in the position $\mathrm{Y}=0$ in Figure 39.a and the center of magnets assembly, Figure 45 presents the initial positioning of the hall sensor used. 
Figure 45: The experiment to measure the applied magnetic field produced by the "horseshoe" permanent magnets assembly used in the idealized TMM.

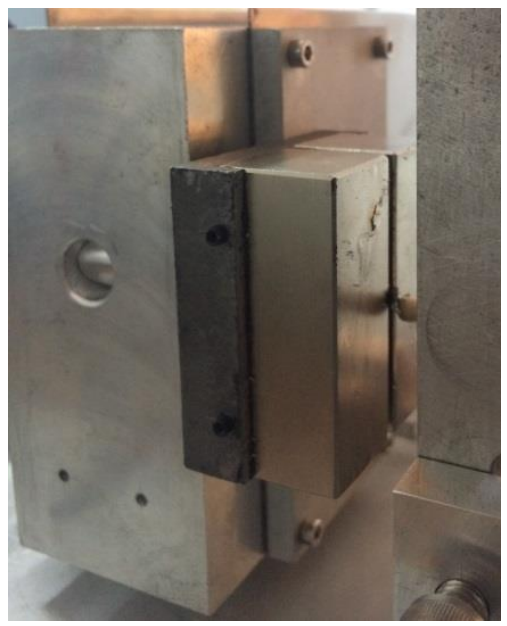

(a)

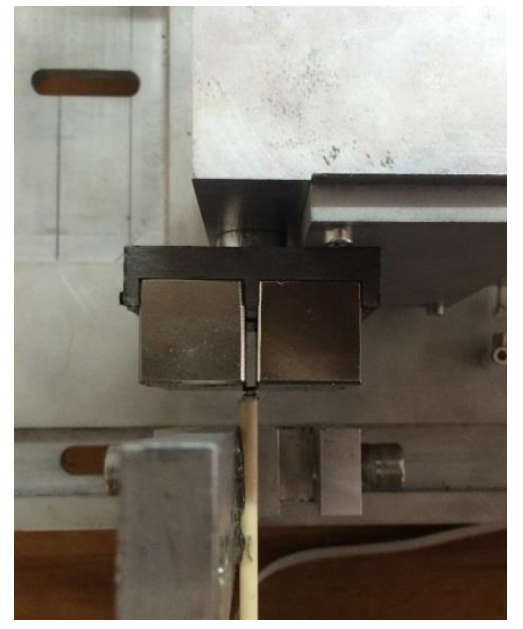

(b)

From the initial position, the magnets are displaced in $0.25 \mathrm{~mm}$ increments, while the data acquisition software registers both the position of the magnets and the measured applied magnetic field, converted from the measured Hall sensing voltage using the calibration curve. The experiment is repeated one time for each orientation, adjusting the Hall sensor each time. With that, the measured applied magnetic field obtained is presented in Figure 46. Only half of the collected data points are shown to reduce the cluttering of the graph. The error bars shown in the graph present the $95 \%$ confidence interval bounds, calculated from the uncertainty fit presented in Figure 44.

Figure 46: The measured applied magnetic field strengths as a function of the displacement of the "horseshoe" permanent magnets assembly, with the calculated uncertainties for each measured point.

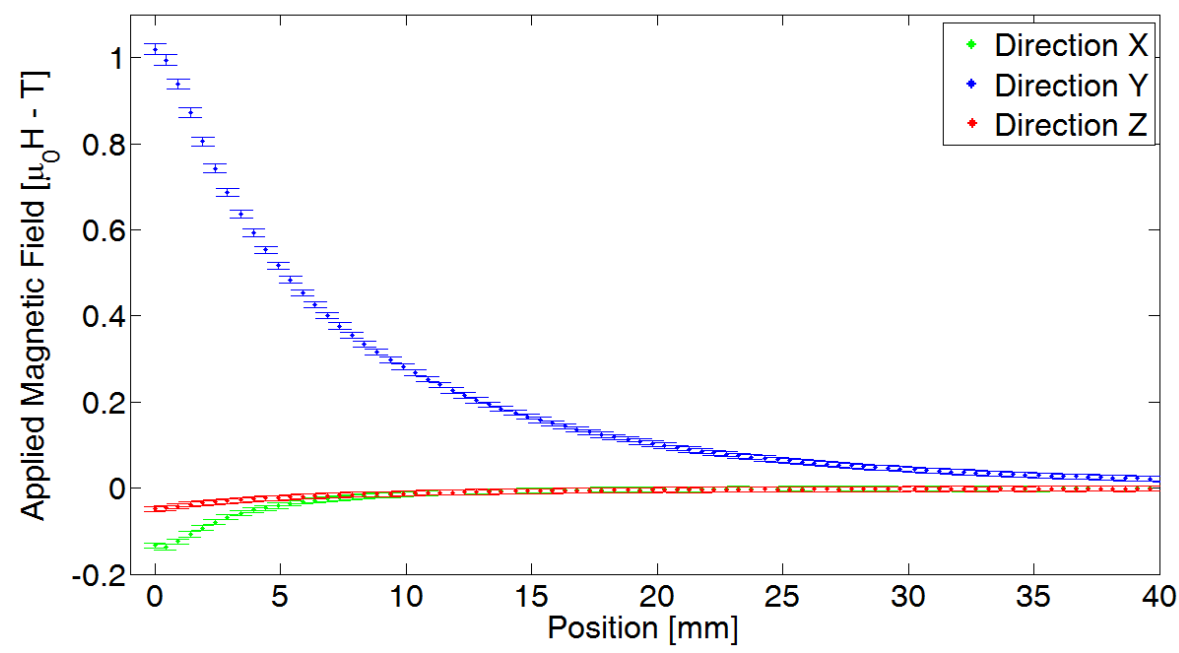




\subsubsection{Validation of the simulated applied magnetic field.}

The applied magnetic field determined experimentally, produced by the magnetic circuit used in the idealized TMM, can be used to validate the magnetostatic simulation method used. As the real application presents finite dimensions, the $2 \mathrm{D}$ approach used in the qualitative simulations of the magnets assembly, presented in Figure 39 and Figure 40, could not be expected to produce good quantitative results. Because of that a $3 \mathrm{D}$ magnetostatic simulation was conducted, using the modeling of the magnets assembly shown in Figure 47.

Figure 47: Model of the "horseshoe" type of magnets assembly used in the 3D magnetostatic simulation.

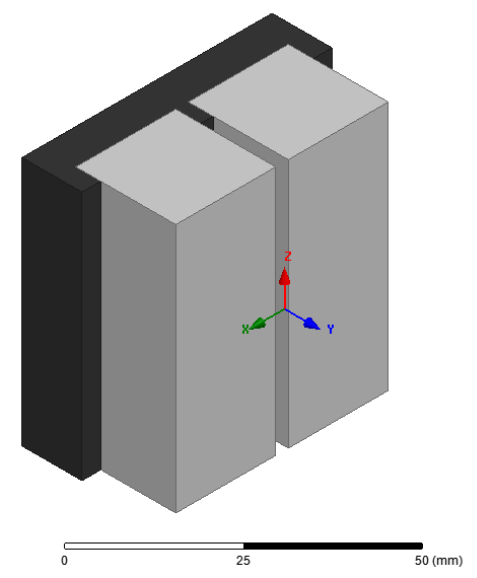

As this simulation has the aim to represent the behavior of the magnets assembly quantitatively, special care has been taken to use adequate materials and dimensions. The magnets modeled are the same that were used in the construction of the arrangement, being two $20 \times 20 \times 50 \mathrm{~mm}, \mathrm{NdFeB}$ with grade $50 \mathrm{M}$, that according to the ChenYang catalog present a nominal remanence $\left(B_{r}\right)$ of $1.43 \mathrm{~T}$, coercivity $(H c)$ of $1069 \mathrm{kA} \cdot \mathrm{m}^{-1}$, maximum energy product $\left(\mathrm{BH}_{\mathrm{MAX}}\right)$ of $398 \mathrm{~kJ} \cdot \mathrm{m}^{3}$, and maximum working temperature of $100^{\circ} \mathrm{C}$. The soft steel material used in the simulation is the SAE 1010, with the non-linear BH curve presented in the Ansys Maxwell software.

The simulation is done using a mesh refinement of maximum length, with a maximum allowable mesh edge length of $0.5 \mathrm{~mm}$ to the solid elements and of $5 \mathrm{~mm}$ to the surrounding atmosphere. The convergence was controlled through the percentage energy error variation, using a convergence parameter of $0.01 \%$. The total number of elements in the mesh after convergence was of 2264717. 
The magnitude of the applied magnetic field obtained through the 3D magnetostatic simulation can then be compared to that calculated from the field measurements. The experimental magnitude of the applied magnetic field is calculated from the vector addition of the measured applied magnetic field in the three orientations; this calculation also includes the combination of the uncertainties in each measurement, done using the Monte Carlo method of propagation of uncertainties. The results can then be compared in the graph shown in Figure 48, in it the error bars shown in the graph present the 95\% confidence interval bounds.

Figure 48: The magnitude of the applied magnetic field produced by the "horseshoe" type of permanent magnets assembly used in the idealized TMM.

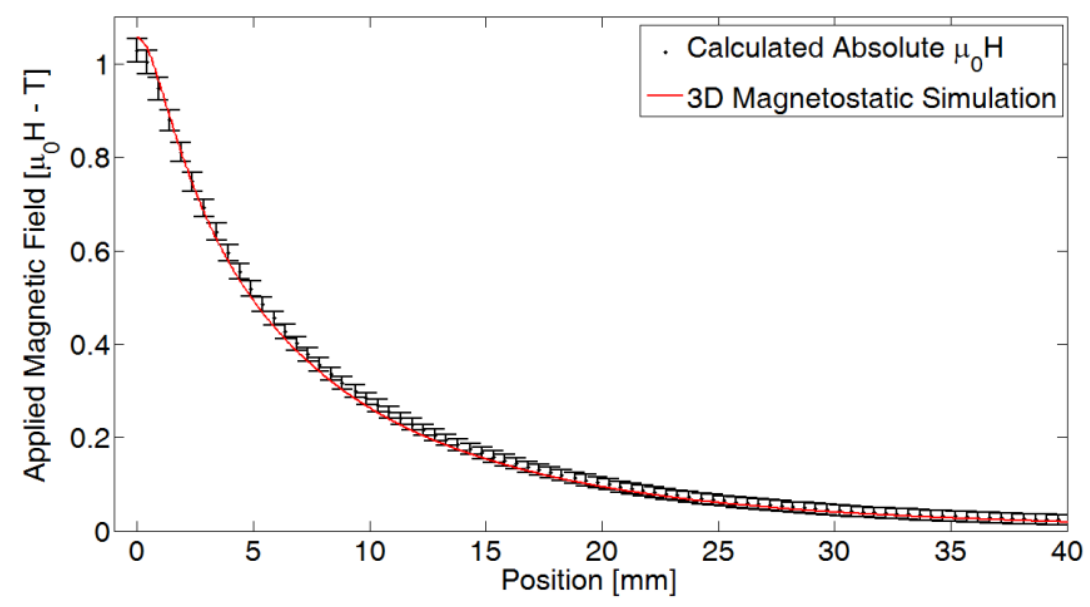

From the results presented in Figure 48, it can be seen that there is a good agreement between the magnitude of the applied magnetic fields obtained through the magnetostatic simulation and the measured results, as the simulation results are within the uncertainty bounds of the magnitude calculated from the experimental data for every data point. Although the magnitude of the applied magnetic field is only one of the many parameters calculated in the simulation, it is one of the fundamental quantities necessary to determine the magnetization work of a magnetic material, as shown in equation 3.1. Thus, it can be concluded that for the design shown the 3D magnetostatic simulation made using the Finite Element Magnetic analysis (FEM) software Ansoft Maxwell is experimentally validated. 


\subsection{Permanent Magnets Assembly for the Rotary Thermomagnetic Motor}

The magnetic field source for the rotary thermomagnetic motor is also a permanent magnets assembly, as previously noted this assembly must produce the highest possible magnetic field gradients to produce high forces in the motor, while also reducing as much as possible the total volume of magnets used. The magnets arrangements are then designed to concentrate the magnetic field inside the region where the plates are located, following a principle similar to that proposed by Halbach (1980). According to this principle, the magnets are oriented to direct the field lines in the desired direction. The design proposed is focused on producing the magnetic field inside the annular region where the magnetic material plates are located on the rotary TMM.

The magnets used in this assembly will be the same used in the permanent magnets assembly for the idealized thermomagnetic motor, $\mathrm{NdFeB}$ permanent magnets with grade $50 \mathrm{M}$ and dimensions of 20x20x50 $\mathrm{mm}$. The magnets orientations are then shown in Figure 49.a, with 13 orientations and forming an annular region with the angular arc of $92.4^{\circ}$ where the magnetic field is concentrated. The proposed design for the rotary thermomagnetic motor uses magnetic plates with a length of $0.1 \mathrm{~m}$, so for the permanent magnets assembly to cover the plates entirely, two permanent magnets (with the length of $50 \mathrm{~mm}$ ) are used in each orientation, to a total of 26 magnets in the assembly. To study the magnetic field produced by the assembly the 3D magnetostatic simulation with the software Ansoft Maxwell method is used since it was previously validated for a similar problem, as presented in the previous section. The model of the magnets assembly used in the simulation is shown in Figure 49.b. 
Figure 49: The permanent magnets assembly used in the rotary TMM, with the orientation of the magnets forming an annular region where the magnetic field is concentrated (a), and the model of the assembly used in the $3 \mathrm{D}$ magnetostatic simulation (b).

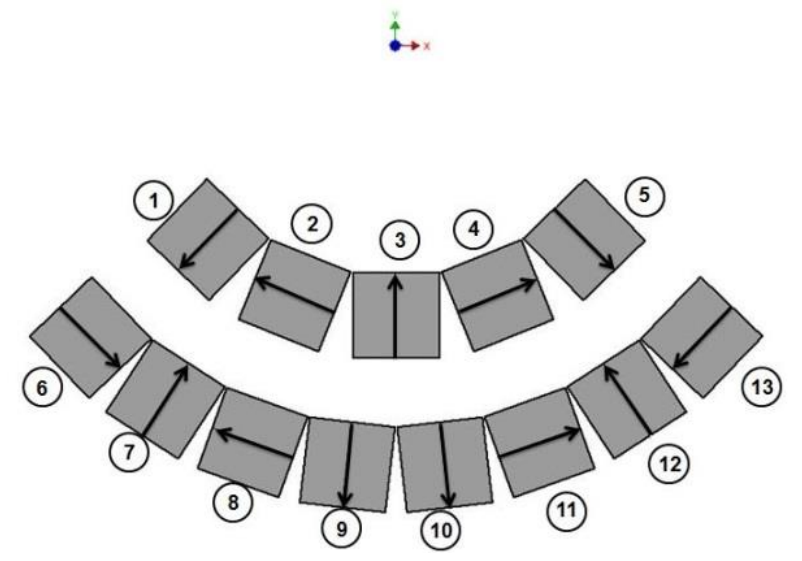

(a)

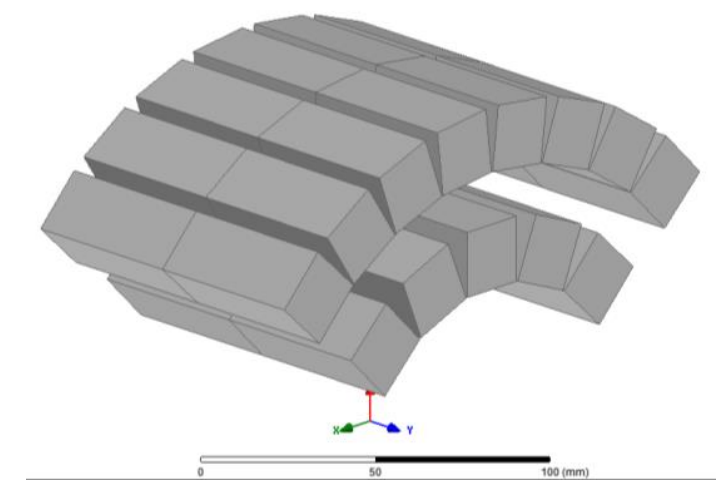

(b)

The magnetostatic simulation is done using a mesh refinement of maximum length, with a maximum allowable mesh edge length of $1 \mathrm{~mm}$ to the solid elements and of $10 \mathrm{~mm}$ to the surrounding atmosphere. The convergence was controlled by the percentage change of the energy error, using a convergence parameter of $0.05 \%$. As this simulation contained a larger number of parts, the convergence parameter had to be relaxed from the $0.01 \%$ used for the simulation of the "horseshoe" type of magnets assembly of the idealized TMM. The total number of elements in the mesh after convergence was of 1188569.

As a result from the $3 \mathrm{D}$ magnetostatic simulation, the induced magnetic field (B) produced by the assembly is presented in Figure 50.a, which shows the concentration of the field in the desired annular region, producing field intensities of up to $1.2 \mathrm{~T}$ in the region closer to the magnets. The field concentrating assembly proposed then shows a promising potential to be used in the rotary thermomagnetic motor, though with a noticeable caveat, by orientating the magnets in such positions large forces are induced in each magnet, as shown in Figure 50.b where the vectors indicate the direction and intensity of each force. Because of this, the construction of this permanent magnets assembly presents a challenging problem. 
Figure 50: The results from the 3D magnetostatic simulation, in (a) the induced magnetic field (B) produced by the assembly, as the view from a section plane of the model, in (b) the magnetic force induced in each permanent magnet from positioning in the assembly, values shown in Newtons.
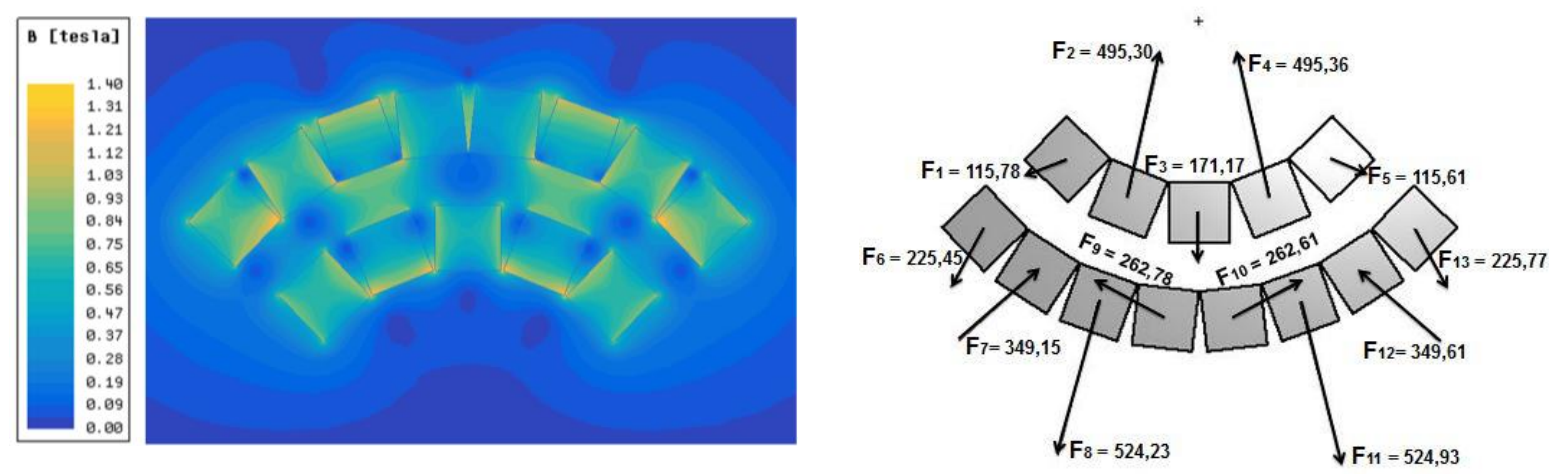

(a)

(b)

To position the magnets in the assembly aluminum support pieces are fabricated, these supports are intended to hold the magnets in the orientation necessary for the assembly, while also providing the connection from the magnets assembly to the rotor axis of the motor. Initially, an attempt was made to glue the magnets into the supports, using the high strength Loctite 330 acrylic adhesive, while the adhesive proved to be strong enough to hold the expected load this attempt was not successful, due to the separation of the protective coating, used to avoid corrosion of the $\mathrm{NdFeB}$ permanent magnets. As an alternative the magnets were drilled, using an electrical discharging machine (EDM) and the spark eroding process, producing a hole with a $4 \mathrm{~mm}$ diameter in the magnets, which were then used to bolt the magnets into the aluminum support. The mounting process was conducted using a milling machine, with specially designed clamps. After the assembly the magnets were covered with a protective paint layer, to avoid the corrosion of the permanent magnets, the built magnets assembly is then presented in Figure 51. 
Figure 51: Picture of the built magnets assembly, with a protective paint to avoid corrosion of the NdFeB permanent magnets.

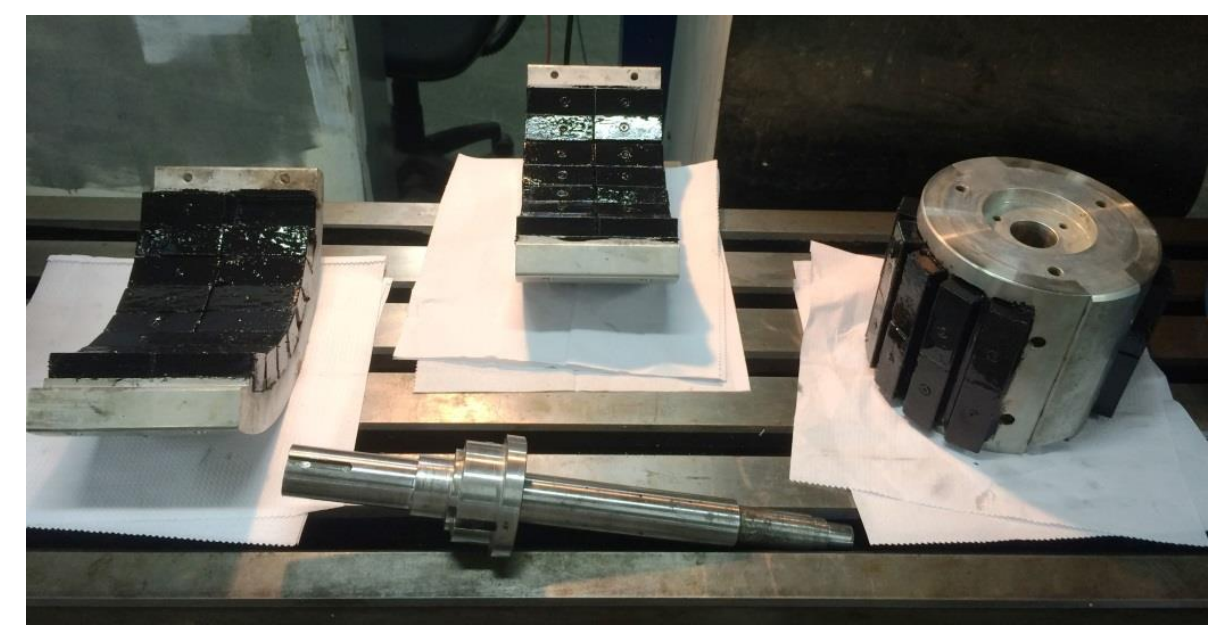

As the construction process of the permanent magnets assembly demanded the drilling of the magnets, and due to observed variations in the precision of the construction process, the assembled magnetic circuit could not be expected to produce the same results as those produced by the 3D magnetostatic simulation. To solve this inconsistency an experiment was conducted to measure magnetic field produced by the magnets assembly, and by using the results of this experiment, the simulation could be modified to correspond to the magnets assembly built.

To measure the magnitude of the applied magnetic field, produced by the built magnetic circuit, as a function of the angular position of the assembly, the magnetic circuit was fixed to an indexing head of a milling machine, used to control the angular position. Then to determine the actual angular position of the magnets a rotary encoder was mounted to the rotor axis, the encoder used was the E50S8-1000-3-T-24, from Autonics, which has a base resolution of 1000 pulses per revolution $\left(0.36^{\circ}\right)$, by using the quadruple precision reading $(4 \mathrm{X})$ the resolution can be improved to 4000 points per revolution $\left(0.09^{\circ}\right)$. The applied magnetic field strength was then measured using the calibrated Hall sensor CY-SJ-362A, from ChenYang, with the sensor fixed to the spindle axis of the milling machine, which allowed the positioning of the sensor using the mill controls.

As the Hall sensor used presents a uniaxial reading, the measurement was conducted three times, changing the orientation of the sensor to measure the applied magnetic field in the three directions. Then using the measured applied magnetic field strength for the three 
Cartesian directions the magnitude of the field was calculated, with the compounding uncertainty of measurement obtained from the sensor calibration, as shown in the previous section.

The magnitude of the field was then obtained for a series of varying positions of the Hall sensor, changing the gap between the sensor and the magnets, for gaps of 3, 5.5, 8, 10.5, and $13 \mathrm{~mm}$, and also the depth of the measurement, measured from the face of the magnets assembly into the area of field concentration, for the depths 0 (right at the corner of the magnets), 12.5, 25, 37.5, 50, and $62.5 \mathrm{~mm}$. Resulting in a total of 30 individual positions, for which the sensor had to be oriented in three directions, to a resultant 90 individual measurements. Then using this data, the applied magnetic field could be mapped, using a surface interpolation technique, as shown in Figure 52.

Figure 52: The applied magnetic field $\left(\mu_{0} H-\mathrm{mT}\right)$ produced by the magnets assembly, with the field mapping based on the experimental measurement, for a distance of $12.5 \mathrm{~mm}$ from magnets faces.

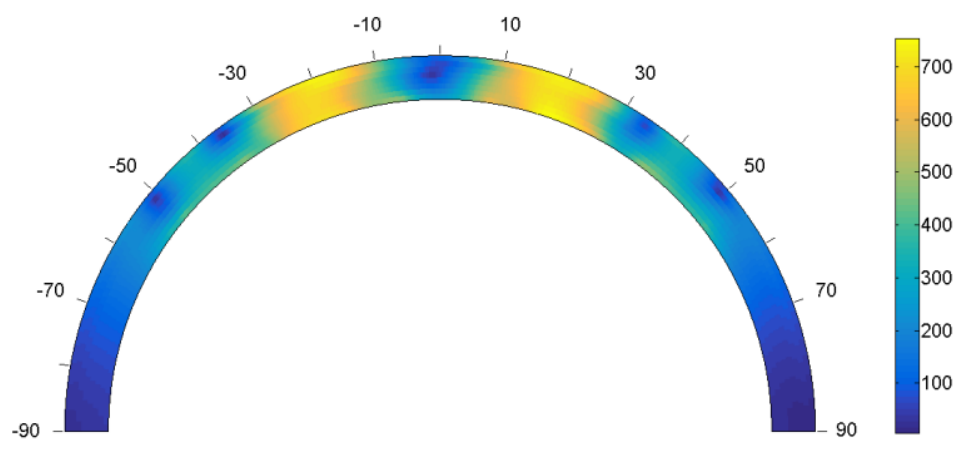

The mapping of the magnetic field obtained experimentally can then be compared to the simulation result shown in Figure 50.a. This comparison indicates that, despite the changes made in the magnets assembly, due to the fabrication process, the overall shape of the magnetic field was similar, but with reasonable reduction of the maximum applied magnetic field strength. With the measured data, the simulation can then be adapted to represent better the field developed by the built magnets assembly. A reference position of measurement must be assumed, which is used to compare to the simulation results. The chosen reference was that with the sensor at the middle position between the internal and the external magnets assembly, a gap of $8 \mathrm{~mm}$ from the lower magnet to the sensor face, and for the measurement depth, the distance of $12.5 \mathrm{~mm}$ from the face of the magnets was chosen.

The changes made in the simulation model, presented in Figure 49.b, were to remove the material that had to be drilled from the magnets for the mounting process and then to 
explore the possibilities of small misalignments between the external and the internal magnets arrangement. These simulations were conducted using the same mesh refinement and convergence parameters as those used for the initial simulations. From the results, it was verified that the actual distance between the internal and the external magnets arrangements was $1.5 \mathrm{~mm}$ greater than the initial simulation. Also, the results show that other small misalignments or possible small deformations would have a small impact on the magnitude of the applied magnetic field, resulting in changes that would be within the uncertainty of the measured data. The comparison between the results calculated from the measured data, the initial simulation, and the corrected simulation are shown in Figure 53.

Figure 53: The magnitude of the applied magnetic field produced by the permanent magnets assembly used in the Rotary TMM, for a line at the center of the annular gap region, as indicated in the inset in the figure, and at the center of the assembly.

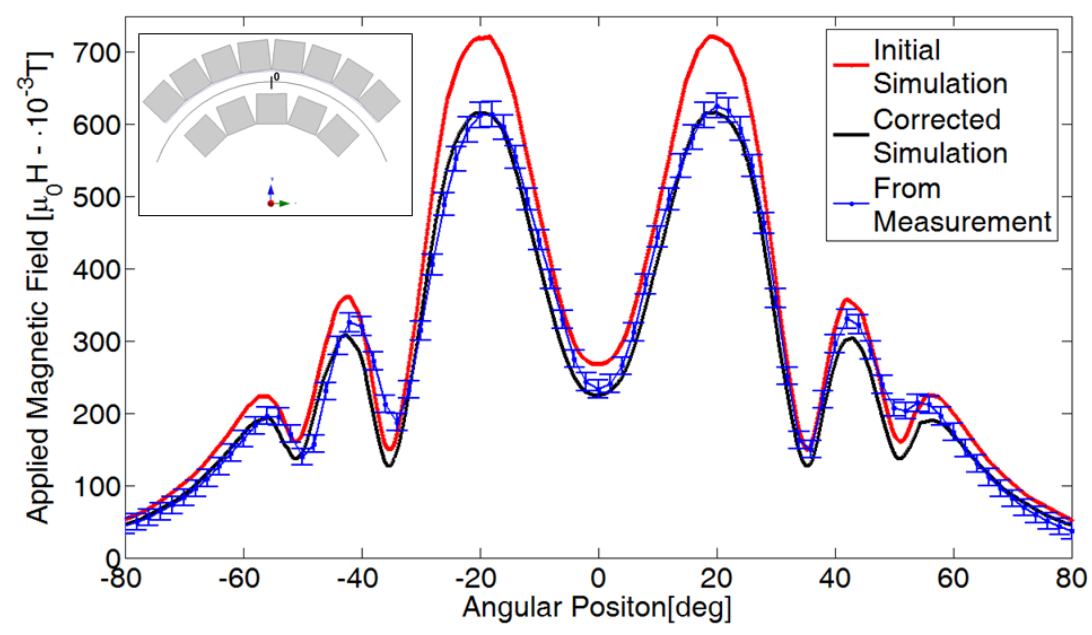

From these results, it can be concluded that an experimentally validated 3D magnetostatic simulation was obtained for the permanent magnets assembly used in the rotary thermomagnetic motor. Though some changes had to be made in the initial simulation, for it to correspond to the measured data, these changes were essentially geometrical and made the model approach the built permanent magnets assembly. 


\section{HEAT TRANSFER IN THE THERMOMAGNETIC MOTORS}

Thermomagnetic motors depend on the variation of the temperature of a magnetic material to produce work, this change in temperature is in essence the phenomenon that determines the amount of heat received and/or released by that material and therefore the amount of energy that is available to be converted into mechanical work, which could be calculated through the equation defining the specific heat:

$$
q=c \cdot \int_{T_{L}}^{T_{H}} T
$$

However, the specific heat of the magnetic material will not be constant for the cycle of the thermomagnetic motor, as it depends on the temperature of the material, and on the applied magnetic field, as shown in Figure 54 for Gadolinium. There is a peak on the specific heat capacity curve as the material changes its magnetization state, for the $\mathrm{Gd}$ at the $T_{C}$ of $293 \mathrm{~K}$. As the applied magnetic field increases the peak is lowered and broadened, while the peak temperature presents a small shift towards higher temperatures, due to the stabilization of the ferromagnetic phase of the material caused by the applied magnetic field. At large fields, the thermomagnetic phase change is suppressed. This behavior is very typical of second-order transition materials (SMITH et al., 2012).

Figure 54: Specific heat capacity as a function of the temperature for Gadolinium, for applied magnetic field strengths from 0 to $1.4 \mathrm{~T}$.

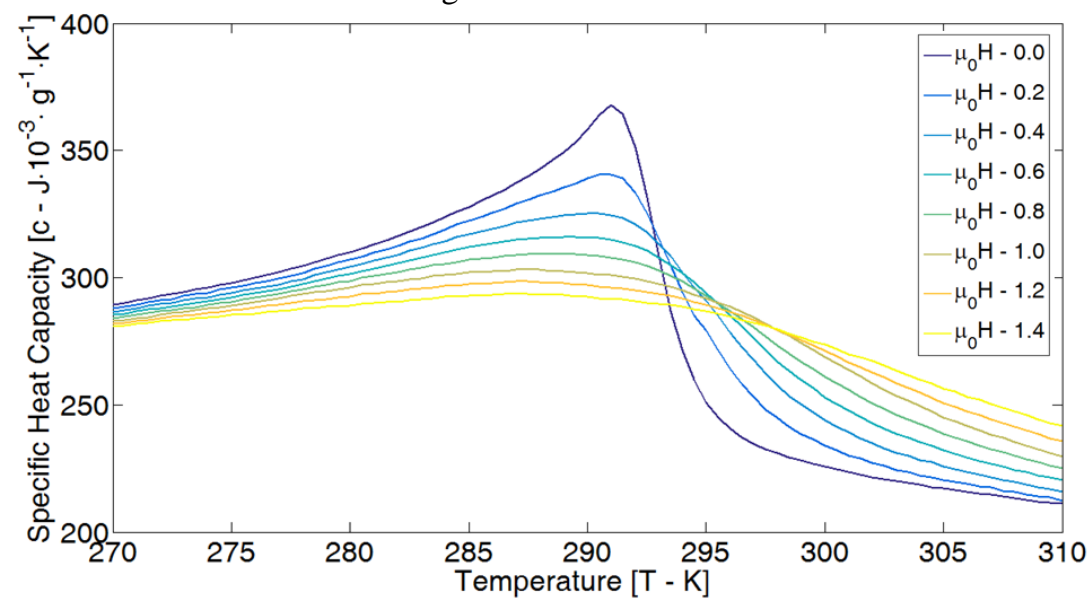

Source: Own authorship with data from Risser et al. (2012, 2013). 
For the $\mathrm{Gd}_{4.7} \mathrm{Nd}_{0.3} \mathrm{Si}_{4}$ compound used in the developed thermomagnetic motors, the specific heat capacity dependency on the applied magnetic field and the temperature was not available in the literature. This curve was then measured by Gandra (personal communication, September 2015), using a previously developed apparatus capable of measuring the specific heat change with temperature using a heat flux sensor, which is then mounted inside a superconductive electromagnet to provide the field swapping (MONTEIRO et al., 2014). The data obtained in the measurement is presented in Figure 55, from which the critical temperature $T_{C}$ is determined to be in the range of 320 to $325 \mathrm{~K}$, close to the expected $327 \mathrm{~K}$. The data also show the change in the heat capacity caused by the applied magnetic field, displaying a second-order transition behavior, similar to that of the Gd.

Figure 55: Specific heat capacity as a function of the temperature for the compound $\mathrm{Gd}_{4.7} \mathrm{Nd}_{0.3} \mathrm{Si}_{4}$, for two different applied magnetic fields.

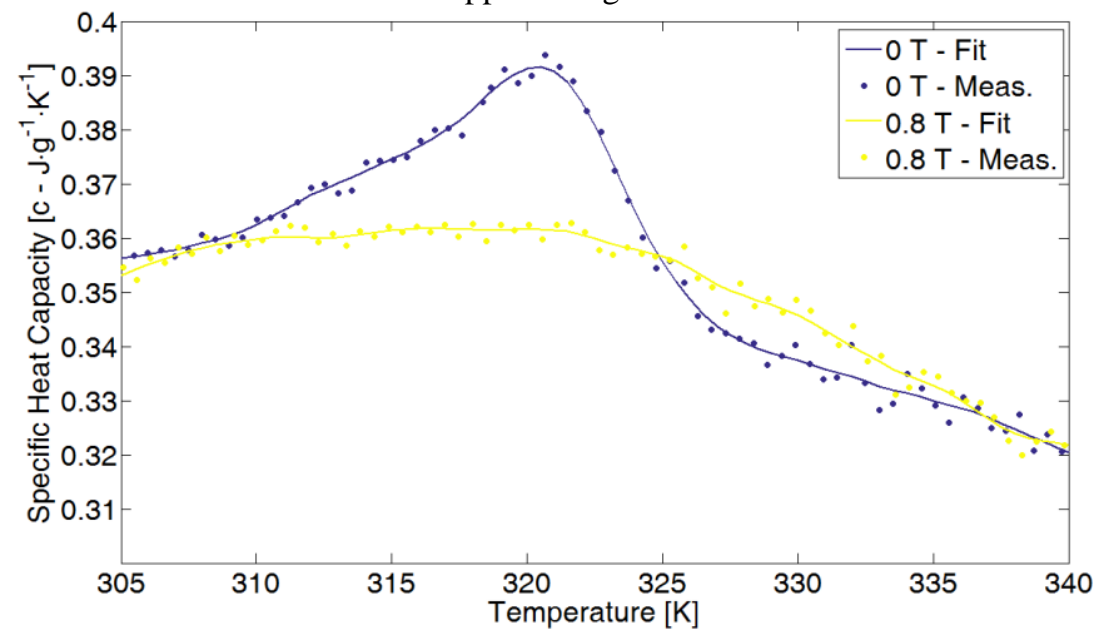

Source: Own Authorship, with data from Gandra (personal communication, September 2015).

With the known change in the specific heat, and by assuming the material in thermal equilibrium with the heat sink $\left(T_{L}\right)$ in the start of the cycle, and again in equilibrium with the heat source $\left(T_{H}\right)$ at the end, the amount of energy available to conversion could be calculated through equation 6.1. However, the assumption of thermal equilibrium in the start and end of the cycle is also associated to the assumption of a quasi-static and purely reversible transformation, with heat being conducted with no temperature difference between the material and the heat sources, which can only be assumed for infinitely large heat transfer times. 
For the idealized Tesla type of thermomagnetic motor previously shown the source of heat is not taken into account, this way the temperatures used to the define the Carnot efficiency are effectively the extreme temperatures of the material. This approach allows the determination of the specific work produced, to calculate the internal thermal efficiency of the motor, and to calculate the efficiency relative to Carnot, but not the determination of the power output. To adequately estimate the power associated with the thermodynamic cycle, that approach must be expanded by introducing a thermal exchange model as well as the finite change rate of the relevant quantities (ALMANZA et al., 2017). With that estimating the degree of departure from a thermal equilibrium condition between the magnetic material and the heat sources, and therefore taking into account the difference in temperature between the material and the reservoirs, given by the applied magnetic field change rate and the thermal exchange process efficiency.

Adequate analytical models of the heat transfer in thermomagnetic motors are complex to develop, beyond the complexities encompassed in modeling a conventional heat transfer system, the heat transfer in these devices is fundamentally happening in a transient state, during the cycling of the temperature. Furthermore, the magnetic materials are undergoing a phase change in each heating cycle, from a ferromagnetic to a paramagnetic state due to the thermomagnetic effect, which is happening in the solid state. This phase change also alters the specific heat of the material, as shown in Figure 54 and Figure 55, modifies the thermal conductivity of the material, and commonly also changes the volume and density of the material (GAMA et al., 2009), all of which are dependent on both the varying temperature of the material and on the applied magnetic field. Despite these challenges, through the use of numerical models, an adequate approximation of the heat transfer in the thermomagnetic devices can be obtained, specifically through finite element methods.

\subsection{Heat Transfer in the Rotary Thermomagnetic Motor}

The heat transfer in the developed rotary thermomagnetic motor occurs through the flow of a heat transfer fluid, which is pumped and circulates through internal channels in the plate of magnetic material, therefore developing a forced convective heat transfer process. The convection heat transfer depends on the characteristic fluid properties of dynamic 
viscosity $(\mu)$, thermal conductivity $(k)$, density $(\rho)$, and specific heap $(c)$, as well as the fluid flow properties of fluid velocity $(V)$ and developed turbulence levels $(\delta V)$. It also depends on the geometry and roughness of the solid surfaces (ÇENGEL, 2007). Despite its complexity, the rate of convection heat transfer $\left(\dot{Q}_{\text {conv }}\right)$ is observed to be essentially proportional to the temperature differences, and it is expressed by Newton's law of cooling as:

$$
\dot{Q}_{\text {conv }}=h \cdot A_{s} \cdot\left(T_{s}-T_{\infty}\right)
$$

Where $h$ is the convection heat transfer coefficient $\left(\mathrm{W} \cdot \mathrm{m}^{-2} \cdot \mathrm{K}^{-1}\right), A_{S}$ is the heat transfer surface area $\left(\mathrm{m}^{2}\right), T_{S}$ is the temperature of the surface $(\mathrm{K})$, and $T_{\infty}$ is the temperature of the fluid sufficiently far from the surface $(\mathrm{K})$. Importantly the factor $h$ will depend on all the flow conditions previously noted, being determined experimentally. For the flow of liquids, common values of $h$ vary between 50 and $20000 \mathrm{~W} \cdot \mathrm{m}^{-2} \cdot \mathrm{K}^{-1}$ (ÇENGEL, 2007).

In the analysis of the heat transfer in the rotary thermomagnetic motor the complexity of modeling the convective heat transfer is added to the challenge of modeling the magnetic materials undergoing a thermomagnetic cycle. Because of that, a complementary approach between computational simulations and experimental analysis was adopted, allowing the validation of the simulations in the actual heat transfer conditions developed in the magnetic material.

\subsection{Fluid flow in the Rotary Thermomagnetic Motor}

The magnetic material in the rotary TMM proposed is formed into annular sections, in which each section presents a magnetic pole of the motor, being "activated" or not by the control of the flow of heat transfer fluids. The originally proposed working scheme of the motor developed was composed of three plates, as presented in Figure 33, and with that forming three poles of the motor. However an alternative design was adopted, in which six plates are used, this design can develop the original working scheme, by "activating" the plates in adjacent pairs, and also allows the exploration of different control designs. The construction with six plates also presents another advantage, the magnetic material plates are easier to fabricate, due to the smaller angular sections.

The design of the stator of the rotary magnetic motor is presented in Figure 56. This stator is composed of magnetic material plates supported by an aluminum structure, which 
holds the plates against the traction force induced by the permanent magnets arrangement, and also distributes the flow of the heat transfer fluid to the plates. The magnetic plates present a length of $100 \mathrm{~mm}$, thickness of $12 \mathrm{~mm}$, and form an arc of $49.4^{\circ}$. The plates must present internal channels for the flow of the heat transfer fluids, with the inlet and outlet of the channels on the right hand of the stator shown in Figure 56, due to the construction of the rotary TMM the stator is fixed to the support on the right side, while the support of the permanent magnets assembly is fixed to the rotor axis on the left side. The internal channels present an octagonal cross-section shape, with an equivalent hydraulic diameter of $8.36 \mathrm{~mm}$, and form a total heat transfer contact area of $18.21 \cdot 10^{-3} \mathrm{~m}^{2}$. The volume of magnetic material on the designed magnetic plates is $4.59 \cdot 10^{-5} \mathrm{~m}^{3}$.

Figure 56: The design of the support of the magnetic material plates acting as the stator of the rotary TMM, with the indication of the inlet and outlet connections of fluids.

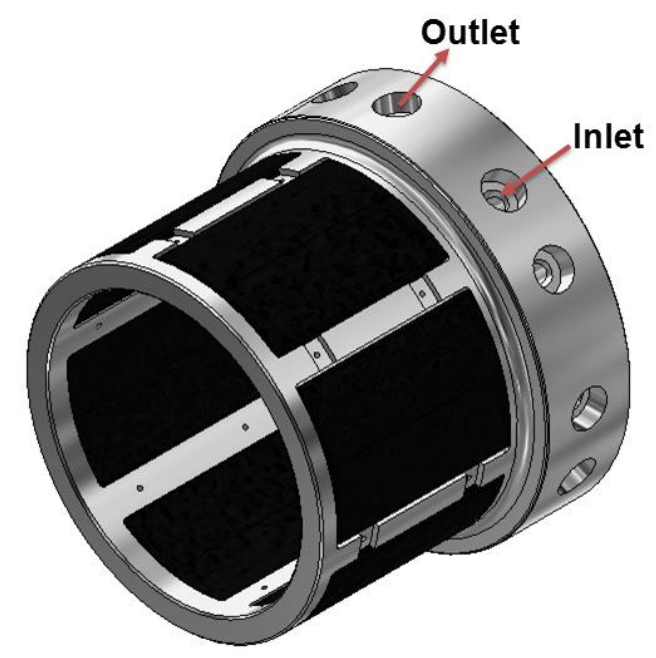

The heat transfer in the proposed rotary TMM is conducted through the flow of water, which is heated to the specified heat source temperature, between 343 and $353 \mathrm{~K}$, with water at room temperature $\left(T_{0}=298 \mathrm{~K}\right)$ being circulated to remove the heat from the plates, from which the magnetic material is "activated" by returning to the ferromagnetic state. The water flow through the motor is obtained using electrical pumps. To better understand the hydraulic characteristics of the system proposed an initial computational fluid dynamics analysis (CFD) was conducted.

The CFD analysis was performed using the software Ansys CFX, which presents a friendly user interface, with its solving process approved through Verification processes and 
Validated to a number of physical analysis (ANSYS, 2013). A section of the stator was used as the model for the CFD analysis conducted, as presented in Figure 57, this simplification allowed the solving process to be quicker, reducing computational costs, while still providing a good representation of the stator of the motor. The model also includes the two nylon connections that are used to fix the valves controlling the fluid flow, and also avoid the direct connection between the fluid and the aluminum support.

Figure 57: The model of the magnetic material plate and support structure used for the CFD simulations.

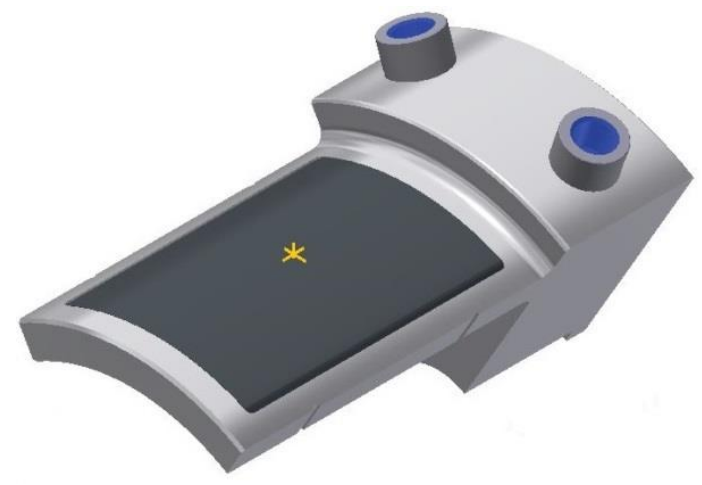

This first CFD analysis was conducted using the material data included in the Ansys CFX library. The mesh of the CFD problem was done using the control of inflation in the contact regions, which increases the quality of the results but at a higher computational cost, obtaining a mesh with a total of $2.32 \cdot 10^{6}$ elements. The parameter selected to evaluate the meshing process was the orthogonal quality, from which an average of 0.855 and standard deviation of 0.102 was obtained, from what the mesh can be classified as "very good", according to the software guidelines.

This CFD analysis is run in steady state condition, with the mass flow rate of water in the simulation set to $10 \mathrm{~g} \cdot \mathrm{s}^{-1}$. The fluid flow in the plates is expected to develop a turbulent flow. A number of models have been developed to approximate turbulence based on the Reynolds Average Navier-Stokes (RANS) equations. While some models have particular applications, the standard $\mathrm{k}-\epsilon$ model was adopted, since it provides a reasonable degree of confidence for an initial study. The implementation of the $k-\epsilon$ model for the software used is done using the intensity levels, in which the $0.1 \%$ level presents very low turbulence levels and $10 \%$ presents high levels of turbulence. As an initial approach, a low to medium turbulence level of $1 \%$ was used. 
As a result of the CFD analysis, the streamline of the fluid flow is presented in Figure 58. The water inlet is presented to the right lower side. From this analysis, the recirculating serpentine pattern of the fluid inside the internal channels of the plate is established, with the red regions indicating regions of higher stream velocity, and with that higher pressure loss.

Figure 58: The streamline of the fluid flow obtained from the CFD simulation, showing the recirculating serpentine pattern of the octagonal internal channels in the plate.

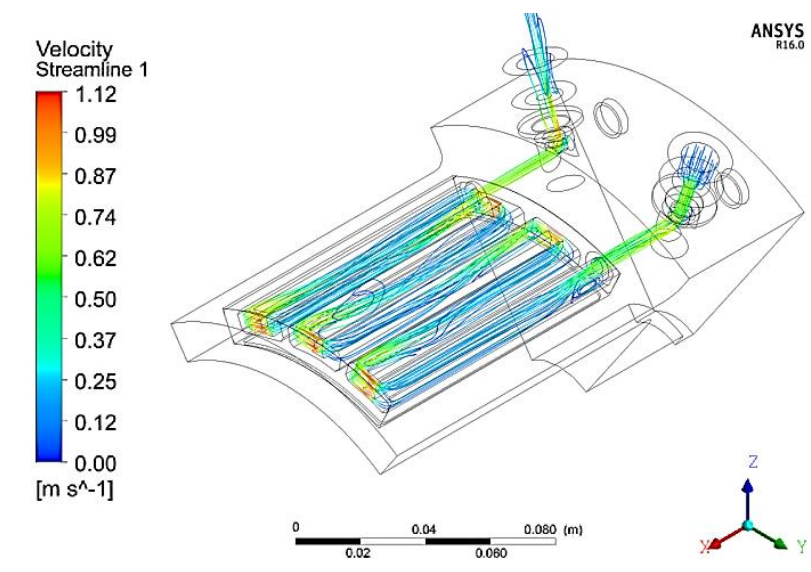

Important information for the fabrication of the stator of the rotary motor is derived from the simulation. In first place, the water volume contained inside the plate is determined to be of $4.3 \cdot 10^{-5} \mathrm{~m}^{3}$, comparable to the volume of the magnetic material plate, this volume presents a "dead fluid" which will be present inside the plate during the heating and cooling cycles. The second result is that a major contribution to the pressure loss occurs on the connections in the aluminum support and on the connection between the aluminum support and the plate of magnetic material. This connection presents a tight tolerance and can cause a high increase in the pressure loss of the system if a misalignment occurs.

The stator was then fabricated according to the design shown in Figure 56, as presented in Figure 59. The magnetic material plates are held in place using small fixing tabs bolted to the aluminum support. The plates are made using the $\mathrm{Gd}_{4.7} \mathrm{Nd}_{0.3} \mathrm{Si}_{4}$ material, agglomerated with $5 \%$ of Epoxy resin, in weight of the magnetic material, and compacted into the shape of the plates under high pressure. The process used to make the plates is described in Appendix 1. 
Figure 59: The stator of the rotary thermomagnetic motor, with the $\mathrm{Gd}_{4.7} \mathrm{Nd}_{0.3} \mathrm{Si}_{4}$ magnetic material plates mounted.

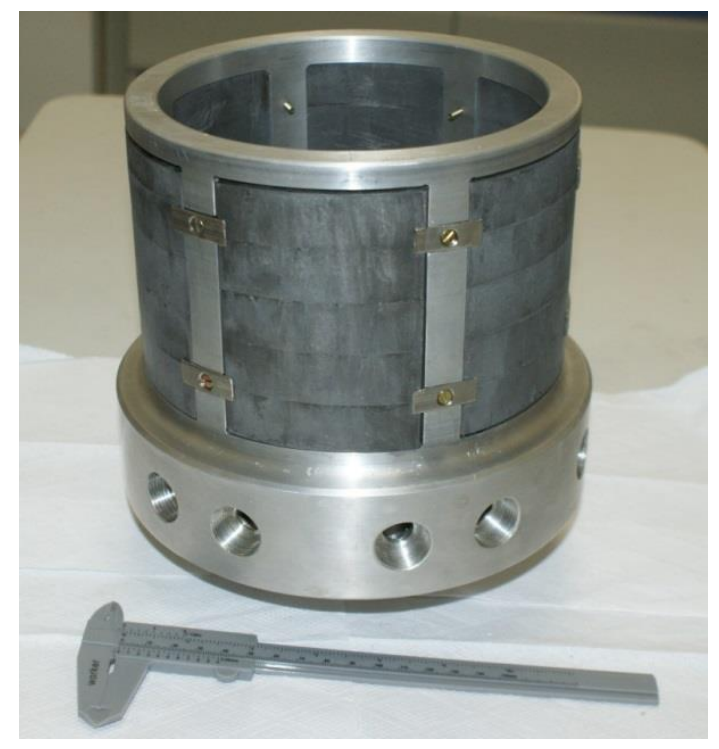

The magnetic plates are composed of five $20 \mathrm{~mm}$ sections, which are glued with the Epoxy resin to form the total length of $100 \mathrm{~mm}$, from which the seams of the sections can be seen in Figure 59. After being mounted to the aluminum support, the plates are then covered with a layer of Epoxy, which acts to reinforce the fixing of the plates and also to avoid the leaking of the water flowing through the internal channel of the plates and in the connection between the plates and the aluminum support.

As the circulation of water will determine the magnetization state of plates, the control of the fluid flow will act in the same manner as the control of the current in the coils of a stepper motor. The assembly of the fluid control valves and connections is complicated by the large number of connections, to each magnetic material plate two $2 / 3$ way solenoid valves must be used to direct the inlet and outlet flows, from and to the hot and cold water reservoirs. The valves selected to be used in the motor were the Bürkert, type 0124, 2/3 ways, with a polypropylene body, a total of 12 control valves are used in the motor. Each control valve is connected to fluid distributors, for the hot and cold water being pumped.

The software Inventor 2017, "Tube and Pipe" simulation environment, was used in order to allow an improved positioning of the valves, and facilitate the dimensioning of the hoses. Through the software, the positioning of the control valves and distributors were optimized to reduce the lengths of the hoses. The inlet valves were mounted directly to the 
stator of the motor, reducing the "dead fluid" volume on the inlet to the internal volume of the valves, avoiding a delay between the action of the valves and the circulation of the controlled fluid flow through the plates. The outlet control valves act directing the returning flow from the motor to the water reservoirs; due to lack of assembly space, these valves were positioned on the workbench supporting the motor. The software used calculates the length and positioning of the hoses, taking into account the minimum bend radius of the hose selected, for the PA-12 hoses with a diameter of $10 \mathrm{~mm}$ used in the motor, this radius is defined to be $50 \mathrm{~mm}$. The designed flow control system is presented in Figure 60, which can be compared to the picture of the built motor showed in Figure 37, in section 4.2.

Figure 60: The design of the hydraulic connections and hoses for the Rotary TMM, with the inlet valves directly mounted into the support of the magnetic plates, and the outlet valves fixed to the workbench.

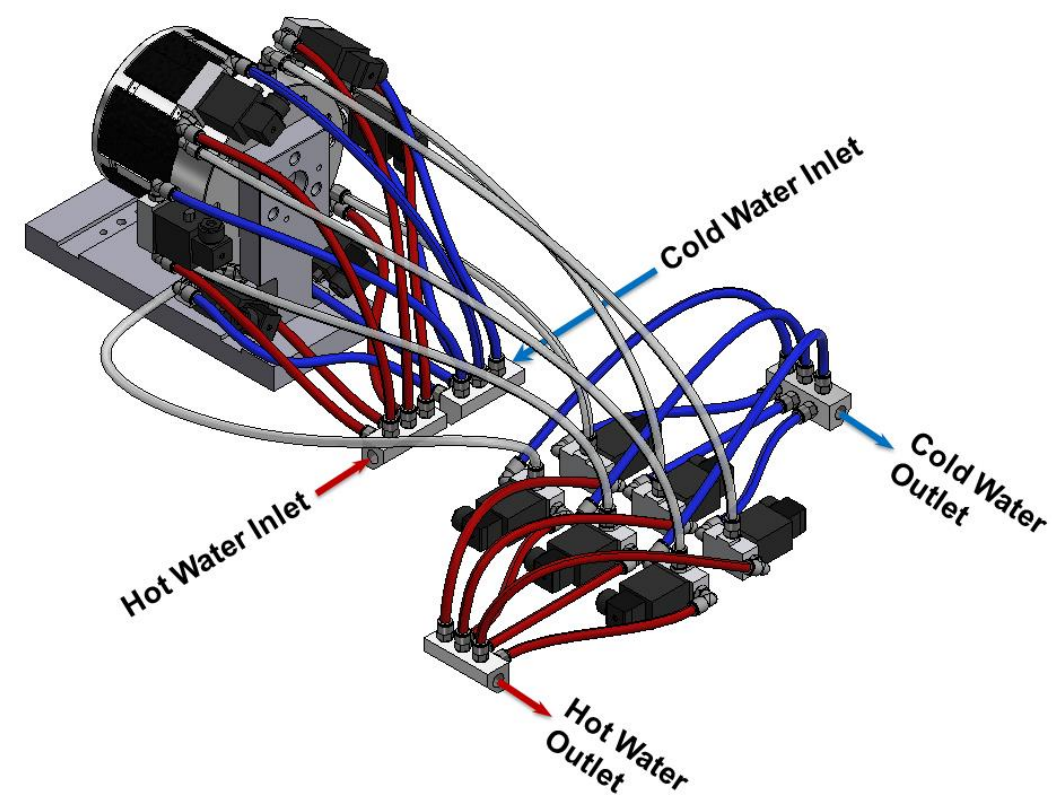

With the built stator of the thermomagnetic motor and flow distribution system, the hydraulic characteristics of the Rotary TMM can be determined and used to validate the CFD simulation of the motor. The first step then was the determination of the pressure loss in the built stator, and validation with the pressure loss obtained in the simulation. A differential pressure sensor NXP - MPX 2050 was used to measure the pressure loss on one plate. This sensor has a temperature compensation, a maximum reading of $50 \mathrm{kPa}$, and is provided with its sensitivity and precision characteristics, the sensor was tested and calibrated using a reference sensor MKS Baratron capacitance manometer. To measure the water flow in the plate analyzed an ultrasonic flow sensor was applied, the sensor used was the 
Flow Netix 107, which presents a resolution of $1.66 \cdot 10^{-8} \mathrm{~m}^{3} \cdot \mathrm{s}^{-1}$, and minimum accuracy of $\pm 5 \%$ of the read value.

To measure the pressure loss curve the mass flow through the plate was changed, using a manual needle valve, while the values read by the sensors were registered using the NI-USB-6363 data acquisition device, with an automatic experimental procedure developed through the LabVIEW software. The resulting curve is presented in Figure 61, with the uncertainty limits of the measurement obtained by composing the characteristic uncertainty limit of the sensors used with the statistical uncertainty from a series of measurements, using the MCM of propagation of uncertainties.

Taking the measured data as a reference, a CFD analysis of the pressure drop on the plates was then conducted by simulating increasing mass flow rates and obtaining the resulting pressure drop on the plate; this opposite approach is necessary to maintain the consistency with the analysis procedures previously shown. These simulations were done using the same mesh and parameters adopted for the first analysis.

The results show a lower pressure drop in the simulation, for the same mass flow rate, than in the experimental analysis, with that an adequation of the simulated model was necessary. By analyzing the built assembly, it was verified that there was a small misalignment between the inlet and outlet connections of the plate and the connections of the supporting aluminum structure, but it was not possible however to determine the exact amount of misalignment, as an alternative an obstruction in the outlet was used. Then by reducing the diameter of the outlet by step amounts a series of pressure loss curves could be compared to the measured data, as shown in Figure 61. From the analysis of the results, a reduction of $2.4 \mathrm{~mm}$ of the outlet diameter was capable of reproducing the obstruction caused by the misalignment in the mounting of the plate to the aluminum support. 
Figure 61: Pressure loss curve of one magnetic plate of the rotary TMM developed, showing the measured results and the simulation corrections.

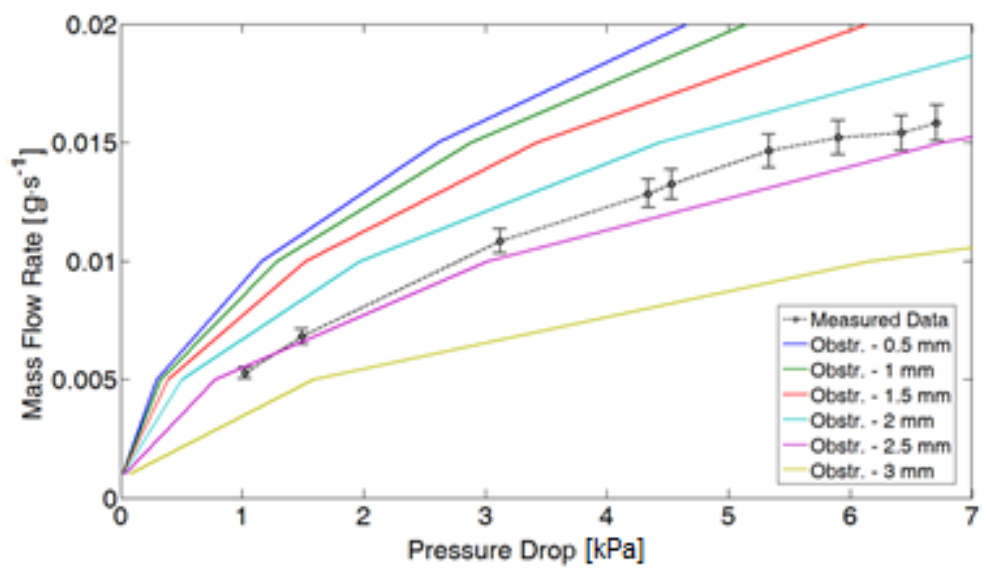

By adopting the adequate correction, the hydraulic behavior of the plates characterized experimentally was correctly described by the CFD simulation analysis. This validated analysis can consequently be used to model the heating of the magnetic material in the stator of the Rotary TMM, by the use of transient analysis and by setting the suitable properties of heat transfer of the material.

\subsection{Thermal cycling of the Magnetic Material}

To simulate the transient behavior of the temperature in the material, the timedependent analysis is started with both the magnetic material and the supporting aluminum plate at the temperature of $298 \mathrm{~K}$, and then hot water, at $343 \mathrm{~K}$, is circulated through the plate. This analysis is conducted with interval steps of 0.5 seconds. The magnetic material properties are set using first the measured heat capacity for the $\mathrm{Gd}_{4.7} \mathrm{Nd}_{0.3} \mathrm{Si}_{4}$, with the data presented in Figure 55. A second fundamental parameter to characterize the heat transfer in the material is its thermal conductivity $(k)$, this parameter was not readily available for the $\mathrm{Gd}_{4.7} \mathrm{Nd}_{0.3} \mathrm{Si}_{4}$ material and had to be determined experimentally, this spin-off experiment is described in Appendix 2. The data is shown in Figure 98.

Then, the thermal contact mode between the parts was set in the CFD simulation, in which the conservative energy mode was used for all the solid-solid contacts, as well as for the contacts between the fluid and the magnetic material, and the contacts between the fluid and the nylon support pieces. There is no direct contact between the fluid and the supporting aluminum places, though the support does exchange heat with the magnetic material plate. 
As only one-sixth of the actual stator is modeled in the simulation, an isothermal condition, with the temperature at the initial condition of $298 \mathrm{~K}$, is set for the two section walls of the aluminum support structure. With these changes and by applying the validated CFD model, using the same mesh parameters as in the previous simulations, a heat transfer modeling of the stator of the motor was obtained, as presented in Figure 62.

Figure 62: The temperature contours on the plate, obtained through the CFD simulation, after 15 seconds of heating.

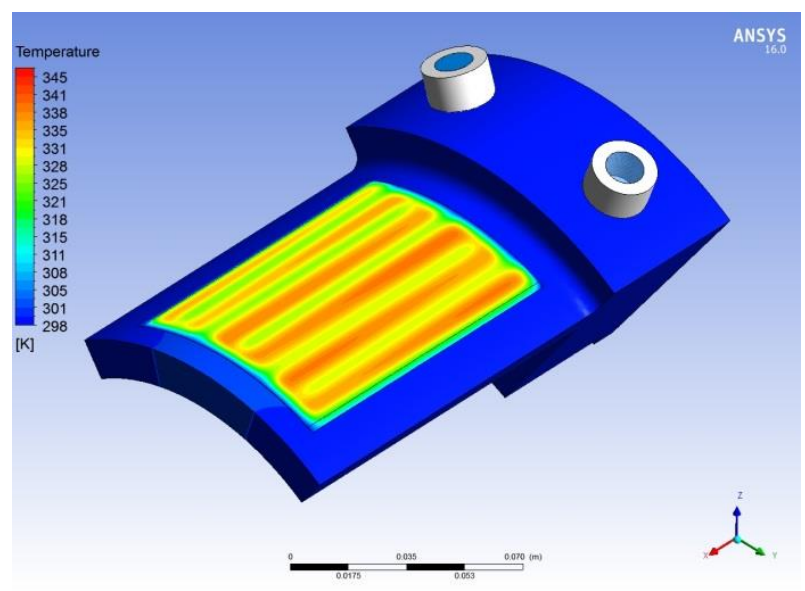

Following the adopted integrated approach of computational simulation and experimental analysis, the heat transfer in the stator of the Rotary TMM had to be characterized experimentally. This experiment was conducted using the mapping of the temperatures on the surface of the plate of magnetic material, through heating and cooling cycles. These cycles were developed on the same plate for which the pressure loss curve was measured and defined to characterize the behavior of the plates in the motor. Each heating and cooling cycle was defined to last for 30 seconds each, a period that, according to the heat transfer modeling done in the CFD simulation, would be large enough for the temperature of the plate to be higher than the transition temperature $\left(T_{C}\right)$ of the $\mathrm{Gd}_{4.7} \mathrm{Nd}_{0.3} \mathrm{Si}_{4}$ material $(327 \mathrm{~K})$.

The heating was done through the flow of water at the temperature of $343 \mathrm{~K}$, and after 30 seconds, the positions of the inlet valves were changed for the flow of water at room temperature $(\approx 298 \mathrm{~K})$. The temperature of the hot water reservoir was controlled during the experiments, using an electrical resistance and a temperature controller connected to a relay. The temperature of the water at the heat sink was kept at room temperature by heat loss to the environment. The water reservoirs had a volumetric capacity of $0.13 \mathrm{~m}^{3}$, which proved to be 
large enough to conduct a series of experiments without much change of the respective temperatures.

For the temperature mapping, the water flow rate was kept constant through the magnetic material plate, both for the heating and cooling cycles, using a manual needle valve to control the flow of the water from the hydraulic pumps. The established mass flow rate of water was kept on $11 \mathrm{~g} \cdot \mathrm{s}^{-1} \pm 0.7 \mathrm{~g} \cdot \mathrm{s}^{-1}$, measured using the ultrasonic volumetric flow sensor Flow Netix 107 and corrected for the density change with the varying temperature of the water. The uncertainty in the mass flow rate is composed by the characteristic uncertainty of the sensor used, and by the statistical variation in the registered flow during the experimental cycles, calculated using the MCM of propagation of uncertainties.

To approximate the simulated isothermal condition of the two section walls of the aluminum support structure, water at room temperature is circulated through the remaining five plates of the motor, and the support structure was verified to remain at room temperature by measuring its temperature. The temperature is measured in the inlet and outlet of the plate, verifying that the temperature of the flow was that of the reservoirs.

The mapping of the temperature developed on the surface of the magnetic plate is done using nine N-type thermocouples, models 401-310, NC class 1, from TC Direct, with PFA insulation, wires with $0.2 \mathrm{~mm}$ diameter, and nominal uncertainty of $\pm 1.5 \mathrm{~K}$. The same thermocouples are used to measure the inlet and outlet temperatures, as well as the temperature of the supporting aluminum structure. The cold-junction of all the thermocouples is connected to an isothermal aluminum plate, which has its temperature measured using the LM-35 precision temperature sensor, for cold-junction compensation. An analogic lowfrequency pass filter is connected to each thermocouple pair, to reduce the noise in the thermocouple measurements.

The data acquisition is made using the NI-USB-6363 DAQ device, with an automatic experimental procedure developed through the LabVIEW software. The data acquisition system reads the voltage of all the thermocouples, performs the cold-junction compensation, and conversion to temperature readings using look-up tables. The system also registers the measured mass flow rate, to perform the adjustment of the needle valves in the experiment, and automatically controls the inlet and outlet valves, actuating to maintain the cycling times 
established. The data acquisition rate for the experiments was of $1 \mathrm{kHz}$, with the total time per cycling of 60 seconds.

The fixing of the thermocouples to the surface was done with the thin layer of silver conductive paint, and each junction was then covered with Kapton tape for reinforcement. The experimental assembly is presented in Figure 63.

Figure 63: The experimental measurement of the temperature developed in the magnetic material plate. To the left side can be seen the nylon plug connections and the control valve.

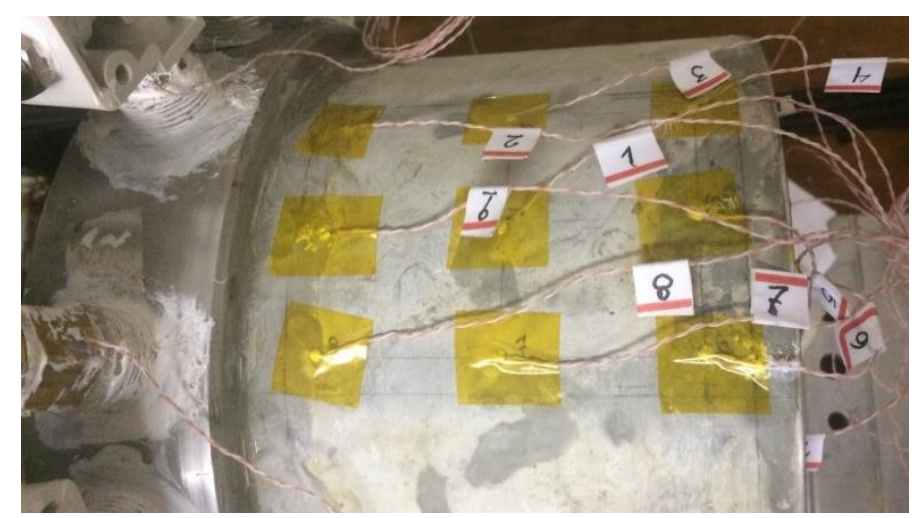

A total of 30 heating and cooling cycles were developed in the plate, divided into two separate sections, avoiding the possible excessive heating of the cold water reservoir. In the data analysis process, the initial runs are discarded, to maintain only the data from continuous cycling, and with that avoiding uncertainty on the initial conditions of the experiment. This way the total number of cycles analyzed was of 26 .

The uncertainty in the measurements of the temperatures in the plate was determined using a statistical analysis of the temperatures measured by each thermocouple in every time interval, for the 26 temperature cycles. This treatment allows the dynamic heating cycle to be statistically analyzed as a static phenomenon, with every time interval presenting an individual analysis with 26 data points. This method of analysis is adequate only for cyclical phenomena in which the time variance can be considered insignificant (BOX; HUNTER; HUNTER, 2005), as in the system the data acquisition rate is significantly higher than the heat transfer time constants, this approach was able to produce probability density functions for every temperature measured in each time step. With the use of the MCM, the statistical uncertainties were combined with the uncertainty provided by the nominal precision of the thermocouples used. 
The graph presented in Figure 64 summarizes the results obtained in the experimental temperature mapping, in it the reference point assumed is the temperature at the center of the plate, the thermocouple number 1 in Figure 63, and indicated by a yellow mark in the model shown in Figure 57. Only one percent of the data points collected in the experiments are shown in the graph, to avoid excessive cluttering, and the error bars shown in the graph present the $95 \%$ confidence bounds.

Figure 64: The temperature cycle developed by a magnetic plate of the Rotary TMM, the reference point represents the temperature at the center of the outer surface of the magnetic plate.

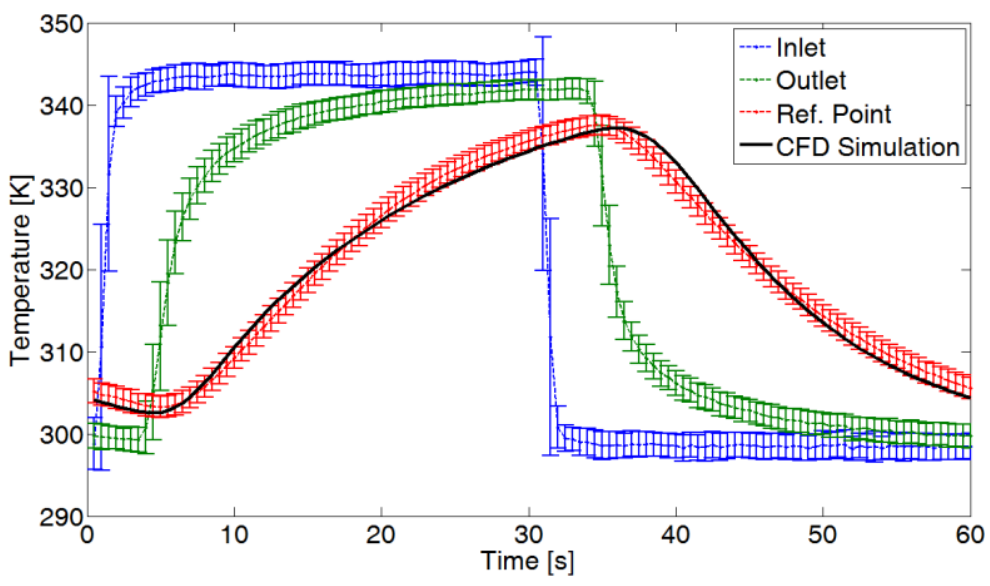

The CFD heat transferring model was then compared to the measured data; the initial results demonstrated slower heating of the magnetic plate in the experiments than in the simulation. The first correction of the CFD model was to use the fitting of the measured inlet temperature as input for the inlet temperature in the simulation. Initially, the model assumed a step function of the inlet temperature, while the experimental data shows a small onset time. This alteration of the model showed a better approximation between the simulated heating of the plate and the experimental data.

The second correction was the definition of thermal contact resistance (TCR) between the plate of magnetic material and the water flowing through the internal channels. The addition of a thin contact resistance is a better representation of the physical problem modeled, since there may occur the accumulation of a thin layer of oxide material in the contact region. With that, the CFD modeled heat transfer was able to adequately represent the temperature of the magnetic material during the heat cycling, as presented in the graph shown in Figure 64. 
From the analyses of the difference in the temperature between the inlet and outlet streams of the plate, it is possible to determine an amount of heat that is lost by the mass flow rate of water to the heating of the magnetic material plate in the assembly, by applying equation 6.1, modified to:

$$
Q_{w}=\int_{t_{0}}^{t_{f}} \dot{m}_{w} \cdot c_{w\left(T_{\text {in }}\right)} \cdot\left[T_{\text {in }}(t)-T_{\text {out }}(t)\right] d t
$$

In which $Q_{w}$ is the amount of heat lost by the stream flow (J), the mass flow rate of water $\left(\mathrm{kg} \cdot \mathrm{s}^{-1}\right)$ is given by $\dot{m}_{w}$, the heat capacity of water $\left(\mathrm{J} \cdot \mathrm{g}^{-1} \cdot \mathrm{K}^{-1}\right)$, which depends on the temperature, is given by $c_{w}, T_{\text {in }}$ and $T_{\text {out }}$ present the temperature of the flow on the inlet and outlet, and the time of heat transfer, given by $t_{f}$ after the start of the evaluation $t_{0}$. Using the mass flow rate of the experiment and the specific heat of the water in the inlet, the amount of energy lost during a heating cycle is of 30 seconds is $424.6 \mathrm{~kJ}$.

By taking the temperature at the reference point $\left(T_{r e f}\right)$, from Figure 64, as a representation of the mean temperature of the material, which was observed to be a reasonable assumption from the mapping of the temperature on the surface of the plate, then by applying equation 6.1 to the sample of magnetic material we get:

$$
Q_{\text {mat }}=m_{\text {mat }} \cdot c_{\left(T_{\text {ref }}\right)} \cdot \int_{t_{0}}^{t_{f}} T_{\text {ref }} d t
$$

From which $Q_{\text {mat }}$ is the amount of heat gained by the material in the process; $c$ is the specific heat of the material, obtained from the data shown in Figure 55 for a zero applied magnetic field; and $\mathrm{m}_{\text {mat }}$ being the mass of magnetic material of the plate, of $208 \mathrm{~g}$. This way the amount of heat received by the plate during a heating cycle of 30 seconds is determined to be $93.3 \mathrm{~kJ}$. Although the values of heat calculated are approximations, these values can be used to estimate the efficiency of heat transfer in the system built, determined to be in the order of $22 \%$.

Using the experimentally validated CFD simulation, additional and important information can be obtained about the heat transfer process in the magnetic plates of the Rotary TMM. As the analysis of the volume of magnetic material that is heated above the critical temperature of the $\mathrm{Gd}_{4.7} \mathrm{Nd}_{0.3} \mathrm{Si}_{4}$ material, of $327 \mathrm{~K}$, which gives a reasonable expectation of the use factor for the conversion of energy through the thermomagnetic effect 
in the system, this relative volume of transitioned material is presented in Figure 65. Showing that, using the heating cycle modeled, it will take up to 20 seconds for the major part of the magnetic material plate to change into a paramagnetic state. The data also shows that, even after 30 seconds, there still is almost one-quarter of the plate that is at a temperature below $\mathrm{T}_{\mathrm{C}}$

Figure 65: Relative volume of the magnetic material plate that is below the critical temperature $\left(\mathrm{T}_{\mathrm{C}}\right)$ of the $\mathrm{Gd}_{4.7} \mathrm{Nd}_{0.3} \mathrm{Si}_{4}$ material of $327 \mathrm{~K}$, obtained using the experimentally validated CFD simulation.

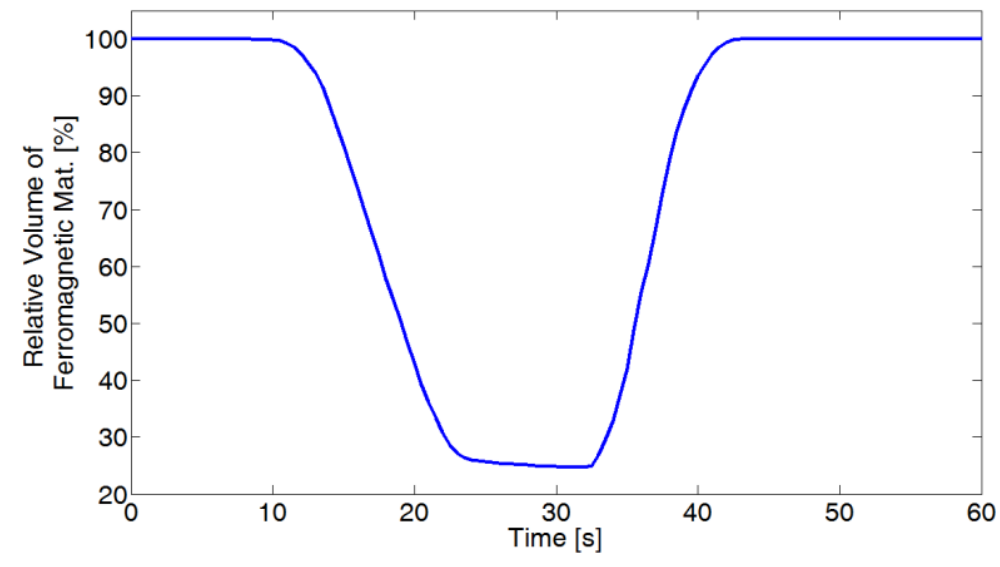




\section{THE THERMOMAGNETIC MOTOR DYNAMICS}

The principle of energy conversion for the thermomagnetic motors is based on the interaction between a magnetic material with varying magnetization, due to a change in its temperature, and an applied magnetic field gradient, producing mechanical work from the heat energy received by the material. This chapter explores the production of work obtained from the motors studied, analyzing the dynamic behavior of these systems, and also presenting a discussion on the logic of control to be developed.

Initially, the idealized TMM is experimentally tested, aiming to obtain the work output curves for the reference material Gadolinium (Gd) and then the $\mathrm{Gd}_{4.7} \mathrm{Nd}_{0.3} \mathrm{Si}_{4}$ compound. The magnetic forces obtained from the experimental test stand are then used to validate the magnetostatic simulations for these materials, with the experimental procedure and the uncertainties on the measured data presented. Then an application of the work output curves is explored.

For the Rotary TMM developed, the torque output is obtained using the validated magnetostatic simulation, from which the torque output is then used to simulate the dynamic motion of a rotor of the motor. The results of the dynamic simulation are then compared to the dynamic behavior of the built motor, using the angular position with respect to time as a parameter to compare the data.

\subsection{The magnetization work for the idealized TMM}

The test stand is proposed to represent the functionality of a thermomagnetic motor in an idealized manner, as described in section 4.1. It was applied to measure the magnetic force provided by the interaction of the magnetic field gradient produced by a permanent magnets assembly and two magnetic materials, $\mathrm{Gd}$ and the $\mathrm{Gd}_{4.7} \mathrm{Nd}_{0.3} \mathrm{Si}_{4}$ compound, varying the distance of the magnets from the material, and the temperature of the material. The resulting curves can then be used to calculate the available magnetic work present in a thermomagnetic motor operating between two measured temperatures and with a given displacement.

The first step in the measurements was the calibration of the spring force produced by the linear flexure bearing, shown in Figure 29, the use of this type of support avoid the 
influence of the random error caused by friction on the measured force, but introduces a systematic error due to the deformation of the plates on the bearing. As the spring force is repeatable, and in essence a function only of the deformation, it can be subtracted from the measured force in the experiments, to obtain the magnetic force on the material. The procedure is done by assembling the complete test stand, removing only the magnetic material, from which a curve of the measured force, produced by the deformation of the flexure bearing, is obtained in function of the position of the magnets support.

The resulting calibration curve of the flexure bearing is presented in Figure 66.a. Note that the force changes direction as the position crosses the point of zero deformation on the curve. Then using the calibrated curve the measurement is done a second time, as shown in Figure 66.b, this curve shows the resulting random error in the measurement of the force, this error is close to the nominal uncertainty of the load cell used, of $\pm 0.05 \mathrm{~N}$. To provide a complete evaluation of the uncertainty in the measured data, the calibrated curve is run a series of times, allowing a statistically significant analysis of the random error observed. Then the expanded uncertainty in the measurement forces was determined by combining the normal distribution of the random error with the uniform distribution given by the nominal uncertainty of the load cell, using the MCM of propagation of uncertainties. The resulting expanded uncertainty is determined to present a standard deviation of $0.035 \mathrm{~N}$, and with that, the $95 \%$ confidence bounds on the measured force are determined to be of $\pm 0.07 \mathrm{~N}$.

Figure 66: The calibration curve of the linear flexure bearing (a), and the resulting calibrated measured force for the stand without any magnetic material (b).

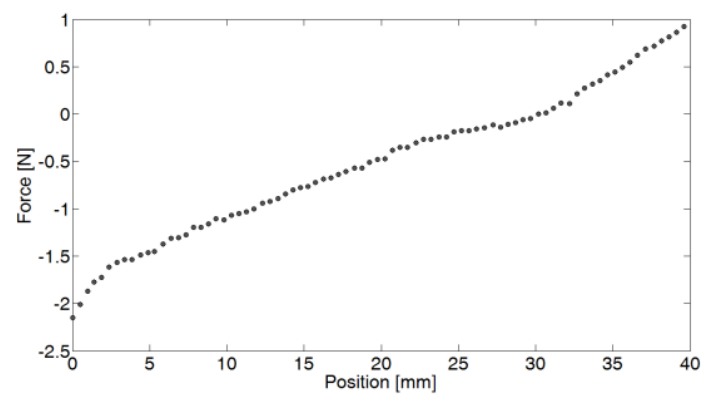

(a)

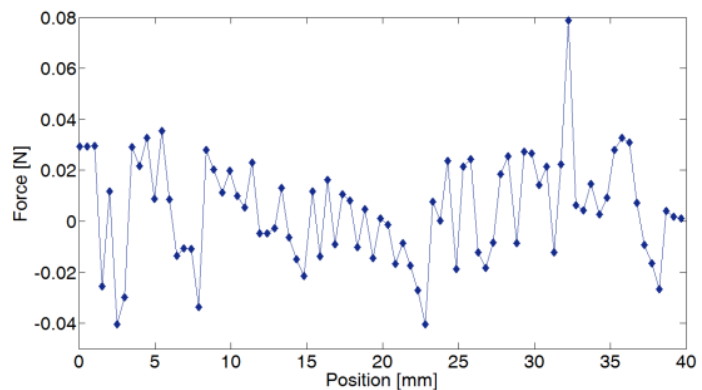

(b)

After the calibration of the flexure bearing, the magnetic material was mounted into the sample holder, shown in Figure 29.b, which holds the sample against the face of the Peltier element used for the temperature control. The control is done based on the average values of the temperatures measured on the face of the sample in contact with the Peltier 
element and on the opposing face, as indicated in Figure 29.b. The control was implemented using the National Instruments NI-USB-6363 DAQ device, with a PID architecture implemented in the LabVIEW software, after the calibration of the controller the average temperature of the magnetic material could be stably adjusted within $1.0 \pm 0.5 \mathrm{~K}$.

The permanent magnets are then positioned in contact with the external surface of the sample holder, for which the zero position is registered. Then the average temperature of the magnetic materials is controlled to the desired set-point; after the temperature is stabilized to a value within $\pm 0.5 \mathrm{~K}$ of the set-point, the magnetic force is registered into the data acquisition system. Next, the permanent magnets are displaced from the sample holder, for an established step distance of $0.5 \mathrm{~mm}$, which is verified using the linear encoder in the system, with a resolution of $0.07 \pm 0.02 \mathrm{~mm}$. The temperature of the sample is again verified to be stable with the desired temperature set-point before the induced magnetic force measured is registered, reinitiating the cycle, until the magnetic circuit reaches the maximum distance of $40 \mathrm{~mm}$. After this last step, the magnets are returned to the zero position, at which point the temperature set-point is changed and the cycle restarted.

It is important to notice that the measurement of the magnetic force is realized in a static manner, i.e., the magnetic force is registered only when the linear actuator is completely stopped, by using this static measurement method, the system is not influenced by the eddy currents that may occur due to a fast-changing magnetic field. Another point of notice is that, to determine the actual temperature developed on the material it is necessary to consider the composed uncertainties of the thermocouples used (models 401-310, NC class 1, from TC Direct) and the measurement system. However, the automatic experimental procedure developed works to constantly keep the mean temperature of the material at the desired set-point, and only allows the force to be registered if the measured value of temperature is kept within the controller stability criteria of $\pm 0.5 \mathrm{~K}$. Therefore, for simplicity, the temperatures of the material reported for these experiments consider the controlled set-point as the temperature of the sample.

The first material analyzed using this test stand, and measurement system was Gadolinium (Gd), using a cylinder-shaped sample with a diameter of $15.2 \mathrm{~mm}$ and thickness of $4.12 \mathrm{~mm}$, with a mass of 5,577 $\mathrm{g}$. The measurements were realized from a starting point of 
$278 \mathrm{~K}$ and run up to a temperature of $328 \mathrm{~K}$, with increments of $2.5 \mathrm{~K}$, the resulting magnetic force curves as a function of the material position are presented in Figure 67.

Figure 67: The magnetic force between the permanent magnets arrangement and a Gadolinium sample, with a mass of $5,577 \mathrm{~g}$, at different temperatures.

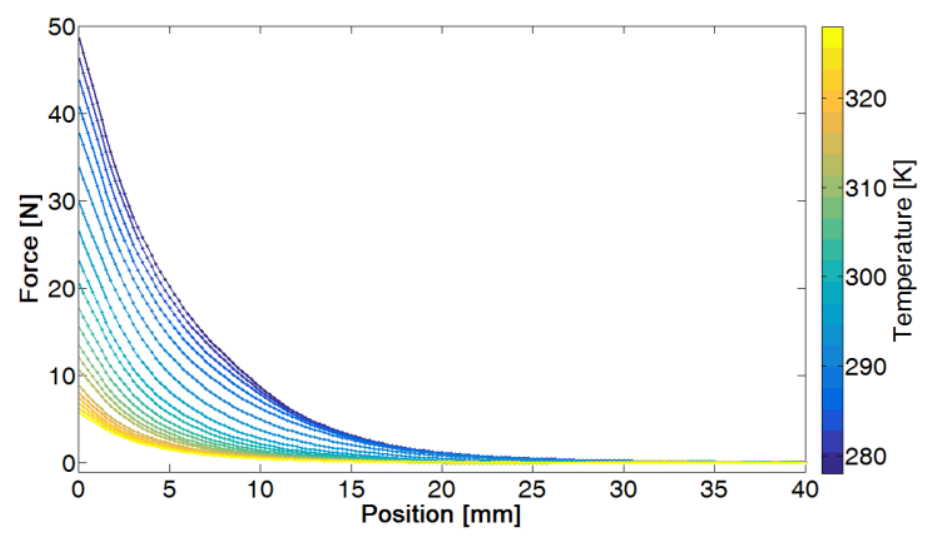

Since the Gadolinium is a second-order material, the measured magnetic force is reduced by a small amount with the increase of the temperature, until the critical temperature $\left(T_{C}\right)$ of $293 \mathrm{~K}$ is reached, above this temperature a more abrupt reduction in the force is observed, until the temperature of the material is increased above the total transition, at each point the force measured is reduced to a minimum value at very high temperatures. As presented in Figure 67, after a displacement of the magnets of $20 \mathrm{~mm}$, the magnetic force is reduced to almost zero.

The same analysis was then repeated for the $\mathrm{Gd}_{4.7} \mathrm{Nd}_{0.3} \mathrm{Si}_{4}$ compound, using a cylinder-shaped sample with a diameter of $15.1 \mathrm{~mm}$ and a thickness of $2.2 \mathrm{~mm}$, with a mass of $1.844 \mathrm{~g}$. The measurements were realized from a starting point of $308 \mathrm{~K}$ and ran up to a temperature of $338 \mathrm{~K}$, with increments of $4 \mathrm{~K}$, the difference in temperatures for this experiment was due to the higher $T_{C}$ of this compound. The resulting magnetic force curves as a function of the material position are presented in Figure 68, from which it is clear that the material presents a similar second-order behavior as the $\mathrm{Gd}$. 
Figure 68: The magnetic force between the permanent magnets arrangement and a sample of the $\mathrm{Gd}_{4.7} \mathrm{Nd}_{0.3} \mathrm{Si}_{4}$ compound, with a mass of $1.844 \mathrm{~g}$, at different temperatures.

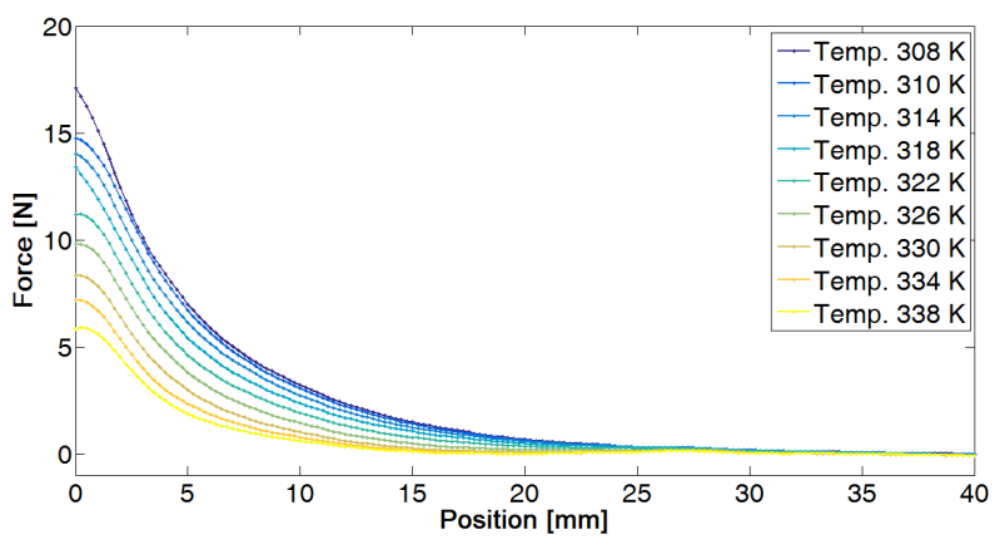

The comparison between the two measurements indicate that a higher force is obtained by using Gadolinium, with a maximum measured force of $48.6 \mathrm{~N}$, than by using the $\mathrm{Gd}_{4.7} \mathrm{Nd}_{0.3} \mathrm{Si}_{4}$ compound, which produced a maximum force of $17.09 \mathrm{~N}$. However it is important to notice that the samples have different masses, by dividing the maximum force by the mass of the samples the force densities will be of $8.71 \cdot 10^{6} \mathrm{~N} / \mathrm{g}$ and $9.27 \cdot 10^{6} \mathrm{~N} / \mathrm{g}$, respectively for the $\mathrm{Gd}$ and the $\mathrm{Gd}_{4.7} \mathrm{Nd}_{0.3} \mathrm{Si}_{4}$ compound. Though this force density must not be viewed as an intrinsic property of the material, but rather as a property developed by the application of the material in the measurement system, since, as can be derived from equation 3.1, the intensity of the force will also depend on the applied field gradient.

Using the magnetic force obtained in the experiments, a surface plot of the force as a function of the position and temperature of the material is obtained. This surface represents the behavior of the system built on an idealized thermomagnetic motor, as is presented in Figure 69, for the Gadolinium. One caveat from this curve is that it only represents an ideal system that is operating with the applied magnetic field gradient developed by the permanent magnets assembly used since different gradients will present different behaviors. 
Figure 69: The magnetic force curve for the Gadolinium for an idealized thermomagnetic motor.

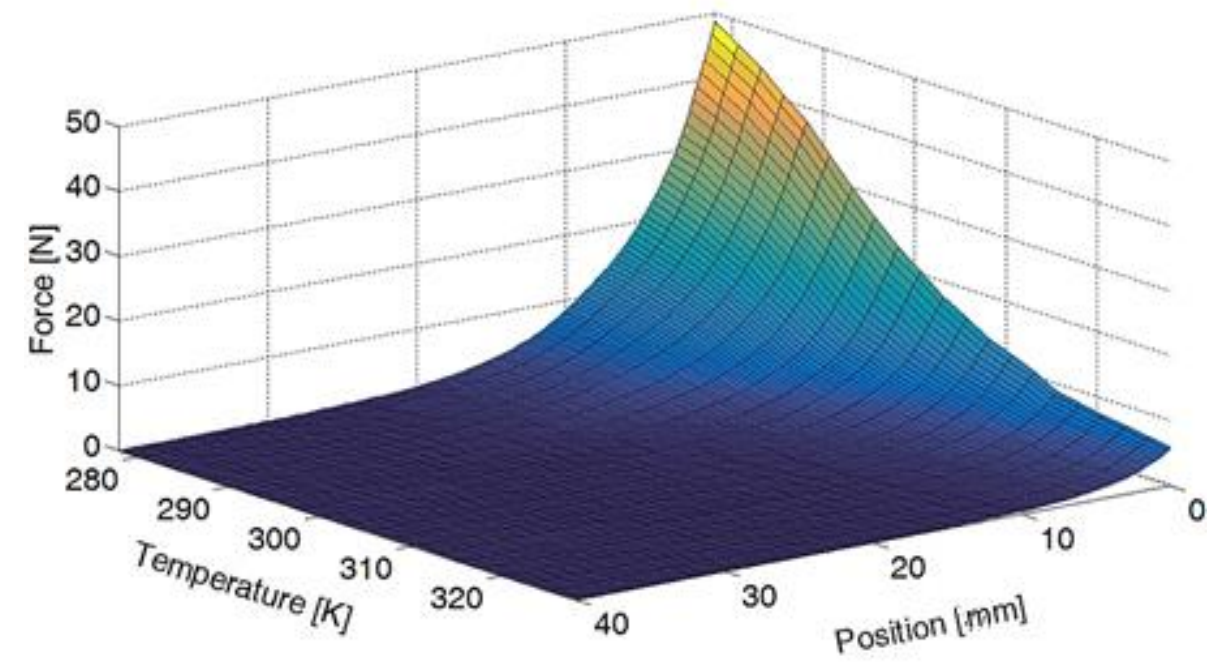

From the plot shown in Figure 69, it is possible to observe the thermomagnetic effect, by fixing a position and increasing the temperature of the material. It is also possible to identify the change in the force with relative movement of the material for a given temperature. From which the cycle of the idealized thermomagnetic motor could be described, that is a motor operating under the described relative position change and temperature variation, without taking into account any losses by friction or irreversibilities in the heat transfer.

Another interesting interpretation from these data is made possible by integrating the forces concerning a differential relative position change. Then by dividing this value by the mass of the Gadolinium sample, obtain the specific work of magnetization developed by the system. The linear displacements are correlated to the measured applied magnetic field gradient produced by the permanent magnets arrangement (shown in Figure 48). With that obtaining a 3D graph surface of the specific magnetization work of the material, as a function of its temperature and the applied magnetic field change from a zero field region, as presented in Figure 70. 
Figure 70: The measured magnetization work of Gadolinium as a function of its temperature and the applied magnetic field change from a zero field region.

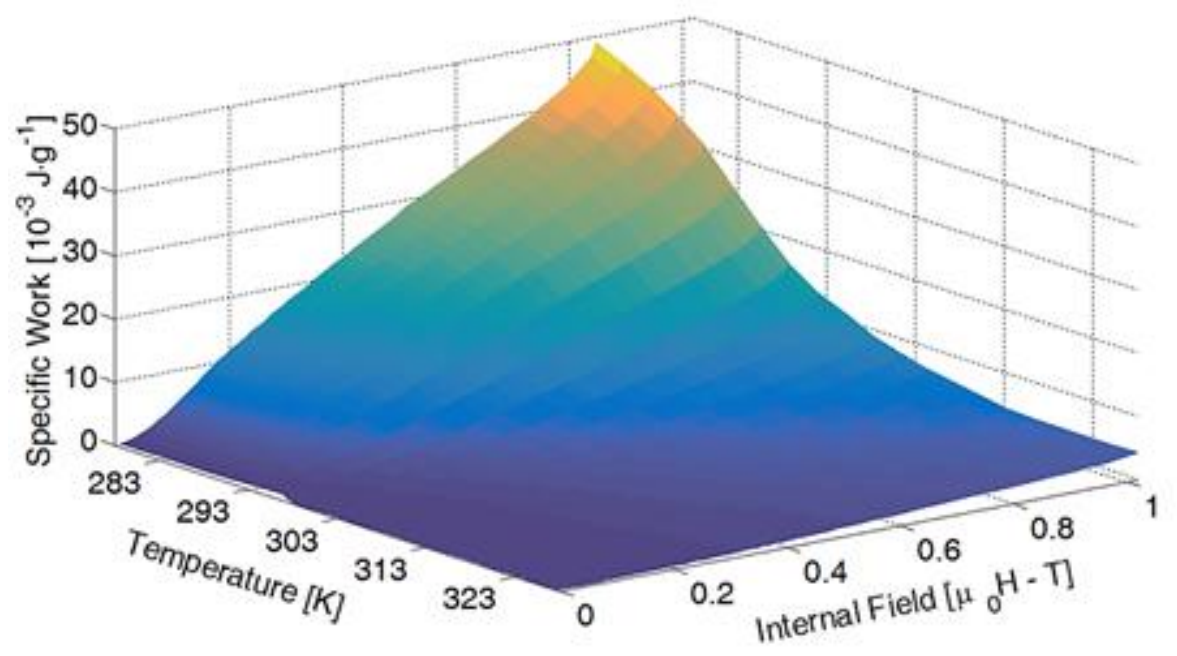

The curves presented in this study should be correct regardless of the magnetization direction. That is, the specific work that is obtained from bringing a sample at a given temperature from zero field into a higher field is reversible. This way, the graphs can be used to calculate the available work in the theoretical, idealized thermomagnetic motor. The magnetization work curve for Gadolinium, shown in Figure 70, can be used to determine the amount of work that is produced by the magnetic interaction when bringing a Gd sample at $278 \mathrm{~K}$ from zero field into a $1 \mathrm{~T}$ region is $45 \mathrm{~kJ} \cdot \mathrm{g}^{-1}$. If the sample is then heated to $303 \mathrm{~K}$, the amount of work that will be necessary to remove the material from the $1 \mathrm{~T}$ region into zero field will be $11 \mathrm{~kJ}^{-1}$, resulting in a theoretical maximum available work for this field gradient of $34 \mathrm{~kJ} \cdot \mathrm{g}^{-1}$. As this system does not take into account any losses, the available work in a real system operating in the same conditions should be expected to be smaller.

Another interesting insight obtained from the analysis of the magnetization work curve of Gadolinium is that the temperature change of the material acts in the same manner as the electric current in a stepper motor. Given a fixed applied magnetic field change, in Figure 70 the amount of the specific work produced will vary according to the temperature change. Although the temperature cycle developed by the material is not directly controlled on a real thermomagnetic motor, but rather a consequence of the heat transfer with the heat sources, the material will demand a higher temperature variation in order for its work output to be increased. If a constant heat transfer rate is applied, the TMM will develop a lower 
frequency of operation for higher work output demands, in the same manner as, if a constant current is applied to a stepper motor its rotational speed will be reduced for a higher work output.

\subsection{Validation of the simulated Magnetic Forces}

The magnetic forces measured with the test stand for the idealized TMM also present a valuable source as reference curves for the validation of the 3D magnetostatic simulations, modeling the interaction between the permanent magnets assemblies and the magnetic materials used in the motors. The magnetic field produced by the magnets assemblies was adequately validated for both the idealized TMM test stand, and the Rotary TMM, as presented in sections 5.1.2 and 5.2. Despite this, the introduction of the magnetic materials used in the motors present a significant modification of the original problem, since the computational solver must now consider the force induced in the material and the change in the induced magnetic field lines caused by the presence of the material. Therefore the force experiments were simulated and compared to the data obtained experimentally.

The model used for the 3D magnetostatic simulations, presented in Figure 71, will be the permanent magnets assembly that was used in the test stand to characterize the idealized TMM, with a magnetic material of cylindrical shape and dimensions equal to the samples used in force measurement experiments, shown in the previous section. The permanent magnets are the same $\mathrm{NdFeB}$ from which the magnetic field was already validated, as is the soft steel material SAE 1010, which the magnetic properties are included in the library of the Ansys Maxwell software. On the other hand, the properties of the magnetic materials used had to be manually introduced in the simulation. The magnetization curves of the Gadolinium material can be found in the literature, as presented in Figure 22, as for the $\mathrm{Gd}_{4.7} \mathrm{Nd}_{0.3} \mathrm{Si}_{4}$ compound it had to be determined experimentally, from which the data presented in Figure 36 was used in the simulations. Naturally, the magnetization curves depend on the temperature of the material, for this validation the temperature of $278 \mathrm{~K}$ was assigned to the Gadolinium sample, and $314 \mathrm{~K}$ to the $\mathrm{Gd}_{4.7} \mathrm{Nd}_{0.3} \mathrm{Si}_{4}$ compound. 
Figure 71: Model of the permanent magnets assembly with the magnetic material, used to validate the magnetic force obtained through the 3D magnetostatic simulations.

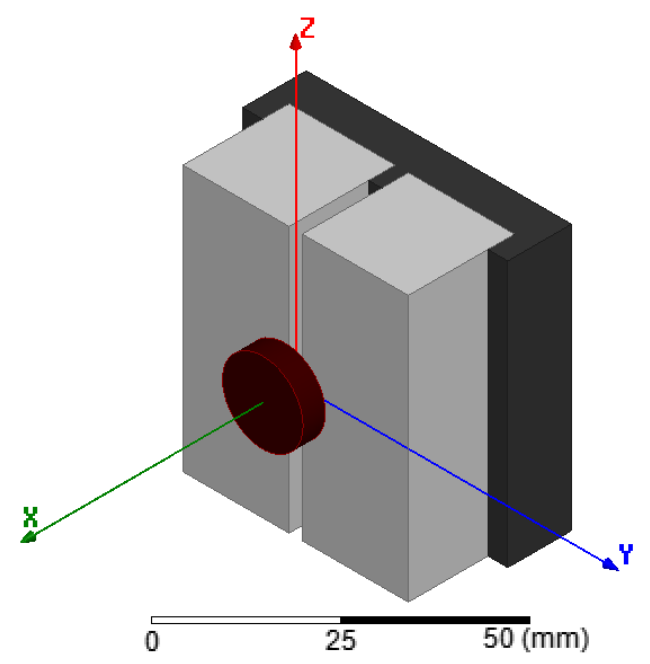

In order to model the change in the magnetic force with the displacement of the permanent magnets, the material was initially placed in the zero position and then dislocated in the $\mathrm{X}$ direction, Figure 71 , with step increments of $1 \mathrm{~mm}$. The magnetostatic simulation was done using a mesh refinement of maximum length, with a maximum allowable mesh edge length of $1 \mathrm{~mm}$ to the solid elements and of $10 \mathrm{~mm}$ to the surrounding atmosphere. The convergence was controlled by the percentage change of the energy error, using a convergence parameter of $0.01 \%$. As these simulations involved the movement of the sample, the meshing process was run for every time step, increasing the computational costs of the analysis.

One important point of notice, for the comparison between the simulation results and the measured data, is that the displacement of the magnetic material in the simulations starts from the material in contact with the permanent magnets assembly. However, this condition does not represent the actual start of the measurements in the experiment, since the displacement of the magnets is initiated from the contact with the sample holder. To correct this difference, the starting position of the measured data was dislocated by a distance of $2 \mathrm{~mm}$, with no other changes made to measured values. The results comparing the simulated results to the measured magnetic force of the $\mathrm{Gd}$ and $\mathrm{Gd}_{4.7} \mathrm{Nd}_{0.3} \mathrm{Si}_{4}$ compound are presented in Figure 72. 
Figure 72: Comparison between the magnetic force simulated and that obtained using the 3D magnetostatic simulation, for the Gadolinium at $278 \mathrm{~K}$ (a) and the $\mathrm{Gd}_{4.7} \mathrm{Nd}_{0.3} \mathrm{Si}_{4}$ compound at $314 \mathrm{~K}$ (b)

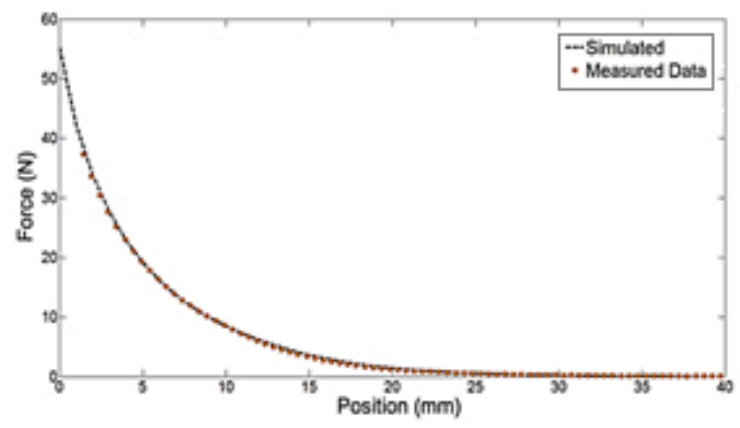

(a)

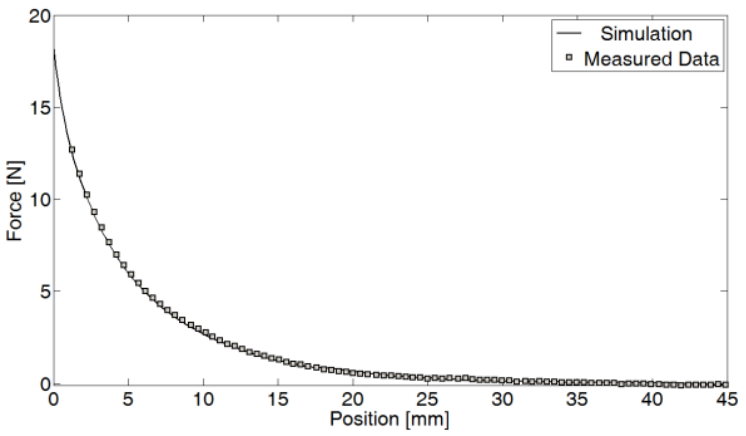

(b)

The results presented in Figure 72 show an excellent agreement between the simulation results and the measured data, with the overlap of the results. Although this could already indicate the validity of the simulated model, a more careful analysis is necessary due to the difference in the scale of the measured maximum force and the possible errors occurring in the simulation. Therefore, to better analyze the approximation between the simulated results and the measured data, a linear regression was conducted, for the Gadolinium experiment (Figure 72.a), using the measured magnetic force as independent data, the result of which is presented in Figure 73.a. The regression presented a coefficient of determination $\left(\mathrm{R}^{2}\right)$ of 0.9996 , with a root-mean-squared error (RMSE) of $0.1690 \mathrm{~N}$, the residuals from the regression are then plotted as shown in Figure 73.b.

Figure 73: Analysis of the approximation between the simulated results and the measured data, in (a) the linear regression of the data, and in (b) the plotting of the residual errors from the regression.

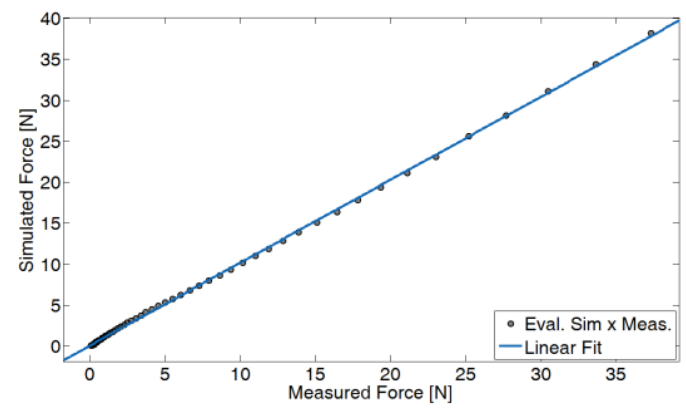

(a)

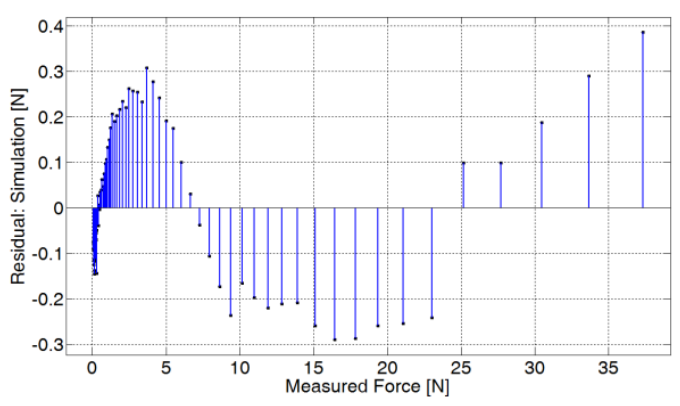

(b)

The analysis of the residuals shows a good agreement between the simulated magnetic force and that obtained experimentally for the Gadolinium sample since the scale of the residuals is smaller than $1 \%$ of the force measured. With that, it can be concluded that the 
magnetic force obtained through the $3 \mathrm{D}$ magnetostatic analysis approach used is validated experimentally.

\subsection{The power output of the Rotary Thermomagnetic Motor}

The output of the Rotary TMM developed is based on the interaction between the magnetic field produced by the permanent magnets assembly presented in section 6.4, and the magnetic material plates that are being cyclically heated and cooled. The way in which the magnetic plates are either heated, therefore changing their magnetic states from ferromagnetic to paramagnetic, or cooled, changing from paramagnetic to ferromagnetic, defines the configuration of control of the motor, similarly to the manner in which the electric current acts on the coils of a stepper motor, where the plates cooled to the ferromagnetic state represent the active coils.

The design initially proposed for the Rotary TMM conceived the use of three magnetic plates, working in the scheme presented in Figure 33. Though as explained in section 6.1, an alternative six plates construction was adopted in the developed Rotary TMM, this configuration allows the system to operate both using the originally proposed working scheme, with the plates being heated and cooled in adjacent pairs, and also an alternative controlling scheme in which each plate is "activated" individually by the cooling of the material. The new design also allows the motor to operate as a three poles electric motor, with two opposing plates being activated simultaneously. These three different configurations of control are presented in Figure 74, where the plates being cooled are represented by the blue color, and the plates heated presented in red. In all the designs the movement of the rotor occurs in a clockwise direction, at which point the flow control system redirects the cooling water to the next set of plates. 
Figure 74: Section view of the Rotary TMM, presenting three possible configurations of control, in (a) the three plates approach proposed initially, in (b) the six plates redesign, and in (c) the three poles configuration.

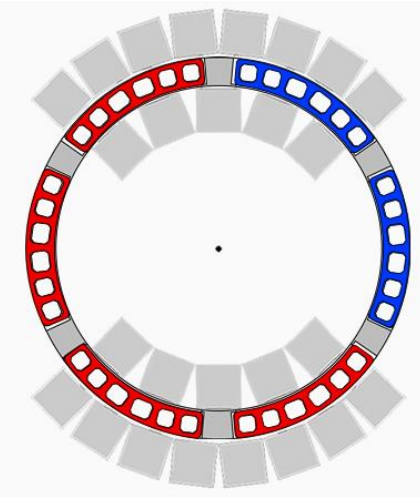

(a)

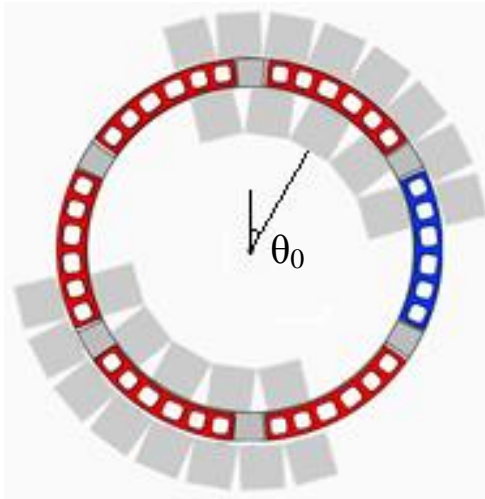

(b)

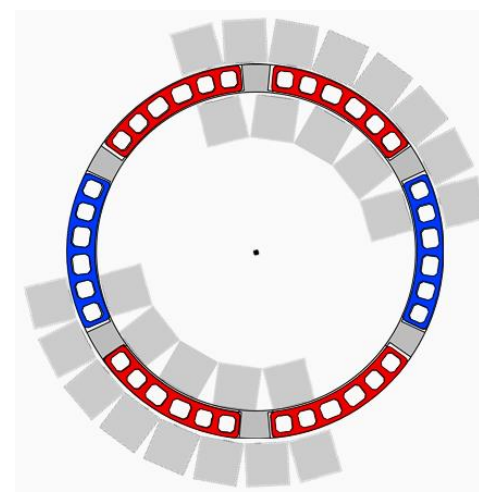

(c)

The torque developed by the rotor in the movement was then used to comparatively analyze the performance of these different configurations of control. This analysis was done using the 3D magnetostatic analysis approach that was validated experimentally in the previous section; additionally, the simulation of the permanent magnets assembly of the motor was composed with the magnetic material plates. Using the experimentally validated model of the permanent magnets assembly presented in section 5.2. As well as the adequate magnetic properties of the $\mathrm{Gd}_{4.7} \mathrm{Nd}_{0.3} \mathrm{Si}_{4}$ compound, that showed to reproduce in the simulation the results of the measured magnetic force (Figure 72.b).

The models used in the simulations are shown in Figure 75. In the figure, the blue plates represent the magnetic material at the temperature of $298 \mathrm{~K}$, while the red plates represent the material at the temperature of $343 \mathrm{~K}$. For these simulations, the permanent magnets assembly remains static, while the plates are rotated in the counter-clockwise direction, from the starting point shown in the image, to a position $120^{\circ}$ in the direction of rotation, with steps of $1^{\circ}$. The magnetically induced torque on the plates will then represent the torque acting to rotate the permanent magnets during the operation of the motor. The three poles configuration of control (Figure 74.c) was not directly simulated since this configuration represents the composition of two mirrored models of the configuration with six plates, this way the torque for of this design was obtained by doubling that produced by the model simulated using Figure 75.b. 
Figure 75: Models of the permanent magnets assembly with the magnetic material plates, used to compare a Rotary TMM motor with control configuration of three plates (a), and with six plates (b).

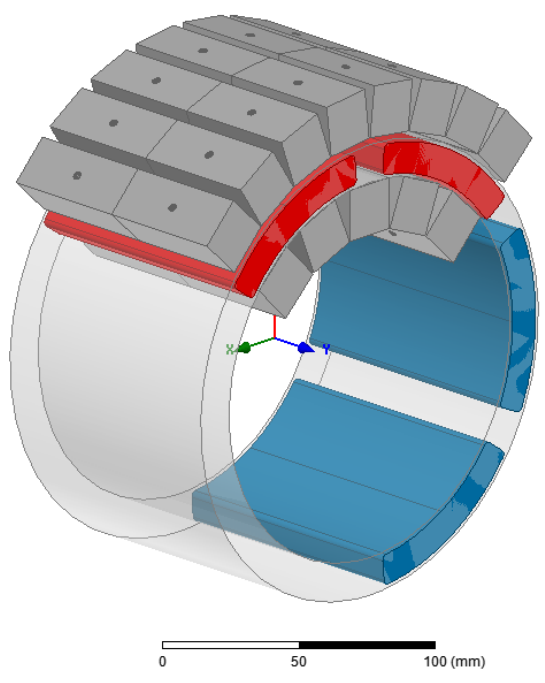

(a)

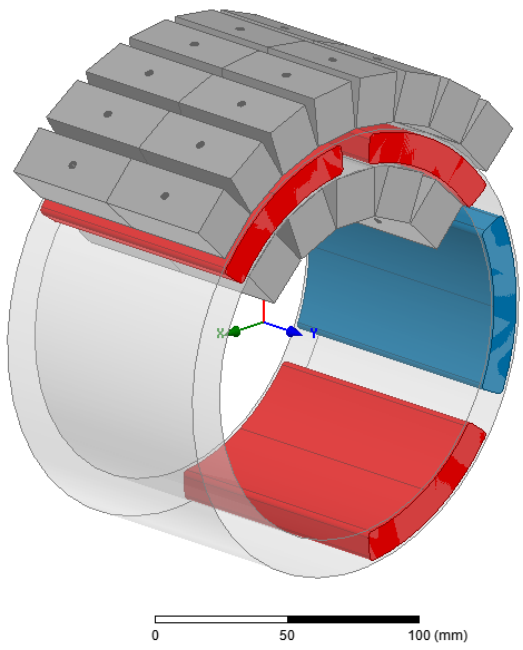

(b)

The simulations were done using the energy error as the convergence control parameter, with convergence assumed with error inferior to $0.05 \%$. The meshing control of maximum length was applied, to a maximum of $2 \mathrm{~mm}$ for the solid elements and the "dummy" region, and $10 \mathrm{~mm}$ for the surrounding atmosphere. The resulting mesh after convergence had a total of $2.84 \cdot 10^{6}$ elements.

From these simulations, the torque produced by the rotor of the motor was obtained for the three different configurations of control proposed, as presented in Figure 76. The simulations indicate that there is a small difference between the torques obtained using the designs simulated with the models shown in Figure 75, although, it is important to notice that in the six poles configuration the (Figure 74.b) the movement starts from the simulated angle of $30^{\circ}$ ( $\theta_{0}$ in Figure $74 . \mathrm{b}$ ), with the rotation of the plates up to the position of $90^{\circ}$. Hence, the new design, with six plates acted independently, presents a higher starting torque, with the movement occurring only in higher intensity regions of the torque. Lastly, the design with two opposing plates of the rotor active (Figure 74.c), presents the highest peak torque, since it can be described as the composition of two times the six poles configuration.

${ }^{2}$ The "dummy" region is defined by the annular region where the movement occurs, indicated by the shaded are in Figure 75. It is modeled as a separate solid to be used in the meshing refinement process. 
Figure 76: The torque produced by the three different configurations of control studied, obtained through the 3D magnetostatic simulation.

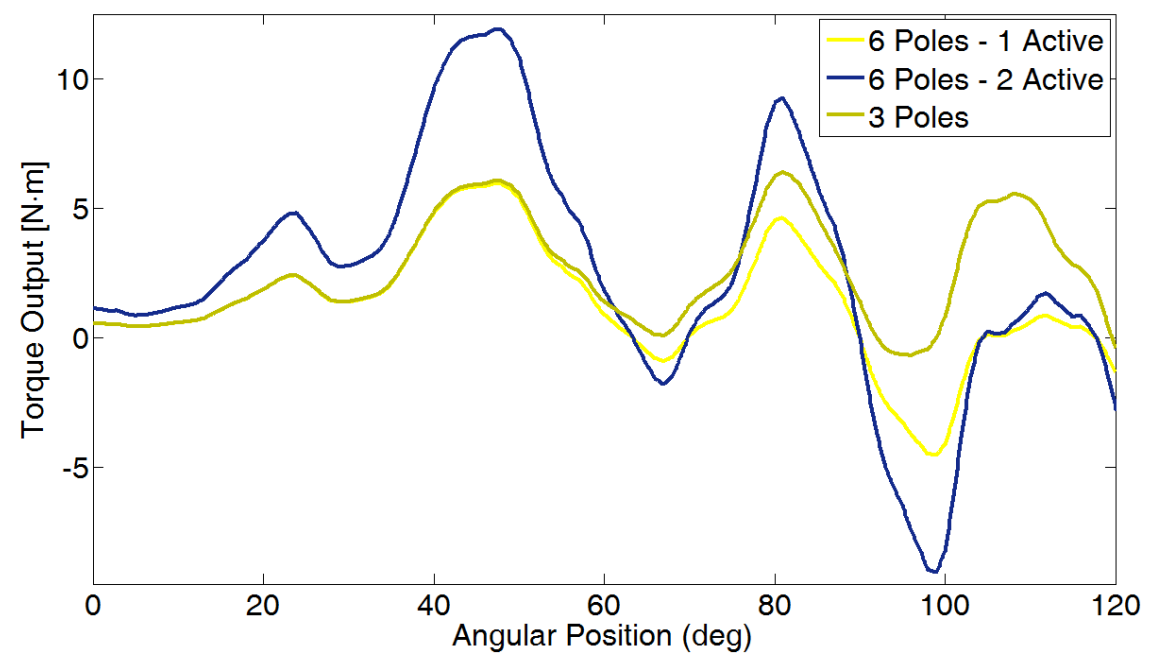

It is important to notice that the torque values obtained from the simulations are expected to present a valid approximation of the torque developed in the motor. In the first place because the applied magnetic field strength produced by the permanent magnets assembly in the simulation was validated experimentally, as shown in section 5.2. Also, the magnetic properties of the $\mathrm{Gd}_{4.7} \mathrm{Nd}_{0.3} \mathrm{Si}_{5}$ compound were measured, and the data applied in the force simulations showed a good approximation to the simulations, presented in section 7.2. Lastly, the modeling of the permanent magnets and the magnetic material plates followed that of the system actually built, with the plates shown in Figure 75, having the same internal channels as those fabricated for the motor, and the same volume of magnetic material as the plates used in the validated simulations of heat transfer shown in 6.1.

Despite presenting a valid approximation of the magnetic torque produced in the Rotary TMM, this approximation will not necessarily indicate the available torque from the motor, or can be directly used to determine its work output. As the Rotary TMM works developing impulsive rotary steps, part of the work produced will be expended by the rotor dynamics. Also, the magnetostatic simulation does not take into account the Eddy currents that are induced by the movement of the applied magnetic field on the magnetic material plates and on the support structure of the stator, which generates a magnetic damping effect on the rotor, also reducing the available work output.

This way, the integrated approach between experimental analysis and simulation was used to analyze the available power output of the rotor. The experiments were conducted 
using the complete Rotary motor construction, to which a rotary encoder, the Autonics encoder model E50S8-1000-3-T-24 already applied in the experiments to measure the applied magnetic field as presented in section 5.2, was applied to determine the angular position of the rotor. A magnetic break was used to electronically control the holding of the rotor into the initial position, as indicated by the red arrow in Figure 77.

Figure 77: The built Rotary TMM, with the data acquisition system and the magnetic break used to determine the peak power output of the rotor.

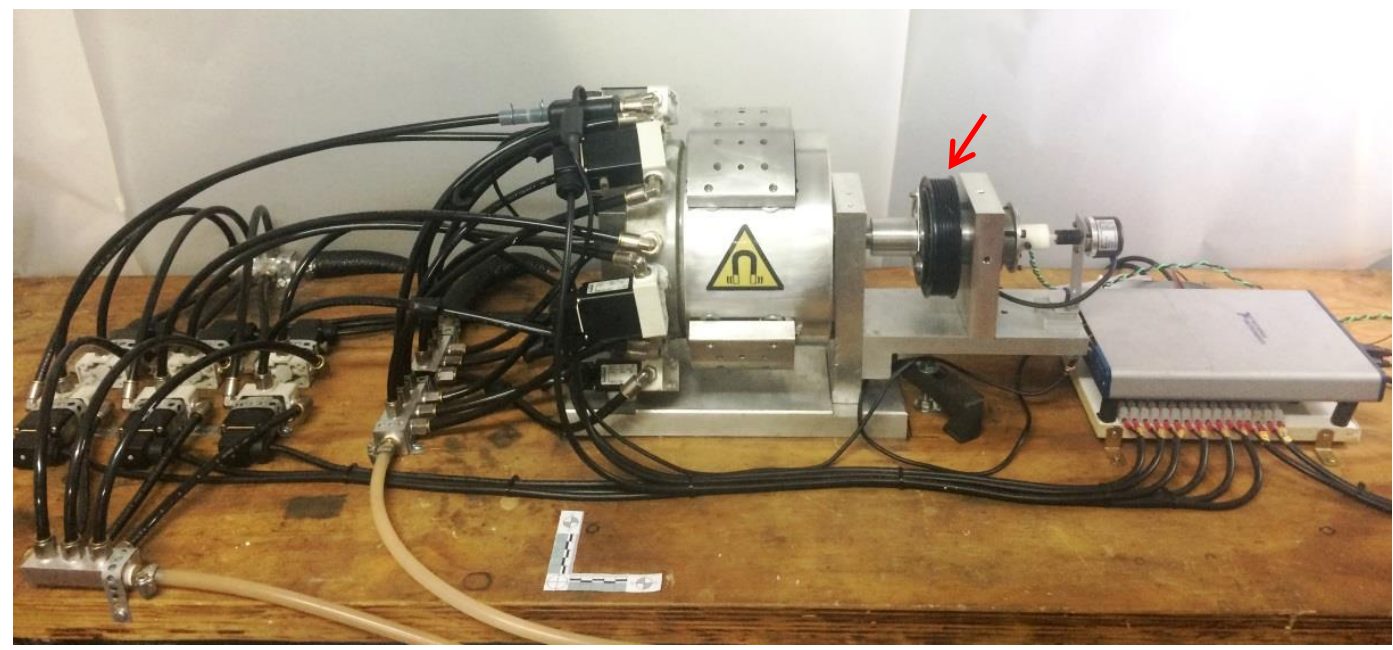

In the experiment, the rotor was held into an initial position, while the plates were heated and cooled in the configuration shown in Figure 74.c, until thermal equilibrium with the heat source and sink, at which point the magnetic brake was turned off and the speed of rotation registered by the rotary encoder. The observed movement of the rotor occurs in a fast pulse, with the rotor oscillating until it stabilizes at the next position, where the magnetic brake was turned on again, and the experiment restarted. The data acquisition was made using the NI-USB-6363 DAQ, with an automatic experimental procedure developed through the LabVIEW software. As the step rotation developed by the motor is fast, a data acquisition rate of $4 \mathrm{kHz}$ was used in these measurements.

The measured data could then be compared to the dynamic simulation of the rotor, while it is moved by the magnetic torque obtained through the magnetostatic simulation. The power output of the rotor is then obtained by combining these two simulation processes. The dynamic simulation was performed using the model of the rotor in the software Inventor, shown in Figure 78. This model used the density of the built materials to reproduce the same 
masses as the constructed rotor parts. The total mass of the rotor in the simulation was of $20.81 \mathrm{~kg}$, which was also verified experimentally. From which the software calculated the moment of inertia of the rotating axis to be $144.57 \mathrm{~g} \cdot \mathrm{m}^{2}$

Figure 78: The model of the rotor of the rotary thermomagnetic motor, used in the dynamic analyses.

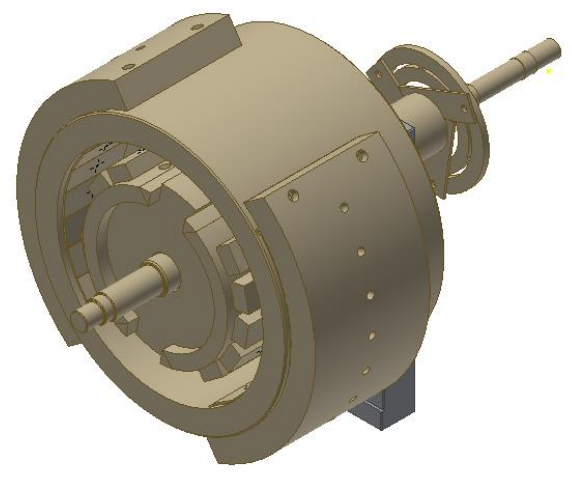

The first results of the dynamic simulation showed a fast angular acceleration, with the motor then continually rotating. This behavior indicated that the free rotation of the axis was not an actual representation of the physics involved, mainly because the magnetic damping was not considered in this first simulation, by then adjusting the damping factor to a value of $7.2 \mathrm{~N} \cdot \mathrm{mm} \cdot \mathrm{s} / \mathrm{deg}$, the movement of the axis developed the same oscillation and stabilization as the movement of the rotor in the experiments. The comparison between the simulation results and the data obtained experimentally is shown in Figure 79.

Figure 79: Comparison between the measured variation of the angular position of the rotor, during a single step, from the built system and from the dynamic simulation.

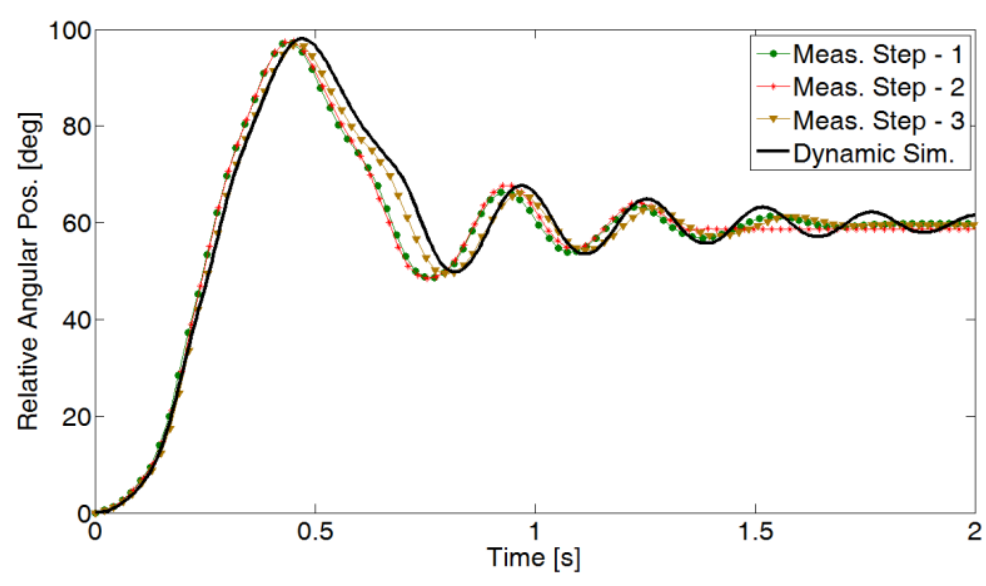

The high data acquisition rate used in the experiment and the good resolution of the rotary encoder was capable of adequately describe the angular position of the rotor with 
respect to time during the rotation. With that the angular position of the rotor $(\omega)$ can then be used to determine its angular velocity, deriving the position in respect to time, and this value, calculated from the experimental data, can be compared to the angular velocity describe by the rotor in the simulation, as presented in Figure 80.a. As can be seen, the discontinuities in the measured data, although small, produce a noisy angular velocity result, while the simulations produced a smooth behavior that fit the data from the measurements. Then using the simulations, the angular acceleration $(\alpha)$ of the rotor is determined, as shown in Figure 80.b.

Figure 80: In (a) the angular velocity developed by the Rotary TMM, comparing data from the simulation and measurements, and in (b) the simulated angular acceleration of the rotor.

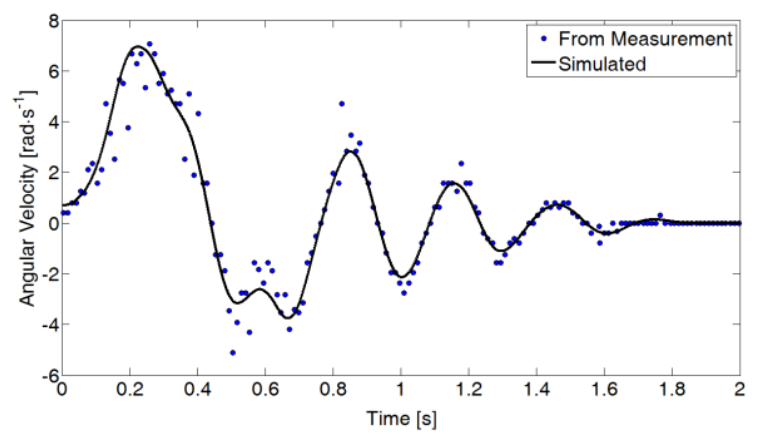

(a)

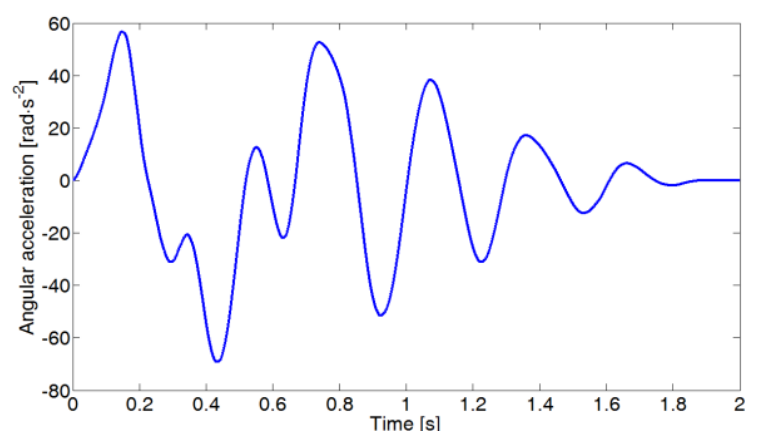

(b)

Using these data, the torque output of the rotor and the power produced can be calculated with:

$$
\begin{gathered}
T_{\text {out }}=I \cdot \alpha \\
P_{\text {mot }}=T_{\text {out }} \cdot \omega
\end{gathered}
$$

Where $I$ is the moment of inertia of the rotor. From the simulations the torque output actually developed by the rotor is characterized to have a peak of $8.2 \mathrm{~N} \cdot \mathrm{m}$, which represents $69 \%$ of the maximum torque produced by the magnets, of $11.89 \mathrm{~N} \cdot \mathrm{m}$, as obtained from the magnetostatic simulations, this loss represents the influence of the dynamic properties of the rotor and the magnetic damping caused by the fast movement of the permanent magnets assembly. The power output from the axis presented a peak of $50.88 \mathrm{~W}$, with a high oscillatory characteristic.

The built thermomagnetic motor can then be characterized by developing the control system that executes the configuration presented in Figure 74.c, which presented the most 
promising torque and power outputs. The control system developed uses the angular position acquired with the use of the angular encoder already built in the motor (Figure 77), acting to change the position of the solenoid valves properly, and with that keep a continuous rotation in the system. A cycle of the built motor is then presented in Figure 81.a, in which every continuous section indicates the period of heat transfer of the motor, the period of one rotation of the motor was of $54.43 \pm 2.07 \mathrm{~s}$, with heat transfer periods of $9.33 \pm 1.09 \mathrm{~s}$. A single step of the motor in operation is presented in Figure 81.b.

Figure 81: The cycle developed by the Rotary TMM built, in (a) a complete rotation of the motor, and in (b) the oscillation in the position during a single step.

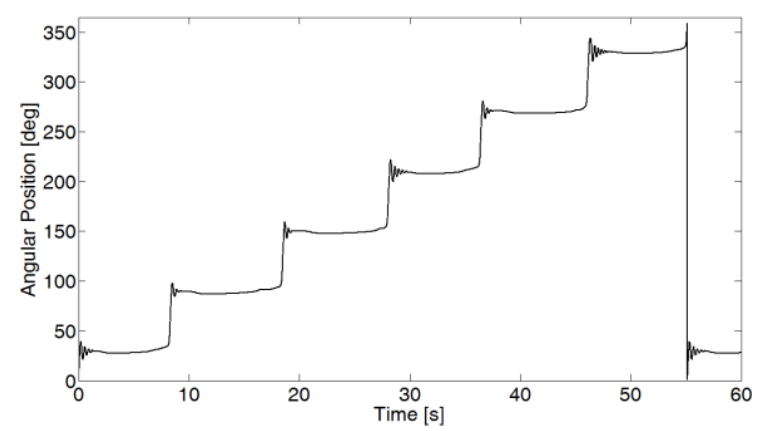

(a)

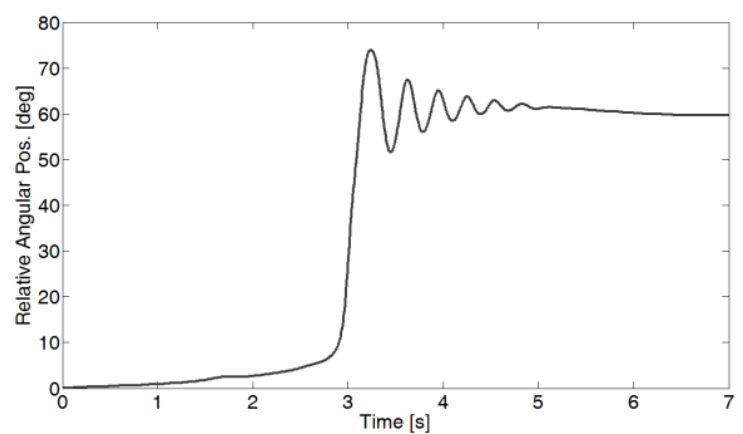

(b)

During the operation, the Rotary TMM develops a lower power output than the maximum output obtainable from the temperature differences. As explored in section 7.1, given constant heat transfer rates, the period of operation of the motor will increase to achieve the demanded power output. This characteristic also allows the motor to operate within a range of heat source temperatures, with the compromise that smaller temperature differences will produce smaller power outputs with a larger operating time. 


\section{IMPROVEMENTS IN THE BUILT ROTARY THERMOMAGNETIC MOTOR}

Throughout this thesis, the performance and working characteristics developed by two designs of Tesla type thermomagnetic motors were studied using both numerical simulations and experimental analyses. This integrated approach allowed the validation of the computational models describing individually the three central physical phenomena occurring in these motors, the interaction of a magnetic material with an applied magnetic field, the transfer of heat to the magnetic material, and the dynamics involved in the work and power produced. Based on the experimentally validated models and simulation approaches used, new constructive characteristics for the motor developed can be proposed, aiming to improve its performance and functionality.

One important point to consider is that these changes in the computational models must not violate the physical constraints that exist in the actual realization of the motors. Since the objective of the proposed alterations to the models is to obtain guidelines and parameters that may help to design and build new motors with improved performance. For instance, the heat transfer rate to the plate modeled in the CFD simulations, presented in section 6.1, could be significantly increased by applying very high flow rates of water; however these simulations could not be validated with the CFD model that reproduced the heat transfer measured in the presently built motor (Figure 64), as higher flowing speeds would represent significant changes in the parameters of turbulence and on the interaction between the magnetic material and the water. Furthermore, a limit on the maximum flow rate developed in the motor is also imposed by the mechanical stability of the magnetic material plates, which could not, as they stand now, support the high pressures involved.

The first improvement to be evaluated for the Rotary TMM proposed will be to change the magnetic material used. Although the magnetic material used in the TMM motor built was capable of producing a measurable power output, this output could be significantly improved by the adoption of other magnetic materials. From the measured magnetization properties of the $\mathrm{Gd}_{4.7} \mathrm{Nd}_{0.3} \mathrm{Si}_{5}$ compound presented in Figure 36, it becomes clear that its 
moment magnetization is at least four times lower than that of Gd, shown in Figure 22, for the same applied magnetic field with both materials at a temperature below their respective critical temperatures $\left(T_{C}\right)$. However, Gadolinium does not present a viable alternative for the application proposed of energy conversions with heat sources in the range of 343 to $353 \mathrm{~K}$, due to its $T_{C}$ of $293 \mathrm{~K}$, being below room temperature. A few alternative materials that are presented in the literature, and could in principle be used in the Rotary TMM developed, include: $\mathrm{Ge}_{5} \mathrm{Si}_{4}, \mathrm{MnFeP}, \mathrm{LaFeSi}, \mathrm{Fe}_{17} \mathrm{Nd}_{2}$, and MnAs.

An interesting characteristic to be explored in these alternative materials, is to use magnetic materials that present a "first-order" magnetic transition. "First-order" materials have a transition in which the magnetization changes discontinuously at $T_{C}$, with a latent heat associated with the phase transition, and in most cases presenting a high thermal hysteresis in the thermomagnetic transition, meaning that the critical transition temperature will be different for the heating and cooling processes. While the thermal hysteresis is usually associated with a loss of energy, especially for the applications in magnetocaloric refrigeration, and therefore attempts have been made to reduce its magnitude (LEE et al., 2018), for thermomagnetic motors this effect could, in fact, represent an increase in the efficiency (BESSA et al., 2017).

To study this possibility the test stand, developed to characterize the behavior of an idealized thermomagnetic motor, section 4.1, was applied, using a sample of MnAs. With that, obtaining a curve of the magnetization work of the MnAs, as a function of its temperature and applied magnetic field change, similarly to the one shown in Figure 70 for Gadolinium. The MnAs presents a first-order thermomagnetic transition, and critical temperature $\left(T_{C}\right)$ while heating of $318 \mathrm{~K}$, and also a thermal hysteresis of $10 \mathrm{~K}$, hence its $T_{C}$ is of $308 \mathrm{~K}$ while the material is being cooled. The results obtaining for the heating and cooling of the material are shown in Figure 82. 
Figure 82: The magnetization work of the MnAs, as a function of its temperature and applied magnetic field change, for the heating (a) and cooling (b) processes.

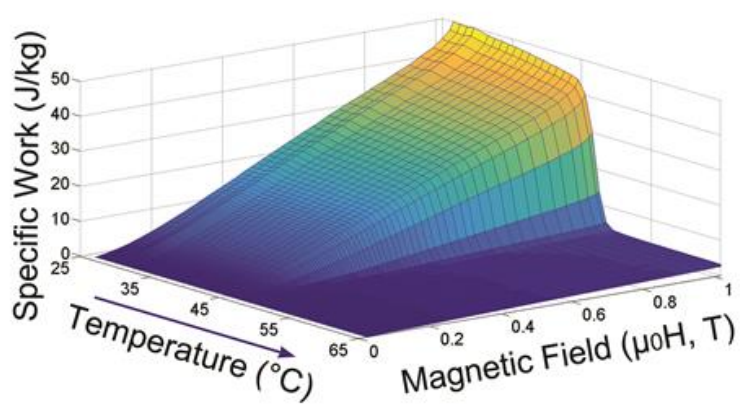

(a)

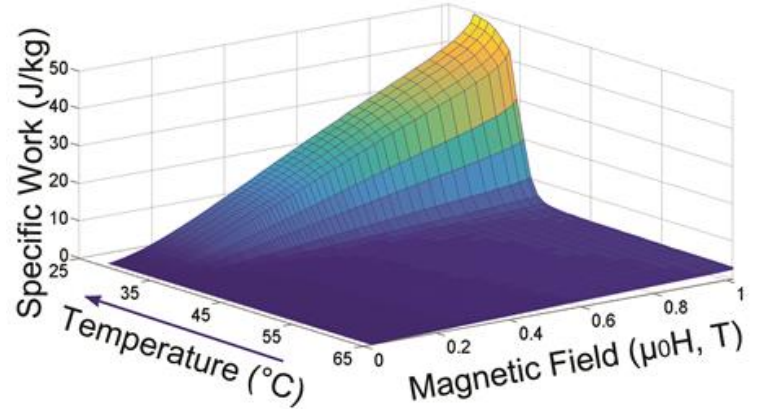

(b)

The analysis of the behavior of an idealized thermomagnetic motor from the curves shown in Figure 82 can be done by considering that, the material is in a high magnetic field during the heating (a) process, with an external mechanical work being applied to remove the material from the magnetic field when it reaches a temperature higher than $T_{C}$. Then the material is cooled (b) in a low applied magnetic field, until it reaches a temperature below $T_{C}$, at which point the material is attracted to the high magnetic field region, producing mechanical work from the magnetic force induced in the material.

From this exploration, it can be concluded that the application of the MnAs, in the idealized TMM proposed, is capable of producing as much work as the use of $\mathrm{Gd}$, for the same mass of material. While the $T_{C}$ of the MnAs, of $318 \mathrm{~K}$ also makes it a good candidate for the application in energy conversion for which the Rotary TMM was designed. Another advantage that can be introduced by the first-order transition observed in this material is the sharp thermomagnetic transition, in opposition to the observed for the Gadolinium in Figure 70. This sharp transition would cause the thermomagnetic motor to produce its maximum power output with a small temperature difference, even taking into account the thermal hysteresis.

Another improvement evaluated for the Rotary TMM developed is to change the permanent magnets assembly used in the motor. Since the torque produced by the motor is dependent on the gradient of the applied magnetic field, higher field strengths could be used to produce a motor with increased load capacity. These amplified magnetic fields could be provided by electromagnets or superconducting electromagnets, although, as explored in chapter 5, the use of such systems could represent a reduction of the efficiency of the motors 
and a significant increase in complexity. More realistically the use of different permanent magnets assemblies is proposed.

The permanent magnets assemblies used in the Rotary TMM developed are based on a design similar to that initially proposed by Halbach (1980). Although this construction did promote an adequate field concentration inside the desired region, this design could be improved by the combination of permanent magnets and soft ferromagnetic materials. This approach was used by Bjørk et al. (2010), in a permanent magnets assembly that was able to produce a maximum applied magnetic field strength of $1.24 \mathrm{~T}$, with an average flux density of $0.9 \mathrm{~T}$, inside the desired field region with a volume of $2 \cdot 10^{-3} \mathrm{~m}^{3}$. This would represent doubling the maximum applied magnetic field strength obtained in the current system. Another approach would be to redesign the permanent magnets assembly using a topology optimization approach, which has been shown to be capable of concentrating a homogeneous magnetic field to increase the amplitude of the applied magnetic field in up to $111 \%$ (BJØRK; BAHL; INSINGA, 2017). With the increased cost of having permanent magnets with unusual magnetization directions and shapes, which would have to custom made.

The improvement of the heat transfer between the magnetic materials in the motor and the water flow presents a challenging problem to be evaluated through the developed and validated model. As previously mentioned any alteration in this system would have to respect the physical constraints of the built motor to produce valid results. One alteration explored was that of redesigning the connection of fluid between the magnetic material plates and the support, which initially presented a tight tolerance for the assembly. A change was tested using the CFD simulation by making the connectors larger and improving the alignment between the inlets of the magnetic material plate and the support. The results showed that the pressure drop was reduced by $20 \%$ for the same mass flow rate, and also that the heat transfer rate was increased.

A more considerable increase in the heat transfer speeds could be obtained by changing the magnetic material plates. In first place the $\mathrm{Gd}_{4.7} \mathrm{Nd}_{0.3} \mathrm{Si}_{4}$ compound presented a low thermal conductivity $(k)$, as shown in Figure 98. This can be partially attributed to the presence of porosity in the compacted plates, and also to the Epoxy agglomerating material, which does not provide excellent heat transfer. A second approach would be to modify the 
internal channels on the plate, it has been demonstrated that the heat transfer can be substantially increased by increasing the contact area between the magnetic material and the fluid on the channels, which can be done without necessarily decreasing the volume of magnetic material, but rather by changing the shape of the channels and adopting the use of a larger number of smaller channels (FERREIRA et al., 2014)

The dynamics of the rotor of the built Rotary TMM also present a chance for improvement of these systems. In the first place, the mass of the magnets and its supports in the rotor introduce a substantial loss of mechanical power, since part of the magnetic torque output is expended in accelerating the rotor. An alternative analyzed, using the dynamic simulation approach shown in section 7.3, was to change the material of the magnets support parts, which are initially made of aluminum, adopting carbon fiber reinforced polymer (CFRP) as a substitute material. With that the mass of the rotor was reduced to $14.8 \mathrm{~kg}$, a reduction of $30 \%$ from the original mass, and also the moment of inertia of the rotor was reduced to $101.6 \mathrm{~g} \cdot \mathrm{m}^{2}$.

Another aspect of the dynamics of the rotor that can be optimized is the reduction of the magnetically induced damping of the rotor, which is caused by the rapid movement of the magnetic field over the stator of the Rotary TMM, and the consequent induction of Eddy currents on the magnetic material plates the support. Though the effect is small on the magnetic material plates, that due to the agglomeration process present a low electrical conductivity, it is substantial on the support of the plates, currently made of aluminum. By changing the support to one made of non-magnetic stainless steel, material which presents an electrical conductivity of $5 \%$ that of aluminum, the magnetic damping can be reduced to at least one order of magnitude; another alternative would be the use of a rigid polymer.

The third aspect of the dynamics of the rotor that was analyzed was the oscillatory character of the movement, as can be seen in Figure 79 and Figure 81.b. This oscillation is caused by the inertia of the rotary movement, and also by the way the magnetic torque is produced in the rotor, Figure 76. These oscillations are reduced with the application of a load in the rotor, though, due to the increasing characteristic of the magnetic torque in the rotor, higher loads may cause the rotor to lose the starting torque and cause blockage of the rotor. 
In the patent originally proposed by Coelho, Gama, and Silva (2012), a flywheel and ratchet mechanism is proposed as a way to convert the pulsating rotation of the motor into a continuous rotation; this flywheel would also present an alternative to reduce the oscillations in the rotor. By using the dynamics simulation the torque output of the rotor, analyzed in section 7.3, is obtained, as presented in Figure 83. In it, the positive torque output presents that obtained in the direction of rotation of the motor, indicated within red in Figure 83. This positive output would represent the available torque for a flywheel, while the ratchet mechanism would absorb the negative torque.

Figure 83: Torque produced by the rotor of the Rotary TMM, calculated using the dynamic simulation data.

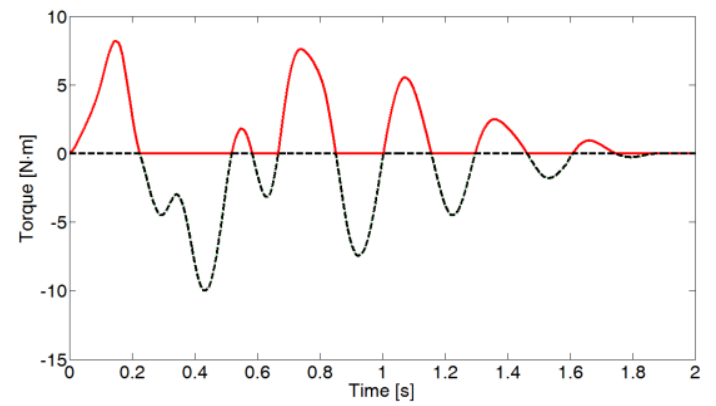

Finally, some improvements could be applied to the control system of the Rotary TMM developed. These present a more easily implemented alternative for the use in the already built system. Since the control system is based on the NI-USB-6363 DAQ, the control logic developed through the LabVIEW software, these improvements can be employed without changes in the built motor.

The first alternative proposed was a change in the activation control of the magnetic plates, using logic similar to that proposed in the microstepping of electric stepper motors, based on the control sequence shown in Figure 74.c. In this new logic, the control on the hydraulic valves is changed with bases on two factors, the position of the rotor and the temperature developed by the plates being cooled. After the movement of the rotor, only two opposing valves receive the flow of cold water, in the same manner as presented in Figure 74.c. Then, as these plates are partially cooled, the plates that would constitute the next step in the control start to also receive cold water, with the motor presenting the state shown in Figure 84 , with the plates heat presented in red, the plates that were initially activated in blue and the plates that constitute the next step in orange. 
Figure 84: Change in the control design for the operation of the motor with two cold plates in the six poles configuration, adding the 'half-step' logic to increase the rotational speed.

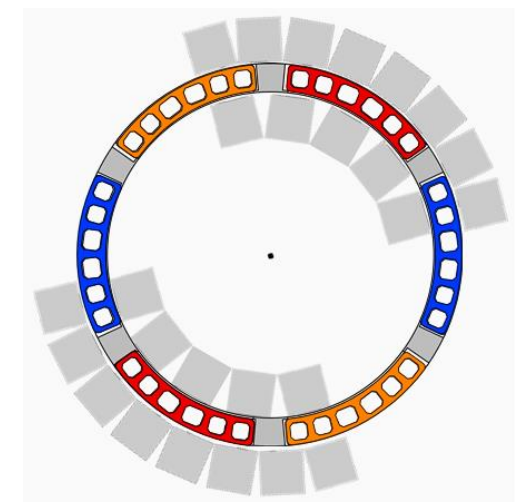

This alternative control of the valves would be capable of developing higher rotational frequencies of the motor, since the proactive cooling of the plates reduces the period of heat transfer in the next step of rotation. An expected drawback from this approach is the reduction of the torque output of the rotor, since the plates being proactively cooled will present an opposing torque to the direction of rotation of the motor.

However, this technique did not produce satisfactory results in the built motor, for one part the temperature of the plates, measured using $\mathrm{N}$-type thermocouples, did not present a stable signal to be used in the control logic developed. Additionally, because the critical temperature $\left(\mathrm{T}_{\mathrm{C}}\right)$ of the material, being closer to the heat source temperature than to the heat sink, ended up making the cooling times faster than the heating times, this was also observed in the heat transfer simulations, as can be seen in Figure 65. Nonetheless, this approach still presents a valid alternative to optimize the power output of the Rotary TMM, if different heat transfer rates are applied to the cooling and heating cycles, e.g., by adopting different mass flow rates for the hot and the cold streams, or if a magnetic material with different $\mathrm{T}_{\mathrm{C}}$ is to be used.

Another alternative to improve the performance of the Rotary TMM, through changes in the control system, is to change the way in which the inlet and outlet valves of the plate are controlled. From the thermal cycling of a plate of magnetic material, as shown in Figure 64, the amount of heat being received by the plate was determined to be the difference between the heat flow in the inlet and the outlet, as calculated using equation 6.3. However, during the operation of the motor, as one plate is being heated, the next is being cooled simultaneously; 
this can be visualized in Figure 85.a, for the heating cycle simulated. This way, by controlling the state of the outlet valves independently from the position of the inlets, the water flow can be redirected to the reservoir for which the temperature of the outlet is the closest. In Figure 85.a the color of the lines indicate whether the valves are connected to the hot water reservoir (red) or the cold reservoir (blue), with the moments in which the outlet valves are actuated shown by the dotted lines.

With the change in the control of the outlet valves, the temperature of the water flowing from and back into the hot reservoir is determined by Figure 85.b, from which, using equation 6.3, the heating flow of the motor as a whole can be calculated. Resulting in a total of $156.87 \mathrm{~kJ}$ per cycle, which represents a reduction of $63 \%$ of the $424.6 \mathrm{~kJ}$, obtained the heating cycle with inlet and outlet actuated simultaneously. In essence, this change in the control promotes the regeneration of the heat from the plate being cooled, which reduces the amount of energy expended in the motor while keeping the same amount of work produced, with that substantially increasing the efficiency of the system.

Figure 85: An alternative control to recover the heat from the outflow of the plates of magnetic material in the Rotary TMM, in (a) the thermal cycling individually developed by the two parallel plates of the TMM, in (b) the equivalent heating cycle with the recovery of heat from the plate being cooled.

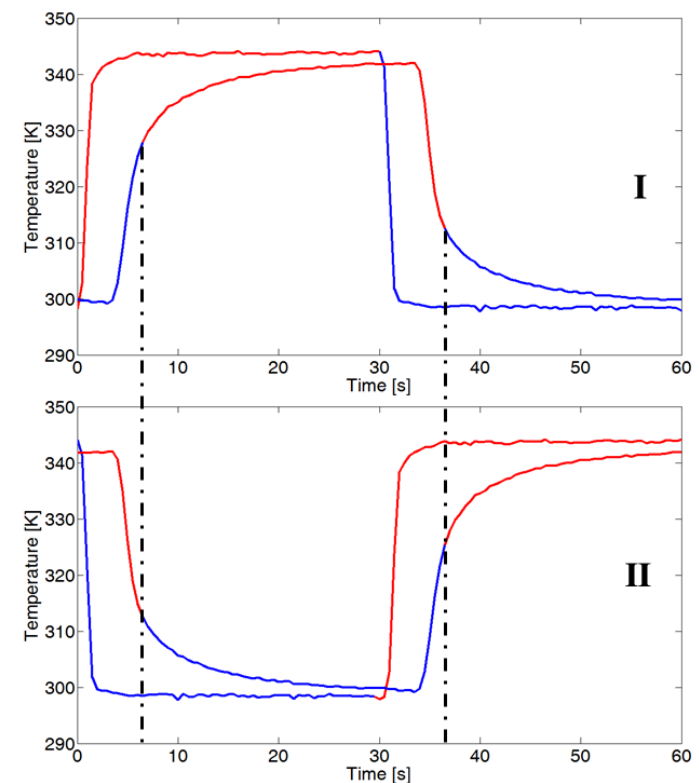

(a)

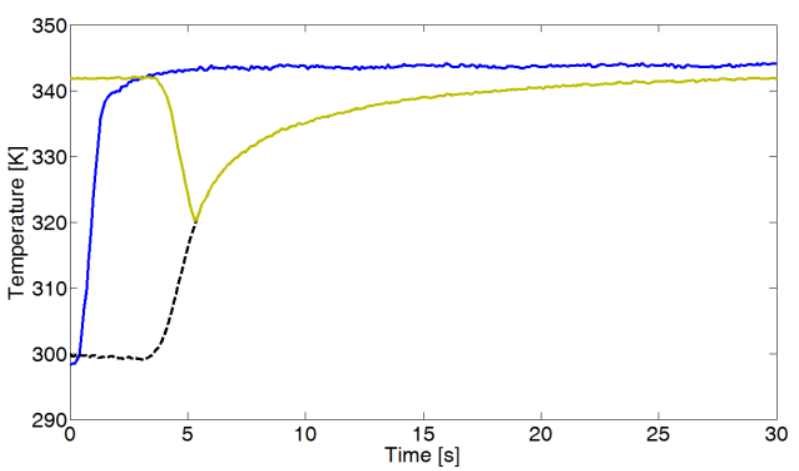

(b)

This regeneration technique can be easily implemented in the control system, both by using the temperature of the outlet as a control variable, or by adopting a constant delay 
between the change in the inlet valves, which is determined by the position of the rotor and the design executed, and the activation of the outlet valves. The increase in complexity caused by the necessity of having the inlet and outlet valves controlled independently is compensated by the considerable increase in the efficiency of the motor. 


\section{CONCLUSIONS}

This thesis presents the development of a Rotary thermomagnetic motor, through experimentally validated simulations of its systems, and analysis of a laboratory scale demonstrator. The motor, which operated with a heat source between 343 and $353 \mathrm{~K}$, was shown to produce a measurable power output, though the demonstrator built presented many aspects to be improved. Some improvements to the system were then evaluated using the simulation tools, showing that there is an untapped potential of application of this technology in the conversion of low-grade heat.

The literature review revealed that exist two distinct approaches to the energy conversion with thermomagnetic devices: thermomagnetic generators, that propose the direct conversion from heat into electricity; and thermomagnetic motors, that promote the conversion from heat into mechanical work. The motors can then be subdivided into two categories: the "Edison type" and the "Tesla type" of thermomagnetic motor. The thermodynamic analysis of the two types of thermomagnetic motors showed that given the same heat sources temperature and applied magnetic field strength, the "Tesla type" of thermomagnetic devices will present a larger work output and efficiency.

There have been some attempts to apply these motors on a laboratory scale, the

evolution of these previously developed designs were analyzed using the "Technology Readiness Level" tool. From which it was concluded that most of the research published on this subject is not on the technology development level, but instead presented as a proof of concept and in the basic research level. The developed Rotary TMM uses the "Tesla type" of design, which presents as an innovative approach to the application of thermomagnetic motors in the proposed range of temperatures and expected mechanical power output.

The analysis of the thermomagnetic motor was done by dividing it into the three main physical phenomena it encompasses: the magnetic field source, the heat transfer processes involved in the change of temperature of the magnetic material, and the system dynamics and power production. Each of these systems was modeled using computational tools. These 
models were then validated according to the data measured, obtained from a test stand of an idealized thermomagnetic motor, and for the built Rotary TMM.

The first part was the study of the magnetic field sources used in the motor. This was initially done using a magnetostatic simulation method, for both the test stand and the permanent magnets assembly proposed for the built motor. Using the applied magnetic field strength as a validation parameter, the data obtained in the simulation and determined experimentally were compared, resulting in the validation of the simulation method, as long as, the model adopts an adequate representation of the concessions done in the making of the permanent magnets assembly.

The following step was to study the way in which heat is transferred to the plate of magnetic materials of the motor. As these plates are being cyclically heated, this process occurs entirely in a transient manner, with the continuous alternation between the flow of hot and cold water. This characteristic adds another element of complexity to the CFD analysis used, which is also composed by the difficulties in characterizing the fluid flow and determining the heat transfer properties of the uncommon $\mathrm{Gd}_{4.7} \mathrm{Nd}_{0.3} \mathrm{Si}_{4}$ compound used in the plates. The properties of the magnetic material were measured using the specific heat measurement apparatus built by Monteiro et al. (2014), and a thermal conductivity measurement apparatus built in the laboratory (Appendix 2). Despite these difficulties, the CFD analysis was capable of adequately reproduce the fluid flow and heat transfer characteristics measured in the built motor.

The last physical system studied was the dynamics of the thermomagnetic motor. This step comprises the characterization of the magnetic forces obtained by the interaction between the permanent magnets and the material, that was measured using the test stand and simulated, from which the magnetostatic simulation was validated. Also, the mechanical work that is produced as the material is moved inside the magnetic field was determined, defining the characteristics of an idealized TMM. Lastly, the dynamic behavior of the rotor in the built motor was studied, considering the inertia in the system and the losses induced by the moving magnetic field. This analysis involved the combination of the torque calculated using magnetostatic simulations and a multibody dynamics simulation. The simulated results were then compared to the measured data from the rotor of the built thermomagnetic motor. 
Concluding that, the dynamics of the built motor were reproduced using the simulation methods.

Finally, the validated simulated systems of the motor are used to propose and analyze improvements to the motor built, aiming to optimize its performance and efficiency. The importance of these analyses is not only to better the design built but also to instruct the project of new thermomagnetic motors working with the same principles. One conclusion from these changes in the motor is that a tradeoff between performance and complexity of the motors must be observed, since some improvements would make the motors excessively complex and with that more expensive. This also demonstrates the importance of using the simulations for the analysis of the improvements proposed, since a cost analysis of these alternatives can be run, determining the most effective ways to optimize the motors.

The built Rotary TMM technology demonstrator constitutes a substantial advancement on the use of the thermomagnetic motors, operating with a "Tesla type" of design for the conversion of energy from low-grade heat sources. The demonstrator provides a laboratory scale validation of the technology, with computational and experimental proof of concept characterization, however without yet promoting the integration between the built motor and the system that will eventually make use of the mechanical power produced. The motor built is also largely based on systems that are calibrated or constructed specifically it, and the availability of these systems in a commercial context has not been explored. With that, the technology readiness assessment analysis of the work developed in this thesis puts it into a TRL 3 scale, with the advancements made in the simulation approaching a TRL 4 level, and therefore in the frontier between the proof of feasibility and the technological development levels.

\subsection{Recommendations for future work}

One of the main aspects that need to be advanced is in the research of the magnetic materials used in the operation of the motor. The material used in the motor built. the $\mathrm{Gd}_{4.7} \mathrm{Nd}_{0.3} \mathrm{Si}_{4}$ compound had to be fabricated from its composing elements and prepared into the shapes of the magnetic plates using a technique of agglomeration under high pressure (Appendix 1). The lack of commercially available materials presents a significant challenge 
for the advancement of the technology of thermomagnetic motors. Another important difficulty is given by the lack of proper characterization of the physical properties of the materials that have been reported in the literature, while many reports can be found of magnetic materials with critical temperature $\left(\mathrm{T}_{\mathrm{C}}\right)$ in the desired range, most of the reports fail to explore the thermal and mechanical properties that would allow their evaluation for the application on the motors.

To continue the research developed in this thesis, the improvements proposed for the built prototype should be applied to the design of a new Rotary TMM. With the possibility of increasing the torque produced by the motor, using different permanent magnets arrangements, reducing the dynamic losses of the rotor, and a magnetic material with better magnetic properties. At the same time increasing the power output could be increased, by optimizing the design of the plates of magnetic materials in order to increase the heat transfer rate between the plates and the water, and with that reducing the period of rotation of the motor. Lastly, the efficiency of the system could be improved by the use of magnetic materials with first-order transition, and also by using regenerating the heat from the magnetic plates through the use of better valve control strategies.

For the future application of the technology of thermomagnetic motors, the points of optimization presented in this thesis could be used as general guidelines. The application of these improvements should also be followed by a cost evaluation. A general cost evaluation of the TMM is also an important point to be developed since other technologies have been proposed for the application of energy conversion from low-grade heat sources. Another point to take into account is to analyze the environmental impact of these motors, using a life-cycle analysis and encompassing not only the fact that the motors can be used to the conversion of "clean energy" sources, but also the impact of production of the magnets and magnetic materials, and how these materials could be eventually recycled.

Though this thesis is focused on evaluating the possibilities of applying the TMM for the energy conversion from low-grade heat sources, these motors are not limited to be applied in this range of temperatures. By changing the magnetic materials used for materials with a higher $\mathrm{T}_{\mathrm{C}}$, the motors could be operated with sources at different temperatures, with that, these motors could compete with more traditional vapor expansion turbines, while 
possibly not competitive from an economic perspective, the safety in the energy conversion could be increased, since the TMM operate with a difference in temperatures, without the requirement of high-pressure variations in the heat transfer fluid.

Another interesting potential that can be devised is that of applying thermomagnetic motors as an alternative to the Stirling engine in converting the heat energy produced by the fission nuclear reactor in a space environment. This application would demand additional applicability studies, but the theoretical calculations suggest that TMM could have a higher exergetic efficiency than the Stirling engines (VUARNOZ et al., 2012). This application has been made more relevant recently since NASA has published a report of a project to produce a $1 \mathrm{~kW}$ fission reactor using Stirling engines, to be used in space exploration (ANDERSON; WITTRY, 2018; KLOTZ, 2017). 


\section{Appendix 1 - Fabrication of the magnetic material plates}

Most of the promising magnetic materials to be used in thermomagnetic devices are brittle intermetallic compounds, as the MnAs, $\mathrm{La}(\mathrm{Fe}, \mathrm{Si})_{13}, \mathrm{Mn}_{3-\mathrm{x}} \mathrm{Fe}_{\mathrm{x}} \mathrm{Sn}$, and the $\mathrm{Gd}_{4.7} \mathrm{Nd}_{0.3} \mathrm{Si}_{4}$ compounds, causing them to be more easily obtained in a powder form than in a machinable stock form. One alternative is to bond the magnetic material powder with epoxy or other resin (PULKO et al., 2015; ZHU et al., 2018). This appendix presents an alternative way to bond magnetic material powders with an epoxy resin, using a pressure compaction technique. This work was presented in the conference "Danish Days on Caloric Materials and Devices" that took place at Roskilde, Denmark, on 2 and 3 of October of 2017 (FERREIRA et al., 2017).

The use of pressure compacting to bond the magnetic material powder and epoxy resin mixture facilitates the production of parts both for magnetic refrigeration and for thermomagnetic energy conversion systems. The method proposed is capable of obtaining magnetic material plates with complex geometries and internal channels for the flow of heat exchange fluids, like the ones used in the rotary thermomagnetic motor developed in this thesis.

The process begins with an ingot of the magnetic compound to be used, in this work the material $\mathrm{Gd}_{4.7} \mathrm{Nd}_{0.3} \mathrm{Si}_{4}$, which has a Curie temperature of $327 \mathrm{~K}$, with a second-order magnetic transition. This $\mathrm{Gd}_{4.7} \mathrm{Nd}_{0.3} \mathrm{Si}_{4}$ compound used in the motor was initially proposed by Coelho (GAMA, personal communication, September 25, 2017) based around the classic $\mathrm{Gd}_{5} \mathrm{Si}_{4}$, which has a $\mathrm{T}_{\mathrm{C}}$ of $336 \mathrm{~K}$ (PECHARSKY; GSCHNEIDNER, 2007). Coelho, based on a Scientific Initiation project, proposed the addition of Neodymium, with $\left(\mathrm{Gd}_{5-\mathrm{X}} \mathrm{Nd}_{\mathrm{X}}\right) \mathrm{Si}_{4}$ and $\mathrm{x}=0.3$, which reduces the $\mathrm{T}_{\mathrm{C}}$. The ingots were obtained through the arc melting technique, and heat treated at $1073.15 \mathrm{~K}$ for ten days. As this material presents a brittle and hard structure, it could not be easily machined through common fabrication processes.

The ingot was then turned into a fine powder using a ball milling machine; the powder was then sifted to a maximum gradation of $150 \cdot 10^{-6} \mathrm{~m}$. This powder was then thoroughly mixed with the epoxy resin, to allow the pressure compacting technique and to facilitate the handling of the material a high temperature curing resin was chosen. The MM and epoxy mixture were then poured into a pressure die mold, presented in Figure 86, and 
compacted using a hydraulic press to a force of 2.5 metric tons, with the pressure being kept for 2 minutes. After the compacting process, the part was ejected from the mold and put into a kiln at $413.15 \mathrm{~K}$ for 24 hours to complete the curing process.

Figure 86: The die pressure mold design shown in (a) is capable of producing magnetic material plates with complex geometry and internal channels for the flow of heat exchange fluids, as shown in (B). Dimensions are shown in millimeters

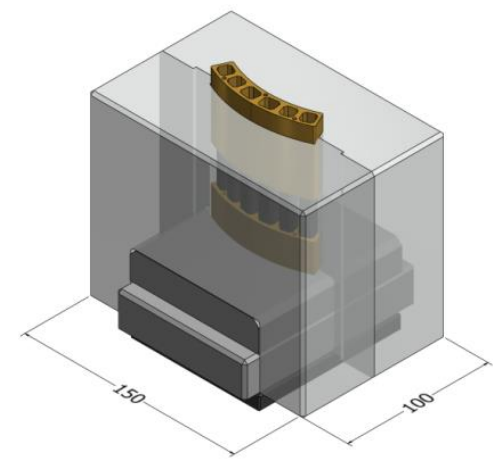

(a)

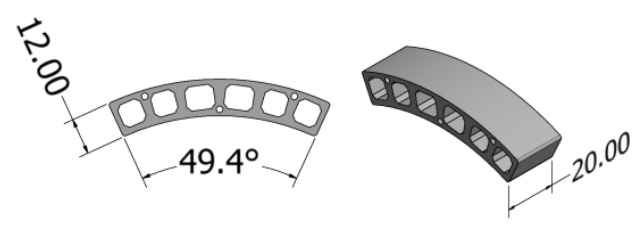

(b)

The design of the built mold, presented in Figure 87, takes into consideration the ejection of the compacted part, by providing adequate release angles and by using an adequate fine surface finish. Also, the pressure forces involved in the compacting forces were accounted for, with the mold being fabricated from tool steel. Due to the tolerances of the design, this mold had to be fabricated by a specialized tooling company.

Figure 87: Picture of the built pressure die mold with a $5 \times 5 \mathrm{~cm}$ scale reference. The mold was fabricated from tool steel by a specialized tooling company.

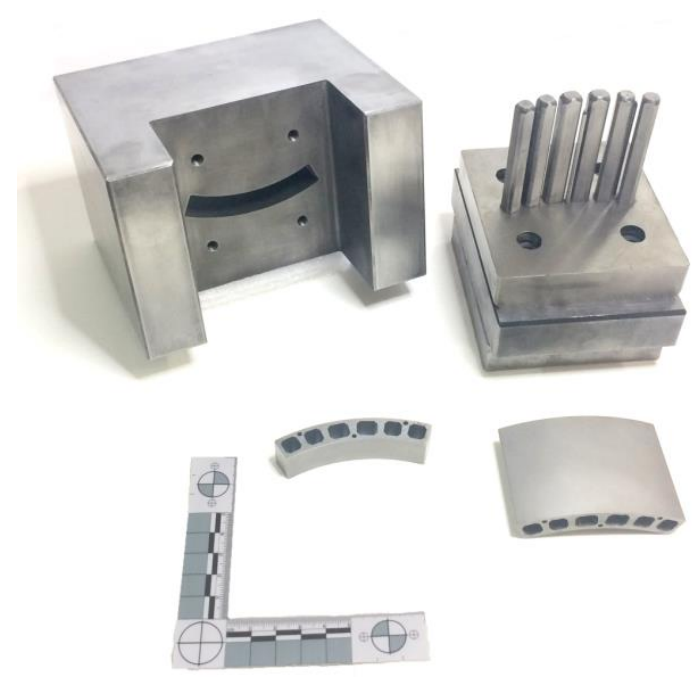

The length of the parts produced by this method was limited to the size of the mold used and also to difficulties in ejecting the compressed part from the mold. In order to 
produce plates of the desired length for the thermomagnetic motor developed, given a maximum length of $2 \cdot 10^{-2} \mathrm{~m}$ of the compressed part, as shown in Figure 86.b, the plates were produced in sections that were then connected by tungsten rods and glued using epoxy, the final plates produced are presented in Figure 88.

Figure 88: Picture of two of the plates produced by the compacting and epoxy bonding technique, they are composed of $20 \mathrm{~mm}$ sections (shown in Figure 86.b), connected with 3 tungsten rods and glued using epoxy, for a final length of $100 \mathrm{~mm}$.
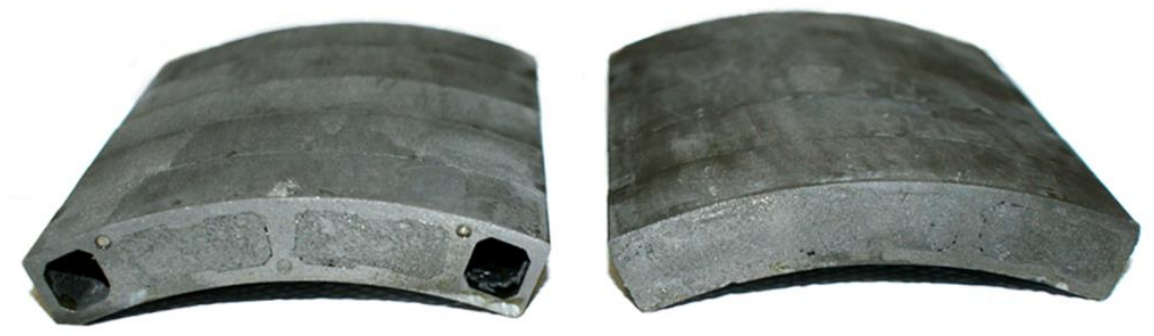

These plates have good mechanical and magnetic properties while maintaining a high ratio of magnetic material to epoxy, only 5\% in weight of epoxy is used to produce the plates, as opposed to previous works that have reported percentages of epoxy as high as $45 \%$ (WEISE et al., 2016).

The high pressure used helps to reduce the porosity of the finalized piece and eliminates the occurrence of unwanted bubbles or unfilled pockets. The density of the epoxy plates was determined by the buoyancy method to be $4.53 \mathrm{~g} / \mathrm{cm}^{3}$, presenting $66.51 \%$ of the density of the initial ingot of $6.81 \mathrm{~g} / \mathrm{cm}^{3}$. If the compacting force were increased from 2.5 metric tons to 3 metric tons, then the density of the final plate can be increased to $5.05 \mathrm{~g} / \mathrm{cm}^{3}$, $74.15 \%$ of the density of the initial ingot.

Given the proper design changes this technique can be used to produce plates of any format with the constant cross-section, or even plates with internal conical channels, that could improve the heat transfer with the flowing fluids (FERREIRA et al., 2014). 


\section{Appendix 2 - Thermal conductivity measurement apparatus}

In chapter 6 , the heat transfer on plates of the thermomagnetic motor is simulated using the CFD software Ansys CFX ${ }^{\circledR}$, among the many parameters that are necessary for such a simulation to represent reality, the thermal conductivity $\mathrm{k}$ of the materials used is of fundamental importance. While this parameter is usually very well defined, with extensive literature focused on reporting the measurements of $\mathrm{k}$ for a variety of conventional engineering materials (TOULOUKIAN et al., 1970a, 1970b), and fluids (TOULOUKIAN; LILEY; SAXENA, 1970), and also with many materials already built in the libraries of the software used (ANSYS INC., 2009). That is not the case for the material used in the fabrication of the plates of magnetic plates, because the thermomagnetic material used is composed of the uncommon intermetallic compound $\mathrm{Gd}_{4.7} \mathrm{Nd}_{0.3} \mathrm{Si}_{4}$, described in chapter 3, and also because this material is agglomerated with an epoxy resin under pressure. It became then necessary to measure the thermal conductivity of the used material to produce a simulation model capable of describing the heat transfer in the magnetic plates.

The transmission of heat in solids can happen via electrical carriers (electrons or holes), lattice waves (phonons), electromagnetic waves, spin waves, or other excitations. Usually, the total thermal conductivity $\mathrm{k}$ can be written as a sum of all the components representing various excitations $(\alpha)$, as defined by (YANG, 2005):

$$
k=\sum_{\alpha} k_{\alpha}
$$

The primary carriers of heat in metals are the electrons and lattice waves, which leads to a thermal conductivity given by:

$$
k=k_{e}+k_{g}
$$

In which $k_{e}$ is the electronic component and $k_{g}$ the lattice component. Usually, the lattice component of the thermal conductivity of metals, alloys, and semimetals is of magnitude comparable to the lattice thermal conductivity of insulators, except at cryogenic temperatures, where the phonon-electron interaction reduces the component $k_{g}$ in metals (TOULOUKIAN et al., 1970a). 
Therefore, the relative importance of the $k_{e}$ and $k_{g}$ terms depends on the magnitude of $k_{e}$, this electronic component of the thermal conductivity is often parallel to the electrical conductivity, following the Wiedemann-Franz law, given by:

$$
T \cdot L_{0}=\frac{k_{e}}{\sigma}
$$

With the ratio of the thermal conductivity $k_{e}$ and the electrical conductivity $\sigma$ directly proportional to the product of the temperature $\mathrm{T}$ and the Lorenz number $L_{0}\left(2.44 \cdot 10^{-8} \mathrm{~W} \cdot \Omega \cdot \mathrm{K}^{-}\right.$ ${ }^{2}$ ). Equation 3 has been experimentally validated for a wide variety of metals, ranging from pure metals to various alloys, showing that this proportionality is reasonably well obeyed at close to room temperature and above (KASAP, 2006).

It becomes then clear that there is a strong dependency of the thermal conductivity on the temperature of the material, not only because the $k_{e}$ changes with temperature, but also because the relevance of this change to the total conductivity will change. It is also important to notice that the lattice component may change with temperature, especially in the case where the material undergoes a phase change in the solid state, which is the case in the ferromagnetic to paramagnetic transition caused by the increase in the material temperature above the Curie temperature.

As an example of such change, the thermal conductivity of Nickel is presented in Figure 89, which undergoes a thermomagnetic transition at the temperature of $631 \mathrm{~K}$. The graph presents recommended reference values for the $99.99 \%$ pure Nickel, based on the critical evaluation of the validity of the available experimental data and related information, with the resolution of conflicting data by the correlation of results in terms of various affecting parameters, and comparison of the resulting values with the theoretical predictions. These recommended values are thought to be accurate to within $5 \%$ of the true values near room temperature, and 5 to $10 \%$ at other temperatures (TOULOUKIAN et al., 1970a). 
Figure 89: Nickel thermal conductivity as a function of the temperature, for a well-annealed $99.99 \%$ pure nickel, with residual electrical resistivity $\sigma_{0}=0.038410^{-4} \cdot \Omega \cdot \mathrm{m}$. The dotted vertical line shows the Curie temperature of the material at $631 \mathrm{~K}$

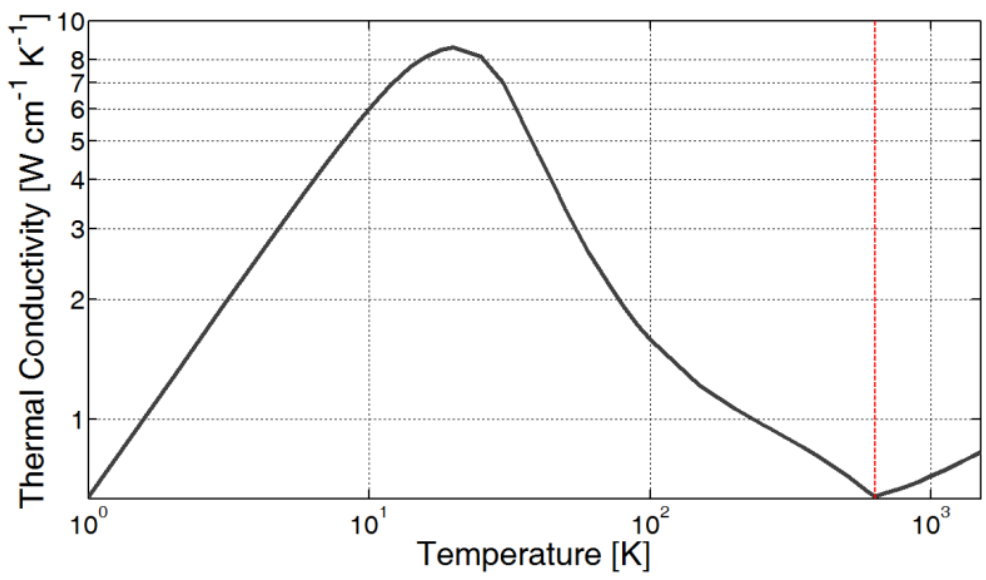

Source: Adapted from Touloukian et al. (1970a).

The graph presented in Figure 89 uses a $\log -\log$ scale, as is common practice in the reporting of thermal conductivity data. Although the log-log scales increase the apparent change for the higher temperatures, it is clear that the use of a single mean thermal conductivity value will not be representative of the real magnetic material behavior in the simulations, as it is cyclically heated and cooled around the Curie temperature. Therefore the thermal conductivity measurement must also provide values of the thermal conductivity for the change in the mean temperature the material is subjected to around the working temperatures of the simulated thermomagnetic motor.

Although there are thermal conductivity measurement equipment available in the market that could be used in the range of temperatures necessary, as the TA FOX 500 and TA FOX 50 (TA INSTRUMENTS, 2015), the NETZSCH LFA 467 (NETZSCH GERÄTEBAU GMBH, 2013), or the TCA 300 from TAURUS Instruments (TAURUS INSTRUMENTS, 2017). As these devices were not available, the research demanded the development of a small thermal conductivity measurement apparatus.

The measurement of the thermal transmission properties of materials can employ several methods, as different ranges of thermal conductivity values, different ranges of temperature and classes of materials require different techniques. The various methods of measurement of the thermal conductivity fall into two broad categories, the steady-state and the non-steady-state methods. In the first type, the specimen is subject to a time constant temperature profile, and the thermal conductivity is determined directly by measuring the 
heat flow rate per unit of area and the stable temperature gradient along the material. As for the non-steady state measurements, the temperature profile varies with time, and the measurement of the rate of the temperature change replaces the measurement of the rate of heat flow, in this case the thermal conductivity is derived from the thermal diffusivity measured, and a previous knowledge of the material's density and specific heat (TOULOUKIAN et al., 1970a).

The table presented in Figure 90 gives an overview of the applicability of different methods of measurement of the thermal conductivity, depending on the range of temperatures measured and the expected thermal conductivity range, although the values presented will have a strong dependency on the sample size and preparation.

Figure 90: Thermal Conductivity measurement device selection depending on the thermal conductivity range (with selected materials for comparison) and the measured temperature range.
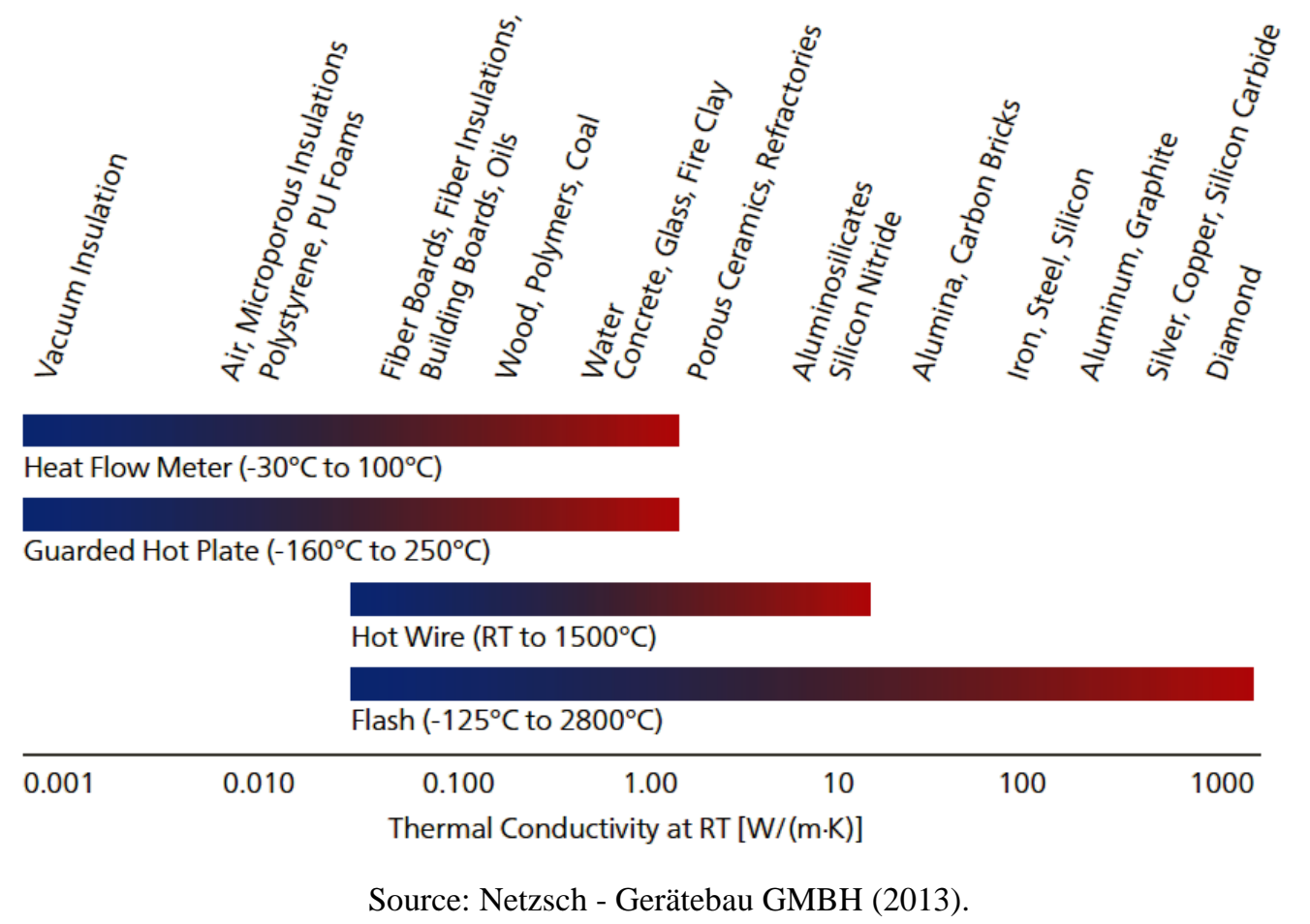

The heat flow meter under steady state method was chosen as the apparatus to be built, as it presents a relatively simple concept that applies to the range of temperatures necessary for this experiment. Despite the data presented in Figure 90, this method has been reported to work in the range from $78 \mathrm{~K}$ to $813 \mathrm{~K}$ (ASTM INT., 2017), and measuring thermal conductivities from 0.1 to $10 \mathrm{~W} \cdot \mathrm{m}^{-1} \cdot \mathrm{K}^{-1}$ with considerable precision (TA INSTRUMENTS, 2015). 
The orientations presented in the ASTM standard C518-17 (ASTM INT., 2017) guided the project of the measurement device, where the apparatus with one heat flux transducer was selected, as it presents the most straightforward construction, Figure 91 presents a schematic view of this design. The device consists of two isothermal temperature controlled plates, one heat flux transducer and equipment to control the ambient conditions when needed. According to the adopted standard, the isothermal plates must be assembled with sufficient rigidity and maintain good flatness and parallelism in all experimental conditions, the temperature of the plates must be measured by sensors mounted in grooves, as to be flush with the surface in contact with the specimen.

Figure 91: A standard test method for thermal conductivity measurement using a heat flow meter, apparatus with a single heat flux transducer and one specimen

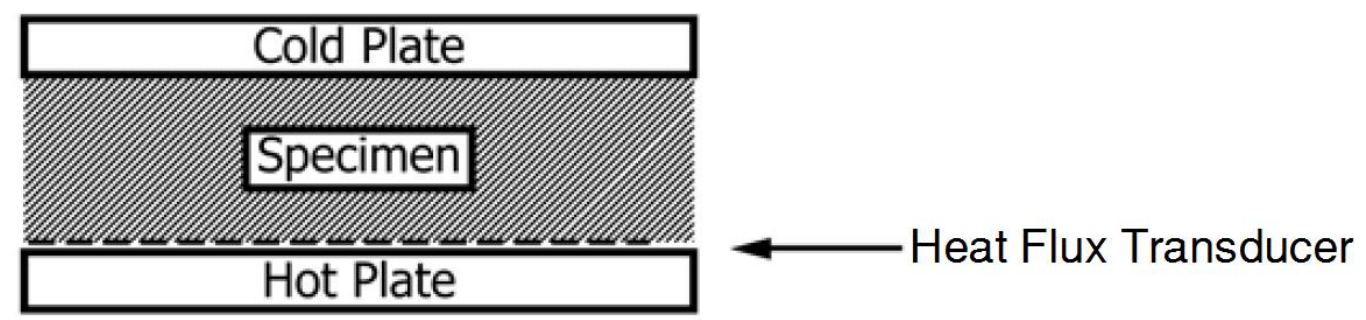

Source: ASTM INT. (2017).

In this system, the thermal conductivity is given by:

$$
k=\frac{Q \cdot l}{A \cdot \Delta T}
$$

In which the thermal conductivity $k$ is the total thermal conductivity observed, $Q$ is the heat flowing through the sample (W), $l$ is the length of the sample in the direction of the heat flow (m), $A$ is the cross-section area of the sample $\left(\mathrm{m}^{2}\right)$, and $\Delta T$ is the measured temperature difference across the sample $(\mathrm{K})$.

The precise determination of the $Q$ value is one of the main challenges presented in the heat flow meter method, as the input heat flow may present losses caused by radiation, heat conduction to the surrounding atmosphere or the sensors connecting leads. These losses cannot be eliminated entirely but may be minimized or accounted for through the use of appropriate experimental design (TRITT; WESTON, 2005). Also according to the ASTM standard C518-17 (ASTM INT., 2017) the significance of these, so-called Edge Losses, depends on the sample thickness, and proper edge insulation must be used to reduce this 
influence. Furthermore, any measured heat flow value will present an uncertainty relative to the adopted standard material used for the calibration of the heat flux transducer.

Another effect contributing to the uncertainty of the measured thermal conductivity of the material is thermal resistivity of the contact between the specimen and the isothermal plates; the apparent thermal resistivity $R_{A P}$ of the sample is given by:

$$
R_{A P}=\frac{l}{k}
$$

In which the $R_{A P}$ is the sum of the thermal resistance of the sample $R_{S}$ and the thermal resistance of the contacts $R_{C}$ in a given experiment. By making two measurements of the $R_{A P}$ with two samples of the same material and cross-section area A but different lengths one can estimate the thermal resistance of the contacts. With that the sample with smaller length will present a lower $R_{A P}$, by doing a first order linear fit between the two measurements of $R_{A P}$ and sample length $l$, one can then estimate the apparent resistance for a measurement of the thermal resistance of a sample with $l=0$. The resulting resistance will then be the thermal contact resistance $R_{C}$ of the system built, as the thermal resistance of the sample, in this case, would also be zero.

While this assessment of $R_{C}$ makes some questionable assumptions, as that there is perfect repeatability in the mounting process of the experiments with samples of different lengths and that the estimated thermal contact resistance will be similar for experiments realized with different materials. This approximation shows that by realizing a proper specimen preparation and experimental procedures, and if the material measured has a sufficiently high thermal resistance, the influence of the $R_{C}$ in the experimental uncertainty should be smaller than the other uncertainties associated with the experimental procedure.

It is important to notice that, according to Tritt and Weston, (2005), even if one can minimize or effectively measure the previous uncertainties, the accurate determination of the sample length $l$, and the cross-sectional area $A$ can be a challenge to achieving high precision in the determination of the thermal conductivity within a 5-10\% uncertainty.

The project of the measurement device focused on producing a system flexible and sufficiently accurate. While also being easy to build, operate, and capable of producing the necessary curve of the thermal conductivity as a function of the temperature, in the range applied in the motor (from room temperature up to $348 \mathrm{~K}$ ). The heat flux across the sample, 
needed to perform the measurement, was produced by two Peltier elements operating as heat pumps or active heat sinks. This design uses commercially available Peltier elements, as they are found to combine the adequate flexibility of control and sensitivity, being able to operate at up to $373 \mathrm{~K}$ and to cool down to cryogenic temperatures, provided the appropriate heat sinking. The Peltier plates chosen were two TEC1-12706 modules (THERMONAMIC MODULE, 2017), with 40x40x3.8 mm and rated to work with up to $12 \mathrm{~V}$ and a cooling capacity of $60 \mathrm{~W}$.

It is essential that the applied thermal gradient reaches a steady state condition before any measurement; therefore the cooling/heating power in these Peltier elements must also be kept in a steady state condition for each sampling point. This is done by operating the elements at constant electrical power, using linearized and stable voltage sources, and also by keeping a constant temperature at the heat sinking face of elements. By applying a different power to each element the thermal gradient is kept along the isothermal plates and the sample.

A Peltier effect element can also be used as a heat flux transducer, as they can operate as a thermopile made of a material with an optimized thermoelectrical figure of merit (PLACKOWSKI; WANG; JUNOD, 2002). Forcing a heat flow $Q_{P}$ across the Peltier element results in a temperature difference $\Delta T_{P}$ given by:

$$
\Delta T_{p}=\frac{Q_{p}}{k_{p}}
$$

Between the two faces of the Peltier plate, and also a voltage $V_{P}$ across the element terminals, given by:

$$
V_{p}=S_{P} \cdot \Delta T_{p}
$$

In which $S_{P}$ is the Seebeck coefficient for the Peltier element. This way, the sensitivity of the Peltier element can be defined by the constant $A_{P}\left(\mathrm{~V} \cdot \mathrm{W}^{-1}\right)$ as:

$$
A_{p}=\frac{V_{p}}{Q_{p}}=\frac{S_{p}}{k_{p}}
$$

The sensitivity of the Peltier element $A_{P}$ is dependent on the temperature, and therefore this constant must be adequately calibrated for the element used. Despite this fact, these devices have a high potential to be used as heat flow transducers, presenting an excellent signal to 
noise ratio (PLACKOWSKI; WANG; JUNOD, 2002) and sensitivity. The Peltier element to be used was similar to the CP10-71-06 (LAIRD TECHNOLOGIES, 2017) with $23 \times 23 \times 3.6 \mathrm{~mm}$. Initial tests with this element showed that when in equilibrium with room temperature it is capable of detecting the heat flow provided by the flash of a red laser diode (class IIIa, $5 \mathrm{~mW}, 650 \mathrm{~nm}$ ).

For the measurement device proposed, with the design presented in Figure 92, a cylindrical shaped specimen, with a diameter of $11 \mathrm{~mm}$, is used as the material sample. The two isothermal plates are made of aluminum, and have a shape similar to that of the specimen, with small groves for the $\mathrm{N}$-type thermocouples used as temperature sensors $\left(T_{C-1}\right.$ and $T_{C-2}$ ). Two water-cooled heat sinks are used to remove the heat from the heat pumping Peltier elements; these heat sinks are mounted into aluminum support bases, connected by threaded rods, allowing for the measurement of the conductivity of samples with varying thicknesses. Two other N-type thermocouples are attached to the systems, one in the Peltier working as heat flux transducer, and another in the opposing heat pumping Peltier element (Hot Side Peltier element).

Figure 92: Proposed design of the thermal conductivity measurement apparatus.

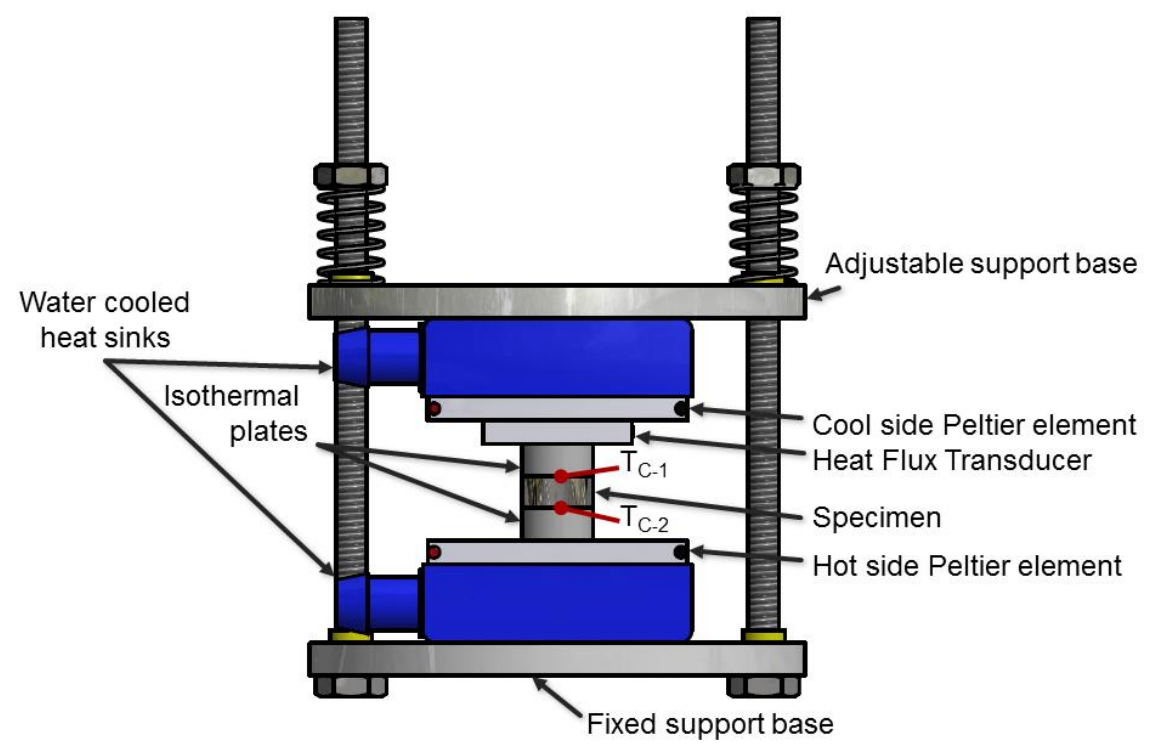

As previously noted, the contact thermal resistance might present an uncertainty hard to account for in the measurements, because of that, silver filled thermal conductive glue is used in order to reduce the thermal contact resistance in the fixed parts. As for the contact between the surface of the specimen and the isothermal plates, the surfaces of the isothermal 
plates are polished to a mirror finish, and also the specimens must be polished and heat conducting grease applied as part of the experimental procedure. The adjustable support base (Figure 92) compresses the specimen, helping to reduce any air gaps that may be present in the contact between the specimen and isothermal plates. The compression is done by the force produced by four springs in the threaded rods, the use of the springs allows the control of the compression force applied and also help to account for any non-parallelism that may be present in the specimen measured.

The heat conduction from the specimen and isothermal plates to the surrounding atmosphere constitutes another critical uncertainty in the measurements; this heat conduction will influence in the measured heat flux $Q$, which is expected to be unidirectional along the thickness of the specimen. In the experiments presented only specimens with thicknesses inferior to their radius are used, and most importantly, the system is mounted inside a vacuum chamber, and the measurements only realized with a maximum allowable pressure of 0.4 Torr. In practice this eliminates the influence of heat conduction to air, the remaining undesirable heat conduction will occur due to radiation, which is expected to be very low as the maximum temperatures in the experiment are relatively close to room temperature, and due to the unavoidable heat conduction to the connecting leads of the temperature sensors.

Figure 93 presents a picture of the built thermal conductivity measurement device, with a reference scale. The construction followed the design proposed in Figure 92; only the heat flux transducer is moved to the fixed side of the support base, to facilitate the assembly. The water-cooled heat sinks are connected to hoses circulating water at room temperature. The measurement apparatus is mounted inside a vacuum chamber, which has its internal pressure measured by a PKR 251 full range vacuum sensor (PFEIFFER VACUUM, 2005) that is connected to the Adixen ACM 2000 digital indicator (ALCATEL VACUUM TECHNOLOGY, 2014). Allowing the pressure inside the vacuum chamber to be continuously monitored during the experiments. 
Figure 93: Picture of the built thermal conductivity measurement apparatus, with a $5 \mathrm{~cm}$ scale.

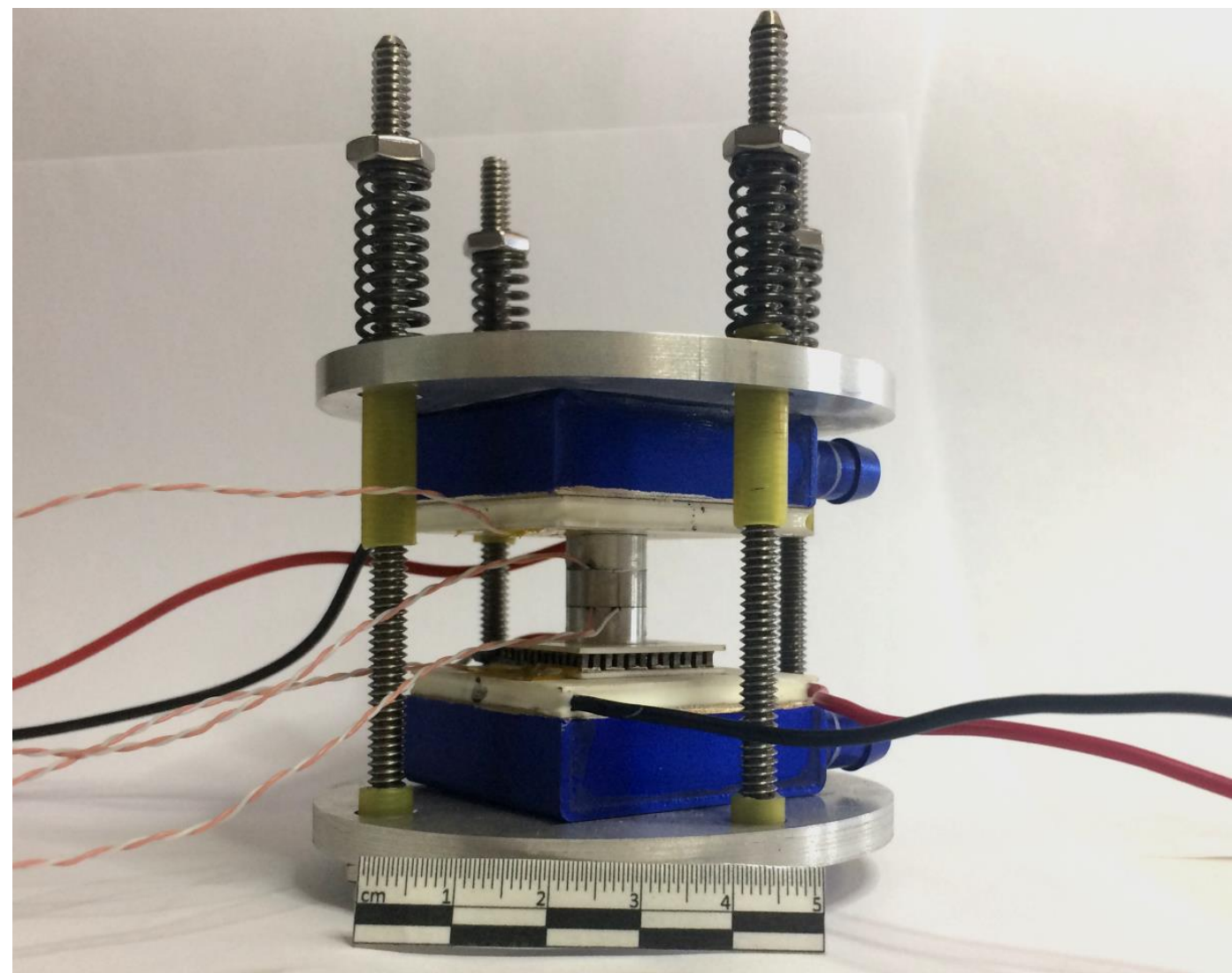

The data acquisition in the system is realized by the use of an Arduino Mega 2560 board (ARDUINO LLC, 2010), this board is based around the microcontroller ATmega2560, and presents 54 digital I/O lines and 16 analog input pins, with an ATmega16U2 IC used for the USB communication with a PC. The use of these boards as inexpensive data acquisition systems in the laboratory have already demonstrated promising results (D’AUSILIO, 2012), and proved to be adequate for the built measurement apparatus.

Although the ATmega2560 presents 16 analog input pins, the internal analog-to-digital converter (ADC) is limited to 10 bits of resolution, which would not allow the reading of the thermocouples or the heat flux transducer outputs with the desired precision. Two IC's are used to overcome this resolution problem. The MAX31856 is used as the thermocouple to digital converter (MAXIM INTEGRATED, 2015), this IC provides high-accuracy thermocouple temperature readings, including automatic linearization correction for 8 thermocouple types (including the $\mathrm{N}$-types used in this project) and a 19-bits resolution, which provides a thermocouple reading resolution of $0.0078 \mathrm{~K}$, also containing 
internal Cold-Junction compensation, noise rejection filters and overvoltage protection, with the digital temperature reading communicated to the ATmega2560 via the $\mathrm{I}^{2} \mathrm{C}$ serial protocol. The second IC is the ADS1115 16-Bits ADC (TEXAS INSTRUMENTS, 2009) used to read the heat flow transducer signal, this IC offers a programmable gain amplifier of up to $16 \mathrm{x}$, allowing the resolution to be applied to a smaller range, and also communicating the readings via the $I^{2} \mathrm{C}$ protocol. In the system built a $2 \mathrm{x}$ gain is used, with a maximum reading of $2.048 \mathrm{~V}$ and a resolution of $0.0625 \mathrm{mV} \cdot \mathrm{Bit}^{-1}$, the IC is also configured to operate a differential voltage reading between the two leads of the heat flux transducer, providing readings of heat flux in two directions.

As the sensors readings are communicated to a PC via USB, the signal processing, and the data recording are done using the VISA communication with the LabVIEW Software. With this platform, a very flexible and fast data reading and processing are possible, as it is not necessary to reprogram the data acquisition Arduino Mega 2560 for any change in the processing of the readings.

It is essential to calibrate the built apparatus to obtain an adequate reading of the heat flow $Q$ with a known uncertainty, obtaining the sensitivity of the Peltier used as heat flow transducer as a function of the applied temperature $A_{p}(T)$. Therefore it is necessary to adopt a standard calibration material, with known thermal conductivity in the measured range. The standard chosen was the 304 stainless steel, which is recommended for measurement of $k$ below $20 \mathrm{~W} \cdot \mathrm{m}^{-1} \cdot \mathrm{K}^{-1}$ (TRITT; WESTON, 2005), and also presents a good stability in the range of measured temperatures. The thermal conductivity as a function of the temperature of the standard 304 stainless steel is presented in Figure 94. 
Figure 94: AISI 304 stainless steel recommended thermal conductivity as a function of the temperature, the estimated error is of $\pm 10 \%$ below $100 \mathrm{~K}, \pm 5 \%$ for the range from 300 to $800 \mathrm{~K}$, and $\pm 10 \%$ above $1400 \mathrm{~K}$.

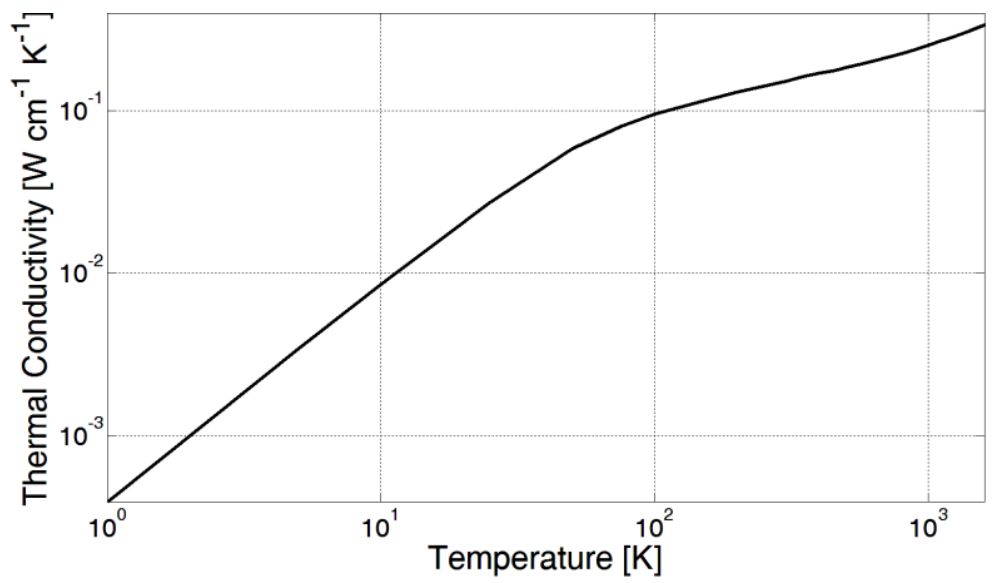

Source: Adapted from Touloukian et al. (1970a).

The preparation procedure of the calibration sample consisted in the fabrication of a cylinder of 304 stainless steel with a diameter of $10.89 \pm 0.01 \mathrm{~mm}$ and a thickness of $4.55 \pm 0.02 \mathrm{~mm}$., the flat faces of the sample were polished using a metallographic polishing machine, with 1-micron aluminum oxide as the final polishing abrasive, and obtaining a mirror finish. The sample was then mounted in the built apparatus, and the apparatus closed inside the vacuum chamber, which was then evacuated to a pressure of $0.23 \pm 0.01$ Torr, the pressure of the chamber is monitored and kept constant during the whole experiment.

The fundamental condition of steady state heat flow, for the calculation of the thermal conductivity, and the application of equation 4, is observed for both the measured data and the calibration of the heat flow transducer. This condition is guaranteed by observing the stability of the measured heat flow (through the voltage developed in the transducer), the temperatures across the sample, and the cooling power applied by the heat pumping Peltier elements. The data processing software running in LabVIEW calculates the point-by-point derivative of those signals in time intervals of $500 \mathrm{~ms}, 5 \mathrm{~s}, 1 \mathrm{~min}$, and $10 \mathrm{~min}$. These calculations provide the stable development of the heat flow as the value of the derivatives approach zero. The use of short and long term interval is necessary to filter any random noise, which would appear as short time instabilities, while also accounting for the large time constants involved in the processes of heat transfer, which appear as long term instabilities. The data acquisition software will then only write the measured values when all signals observed are stable, this process can take from $30 \mathrm{~min}$ to 2 hours for each measurement point, depending on the material, and the measured temperatures and applied heat flow. 
The determination of the heat flow across the sample is done by the calculation of the mean temperature across the sample (mean of $\mathrm{T}_{\mathrm{C}-1}$ and $\mathrm{T}_{\mathrm{C}-2}$ ), and the evaluation of the reference thermal conductivity in this temperature $\left(\mathrm{K}_{\mathrm{Ref}}\right)$, based on the interpolant fitting of the data presented in Figure 94. Then the corresponding reference heat flow $\left(\mathrm{H}_{\mathrm{Ref}}\right)$ can be calculated using Equation 4. As the voltage across the Peltier element acting as heat flux transducer is logged, the corresponding Peltier sensitivity of this element can be obtained using Equation 8. Although this procedure can be adopted with good results by the measurement of the Peltier heat flow sensitivity with varying temperatures (PLACKOWSKI; WANG; JUNOD, 2002). It does not account for the correlation between the heat flow and the temperature of Peltier element, due to the variation in the Peltier thermal conductivity $\mathrm{k}_{\mathrm{p}}$, which will be more relevant above room temperature. Therefore a more accurate calibration of the heat flow sensitivity can be done by the least squares interpolation of the reference heat flow, the Peltier temperature and the measured Peltier voltage.

The calibration interpolant can be used to determine the heat flow across the transducer given the Peltier temperature and voltage. In order to obtain a good fit 25 measurements were made, with varying temperatures and for different heat flow conditions, changing not only the intensity of the $\mathrm{H}_{\text {Ref }}$ but also its direction, which helps to provide a reasonable fitting of results even for conditions of low $\mathrm{H}_{\text {Ref }}$, although the influence of errors is expected to be higher for such conditions. The resulting fitted curve is presented in Figure 95.

The main error contributions in the fitted data are provided by the calculated $\mathrm{H}_{\mathrm{Ref}}$, as it depends on reference conductivity of the 304 stainless steel, that is provided with a known uncertainty of $\pm 5 \%$, which is compounded by the linear interpolant fitting of the data with the mean temperature of the sample, with an expected uncertainty of $0.87 \mathrm{~K}$ for temperatures close to room temperature, given mainly by intrinsic uncertainty of the thermocouples used. The influence of sample dimension errors, as well as of mounting misalignments and contact resistances between the sample and the isothermal plates are expected to be less significant, as great care was taken in the sample preparation process and experiment assembly. 
Figure 95: Fitting of the calculated reference heat flow with the Peltier Temperature and Peltier Voltage, using the least squares interpolant, the black dots indicate the measured data points.

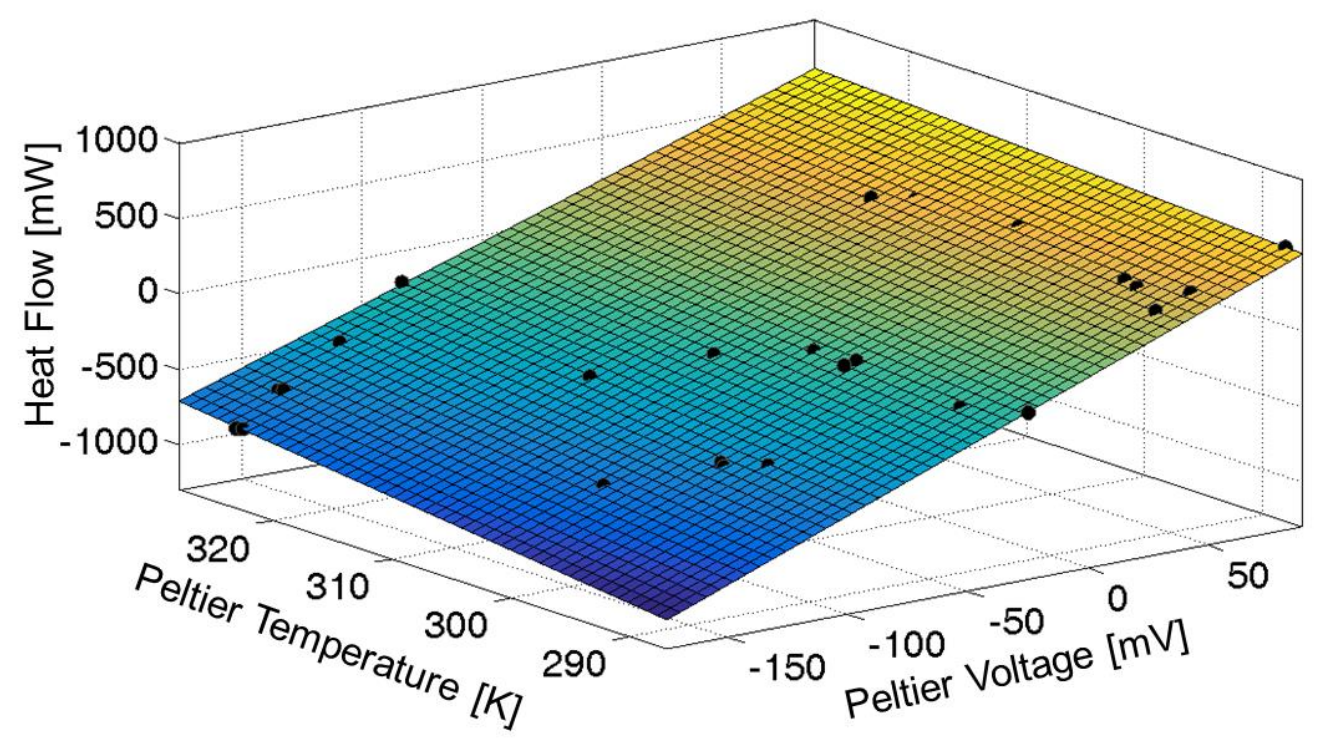

A first evaluation of the calibration of the heat flow transducer, and of the built thermal conductivity apparatus as a whole, is done by the comparison of the measured thermal conductivity of the 304 stainless steel used in the calibration process. A different set of 10 data points is used, allowing the cross-validation of the calibration data, as shown in Figure 96. In the figure the reference data is presented with the uncertainty bounds of $\pm 5 \%$ (dotted lines). As shown most of the measured data is close to thermal conductivity reference data, and even the most divergent data points are still within the uncertainty bounds of the reference data. 
Figure 96: Evaluation of the thermal conductivity of 304 stainless steel measured data compared to the known reference values from Touloukian et al. (1970a).

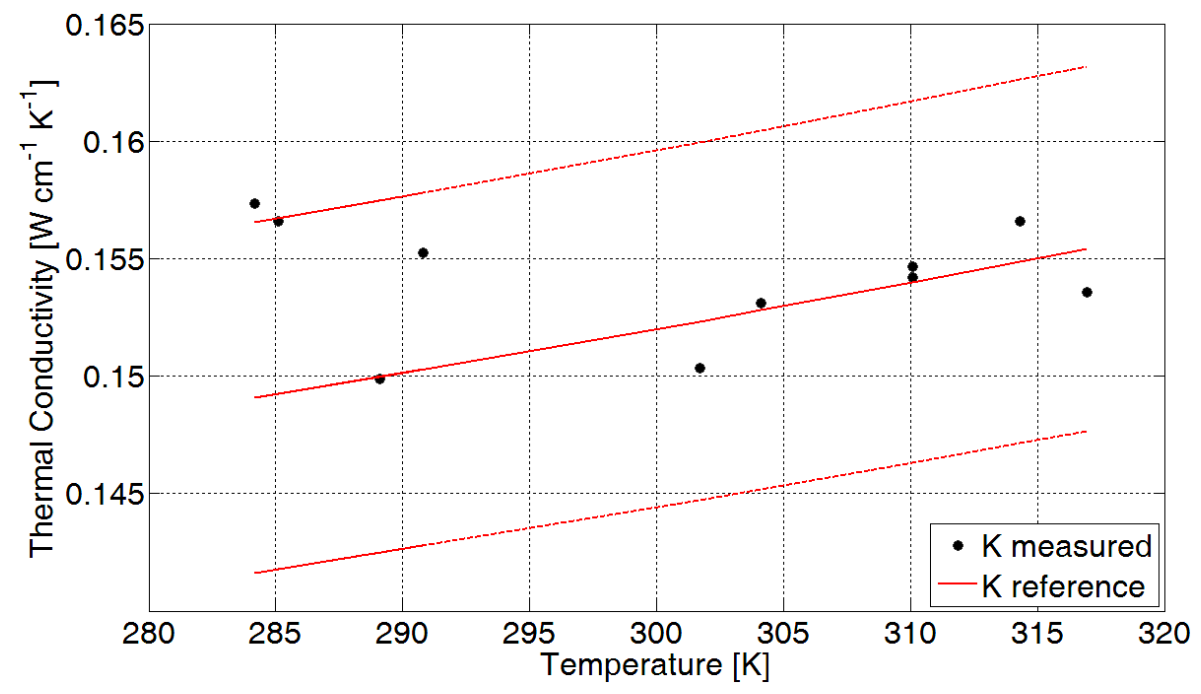

A more through validation of the calibration of the thermal conductivity measurement system can be obtained by the comparison of the measurement data with that obtained for another class of materials, different from the one used in the calibration procedure. This allows the test of the validity and limitations of the system. As the system is aimed at measuring the thermal conductivity of the intermetallic compound $\mathrm{Gd}_{4.7} \mathrm{Nd}_{0.3} \mathrm{Si}_{4}$ as agglomerated with an epoxy resin, which is expected to have a low thermal conductivity, it is therefore interesting to test a material with a thermal conductivity lower than that of the reference material used for calibration. Therefore a titanium alloy was chosen as good test material, as it is easily machinable in the desired sample format and has a good corrosion resistance.

The alloy chosen was the Ti-6Al-4V (also referred to as TC4), due to its availability and also to the potential of having a thermal conductivity almost an order of magnitude lower than that of the 304 stainless steel used in the calibrations of the system. Although there is no recommended reference thermal conductivity data for this alloy present in the researched literature, Touloukian et al. (1970a) presents a series of reference measurements for this alloy from their collected measurements within the Titanium-Aluminum- $\sum \mathrm{X}_{\mathrm{i}}$ alloys category, from which three measurements are closer to the range of temperatures achieved in the designed apparatus. The reference data, as well as the measured $\mathrm{k}$ are presented in Figure 97. 
Figure 97: Evaluation of the thermal conductivity data measured for the Ti-6Al-4V titanium alloy, and comparison between the measured data and the available references from Touloukian et al. (1970a).

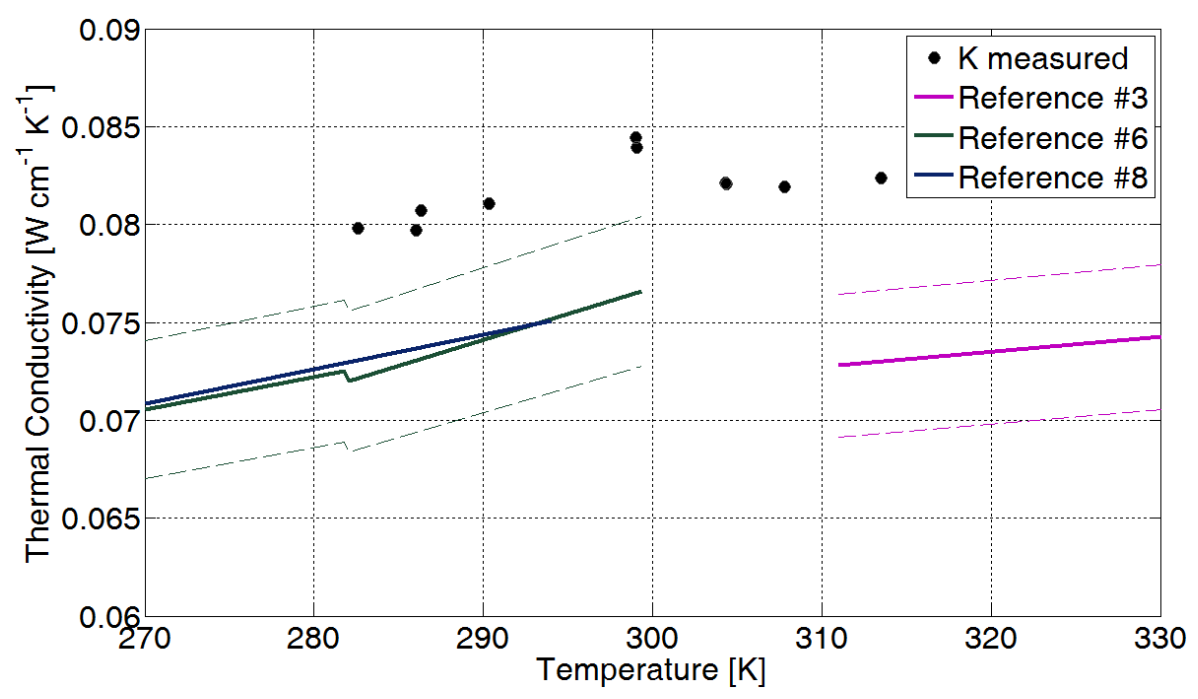

The results presented in Figure 97 indicate that even for lower thermal conductivities the measured values of $\mathrm{K}$ can be considered close to the values presented in the reference literature. It is interesting to notice that the references did not report values in the exact range of the measurements done using the developed apparatus. Although the value of the measured thermal conductivity shown in Figure 97 seems to have a tendency of being higher than the reference values, the limits of the uncertainty in the measured data are well within that of the references, and these differences may be accounted for in variations in the sample composition and preparation that could not be adequately addressed.

As the system developed could be proved to obtain good results in the range of temperatures desired to simulate the behavior of the thermomagnetic motor, the measurement of the thermal conductivity of the intermetallic compound $\mathrm{Gd}_{4.7} \mathrm{Nd}_{0.3} \mathrm{Si}_{4}$ as agglomerated with epoxy resin was finally done. To obtain a sample that could adequately represent the state of the material in the plates used in the motor, a plate was fabricated with the same composition and proportion of $\mathrm{Gd}_{4.7} \mathrm{Nd}_{0.3} \mathrm{Si}_{4}$ and $5 \%$ in weight of Epoxy, and compacted in the mold used to develop the plates used in the motor. Then this plate was machined, using abrasive sculpting, into a cylinder with a diameter of $11.05 \pm 0.05 \mathrm{~mm}$ and a thickness of $1.10 \pm 0.1 \mathrm{~mm}$.

The measured thermal conductivity of the material used in the plates of the thermomagnetic motor is presented in Figure 98, the data is presented with its expanded uncertainty, which is calculated based on the compounded uncertainty of the calibration and 
measured data using the Monte Carlo implementation of the Guide for Uncertainty in Measurements (JOINT COMMITTEE FOR GUIDES IN METROLOGY, 2008b) and run within MATLAB® (FERNÁNDEZ; CALDERÓN; DÍEZ, 2009). A curve is fitted to the data using the smoothing spline method, using the uncertainty as the fitting weight of the data.

Figure 98: Measured thermal conductivity of the intermetallic compound $\mathrm{Gd}_{4.7} \mathrm{Nd}_{0.3} \mathrm{Si}_{4}$ as agglomerated with $5 \%$ wt. of epoxy resin, and the fitted curve used in the CFD simulations.

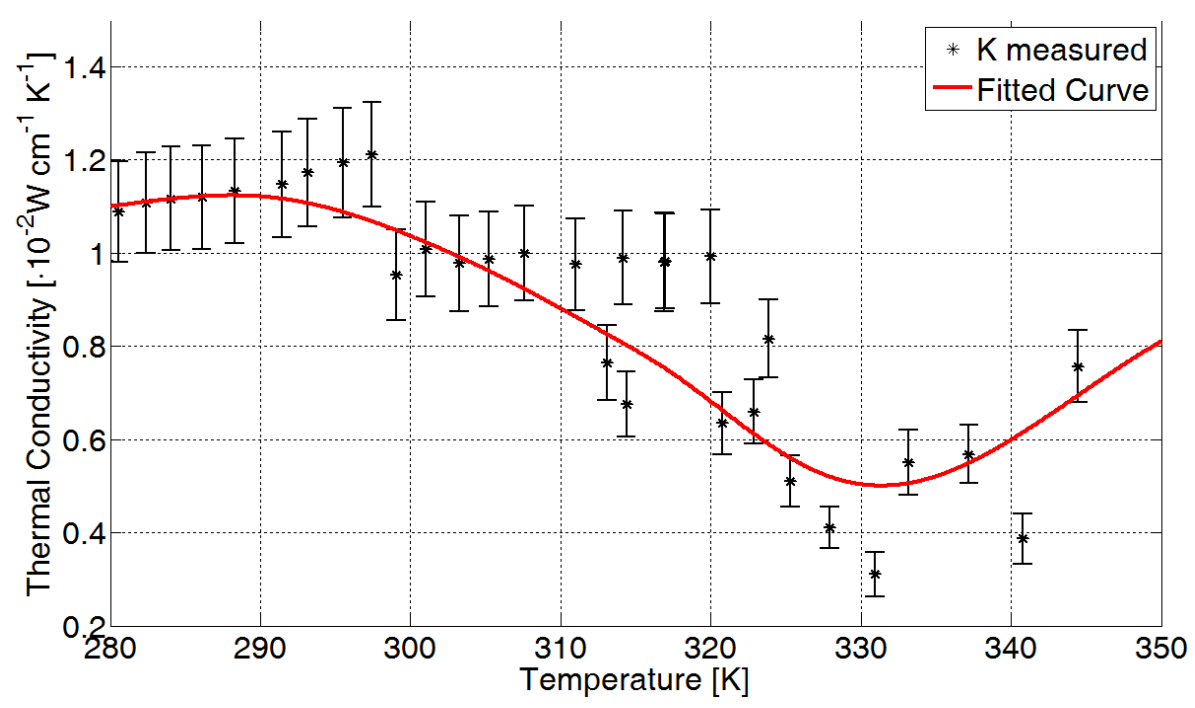

The fitted curve data can therefore be used to simulate the thermal conductivity behavior of the studied material in the CFD simulation of the motor. It is also interesting to notice that as shown for the Nickel in Figure 89, the thermal conductivity of the compound $\mathrm{Gd}_{4.7} \mathrm{Nd}_{0.3} \mathrm{Si}_{4}$ presents an inflection point close to the Curie temperature (which is expected to be around $327 \mathrm{~K})$.

Many improvements can be devised for this simple apparatus, which could lead to more precise and reliable data. In special the substitution of the thermocouples for more adequate thermistors could greatly improve these results. Another important point would be to use current sources with a more stable output for the Peltier elements used as heat pumps, which could also be substituted for electrical resistances capable of reaching higher temperatures than the ones reported here. Despite these facts, the system built was capable of producing meaningful results and the desired data was obtained. An interesting spin-off from this research could be the development of measurements of the thermal conductivity for different thermomagnetic materials with potential to be applied in motors or in magnetic refrigeration, as these data are not commonly available in the literature and are of fundamental importance for the development of simulations as well as practical applications. 


\section{Bibliography}

AGÊNCIA NACIONAL DE ENERGIA ELÉTRICA. Atlas de Energia Elétrica do Brasil. [s.l: s.n.].

ALCATEL VACUUM TECHNOLOGY. Adixen vacuum measurement solutions Technology, 2014. Disponível em: <http://www.idealvac.com/files/literature/17_Pfeiffer_Adixen_Alcatel_2000_Series_Vacuu m_Gauges.pdf>.Acesso em: 12 fev. 2018

ALFA INSTRUMENTOS. Célula de Carga - S. Disponível em: <http://www.alfainstrumentos.com.br/produto/s/>. Acesso em: 20 dez. 2017.

ALMANZA, M. et al. Numerical study of thermomagnetic cycle. Journal of Magnetism and Magnetic Materials, v. 426, p. 64-69, mar. 2017.

ALVES, C. S. et al. Numerical simulation and design of a thermomagnetic motor. Applied Thermal Engineering, v. 61, n. 2, p. 616-622, 2013.

ALVES, C. S. et al. Simulation of solar Curie wheel using NiFe alloy and Gd. International Journal of Refrigeration, v. 37, n. 1, p. 215-222, 2014.

ANDERSON, G.; WITTRY, J. Demonstration Proves Nuclear Fission System Can Provide Space Exploration Power. Disponível em: <https://www.nasa.gov/pressrelease/demonstration-proves-nuclear-fission-system-can-provide-space-exploration-power $>$. Acesso em: 28 jun. 2018.

ANSYS. ANSYS CFX Introduction CanonsburgAnsys Inc., , 2013. Disponível em: $<$ http://itrss.mst.edu/media/informationtechnology/itrss/documents/ansysdocs/ANSYS CFX Introduction.pdf>

ANSYS INC. Ansys CFX-Pre User's Guide Ansys Inc., , 2009.

ARDUINO LLC. Arduino MEGA 2560. Disponível em: <https://www.arduino.cc/en/Main/ArduinoBoardMega2560/>. Acesso em: 12 fev. 2018.

ASTM INT. C518-17: Standard Test Method for Steady-State Thermal Transmission Properties by Means of the Heat Flow Meter Apparatus ASTM International, 2017. Disponível em: 〈http://www.astm.org/cgi-bin/resolver.cgi?C518-17>

BASTIAN, M.; HEYMANN, S.; JACOMY, M. Gephi: An Open Source Software for Exploring and Manipulating Networks. Third International AAAI Conference on Weblogs and Social Media, p. 361-362, 2009.

BERLINER, E. Electric Furnace Generator United States, 1892. Disponível em: <https://patents.google.com/patent/US481999A/>

BESSA, C. V. X. et al. On the influence of thermal hysteresis on the performance of thermomagnetic motors. Journal of Applied Physics, v. 122, n. 24, p. 244502, 28 dez. 2017.

BJØRK, R. et al. An optimized magnet for magnetic refrigeration. Journal of Magnetism and Magnetic Materials, v. 322, n. 21, p. 3324-3328, nov. 2010. 
BJØRK, R.; BAHL, C. R. H.; INSINGA, A. R. Topology optimized permanent magnet systems. Journal of Magnetism and Magnetic Materials, v. 437, n. October 2016, p. 7885 , set. 2017.

BOX, G. E.; HUNTER, J. S.; HUNTER, W. G. Statistics for experimenters: design, innovation, and discovery. 2. ed. [s.1.] Wiley-Interscience New York, 2005.

BOZORTH, R. M. Ferromagnetism. [s.1.] IEEE Press, 1978.

BRAILSFORD, F. Theory of a ferromagnetic heat engine. Proceedings of the Institution of Electrical Engineers, v. 111, n. 9, p. 1602, 1964.

BREMER, H. Electrical heat-motor. USA, 1904. Disponível em: <https://www.google.com/patents/US764518>

BRILLOUIN, L.; ISKENDERIAN, H. P. Thermomagnetic Generator. Electrical Communication, v. 25, n. 3, p. 300-311, 1948.

CANEVA, K. L. "Discovery" as a site for the collective construction of scientific knowledge. Historical Studies in the Physical and Biological Sciences, v. 35, n. 2, p. 175291, 2005.

CARLSON, W. B. Tesla: Inventor of the Electrical Age. New Jersey: Princeton University Press, 2013.

ÇENGEL, Y. A. Heat and Mass Transfer: A Practical Approach. p. 901, 2007.

CHAMPIER, D. Thermoelectric generators: A review of applications. Energy Conversion and Management, v. 140, p. 167-181, 2017.

CHEN, C. C. et al. A Miniature Magnetic-Piezoelectric Thermal Energy Harvester. IEEE Transactions on Magnetics, v. 51, n. 7, 2015.

CHEN, H.; GOSWAMI, D. Y.; STEFANAKOS, E. K. A review of thermodynamic cycles and working fluids for the conversion of low-grade heat. Renewable and Sustainable Energy Reviews, v. 14, n. 9, p. 3059-3067, 2010.

CHRISTIAANSE, T.; BRÜCK, E. Proof-of-Concept Static Thermomagnetic Generator Experimental Device. Metallurgical and Materials Transactions E, v. 1, n. 1, p. 36-40, 22 mar. 2014.

CHU, S.; MAJUMDAR, A. Opportunities and challenges for a sustainable energy future. Nature, v. 488, n. 7411, p. 294-303, 2012.

CHUN, J. et al. Thermo-Magneto-Electric Generator Arrays for Active Heat Recovery System. Scientific Reports, v. 7, n. August 2016, p. 1-8, 2017.

COELHO, A. DE A.; GAMA, S.; SILVA, I. DA. Dispositivo termomagnético rotativo e uso do mesmo Brasil, 2012. Disponível em: <https://gru.inpi.gov.br/pePI/servlet/PatenteServletController?Action=detail\&CodPedido=90 7035\&SearchParameter=BR1020120128241 \&Resumo=\&Titulo=>

COLEMAN, H. W.; STEELE, W. G. Experimentation, validation, and uncertainty analysis for engineers. Third Edit ed. [s.1.] John Wiley \& Sons, 2009.

CURIE, P. Propriétés magnétiques des corps à diverses températures. [s.1.] Gauthier- 
Villars ( Paris(France) ), 1895.

CURIE, P. Euvres de Pierre Curie: publiées par les soins de la Société Française de Physique. Paris: Société Française de Physique, 1908.

D'AUSILIO, A. Arduino: A low-cost multipurpose lab equipment. Behavior Research Methods, v. 44, n. 2, p. 305-313, 25 jun. 2012.

DAN'KOV, S. et al. Magnetic phase transitions and the magnetothermal properties of gadolinium. Physical Review B, v. 57, n. 6, p. 3478-3490, 1998.

DENHOLM, P. et al. Overgeneration from Solar Energy in California. A Field Guide to the Duck Chart National Renewable Energy Laboratory. Golden, CO (United States): [s.n.]. Disponível em: <https://www.nrel.gov/docs/fy16osti/65023.pdf>.

EDISON, T. A. On the Pyromagnetic Dynamo - A Machine for Producing Electricity Directly from Fuel. The Electrical World, v. X, n. 9, p. 111-113, 1887a.

EDISON, T. A. On the Pyromagnetic Dynamo - A Machine for Producing Electricity Directly from Fuel. SCIENTIFIC AMERICAN, v. 57, n. 9, p. 113, 1887b.

EDISON, T. A. Pyromagnetic Motor United States, 1888. Disponível em: <https://www.google.com/patents/US380100>

EDISON, T. A. Pyromagnetic Generator USA, 1892. Disponível em: <https://patents.google.com/patent/US476983A>

EGOLF, P. W. et al. Magnetic power conversion with machines containing full or porous wheel heat exchangers. Journal of Magnetism and Magnetic Materials, v. 321, n. 7, p. 758-762, 2009.

ELliOTT, J. F. Thermomagnetic generator. Journal of Applied Physics, v. 30, n. 11, p. 1774-1777, 1959.

FARADAY, M. Experimental Researches in Electricity. Twenty-First Series. Philosophical Transactions of the Royal Society of London, v. 136, p. 41-62, 1846.

FARADAY, M. Experimental Researches in Electricity. Thirtieth Series. Philosophical Transactions of the Royal Society of London, v. 146, p. 159-180, 1856.

FERNÁNDEZ, M. S.-B.; CALDERÓN, J. M. A.; DÍEZ, P. M. B. Implementation in MATLAB of the adaptive Monte Carlo method for the evaluation of measurement uncertainties. Accreditation and Quality Assurance, v. 14, n. 2, p. 95-106, 19 fev. 2009.

FERREIRA, L. D. R. et al. A heat transfer study aiming optimization of magnetic heat exchangers of thermomagnetic motors. International Journal of Refrigeration, v. 37, n. 1, p. 209-214, 2014.

FERREIRA, L. D. R. et al. Magnetic plates compacted and epoxy bonded. Danish Days on Caloric Materials and Devices. Anais...Roskilde: Technical University of Denmark, 2017. Disponível em: <http://www.danishdays.dk/-/media/Institutter/Energikonvertering/DanishDays/Book_of_abstracts_e-book_standard.ashx?la=da>

GAMA, S. et al. A general approach to first order phase transitions and the anomalous behavior of coexisting phases in the magnetic case. Advanced Functional Materials, v. 19, n. 6, p. 942-949, 2009. 
GILBERT, W. De Magnete - On the loadstone and magnetic bodies and on the great magnet the earth. (1600). Traducao Paul Fleury Mottelay. [s.l: s.n.].

GORE, G. On a momentary molecular change in iron wire. Proc. R. Soc. Lond., v. 17, p. 256-260, 1869.

HALBACH, K. Design of permanent multipole magnets with oriented rare earth cobalt material. Nuclear Instruments and Methods, v. 169, n. 1, p. 1-10, fev. 1980.

HERING, C. Pyro-magnetic motors and generators of electricity. Journal of the Franklin Institute, v. 124, n. 4, p. 278-288, 1887.

HETSRONI, G. STEAM GENERATORS, NUCLEAR. Disponível em: <http://www.thermopedia.com/content/1149/>. Acesso em: 5 maio. 2018.

HOUSTON, E. J.; THOMSON, E. A Curious Thermo-Magnetic Motor. The Journal of the Franklin Institute, v. 107, n. 77, p. 39-40, 1879.

HSU, C. J. et al. Thermomagnetic conversion efficiencies for ferromagnetic materials. Journal of Applied Physics, v. 110, n. 12, 2011.

JOINT COMMITTEE FOR GUIDES IN METROLOGY. Evaluation of measurement data - Guide to the expression of uncertainty in measurement. Guide, v. JCGM 100:2, n. September, 2008a.

JOINT COMMITTEE FOR GUIDES IN METROLOGY. Evaluation of measurement data - Supplement 1 to the "Guide to the expression of uncertainty in measurement" Propagation of distributions using a Monte Carlo method. GUIDE, v. JCGM 101:2, p. 90, 2008b.

KARLE, A. The thermomagnetic Curie-motor for the conversion of heat into mechanical energy. International Journal of Thermal Sciences, v. 40, n. 9, p. 834-842, 2001.

KASAP, S. O. Principles of electronic materials and devices. 3rd. ed. New York, NY: McGraw-Hill, 2006.

KIROL, L. D.; MILLS, J. I. Numerical analysis of thermomagnetic generators. Journal of Applied Physics, v. 56, n. 3, p. 824-828, 1984.

KITANOVSKI, A. et al. Magnetocaloric Energy Conversion. Cham: Springer International Publishing, 2015.

KITANOVSKI, A.; EGOLF, P. W. Thermodynamics of magnetic refrigeration. International Journal of Refrigeration, v. 29, n. 1, p. 3-21, 2006.

KLOTZ, I. NASA Seeks Nuclear Power for Mars. Disponível em: <https://www.scientificamerican.com/article/nasa-seeks-nuclear-power-for-mars/>. Acesso em: 28 jun. 2016.

KNUTAS, A. et al. Cloud-based bibliometric analysis service for systematic mapping studies. Proceedings of the 16th International Conference on Computer Systems and Technologies - CompSysTech '15. Anais...New York, New York, USA: ACM Press, 2015. Disponível em: <http://dl.acm.org/citation.cfm?doid=2812428.2812442>

KONGTRAGOOL, B.; WONGWISES, S. A review of solar-powered Stirling engines and low temperature differential Stirling engines. Renewable and Sustainable Energy Reviews, 
v. 7, n. 2, p. 131-154, 2003.

KOTAS, T. J. The Exergy Method of Thermal Plant Analysis. Glasgow: ButterworthHeinemann Ltd, 1985.

KUHN, T. S. Historical Structure of Scientific Discovery. Science, v. 136, n. 3518, p. 760764, 1 jun. 1962.

LAIRD TECHNOLOGIES. Ceramic Plate Series CP10-71-06: Thermoelectric Modules. Disponível em: <https://assets.lairdtech.com/home/brandworld/files/Laird-ETS-CP-SeriesCP10-71-06-Data-Sheet.pdf>. Acesso em: 12 fev. 2018.

LEE, J. et al. Topology optimization for design of segmented permanent magnet arrays with ferromagnetic materials. Journal of Magnetism and Magnetic Materials, v. 449, p. 571$581,2018$.

LUCIBELLA, M. March 1880: The Curie Brothers Discover Piezoelectricity. APS News, v. 23, n. 3, mar. 2014.

MARTIN, T. C. The Inventions Researches and Writings of Nikola Tesla. New York: The Electrical Engineer, 1894.

MAXIM INTEGRATED. MAX31856 - Precision Thermocouple to Digital Converter with Linearization, 2015. Disponível em: <https://datasheets.maximintegrated.com/en/ds/MAX31856.pdf>. Acesso em: 12 fev. 2018

MCGEE, C. K. A novel magnetic engine. Science, v. III, p. 274-275, mar. 1884.

MERKL, G. G. Step-by-step Thermo-magnetic Motor USA, 1969. Disponível em: <http://www.google.com.br/patents/US3445740>

MINISTÉRIO DE MINAS E ENERGIA. Resenha Energética Brasileira. Brasília: [s.n.]. Disponível em: <http://www.mme.gov.br/documents/10584/3580498/02++Resenha+Energética+Brasileira+2017+-+ano+ref.+2016+\%28PDF\%29/13d8d958-de504691-96e3-3ccf53f8e1e4?version=1.0>. Acesso em: 2 maio. 2018.

MONTEIRO, J. C. B. et al. Determination of the magnetocaloric entropy change by field sweep using a heat flux setup. Applied Physics Letters, v. 105, n. 7, p. 074104, 18 ago. 2014.

MURAKAMI, K.; NEMOTO, M. Some experiments and considerations on the behavior of thermomagnetic motors. IEEE Transactions on Magnetics, v. 8, n. 3, p. 387-389, set. 1972.

NETZSCH - GERÄTEBAU GMBH. Light Flash Apparatus - LFA 467 HyperFlash Series, 2013. Disponível em: <https://www.netzsch-thermal-analysis.com/media/thermalanalysis/brochures/LFA_467_HyperFlash_en_web.pdf>

NOBEL MEDIA. Pierre Curie - Biographical. Disponível em: <https://www.nobelprize.org/nobel_prizes/physics/laureates/1903/pierre-curie-bio.html>. Acesso em: 10 fev. 2018.

OLIVEIRA JUNIOR, S. Exergy. London: Springer London, 2013.

ØRSTED, H. C. Nouvelles expériences de M. Seebeck sur les actions électro-magnétiques. Annales de Chimie et de Physique, v. 22, p. 199-201, 1823. 
OVERSHOTT, K. J. Magnetism: it is permanent. IEE Proceedings-A, v. 138, p. 22-30, 1991.

PALMY, C. A thermo-magnetic wheel. Europhysics News, v. 38, n. 3, p. 32-34, 31 maio 2007.

PECHARSKY, V. K.; GSCHNEIDNER, K. A. Structure, magnetism, and thermodynamics of the novel rare earth-based R5T4 intermetallics. Pure and Applied Chemistry, v. 79, n. 8, p. 1383-1402, 2007.

PFEIFFER VACUUM. PKR 251 - Compact FullRange ${ }^{\text {TM }}$ Gauge, 2005. Disponível em: $<$ http://instrumentation.obs.carnegiescience.edu/FourStar/Documents/FourStar Commercial Manuals/Pfieffer Vacuum/Pfeiffer_Manual.pdf>. Acesso em: 12 fev. 2018

PLACKOWSKI, T.; WANG, Y.; JUNOD, A. Specific heat and magnetocaloric effect measurements using commercial heat-flow sensors. Review of Scientific Instruments, v. 73, n. 7, p. 2755-2765, jul. 2002.

PULKO, B. et al. Epoxy-bonded $\mathrm{La}-\mathrm{Fe}-\mathrm{Co}-\mathrm{Si}$ magnetocaloric plates. Journal of Magnetism and Magnetic Materials, v. 375, p. 65-73, fev. 2015.

RAM, N. R. et al. Review on Magnetocaloric Effect and Materials. Journal of Superconductivity and Novel Magnetism, 3 abr. 2018.

RISSER, M. et al. Construction of consistent magnetocaloric materials data for modelling magnetic refrigerators. International Journal of Refrigeration, v. 35, n. 2, p. 459-467, 2012.

RISSER, M. et al. Improvement and application of a numerical model for optimizing the design of magnetic refrigerators. International Journal of Refrigeration, v. 36, n. 3, p. 950-957, 2013.

ROSA, A. V. DA. Fundamentals of renewable energy processes. $2^{\circ}$ ed. [s.1.] Academic Press, 2012.

SADIN, S. R.; POVINELLI, F. P.; ROSEN, R. The NASA technology push towards future space mission systems. Acta Astronautica, v. 20, n. C, p. 73-77, 1989.

SANCHEZ, R. Technology readiness assessment guide. US Dept. Energy. Washington: [s.n.]. Disponível em: <https://www.directives.doe.gov/directives-documents/400series/0413.3-EGuide-04a/@ @images/file>. Acesso em: 10 maio. 2018.

SCIENTIFIC AMERICAN. The Menges Thermo-Magnetic Generator and Motor. Scientific American, v. 25, n. 633supp, p. 10113-10114, 18 fev. 1888.

SEEBECK, T. J. Magnetische Polarisation der Metalle und Erzedurch Temperatur-Differenz. Abhand Deut. Akad. Wiss. Berlin, v. 7, p. 265-373, 1822.

SMITH, A. et al. Materials challenges for high performance magnetocaloric refrigeration devices. Advanced Energy Materials, v. 2, n. 11, p. 1288-1318, 2012.

SMITH, A. Who discovered the magnetocaloric effect? The European Physical Journal H, v. 38, n. 4, p. 507-517, 3 set. 2013.

SOLOMON, D. Improving the performance of a thermomagnetic generator by cycling the magnetic field. Journal of Applied Physics, v. 63, n. 3, p. 915-921, 1988. 
SOLOMON, D. Design of a thermomagnetic generator. Energy Conversion and Management, v. 31, n. 2, p. 157-173, 1991.

STRAUB, A. P. et al. Harvesting low-grade heat energy using thermo-osmotic vapour transport through nanoporous membranes. Nature Energy, v. 1, n. 7, 2016.

TA INSTRUMENTS. TA - Thermal Conductivity Instruments, 2015. Disponível em: <http://www.tainstruments.com/wp-content/uploads/BROCH-LC-2015-EN.pdf>

TAKAHASHI, Y.; MATSUZAWA, T.; NISHIKAWA, M. Fundamental performance of the disc-type thermomagnetic engine. Electrical Engineering in Japan, v. 148, n. 4, p. 26-33, set. 2004.

TAKAHASHI, Y.; YAMAMOTO, K.; NISHIKAWA, M. Fundamental performance of triple magnetic circuit type cylindrical thermomagnetic engine. Electrical Engineering in Japan, v. 154, n. 4, p. 68-74, mar. 2006.

TAURUS INSTRUMENTS. TCA 300 - Thermal Conductivity Measuring Device. Disponível em: <http://www.taurus-instruments.de/en/thermal-conductivity-measuringdevices/with-heat-flow-meter/tca-300>. Acesso em: 20 nov. 2017.

TCHANCHE, B. F. et al. Low-grade heat conversion into power using organic Rankine cycles - A review of various applications. Renewable and Sustainable Energy Reviews, v. 15, n. 8, p. 3963-3979, 2011.

TESLA, N. Thermomagnetic Motor United StatesGoogle Patents, , 1889. Disponível em: <http://www.google.com.br/patents/US396121>

TESLA, N. Pyromagneto-electric generator US Patent 428,057United States, 1890. Disponível em:

<http://www.google.com/patents?hl=en\&lr=\&vid=USPAT428057\&id=6CpOAAAAEBAJ \& oi=fnd\&dq=Pyromagneto-Electric+Generator\&printsec $=$ abstract $>$

TEXAS INSTRUMENTS. ADS111x Ultra-Small, Low-Power, $I^{2} \mathrm{C}$-Compatible, 860-SPS, 16-Bit ADCs: With Internal Reference, Oscillator, and Programmable Comparator, 2009. Disponível em: 〈http://www.ti.com/lit/ds/symlink/ads1114.pdf>. Acesso em: 12 fev. 2018

THERMONAMIC MODULE. Specification of Thermoelectric Module - TEC1-12706, 2017. Disponível em: 〈http://www.thermonamic.com/tec1-12706-english.pdf〉. Acesso em: 12 fev. 2018

TOULOUKIAN, Y. S. et al. Thermal Conductivity: Metallic Elements and Alloys (Thermophysical Properties of Matter Vol. 1). Boston, MA: Springer US, 1970a.

TOULOUKIAN, Y. S. et al. Thermal Conductivity: Nonmetallic Solids (Thermophysical Properties of Matter Vol. 2). New York, NY: Plenum Publishing Corporation, 1970b.

TOUlOUKIAN, Y. S.; LILEY, P. E.; SAXENA, S. C. Thermal Conductivity: Nonmetallic Liquids and Gases (Thermophysical Properties of Matter Vol. 3). New York, NY: Plenum Publishing Corporation, 1970.

TRITT, T. M.; WESTON, D. Measurement Techniques and Considerations for Determining Thermal Conductivity of Bulk Materials. In: TRITT, T. M. (Ed.). . Thermal Conductivity: 
theory, properties and applications. New York: Kluwer Academic / Plenum Publishers, 2005. p. 187-203.

UJIHARA, M.; CARMAN, G. P.; LEE, D. G. Thermal energy harvesting device using ferromagnetic materials. Applied Physics Letters, v. 093508, n. 2007, p. 1-4, 2007.

UNITED NATIONS. RES/70/1. Transforming our world: the 2030 agenda for sustainable development. Disponível em:

<http://www.un.org/ga/search/view_doc.asp?symbol=A/RES/70/1\&Lang=E>. Acesso em: 2 maio. 2018.

VUARNOZ, D. et al. Quantitative feasibility study of magnetocaloric energy conversion utilizing industrial waste heat. Applied Energy, v. 100, p. 229-237, 2012.

WAHL, W. H. Electricity Directly from Fuel. The Manufacturer and Builder, v. XIX, p. 194-195, set. 1887.

WEISE, B. et al. Anisotropic thermal conductivity in epoxy-bonded magnetocaloric composites. Journal of Applied Physics, v. 120, n. 12, 2016.

WEISS, P.; PICCARD, A. Le phénomène magnétocalorique. Journal de Physique Théorique et Appliquée, v. 7, n. 1, p. 103-109, 1917.

WIPO. International Patent Classification. Disponível em: <http://www.wipo.int/classifications/ipc/en/>. Acesso em: 11 jun. 2018.

WOOLNOUGH, D. WASTE HEAT RECOVERY. Disponível em: <http://www.thermopedia.com/content/1250/>. Acesso em: 8 maio. 2018.

YANG, J. Theory of Thermal Conductivity. In: TRITT, T. M. (Ed.). . Thermal Conductivity: theory, properties and applications. New York, NY: Kluwer Academic / Plenum Publishers, 2005. p. 1-20.

ZHU, F. et al. Enhanced mechanical properties and large magnetocaloric effect in epoxybonded Mn0.98CoGe. Scripta Materialia, v. 150, p. 96-110, 2018. 